\title{
Identification of Anti-tubercular Compounds in Marine Organisms from Aotearoa
}

by

Nathaniel Dasyam

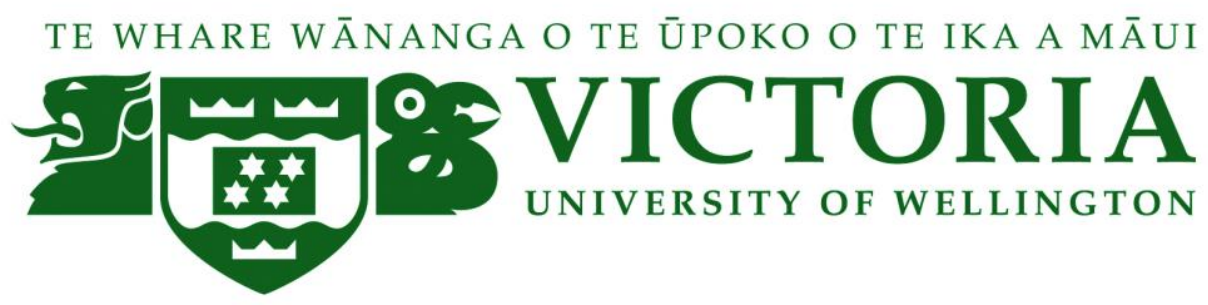

\begin{abstract}
A thesis
submitted to Victoria University of Wellington

in fulfilment of the requirements for the degree of

Doctor of Philosophy in Molecular Microbiology
\end{abstract}

2014 


\section{Supervisors}

Dr. Robert Keyzers

Senior Lecturer, School of Chemistry

Victoria University of Wellington

Wellington, New Zealand

Dr. Ronan Francis O’Toole

Assistant Professor, Department of Clinical Microbiology

Trinity College Dublin

Ireland 


\section{Abstract}

Tuberculosis (TB) is responsible for more than one million human deaths per year globally, more than any other disease caused by a bacterial pathogen. Although effective treatments exist, the long duration of the current treatment regimen as well as associated drug toxicities can lead to patient non-compliance. This has resulted in the spread of drug resistance which is highlighted by the isolation of extensively drug-resistant strains of Mycobacterium tuberculosis complex in all regions of the globe. The management of drug-resistant TB cases requires an extended treatment duration of 18 months minimum post sputum-culture conversion, using drugs that are more frequently linked to adverse side-effects. Therefore, there is a need to identify new drugs that shorten treatment time, and also exhibit better tolerance in patients.

This thesis describes the screening of crude marine extract libraries for the identification of novel anti-tubercular compounds. The libraries consisted of extracts from New Zealand marine organisms that were screened for growth inhibitory activity using a surrogate TB model organism $M$. smegmatis, and subsequently were validated against $M$. tuberculosis. Bioassay-guided fractionation led to the identification of two new compounds as well as a mixture of known compounds. In addition to anti-mycobacterial activity, the spectrum of activity of the isolated compounds was also investigated.

Fractionation of the liver extract of the blobfish Psychrolutes marcidus led to the identification of a mixture of four common fatty acids as the major bioactive components. Testing each of the fatty acids individually identified palmitic acid as the most active component against M. tuberculosis H37Ra. This is at odds with current understanding of the fatty acid parameters required for bioactivity in terms of chain length and degree of unsaturation.

Bioassay-guided fractionation of a Latrunculia sp. sponge extract led to the isolation of a novel taurinated terpene rimarikiamide A (98). This compound was initially believed to be responsible for the observed anti-mycobacterial activity, however, further purification led to partitioning of the anti-mycobacterial activity away from 98. The taurinated terpene rimarikiamide A was nevertheless tested for activity in 
mammalian cell lines and was found to exhibit higher levels of growth inhibition towards HL-60 cells with respect to HEK cells.

Working with Xiphophora chondrophylla, a New Zealand brown alga, led to the identification of the amino alcohol 3-epi-xestoaminol C (156) a stereoisomer of a known compound, as the active principle. This compound displayed the same level of activity in all microorganisms tested, however it proved to be more active against HL-60 cells. To further investigate the mode of action of $\mathbf{1 5 6}$, chemical genetics was employed to indicate the relevant genetic networks that the compound interacts with. This led to the identification of expected pathways such as lipid metabolism as well as cytoskeletal organization, which have been linked to this class of compounds. Furthermore this work also identified new processes such as RNA catabolism, protein targeting and phosphorylation in addition to several unknown processes.<smiles>CC(C)=CCC/C(C)=C/CC(O)/C(C)=C/CCC(C)CC(=O)NCCS(=O)(=O)O</smiles>

98<smiles>CCCCCCCCCCC[C@H](C)N</smiles> 


\section{Acknowledgements}

I would like to thank Assoc. Prof. Ronan F. O'Toole, who made it possible for me to undertake this project. Thank you for your guidance in this project, and for the opportunity to do my $\mathrm{PhD}$. I gratefully acknowledge Dr. Robert Keyzers for guiding a simple biologist through the field of natural products chemistry, especially through its many twists and turns. For all the knowledge you imparted to me I am grateful. For the patience in teaching me natural products chemistry and when it came to the finer points of writing I am always in your debt. Without your help this project would not have come to completion.

I am also thankful to Assoc. Prof. Peter T. Northcote for the wealth of advice in NMR and natural products chemistry in addition to allowing me access to the VUW marine extract collection. Dr. Joanne Harvey, thank you for providing advice as well as chemical reagents for derivatisation reactions. Dr. Mikhail Vyssotski of Callaghan Innovation, thank you for running our GC-MS analysis when our instrument was down. Drs. Vicky Webb and Sally Anderson, thank you for providing plates of extracts from the NIWA library. Assoc. Prof. Joe Zuccarello, thank you for your help with marine alga identification.

Drs. Ariane Chan and Arun Kanakkanthara, thank you for teaching the MTT assay to me, and Dr. Laura Green as well as Arun for performing FACS analysis for the G2/M block assay. Nazmi H. Fadzilah thank you for performing the yeast chemical genetic screens and Dr. Andrew B. Munkacsi thank you for helping analyse the data. To all the technical staff of SBS and SCPS I am grateful to you for allowing me the use of equipment.

To the members of the microbiology group Sandi Dempsey, Richard Campen, Ian Bassett, Drs. Shahista Nisa, Mudassar Altaf and Gareth Prosser thank you for all the helpful advice and company. In particular, I would like to thank Dr. Christopher Miller for his advice and guidance in teaching me all that I needed to learn when I first joined the lab. Richard thank you for your advice and guidance in all things biology, especially when performing the transposon mutant library screens. I would also like to thank Dr. Peter Bosch for all his advice. 
To the members of the marine natural products group, Peter Clark, Helen Woolner, Danica Carter, Emma Aitken, Chriselle Braganza, Vidhiya Damodaran and Drs. Jacquie Barber and Ameet Jonathan Singh thankyou for your excellent company, guidance and patience. I would like to particularly acknowledge Jono for all the time spent teaching me how to run the $600 \mathrm{MHz}$ NMR instrument as well as structural elucidation advice and for always guiding me when I was lost in chemistry, I'm glad I have made a good friend like you.

Thank you Emma, Juliane Bock and Barbara Paul for making up the VUW 96-well plate marine extract library. Emma, thanks for helping me through my work on the Latrunculia sponges and for being a good friend and hearing me out when things didn't always go to plan, both in training and in the lab. Chriselle, thank you for being crazy and always cheering the whole lab up, you have been an awesome friend and great company being the only other biologist in the lab. Vidhiya, thank you for your advice in all the tricky parts of chemistry, you have always been there when things did not work out. I will never forget your friendship and constant support you've shown me. After all the music wars, crazy-yet-safe pranks, and our constant 'debates' you are a true friend that I will never forget.

To my friends Romano, Nick, Marie and Tate thanks for your motivation and support. The guys from karate, for helping me stay sane throughout the years, thank you.

Finally I'd like to thank my mother, father and sister for their steadfast support, trying to understand why science doesn't always work perfectly and the never ending question "when will you be done?" Mum thankyou for your love, support and abundance of food you always had ready for me, without your help and support I would not be here. 


\section{Table of Contents}

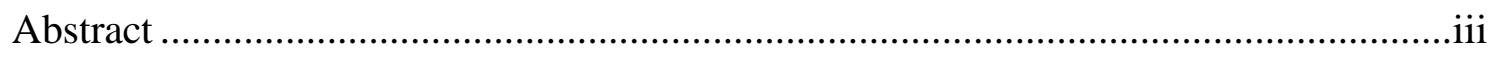

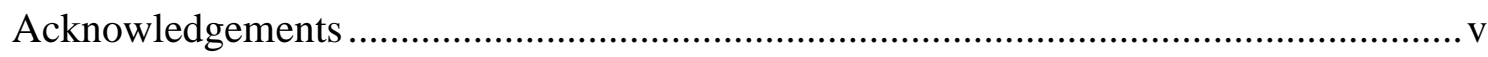

Table of Contents ................................................................................................. vii

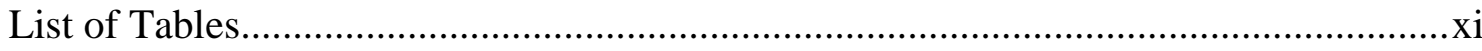

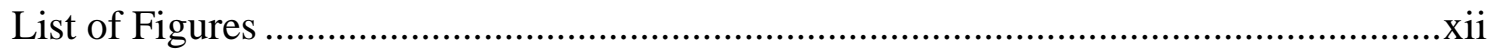

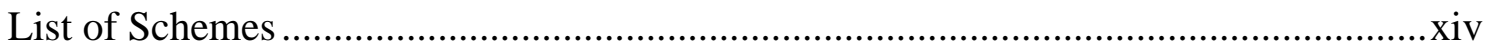

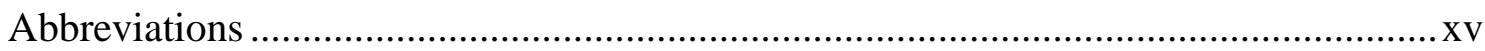

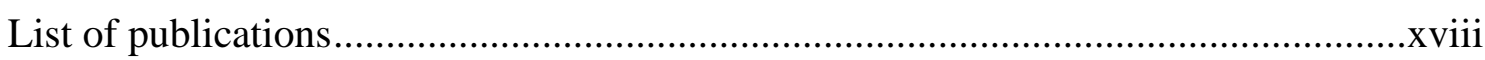

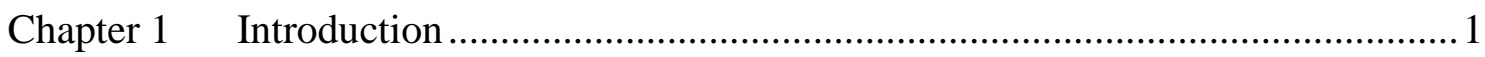

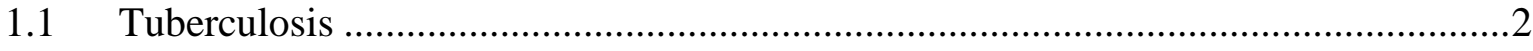

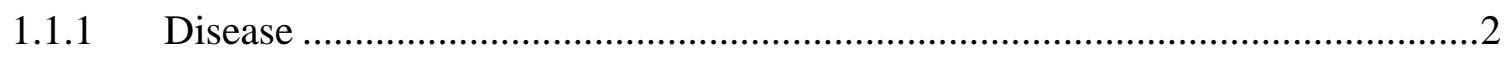

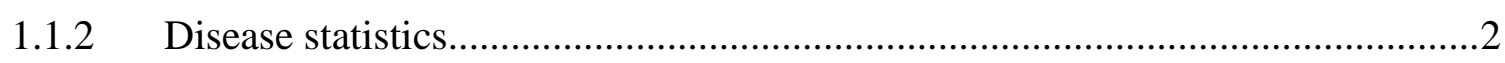

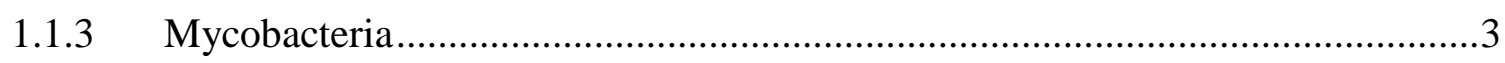

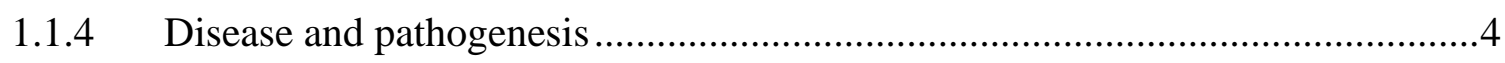

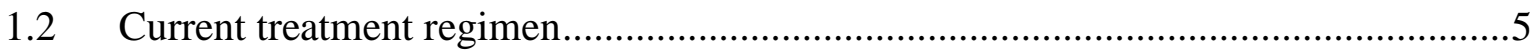

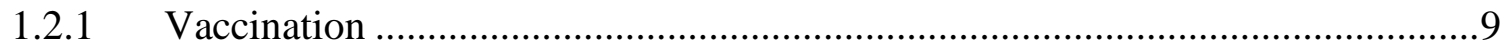

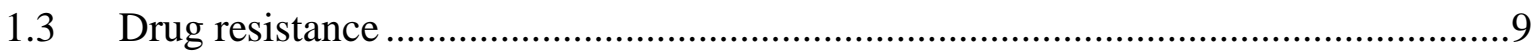

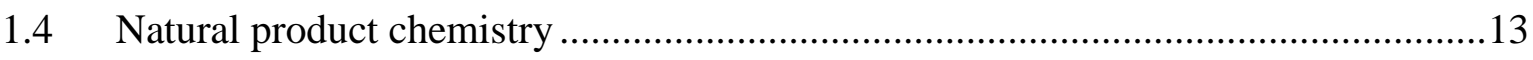

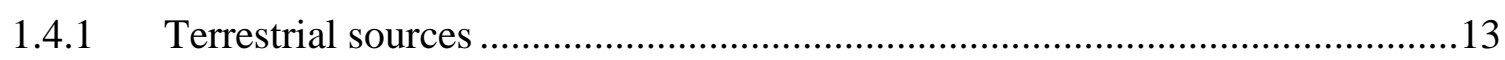

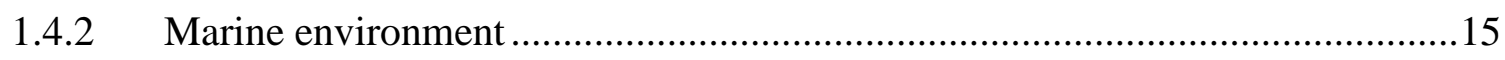

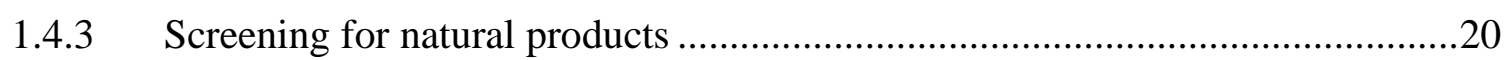

1.4.4 Structure-activity relationships and mechanism of action studies ....................22

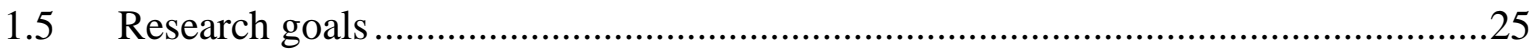

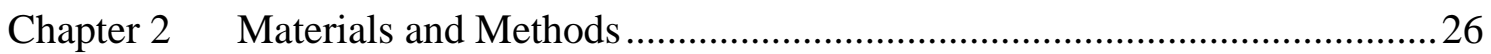

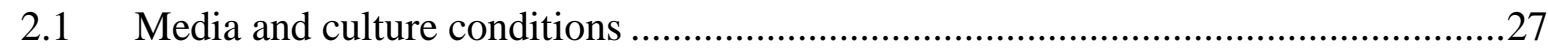

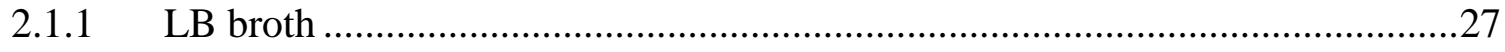

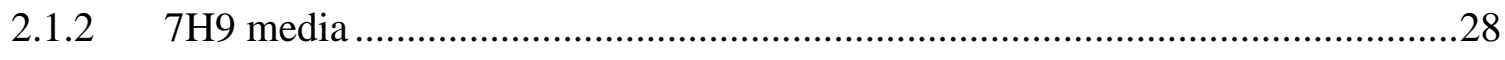

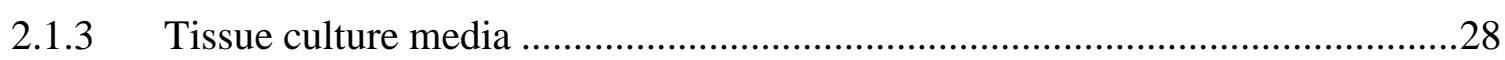

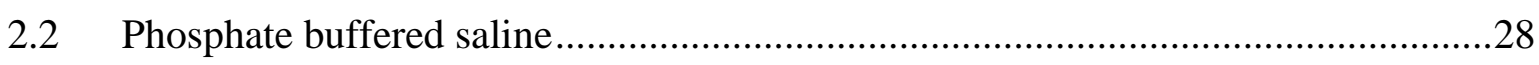

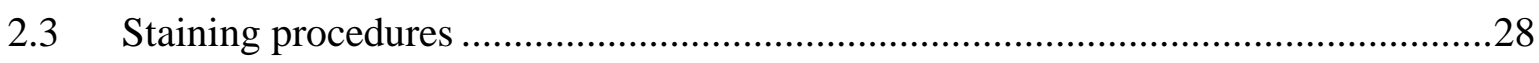

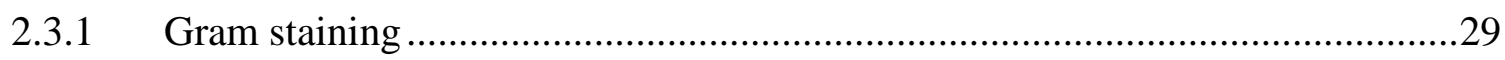

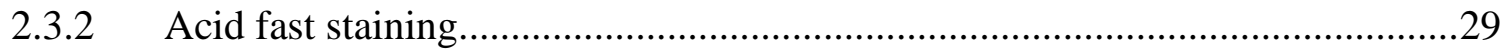

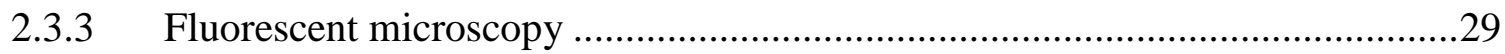


2.4 Transformation of mycobacteria .29

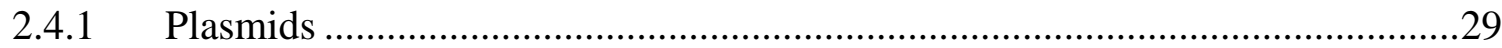

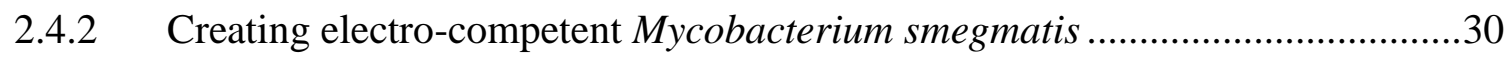

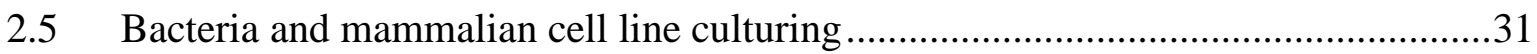

2.5.1 Culturing of Mycobacterium smegmatis .............................................................

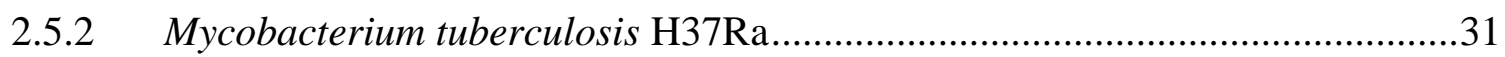

2.5.3 Staphylococcus aureus, Escherichia coli and Pseudomonas aeruginosa...........32

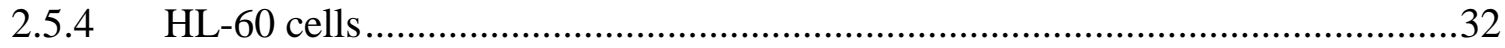

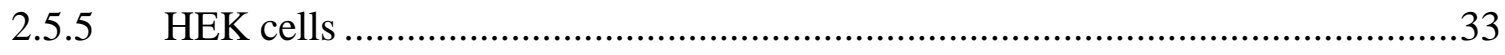

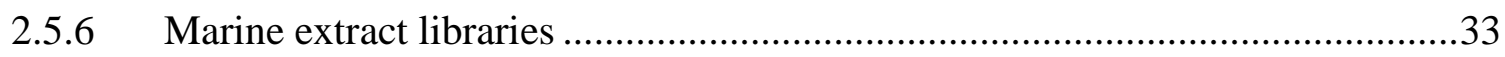

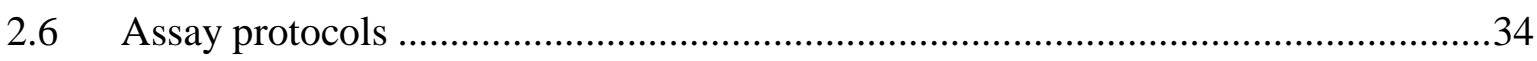

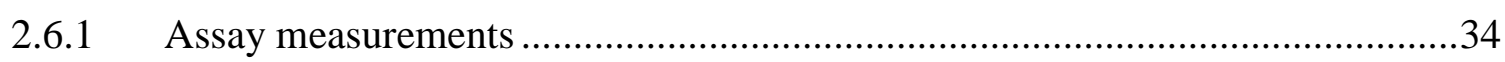

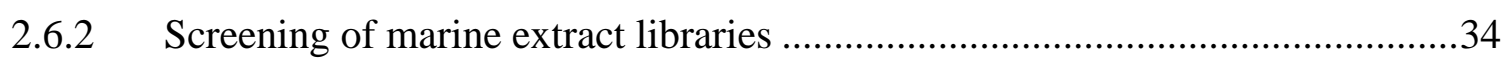

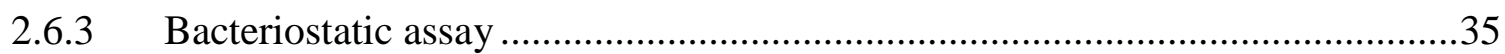

2.6.4 Bacteriostatic assay set up in M. tuberculosis H37Ra .....................................36

2.6.5 Stationary phase assay protocol in M. tuberculosis H37Ra...............................36

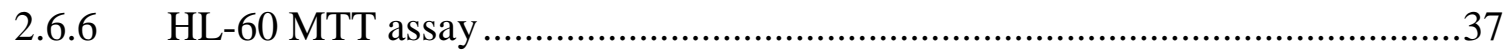

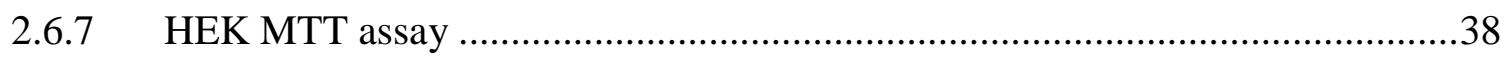

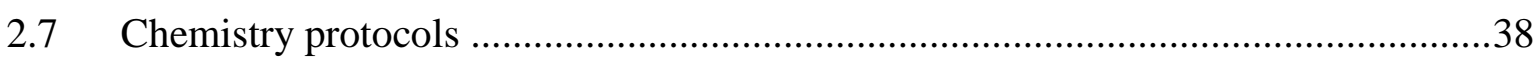

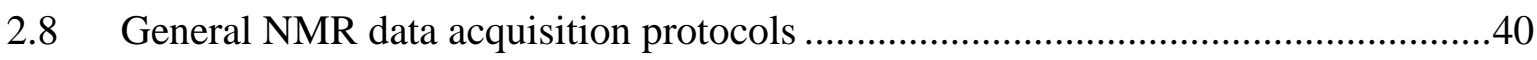

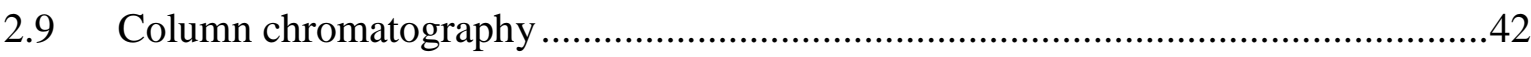

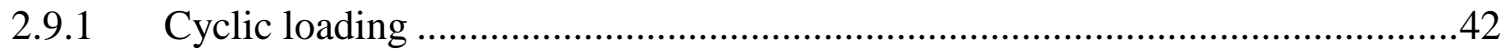

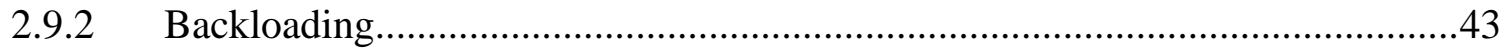

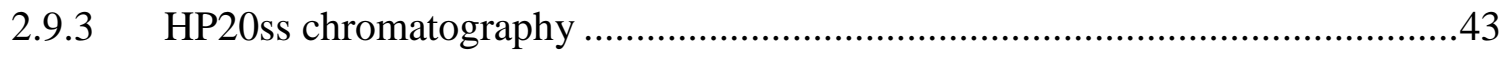

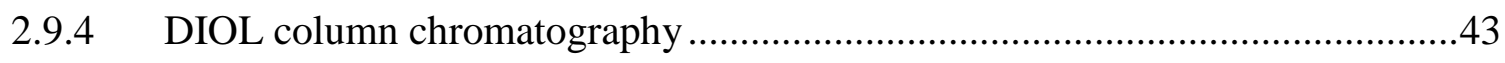

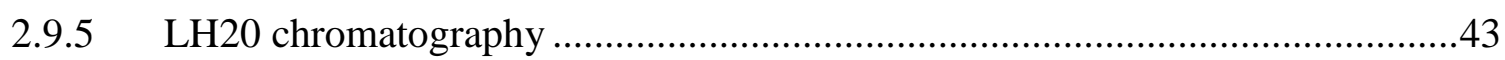

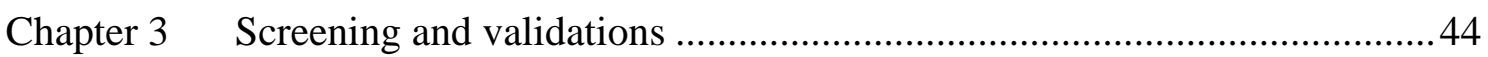

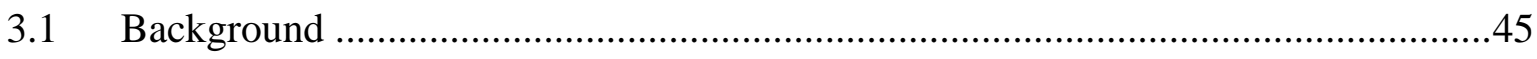

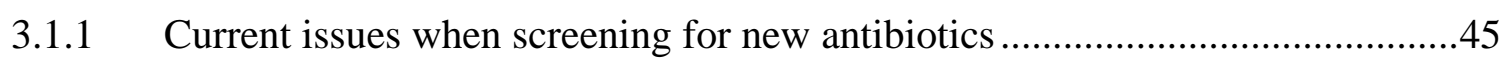

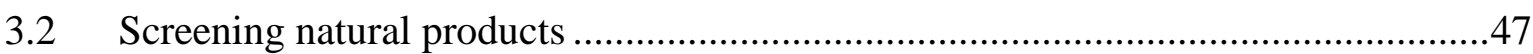

3.2.1 Overcoming the challenges in natural product research ................................48

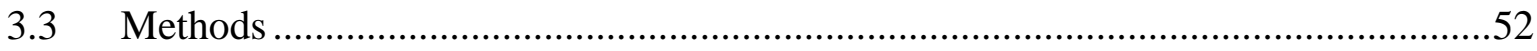

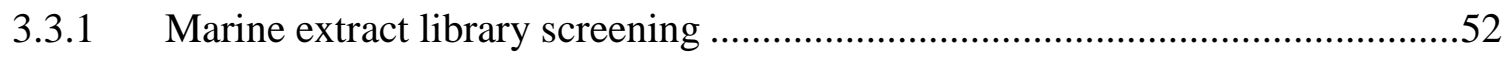

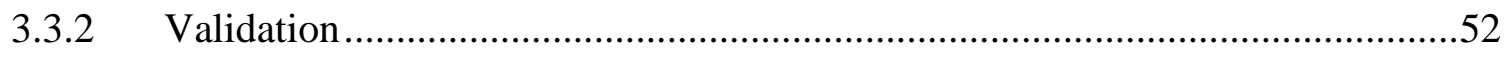

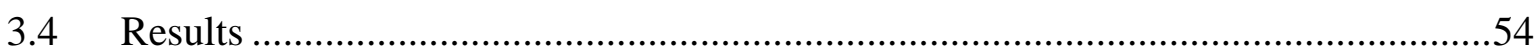

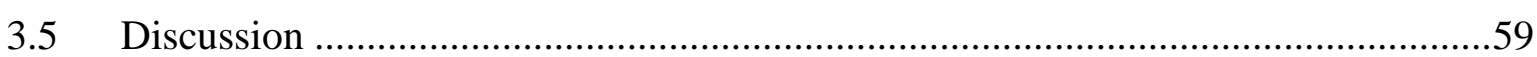

Chapter $4 \quad$ Bioactive fatty acids from Psychrolutes marcidus ...................................62 62 


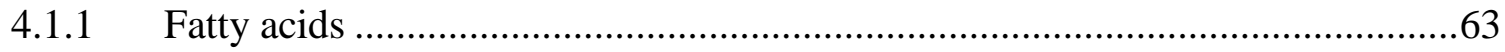

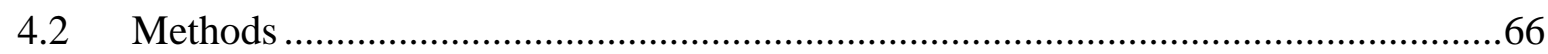

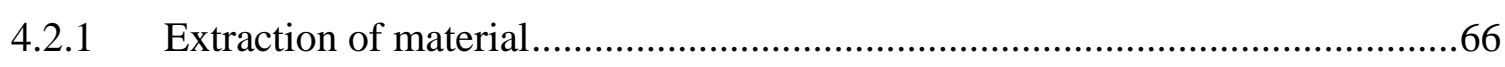

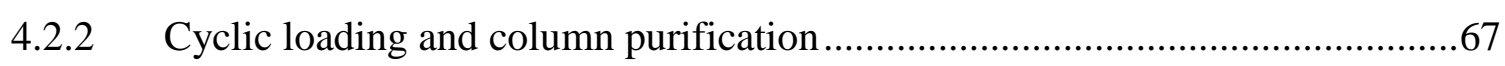

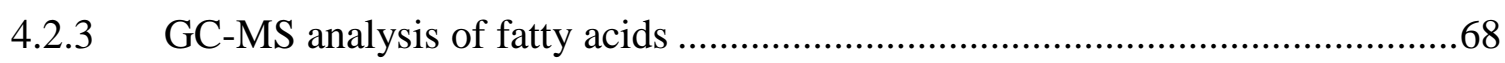

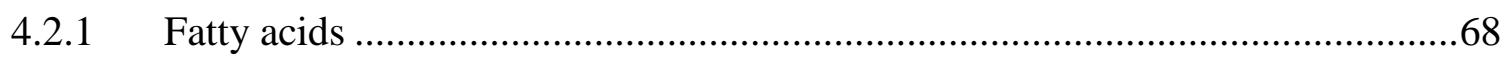

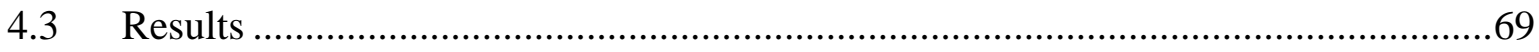

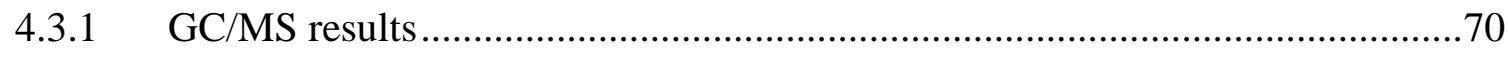

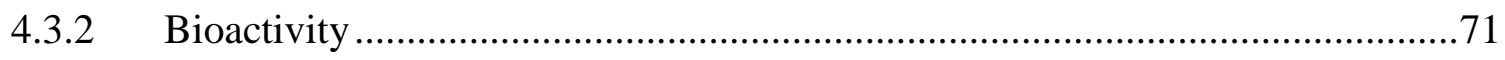

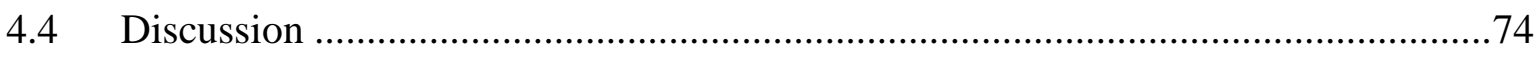

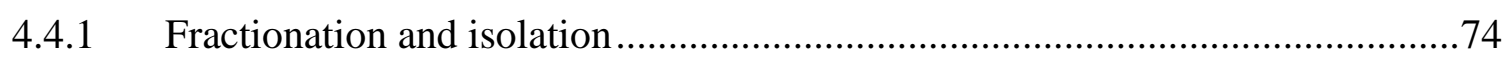

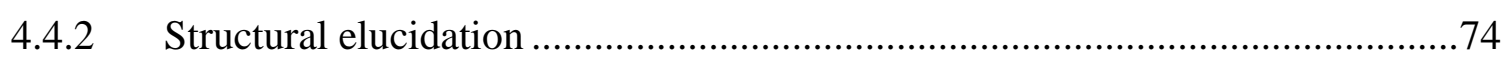

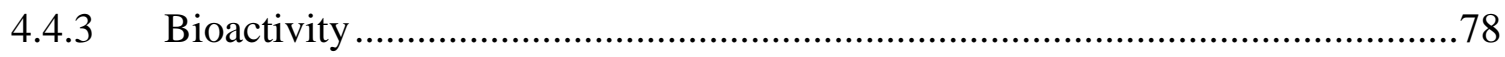

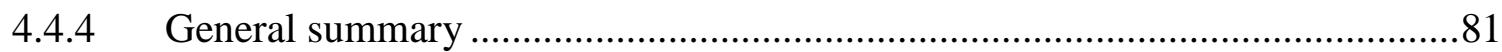

Chapter 5 Rimarikiamide A from Latrunculia sp................................................. 83

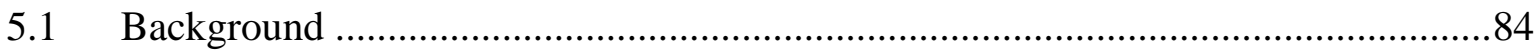

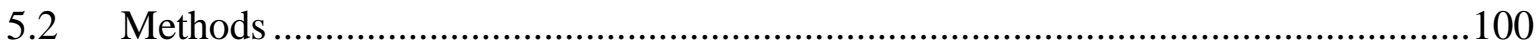

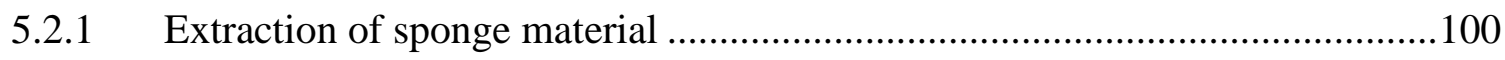

5.2.2 Isolation schemes for rimarikiamide A ….................................................. 101

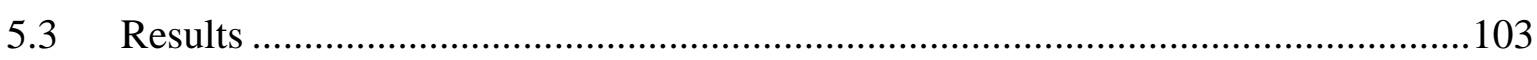

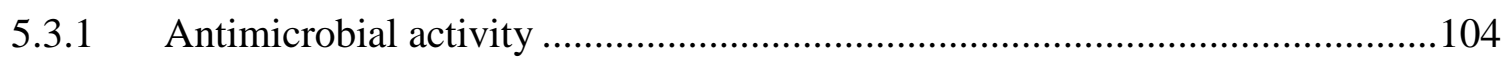

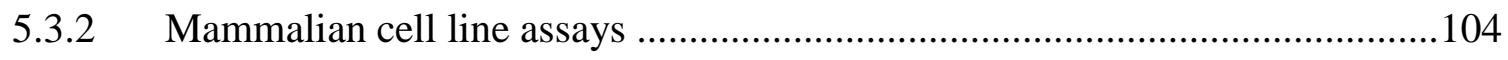

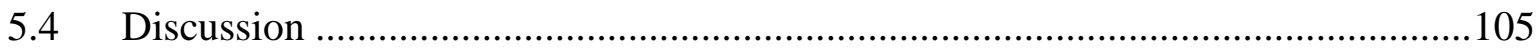

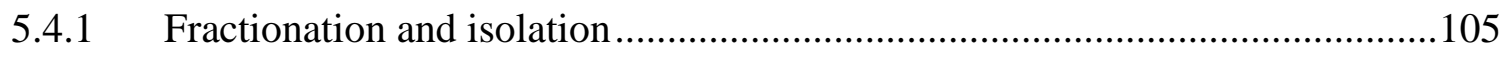

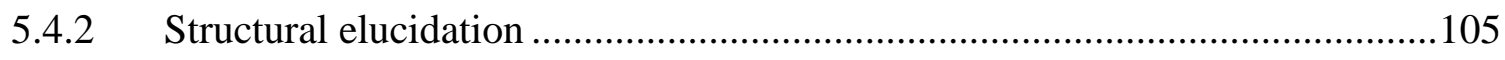

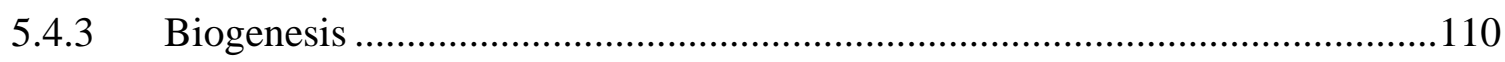

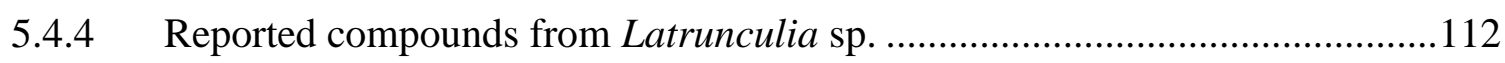

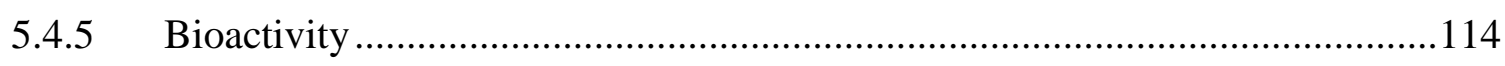

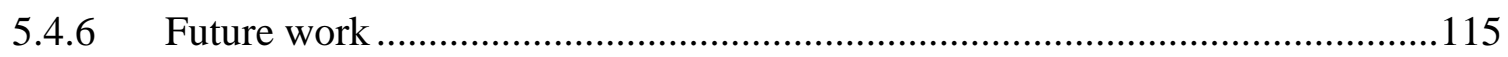

Chapter 6 3-Epi-xestoaminol C from Xiphophora chondrophylla ......................... 116

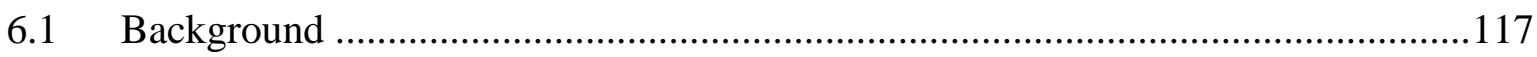

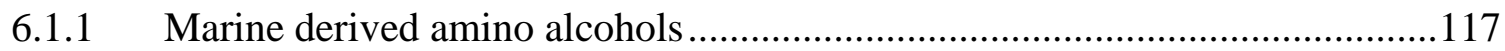

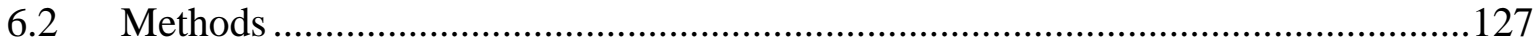

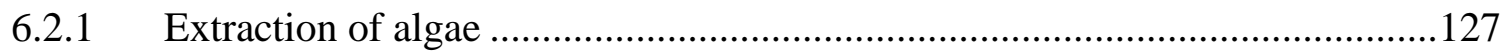

6.2.2 Isolation schemes for 3-epi-xestoaminol C (156) ......................................... 129

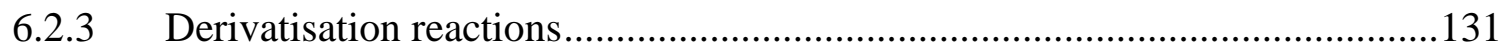




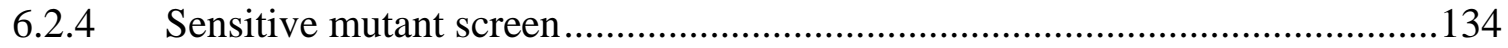

6.2.5 Resistant mutant generation and screen protocol ..........................................135

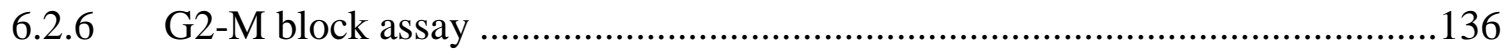

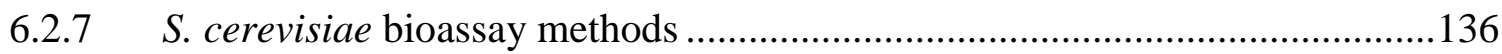

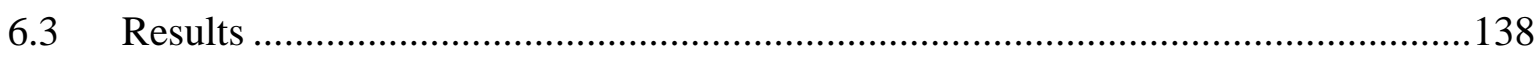

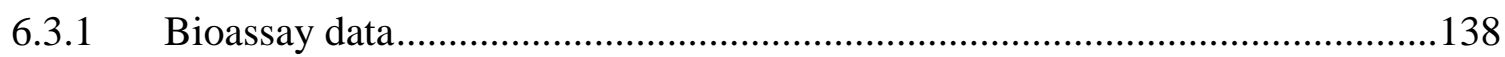

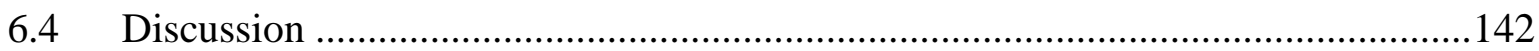

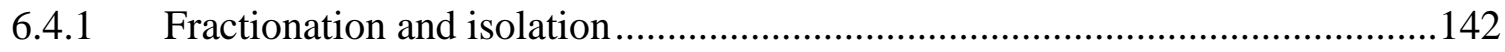

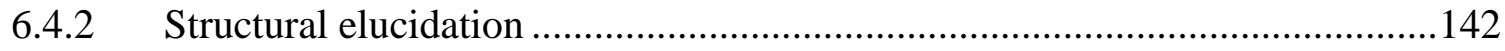

6.4.3 Compounds from the genus Xiphophora …................................................... 151

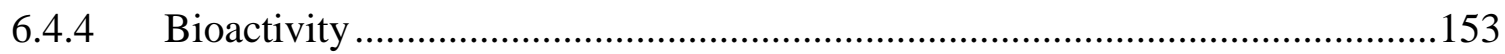

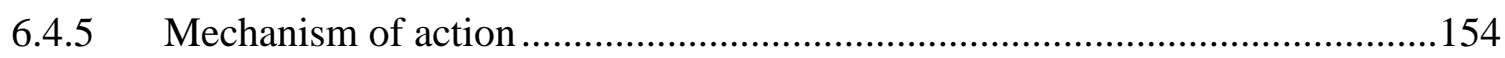

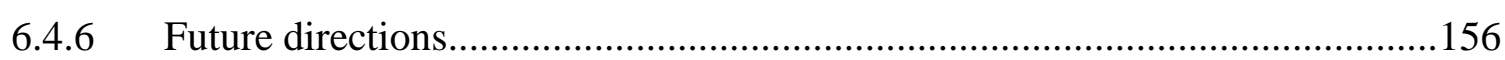

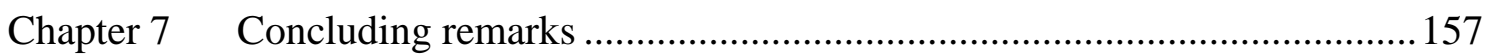

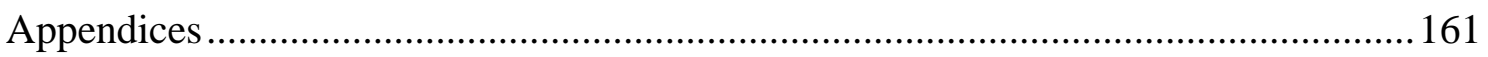

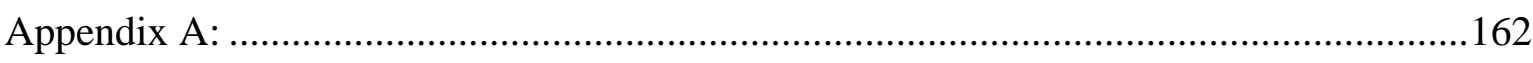

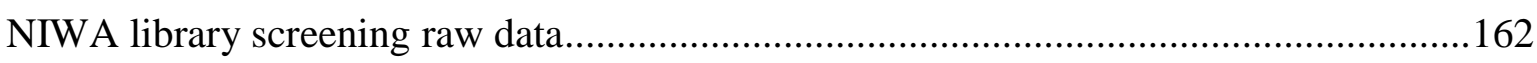

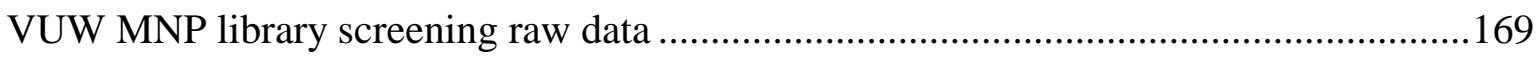

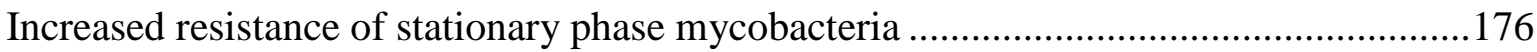

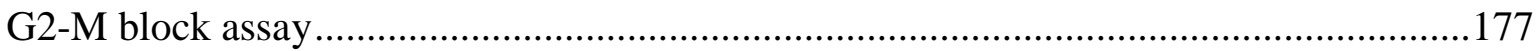

Appendix B: Fatty acid mixture from Psychrolutes marcidus ..........................................178

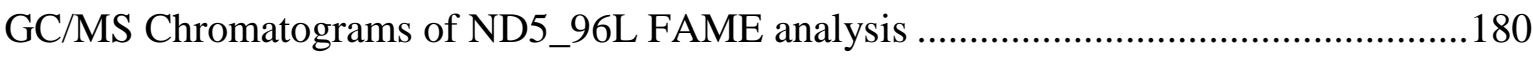

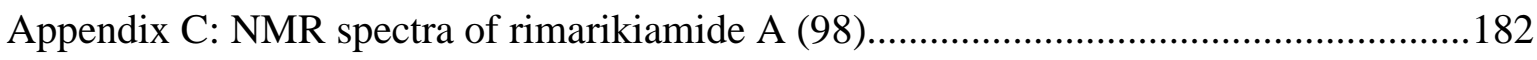

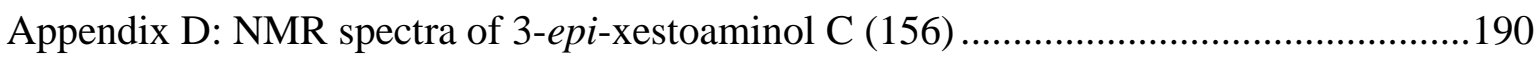

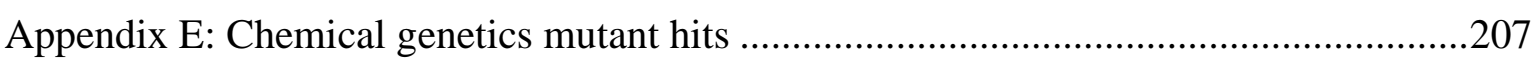

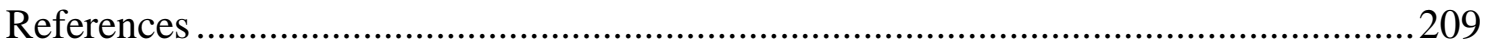




\section{List of Tables}

Table 1.1 Current first and second line anti-tuberculosis drugs .................................

Table 1.2 Common mechanisms of drug resistance to first line anti-TB drugs.............. 10

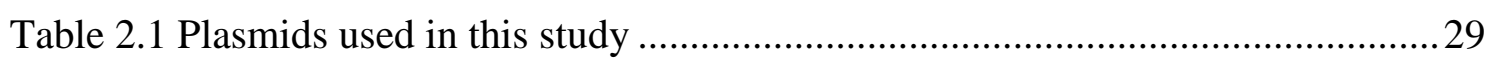

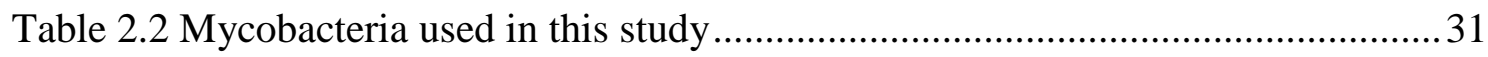

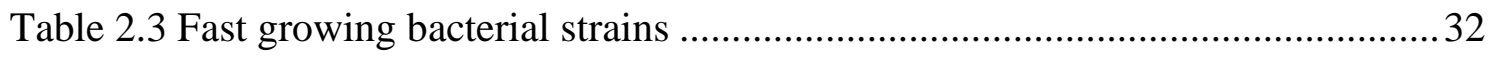

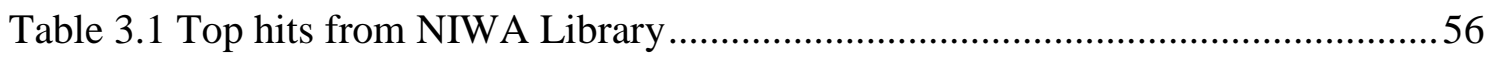

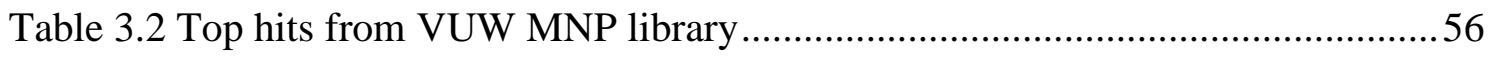

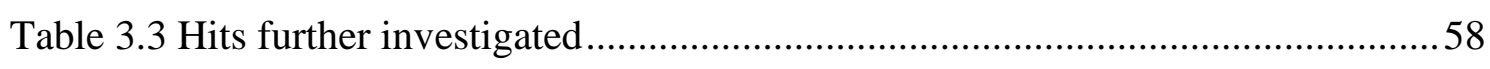

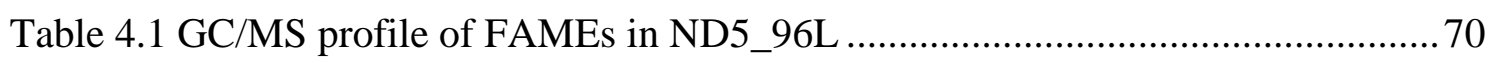

Table 4.1 Broad- spectrum activity of active mixture of fatty acids ............................ 72

Table 4.2 Bioactivity profile of individual fatty acids .............................................. 72

Table 4.3 Comparison of $\mathrm{MIC}_{90}$ values of artificial mixtures against $M$. tuberculosis

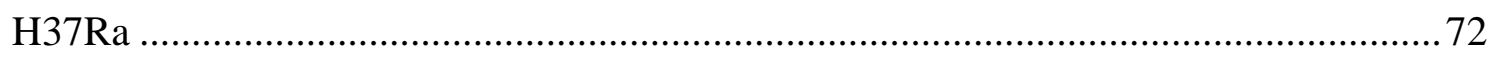

Table 4.4 Bioactivity of fatty acid mixtures vs. M. tuberculosis H37Ra....................... 73

Table $5.1{ }^{1} \mathrm{H}(600 \mathrm{MHz})$ and ${ }^{13} \mathrm{C}(150 \mathrm{MHz}) \mathrm{NMR}$ data $\left(\mathrm{d}_{6}\right.$-DMSO) for rimarikiamide

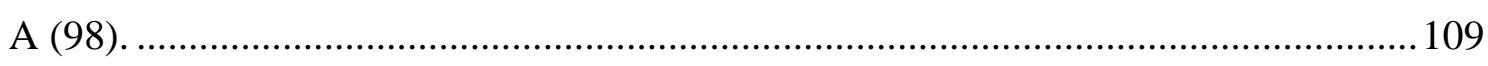

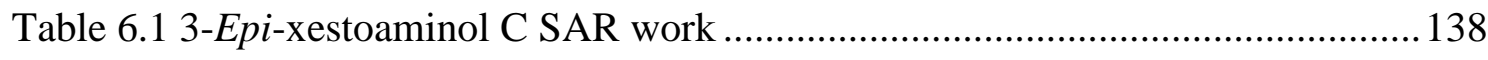

Table $6.2{ }^{1} \mathrm{H}(600 \mathrm{MHz}),{ }^{13} \mathrm{C}(150 \mathrm{MHz}) \mathrm{NMR}$ data of 3-epi-xestoaminol-C (156)

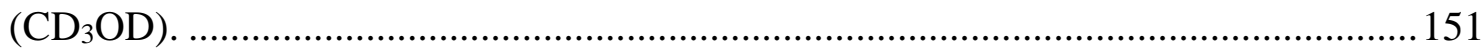

Table 7.1 Raw data of NIWA library screen vs. M. smegmatis ................................... 162

Table 7.2 Raw data of VUW MNP library screen vs. M. smegmatis ........................... 169 


\section{List of Figures}

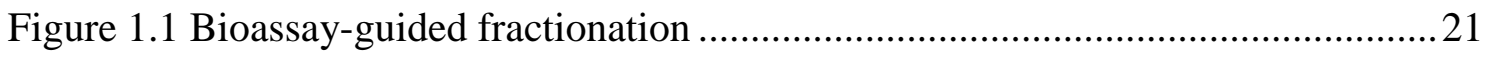

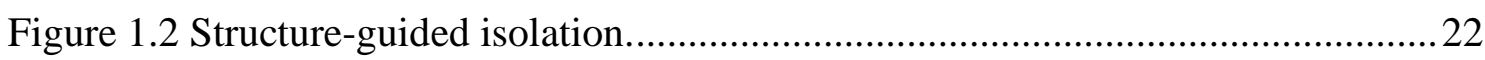

Figure 2.1 General set up of a 96-well plate for screening marine extract libraries ....... 35

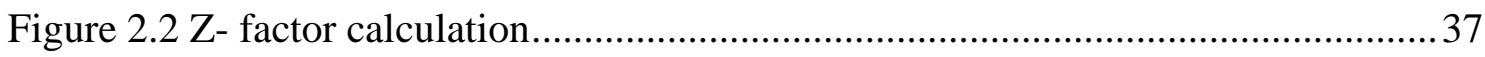

Figure 3.1 M. smegmatis screening results from the NIWA library ..............................54

Figure 3.2 M. smegmatis screening results from the VUW MNP library .......................55

Figure 3.3 Spectrum of activity and overlap of hits from VUW MNP library screen....57

Figure 4.1 ND5_96L vs. stationary phase M. tuberculosis H37Ra .............................. 71

Figure 4.2 ND5_96L vs. mammalian cell lines ......................................................... 71

Figure 4.3 Comparison of ${ }^{13} \mathrm{C} \mathrm{NMR}\left(\mathrm{CDCl}_{3}, 150 \mathrm{MHz}\right)$ spectra of ND5_96L (top), cis-vaccenic (middle) and oleic acids (bottom) at full spectral width.

Figure 4.4 Comparison of ${ }^{13} \mathrm{C} \mathrm{NMR}\left(\mathrm{CDCl}_{3}, 150 \mathrm{MHz}\right)$ spectra of ND5_96L (top), cis-vaccenic (middle) and oleic acids (bottom) at a specific range of interest. .75

Figure 4.5 Comparison of ${ }^{1} \mathrm{H}$ NMR $\left(\mathrm{CDCl}_{3}, 600 \mathrm{MHz}\right)$ spectra of ND5_96L (top), cis-vaccenic (middle) and oleic acids (bottom) at full spectral width. 76

Figure 4.6 Comparison of ${ }^{1} \mathrm{H} \mathrm{NMR}\left(\mathrm{CDCl}_{3}, 600 \mathrm{MHz}\right)$ spectra of ND5_96L (top), cis-vaccenic (middle) and oleic acids (bottom) at a specific range of interest. .76

Figure 4.7 Comparison of ${ }^{1} \mathrm{H}$ NMR $\left(\mathrm{CDCl}_{3}, 600 \mathrm{MHz}\right)$ spectra of ND5_96L (top), cis-vaccenic (middle) and oleic acids (bottom) at a specific range of interest. .77

Figure 5.1 Loss of anti-mycobacterial activity of rimarikiamide A …........................ 104

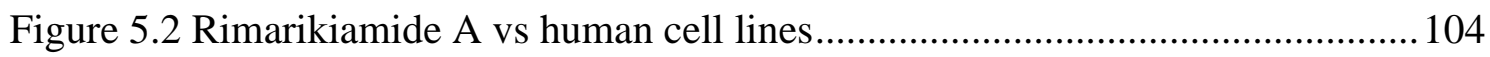

Figure 5.3 Key COSY and HMBC correlations establishing substructure 1 ................106

Figure 5.4 Key COSY and HMBC correlations establishing substructure 2 ................ 106

Figure 5.5 Key COSY and HMBC correlations establishing substructure 3 ............... 107

Figure 5.6 Key COSY and HMBC correlations establishing substructure 4 ............... 107

Figure 5.7 Key COSY and HMBC correlations establishing substructure 5 ............... 107

Figure 5.8 Key COSY and HMBC correlations establishing substructure 6............... 108

Figure 5.9 Key ROESY correlations establishing the alkene geometry of rimarikiamide

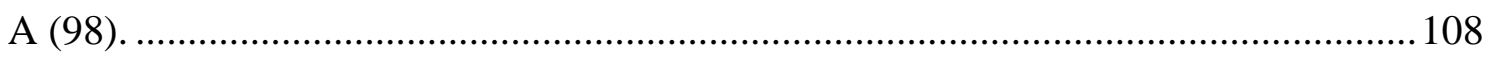

Figure 5.10 Proposed biogenesis of rimarikiamide A (98) ...................................... 111

Figure $5.11{ }^{1} \mathrm{H}$ NMR $\left(\mathrm{CD}_{3} \mathrm{OD}, 600 \mathrm{MHz}\right)$ spectrum spectra of ND4_47D+E............. 114 
Figure 6.1 Antimicrobial activity of 3-epi-xestoaminol C (156).

Figure 6.2 Stationary phase assay 140

Figure 6.3 3-epi-xestoaminol C (156) vs. mammalian cell lines 140

Figure 6.4 Network map of hits from yeast chemical genetics screening of 3-epixestoaminol C (156).

Figure 6.5 Key COSY and HMBC correlations establishing substructure 1 143

Figure 6.6 Key COSY and HMBC correlations establishing substructure 2 143

Figure 6.7 Proposed planar structure 143

Figure 6.8 TOCSY correlations 144

Figure 6.9 NOE correlations of oxazolidinone 157 144

Figure 6.10 Change in multiplicity of the oxymethine ${ }^{1} \mathrm{H}$ NMR resonance for $R$-ester (160, top panel) and $S$-ester (161, bottom panel) in $\mathrm{CDCl}_{3}(600 \mathrm{MHz})$. 146

Figure $6.11 \Delta \delta{ }^{1} \mathrm{H}$ NMR data of MTPA esters 160 and 161 in $\mathrm{CDCl}_{3}(600 \mathrm{MHz}) \ldots . .146$ Figure $6.12{ }^{1} \mathrm{H}$ NMR oxymethine resonance for $R$-ester (160, top panel) and $S$-ester (161, bottom panel) in $\mathrm{CD}_{3} \mathrm{OD}(600 \mathrm{MHz})$.

Figure $6.13 \Delta \delta{ }^{1} \mathrm{H}$ shifts between MTPA esters 160 and 161 of $N$-acetylated xestoaminol $\mathrm{C}$ in $\mathrm{CD}_{3} \mathrm{OD}$ 148

Figure 6.14 Oxy- and aminomethine region, ${ }^{1} \mathrm{H}$ NMR spectra for the $R$-ester (160, top panel) and $S$-ester (161, bottom panel) in $\mathrm{CD}_{3} \mathrm{OD}(600 \mathrm{MHz})$ 148

Figure 6.15 Aliphatic region, ${ }^{1} \mathrm{H}$ NMR spectra for the $R$-ester (160, top panel) and $S$ ester (161, bottom panel) in $\mathrm{CD}_{3} \mathrm{OD}(600 \mathrm{MHz})$.

Figure 6.16 Steric effects influencing ${ }^{19} \mathrm{~F}$ NMR shifts 149

Figure $6.17{ }^{19} \mathrm{~F}$ NMR shifts $(280 \mathrm{MHz})$ in $\mathrm{CD}_{3} \mathrm{OD}$ for the $R$-ester (160, top panel) and $S$-ester (161, bottom panel) in $\mathrm{CD}_{3} \mathrm{OD}$. 150 


\section{List of Schemes}

Scheme 4.1 Isolation scheme of fatty acids from Psychrolutes marcidus 67

Scheme 5.1 Original isolation scheme for rimarikiamide A (98) from Latrunculia sp. 101

Scheme 5.2 Optimised isolation scheme for rimarikiamide A (98) from Latrunculia sp. 102

Scheme 6.1 Original isolation scheme for 3-epi-xestoaminol C (156) from Xiphophora chondrophylla. 129

Scheme 6.2 Optimised isolation scheme for 3-epi-xestoaminol C (156) from Xiphophora chondrophylla. 130 


\section{Abbreviations}

A

AU

br

CDI

$\mathrm{CFU}$

d

DAD

DCM

DMAP

dd

ddd

DIOL

DMF

DMSO

DOTS

EDCI

EGFR

ESBL-E

EtOAc

FACS

FAME

FFA

GC-FID

GFP

$\mathrm{H}$

H37Rv

H37Ra

HEK

HIP

HTS

HOP
Acetone.

Absorbance units.

Broad.

1,1' Carbonyldiimidazole.

Colony forming units.

Doublet.

Diode array detector.

Dichloromethane.

4-(Dimethylamino) pyridine.

Doublet of doublets.

Doublet of doublet of doublets.

2,3-Dihydroxy-propoxypropyl-derivatised silica gel.

Dimethylformamide.

Dimethylsulfoxide.

Directly observed therapy short course.

$N$-(3-Dimethylaminopropyl)- $N$ '-ethylcarbodiimide

hydrochloride.

Epidermal growth factor receptor.

Extended-spectrum beta-lactamase producing Enterobacteriaceae.

Ethyl acetate.

Fluorescence activated cell sorting.

Fatty acid methyl esters.

Free fatty acid.

Gas chromatography with flame ionisation detection.

Green fluorescent protein.

Hexanes.

virulent disease causing strain of $M$. tuberculosis.

avirulent non-pathogenic strain of $M$. tuberculosis.

Human embryonic kidney.

Haploinsufficiency profiling.

High-throughput screen.

Homozygous deletion profiling. 
HP20/HP20ss

HRESIMS

HSQC

IA

IHD

IPTG

LB

LOPAC

MDR

$\mathrm{MeCN}$

$\mathrm{MeOH}, \mathrm{M}$

MIC

$\mathrm{MIC}_{90}$

MNP

MOA

MQ

MRSA

MS

MTPA

MTT

NA

OADC

PBS

PC-2

PKC

PKS

PLA2

PSDVB

RFU

SAR

SC

SDS

SGA
Poly(styrene-divinylbenzene) stationary support.

High-resolution electrospray ionisation mass spectrometry.

Heteronuclear single-quantum coherence $\left({ }^{1} \mathrm{H}\right.$ to $\left.{ }^{13} \mathrm{C}\right)$.

Inactive.

Index of hydrogen deficiency.

Isopropyl $\beta$-D-1-thiogalactopyranoside.

Luria-Bertani broth.

Library of Pharmacologically Active Compounds.

Multi-drug resistant.

Acetonitrile.

Methanol.

Minimum inhibitory concentration.

Minimum inhibitory concentration at which $90 \%$ inhibition is observed.

Marine natural product.

Mechanism of action.

Milliq purified distilled water.

Methicillin resistant Staphylococcus aureus.

Mass spectrometry.

$\alpha$-Methoxy- $\alpha$-trifluoromethylphenylacetic acid.

3-(4,5-Dimethylthiazol-2-yl)-2,5(diphenyltetrazolium bromide).

Not achievable.

$(0.06 \%$ oleic acid, $5 \%$ BSA, $2 \%$ Dextrose, $0.85 \% \mathrm{NaCl})$.

Phosphate-buffered saline.

Physical containment level 2.

Protein kinase $\mathrm{C}$.

Polyketide synthase.

Phospholipase A2.

Poly(styrene-divinylbenzene).

Relative fluorescence units.

Structure-activity relationship.

Synthetic complete broth.

Sodium dodecyl sulphate.

Synthetic genetic array. 
SLAM

TB

TDR

TI

TOCSY

VUW

XDR
Synthetic lethality analysed by microarray.

Tuberculosis.

Totally drug resistant.

Therapeutic index.

Total correlation spectroscopy $\left({ }^{1} \mathrm{H}\right.$ to $\left.{ }^{1} \mathrm{H}\right)$.

Victoria University of Wellington.

Extensively drug resistant. 


\section{List of publications}

Nathaniel Dasyam, Andrew B. Munkacsi, Nazmi H. Fadzilah, Dinindu S. Senanayake, Ronan F. O'Toole, Robert A. Keyzers. Identification and bioactivity of 3-epixestoaminol C isolated from the New Zealand brown alga Xiphophora chondrophylla. Journal of Natural Products, 2014, ACCEPTED, DOI: 10.1021/np500171z.

\section{Expected}

Nathaniel Dasyam, Ronan F. O’Toole, Robert A. Keyzers. Identification, synthesis and bioactivity of rimarikiamide A isolated from a Latrunculia sp. New Zealand sponge. Journal of Natural Products, in preparation.

Nathaniel Dasyam, Ronan F. O'Toole, Robert A. Keyzers. Anti-mycobacterial fatty acids from the liver extract of Psychrolutes marcidus, Marine Drugs, in preparation. 
Chapter 1 Introduction 


\subsection{Tuberculosis}

\subsubsection{Disease}

Mycobacterium tuberculosis was first identified as the cause of tuberculosis (TB) by Robert Koch in 1882 [1]. TB is an ancient disease, with evidence of its persistence through time recorded in the remains of 3000 year old ancient Egyptian populations [2]. The earliest evidence of humans infected with TB can be dated back to a 9,000 year old Neolithic settlement in the Eastern Mediterranean [3].

TB primarily affects the lungs, with common symptoms such as fever, night sweats, fatigue, coughing, in severe cases bloody and weight loss [4]. Without appropriate treatment $70 \%$ of pulmonary TB infections are terminal [5]. Extra-pulmonary TB is a form that infects any part of the body other than the lungs including the skin, the central nervous system and the reproductive organs [6]. The most severe form of extrapulmonary $\mathrm{TB}$ is miliary $\mathrm{TB}$, which occurs when bacteria infiltrate blood circulation and can affect multiple organs [7]. This form of the disease has a high mortality rate, $15-20 \%$ in children and $25 \%-50 \%$ in adults [7].

\subsubsection{Disease statistics}

While the overall mortality rates associated with TB is decreasing, in 20128.6 million cases of TB and 1.3 million deaths due to TB were reported by the World Health Organisation (WHO) [8]. Controlling this disease is hindered by the latent form of TB, which is currently believed to affect a third of the world population [9]. In latent TB infection bacilli stay dormant and do not cause active disease, individuals with this form of TB have a $10 \%$ risk of developing active TB during their lifetime, each of whom will go on to infect 10-15 people [9]. In New Zealand (NZ) a total of 661 cases of TB were identified in 2010 [10]. NZ has a much higher incidence of TB (6.6 per 100, 000) compared to Australia and the United States of America [10]. Among cases of NZ born patients over $50 \%$ were of Maori ethnicity compared to $19 \%$ for patients of European ethnicity [10]. 


\subsubsection{Mycobacteria}

Mycobacteria are rod shaped non-motile bacteria that can be differentiated using acid fast staining. The acid fastness is unique, due to the high mycolic acid content in the cell walls of mycobacteria, which retain the carbol-fuchsin dye after decolourising with acidic alcohol $[11,12]$. These bacteria are widespread in diverse environments ranging from soil to water [13], and are generally categorised into two groups, fast-growing and slow-growing. Fast growing mycobacteria show defined colonies on agar within seven days of inoculation [14]. These include M. smegmatis, M. fortuitum and M. abscessus, which are responsible for post-surgical as well as skin and soft tissue infections [15]. This group also includes $M$. phlei and $M$. aurum, which are generally considered as non-infectious mycobacteria, although there have been reported cases of these strains causing disease in immunocompromised patients $[16,17]$.

Slow-growing mycobacteria take longer than seven days to show visible growth [14], this group includes $M$. tuberculosis, M. bovis, M. africanum and M. leprae, which belong to the Mycobacterium tuberculosis complex (MTB complex), referring to a group of mycobacteria that can cause tuberculosis in either human or animal hosts. Other slow growers include $M$. avium, M. kansasii and M. marinum all of which cause varying types of skin lesions [15].

Details of two species of mycobacteria that were utilised in this research are described below.

\subsubsection{Mycobacterium smegmatis}

This species of mycobacteria is widespread in soil [18], although generally considered to be non-pathogenic it can be an opportunistic pathogen resulting in post-surgical infections [19]. Due to its low pathogenicity as well fast replication time, this species is the work horse for mycobacterial research [20]. The ease of genetic manipulation as well as similarities in drug susceptibility to pathogenic mycobacteria make M. smegmatis an appropriate model organism for studying clinically relevant mycobacteria $[21,22]$. 


\subsubsection{Mycobacterium tuberculosis}

Mycobacterium tuberculosis H37Rv and H37Ra were both derived from their virulent parent strain H37 [23]. As such, H37Ra shares a high similarity to the virulent strain $\mathrm{H} 37 \mathrm{Rv}$, although as a result of 53 insertion and 21 deletion events, H37Ra has a genome 8,445 bp larger than H37Rv [23]. In addition, the genome of H37Ra differs even further due to 76 single nucleotide variance in 32 genes. These genetic variances are believed to account for the avirulence of H37Ra, the difference in colony morphology and decreased survival inside macrophages as well as under anaerobic conditions [23]. Although H37Rv is considered the standard laboratory strain, the lack of pathogenicity of H37Ra makes it an ideal model of TB that can be used in PC2 facilities.

\subsubsection{Disease and pathogenesis}

TB spreads through aerosols when an infected individual coughs, spits or sneezes [9]. Upon entry into the lung, bacilli are engulfed by resident alveolar macrophages, although research has also identified that other phagocytic cells such as dendritic cells and neutrophils are also responsible for the uptake of bacteria [24]. This process is mediated through several different receptors that recognise and bind to mycobacterial cell wall components [24]. M. tuberculosis can modulate the host immune response in multiple ways, one of which is preventing the maturation of the phagosome [24]. Further modulation by bacilli ensure that necrosis rather than apoptosis is induced in infected host cells, which recruits more non-infected macrophages and facilitates further replication of bacilli [24].

The uptake of bacilli and migration of infected macrophages to a different site is followed by the concomitant formation of granulomas, which is a clear sign of tuberculosis pathology in patients [24]. Within a granuloma, activated macrophages and other inflammatory cells destroy everything including host tissue in order to control intracellular growth of bacilli, this is termed caseous necrosis [25]. It should be noted that not all mycobacteria are destroyed within granulomas, instead a state of balance between the host defence and pathogen's progress is met [24]. By recruiting immune cells the host is able to control the spread of bacteria, while the bacilli are able to use the newly recruited immune cells to replicate [24]. 
The connection of caseous necrotic tissue to the airway within a patient is what leads to active TB. The change from anoxic conditions to an oxygen rich environment leads to an increase in bacterial growth and eventually patient mortality if left untreated [25]. When caseous necrotic tissue that contains dormant bacilli are released into the airway, bacilli are able to spread to other areas of the lung and start replicating extracellularly [25]. It is at this point where the disease becomes contagious, as bacteria are now able to be spread when infected individuals cough or sneeze [26]. Newly exposed bacteria are taken up by macrophages, through necrosis these infected macrophages eventually destroy neighbouring tissue and create a new area of caseous tissue. Cycles of increased infection followed by resolution forms scar tissue, causing permanent damage to the lung leading to mortality [25]. The ability of M. tuberculosis to modulate the immune system and proliferate within immune cells highlights the reason behind the resilience of the bacteria through time. Fortunately, effective treatments exist to manage a TB infection.

\subsection{Current treatment regimen}

Direct observed therapy short course (DOTS) is the current standard treatment for TB. DOTS lasts for six months to treat drug susceptible TB and can take up to 20 months to treat multi-drug resistant TB (MDR-TB) [27]. DOTS can be either universal or selective; in selective DOTS the treatment can be self-administered, whereas in universal DOTS the entire course of treatment is monitored by health professionals [28]. A recent study comparing the two methods has indicated that universal DOTS results in far less occurrence of drug resistant TB arising within patients, with isolates from selective DOTS being five times more likely to be resistant [28].

DOTS consists of an intensive and a continuation phase. The intensive phase lasts for two months, using a combination of pyrazinamide, rifampicin, ethambutol and isoniazid. This is followed by four months of the continuation phase using a combination of isoniazid and rifampicin [27].

Rifampicin (1) is a bactericidal drug that inhibits transcription through its interaction with the DNA dependent RNA-polymerase $\beta$ subunit [29]. Pyrazinamide (2) is believed to disrupt the membrane potential and decrease intracellular $\mathrm{pH}$, leading to inactivation of many important cellular pathways [30]. Ethambutol (3) works to inhibit arabinosyl 
transferases that are important for cell wall synthesis [31], while isoniazid (4) inhibits cell wall synthesis as well, but does so by inhibiting fatty acid synthesis [32]. The use of a combination of drugs with different modes of action reduces the risk of drug resistance arising as well as limits the chance of relapse among patients [33, 34].

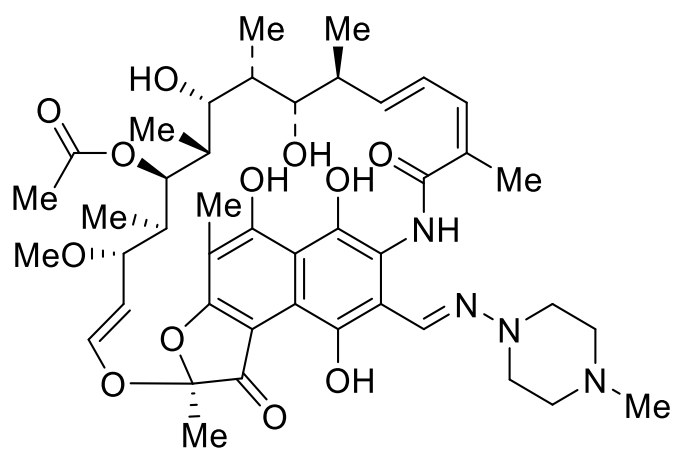

1<smiles>NC(=O)c1cnccn1</smiles>

2<smiles>CCC(CO)NCCNC(CC)CO</smiles>

3<smiles>NNC(=O)c1ccncc1</smiles>

4

Patient non-adherence, poor treatment management due to lack of funding, overburdened health systems as well as socioeconomic factors all contribute to ineffective application of DOTS and consequently the rise of drug resistance [35, 36]. The treatment of tuberculosis is compounded by the effects of Human Immunodeficiency Virus (HIV) [35]. HIV positive patients are more likely to develop TB and have a higher rate of mortality [37]. Drug-drug interactions are the main issue when treating co-infected individuals. The use of rifampicin becomes problematic in situations where patients are taking either protease inhibitors or non-nucleoside reverse transcriptase inhibitors for HIV, as well as receiving DOTS [38]. Rifampicin causes an increase in CYP3A4, a cytochrome P450 enzyme, which decreases the plasma levels of the antiretroviral drugs, thus limiting their activity [39]. These types of complex drug-drug interaction must be addressed and stress the need for more effective treatments. 
Table 1.1 Current first and second line anti-tuberculosis drugs

\section{Mechanism of action}

\section{First-line drugs}

Isoniazid (4) Inhibits mycolic acid synthesis by targeting Enoyl-[acyl-carrierprotein] reductase

Rifampicin (1) Inhibits transcription by targeting RNA polymerase beta subunit

Pyrazinamide (2) Inhibits translation and trans-translation, acidifies cytoplasm targets S1 component of 30S ribosomal subunit.

Ethambutol (3) Inhibits arabinogalactan biosynthesis by targeting arabinosyl transferases

\begin{tabular}{|c|c|}
\hline $\begin{array}{l}\text { Second-line } \\
\text { drugs }\end{array}$ & \\
\hline $\begin{array}{l}\text { Para-amino } \\
\text { salicylic acid (5) }\end{array}$ & $\begin{array}{l}\text { Inhibits folate biosynthesis by inhibiting dihydropteroate } \\
\text { synthase }\end{array}$ \\
\hline Streptomycin (6) & $\begin{array}{l}\text { Inhibits protein synthesis by inhibiting } \mathrm{S} 12 \text { and } 16 \mathrm{~S} \text { rRNA } \\
\text { components of the } 30 \mathrm{~S} \text { ribosomal subunit }\end{array}$ \\
\hline Ethionamide (7) & $\begin{array}{l}\text { Inhibits mycolic acid biosynthesis by targeting Enoyl-[acyl- } \\
\text { carrier-protein] reductase }\end{array}$ \\
\hline Ofloxacin (8) & $\begin{array}{l}\text { Inhibits DNA supercoiling by targeting DNA gyrase and } \\
\text { topoisomerase }\end{array}$ \\
\hline Capreomycin (9) & $\begin{array}{l}\text { Inhibits protein synthesis forming an interbridge } \mathrm{B} 2 \mathrm{a} \text { between } \\
\text { the } 30 \mathrm{~S} \text { and } 50 \mathrm{~S} \text { ribosomal subuints. }\end{array}$ \\
\hline Kanamycin (10) & $\begin{array}{l}\text { Inhibits protein synthesis by targeting the } 30 \mathrm{~S} \text { ribosomal } \\
\text { subunit }\end{array}$ \\
\hline Amikacin (11) & $\begin{array}{l}\text { Inhibits protein synthesis by targeting the } 30 \mathrm{~S} \text { ribosomal } \\
\text { subunit }\end{array}$ \\
\hline Cycloserine (12) & $\begin{array}{l}\text { Inhibits peptidoglycan synthesis by targeting D-alanine } \\
\text { racemase and ligase }\end{array}$ \\
\hline
\end{tabular}

Tuberculosis treatment drugs and their primary mechanism of action. Table adapted with permission from Cole et al. [40]. 
<smiles>Nc1ccc(C(=O)O)c(O)c1</smiles>

5<smiles>CCc1cc(C(N)=S)ccn1</smiles>

7

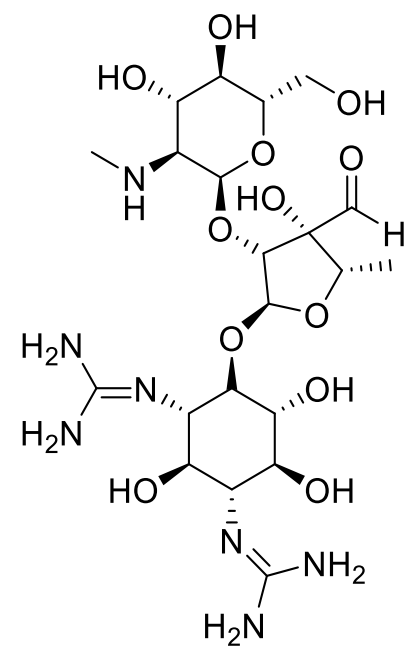

6

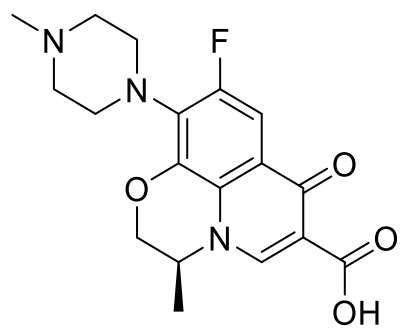

8

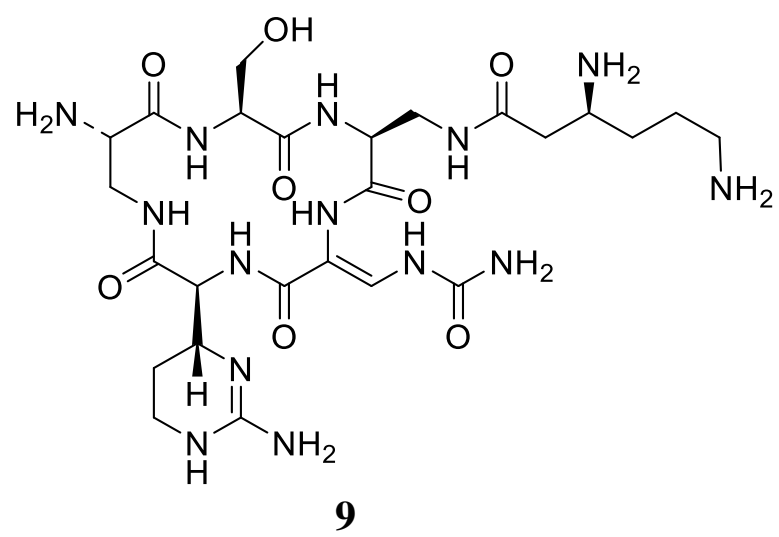

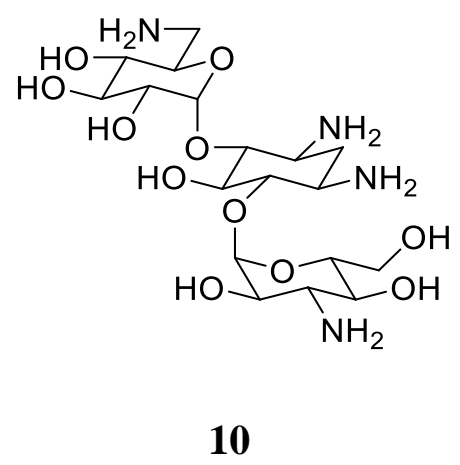




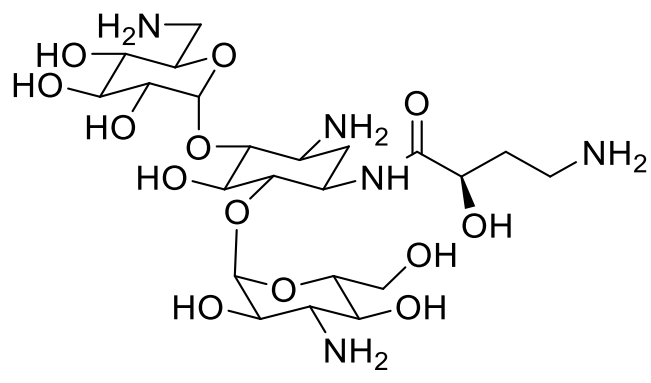

11<smiles>N[C@@H]1CONC1=O</smiles>

12

\subsubsection{Vaccination}

At present, the only way to prevent a TB infection is the use of the live attenuated strain M. bovis BCG (Bacillus Calmette-Guerin) as a vaccine [41]. It is derived from virulent M. bovis, which primarily infects cattle [42]. Thirteen years of passaging cultures of M. bovis led to developing BCG and the vaccine has been shown to reduce the risk of TB by $50 \%[42,43]$. Furthermore, BCG also decreases the risks of developing more harmful forms of TB such as miliary TB and TB meningitis [7]. Until a more effective vaccine can be developed chemotherapeutics continue to be the most effective control for TB.

\subsection{Drug resistance}

The discovery of 6 in 1943 was believed to herald the end of this persistent disease [44], however this was not to be, with resistance evident as early as the first clinical trial [44]. TB is now established all around the world and drug resistant forms of the disease are widespread [9]. Drug resistance can occur through many pathways including drug inactivation, modification of the drug target, drug efflux pumps or by using alternative pathways to overcome drug mediated effects [45](Table 1.2). In addition, the high mycolic acid content of mycobacterial cell walls protect the bacilli by decreasing the permeability to chemotherapeutic agents [46]. 
Table 1.2 Common mechanisms of drug resistance to first line anti-TB drugs

\begin{tabular}{|c|c|}
\hline Drug & Mechanism of resistance \\
\hline Isoniazid & $\begin{array}{l}\text { Mutation within the gene katG [47], through its } \\
\text { peroxidase and catylase activity KatG is normally } \\
\text { responsible for the activatation of the pro-drug [48]. } \\
\text { Other mutations such as ahpC and inhA have also been } \\
\text { linked to isoniazid resistance [47]. }\end{array}$ \\
\hline Rifampicin & $\begin{array}{l}\text { Resistance is linked to mutations in the } r p o B \text { gene at } \\
\text { codon positions } 531,526 \text { and } 516 \text {, which affects the } \\
\text { binding of rifampicin to its target binding site in the } \beta \\
\text { subunit of RNA polymerase [49]. }\end{array}$ \\
\hline Pyrazinamide & $\begin{array}{l}\text { As the exact target has not been identified, the } \\
\text { consequent mechanism of pyrazinamide resistance has } \\
\text { not been fully elucidated, although mutations within the } \\
\text { pncA gene, which is believed to activate the pro-drug, } \\
\text { have been implicated in resistance [50]. }\end{array}$ \\
\hline Ethambutol & $\begin{array}{l}\text { Mutation within the embCAB operon, with mutations in } \\
e m b \mathrm{~B} \text {, which encodes for an arabinosyl transferase, } \\
\text { accounting for most cases of resistance [51]. }\end{array}$ \\
\hline
\end{tabular}

The current definitions of drug resistance in TB include: mono-resistance, polydrug resistance, rifampicin resistance, multi drug resistance and extensive drug resistance [52]. Mono-resistance refers to resistance against only one first-line anti-TB drug, while polydrug resistance is resistance to more than one first-line drug that does not include rifampicin or isoniazid [52].

Multi-drug resistant TB (MDR-TB) refers to strains resistant against multiple drugs but has to include resistance to rifampicin and isoniazid [52]. For extensive drug resistant TB (XDR-TB), in addition to the resistance profile of MDR-TB, the strain should also 
display resistance to any fluoroquinolone and at least one of the second-line injectable drugs [52]. Rifampicin resistance includes any strain that displays resistance to rifampicin and may include mono, poly, multi or extensive drug resistant strains [52].

Compared to the cure rate for drug susceptible TB $(85-87 \%)$ the cure rate for MDR-TB is much lower at 48\% [8]. Reports of MDR-TB cases started to rise in the 1990s [53, 54]. Approximately 450,000 cases of MDR-TB were reported in 2012, 9.6\% of which were XDR-TB [8]. Recently, the emergence of a new strain unofficially termed totally drug resistant TB (TDR-TB) has been reported from Iran, India and Italy, this further highlights the threat that TB still imposes upon society [55-57]. TDR-TB has displayed resistance to all first and second line drugs, making a TDR-TB infection nearly impossible to cure with current drugs. The need for a new classification for this emerging strain has not yet been deemed necessary by the WHO [58].

MDR-TB strains were first reported in the 1990s [59], their required treatment uses drugs that are more expensive and have more adverse side effects [60]. XDR-TB was first detected in strains of TB collected between 2000-2004 [59]. This form of the disease is even more difficult to cure and if patients are immunocompromised they have much higher chance of mortality, which can be as high as 98\% [61]. With a treatment efficacy as low as $20 \%$ for XDR-TB, patients have a much lower chance for survival [8]. In New Zealand, there have been a total of 28 MDR- TB cases in the past 13 years, as well as one reported case of XDR-TB in 2010 [62].

MDR and XDR-TB as well as the emergence of TDR-TB stress the need for better treatments for TB now more than ever before. The promising drug candidates listed below highlight the response to address the need for new and more effective drugs. These include the new class of anti-mycobacterial compounds, PA-824 (13) and OPC-67683 (14), nitroimidiazole drugs, which are in Phase II and III clinical trials, respectively [40]. These drugs are active against both replicating and non-replicating bacteria. When these drugs undergo reduction, damaging reactive nitrogen species are produced, resulting in the observed inhibitory activity [63]. 
<smiles>O=[N+]([O-])c1cn2c(n1)OCC(OCc1ccc(OC(F)(F)F)cc1)C2</smiles>

13<smiles>C[C@]1(COc2ccc(N3CCC(Oc4ccc(OC(F)(F)F)cc4)CC3)cc2)Cn2cc([N+](=O)[O-])nc2O1</smiles>

14

SQ-109 (15) is an ethylenediamine analogue of ethambutol (3), with a differing mode of activity. The current proposed target for this drug is the trehalose monomycalate transporter, which supplies mycolic acid during cell wall synthesis [64].<smiles>CC(C)=CCC/C(C)=C/CNCCNC1C2CC3CC(C2)CC1C3</smiles>

15

PNU-100480 (16), an oxazolidinone, is a derivative of linezolid that inhibits protein synthesis by binding to the $23 \mathrm{~S}$ rRNA within the $50 \mathrm{~S}$ ribosomal subunit [65]. This drug is currently under Phase II clinical trials [40].<smiles>CC(=O)NC[C@H]1CN(c2ccc(N3CCSCC3)c(F)c2)C(=O)O1</smiles>

16

The final example highlights a drug that has passed through the drug development process and has gained orphan drug status to treat MDR and XDR-TB. The diarylquinoline TMC-207 (17, bedaquiline) inhibits the ATP-synthase subunit $\mathrm{c}$ and was approved by the FDA in December $2012[66,67]$. 


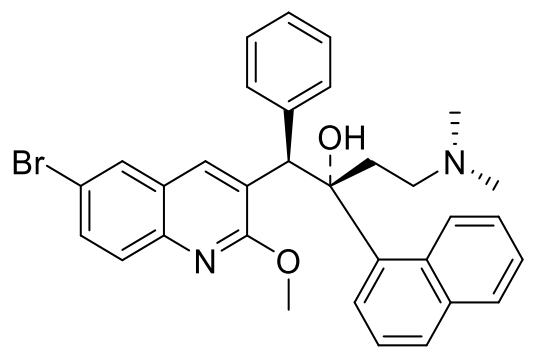

17

Even with the wealth of novelty in the current pipeline [40], the high attrition rate of new candidate drugs undergoing the drug approval process, requires a constant supplement to the pipeline with new potent compounds [68]. Natural products can provide a source of such compounds.

\subsection{Natural product chemistry}

Natural product chemistry tends to focus on the identification of novel secondary metabolites $[69,70]$. By definition, these metabolites are not essential for the viability of the organism, rather they may aid the organism by providing a competitive advantage [71]. It has been postulated that some of these compounds may be produced by symbiotic bacteria, as is the case for bryostatin-1, which is currently under clinical trials for its role as an anti-cancer agent and also as a treatment for Alzheimer's disease [69]. The bryostatins were first isolated from the bryozoan Bugula neritina, however the genes for the biosynthesis of this compound can be identified in the symbiotic bacterium Candidatus Endobugula sertula [72]. In plants, some compounds isolated are produced by endophytic fungi, as in the case of taxol [73]. The biogenesis of these metabolites usually employ alternative pathways to primary metabolism such as the non-ribosomal peptide synthetase or polyketide synthetase pathways [74]. These pathways are open to modifications by molecular biology tools and can also be used as genetic markers to identify microbes that may be potential sources of unique compounds $[69,75]$.

\subsubsection{Terrestrial sources}

Throughout history natural products have provided treatments for ailments with the earliest record for the use of natural remedies being documented on clay tablets from Mesopotamia dating back to 2600 BC [76]. Morphine represents the first commercial 
pure drug and penicillin the first antibiotic [76]. Approximately 74\% of all approved antibacterial drugs identified in the 1981-2010 time frame originated from natural products [77]. This highlights the wealth of natural products and reveals their potential for the future.

\subsubsection{Natural products as anti-TB drugs}

The potential of natural products for the treatment of TB can be highlighted by the fact that many of the current TB drugs that have been used for decades originated as a result of natural product research. Streptomycin, the first drug that was used to fight TB, was isolated from Streptomyces griseus [78]. Isoniazid and pyrazinamide are examples of two very effective first line drugs, which were identified due to their structural similarities to the natural product nicotinamide that demonstrated anti-tubercular effects [79]. Rifamycin, a polyketide isolated from the bacterium Amycolatopsis rifamycinica, provided a lead to rifampicin, a gold standard anti-tubercular compound that is currently used for its bactericidal activity [80, 81].

p-Aminosalicylic acid, a second line drug, was a product of research that observed the natural product salicylic acid increased oxygen uptake in M. tuberculosis; this led to screening of analogues of salicylic acid to identify competitive inhibitors in the pathway that salicylic acid was interacting with [82]. Cycloserine, another second line drug, is a cell wall synthesis inhibitor and was first isolated from Streptomyces orchidaceus [83, 84]. The second line aminoglycoside drug kanamycin, a protein synthesis inhibitor, which later provided the lead for the protein synthesis inhibitor amikacin, was isolated from Streptomyces kanamyceticus [85]. Capreomycin, a cyclic peptide often grouped with the aminoglycosides, is another protein synthesis inhibitor that was isolated from Streptomyces capreolus [86].

Given the fact that more than nine of the current anti-tubercular drugs have originated from natural products, it is appreciable that they provide a beneficial source for future anti-tubercular drugs. However considering rifampicin, the most recent first line anti-TB drug, was discovered nearly 55 years ago [87], there is a significant demand to find new and more effective drugs. 


\subsubsection{Marine environment}

Scientists have searched the terrestrial environment for antibiotics since the serendipitous discovery of penicillin in 1928 [88]. Researching un-investigated environments may prove useful in identifying novel chemical scaffolds to provide leads for efficacious drugs. In 2011, 1152 new compounds were reported from marine organisms [89]. The marine environment contains organisms producing an array of distinctive molecules, which can be illustrated by the abundance of such structures reported by researchers [89].

From the coasts of New Zealand, specific lipids isolated from the green lipped mussel may provide an effective treatment for asthma and other inflammatory diseases [90, 91]. Peloruside A (18) was isolated from the sea sponge Mycale hentscheli collected in the Marlborogh Sounds of New Zealand. This metabolite shows anti-mitotic activity, making it of interest in the study for anti-cancer therapies [92].

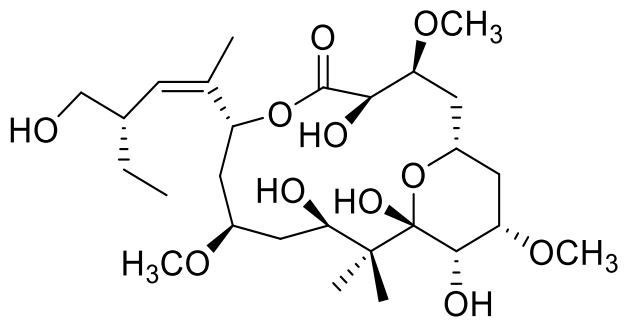

18

Another interesting discovery was from Conus magus, a cone snail that paralyses its prey using a poison tipped barb. From this poison, a painkiller called ziconotide (19), was developed and is currently marketed as Prialt for the treatment of chronic pain. This was the first marine drug to be approved by the FDA [93]. From Ecteinascidia turbinata, a sea squirt, a compound ecteinascidin 743 (20) that PharmaMar has brand-named Yondelis ${ }^{\circledR}$ has gained approval from the European Medicines Agency for its anti-tumor activity against soft-tissue sarcomas and ovarian cancer [69].

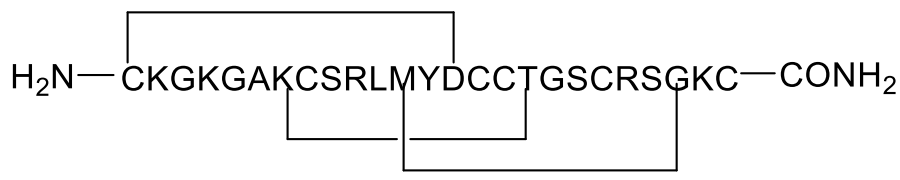

19 


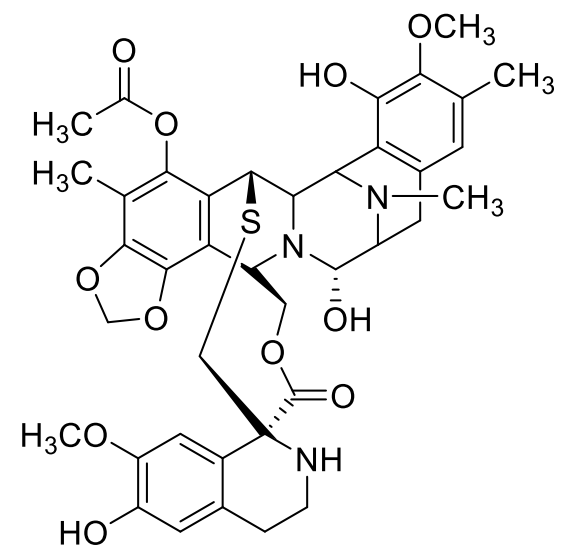

20

A more recent addition to the list of approved drugs that have been derived from the marine environment is Eribulin (halaven, 21 ), which gained approval for the treatment of metastatic breast cancer [94]. This compound is a synthetic analogue of halichondrin B originally isolated from the Japanese sponge Halichondria okadai [95].

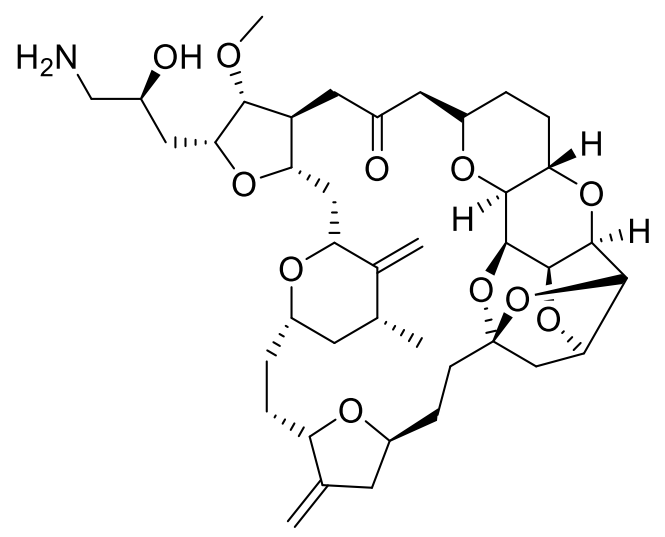

21

More recently, Jensen and Fenical have worked on a marine microbiology discovery program and have discovered a group of obligate marine actinomycetes belonging to the genus Salinospora [96]. There are now well above 2000 strains of this genus identified [97]. With this wealth of biodiversity one can imagine the potential to identify unique new compounds. This potential is reinforced by the fact that there are currently 13 marine chemical entities at different stages of clinical trials, with the majority focusing towards treatments for cancer [98]. 


\subsubsection{Marine natural products as anti-tubercular agents}

In literature pertaining to the TB field, it seems that most active marine extracts tend to come from sea sponges, most probably due to the fact these organisms contain vast numbers of symbionts [99]. Some novel structures that have been identified to inhibit mycobacteria include homopseudopteroxazole (22), a diterpene alkaloid isolated from the Caribbean gorgonian octocoral Pseudopterogorgia elisabethae [100]. Halicyclamine A (23), obtained from an Indonesian sea sponge Haliclona sp. has shown bactericidal activity against $M$. bovis, M. smegmatis and M. tuberculosis [101]. From the Caribbean sponge Svenzea zeai, two abeo-sterols, parguesterol A (24) and B (25) were isolated with reported MIC values of 7.8 and $11.2 \mu \mathrm{g} / \mathrm{mL}$ against $M$. tuberculosis $\mathrm{H} 37 \mathrm{Rv}$ as well as reported $\mathrm{IC}_{50}$ values of $52 \mu \mathrm{g} / \mathrm{mL}$ in Vero cells for both compounds [102].<smiles>CCCCCc1nc2c(C)c3c4c(c2o1)[C@@H](C)CC[C@H]4[C@H](C)C[C@H]3C=C(C)C</smiles>

22

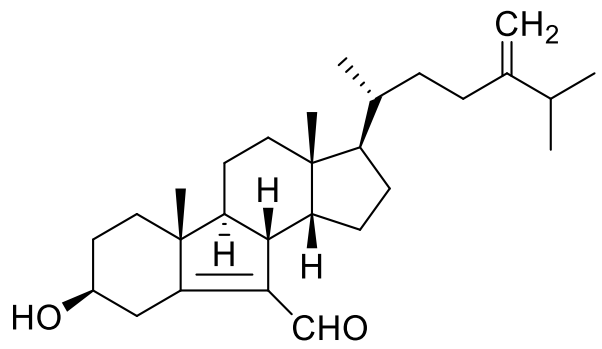

24

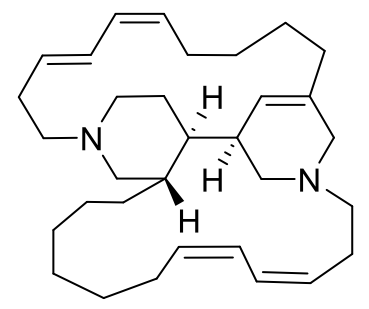

23

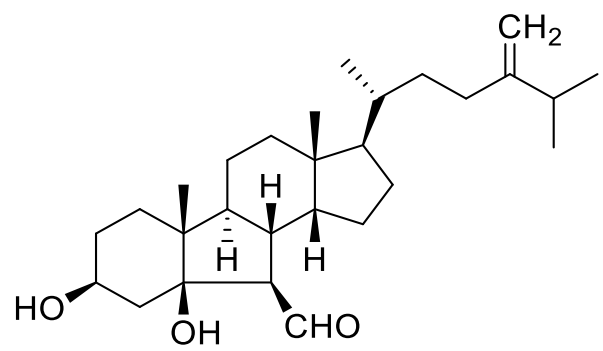

25

Pseudopyronines A (26) and B (27), isolated from a marine Pseudomonas. sp F92S91 [103], displayed inhibitory activity against M. tuberculosis, with pseudopyronine B a greater inhibitior of InhA, a fatty acid biosynthesis enzyme [104]. Manzamine A (28) isolated form a Haliclona sp. sponge [105], and manzamines E (29) and F (30) isolated 
from an Okinawan Xestospongia sp sponge [106], displayed respective MIC values of 1.53, 3.76 and $2.56 \mu \mathrm{g} / \mathrm{mL}$ against $M$. tuberculosis H37Ra [107].

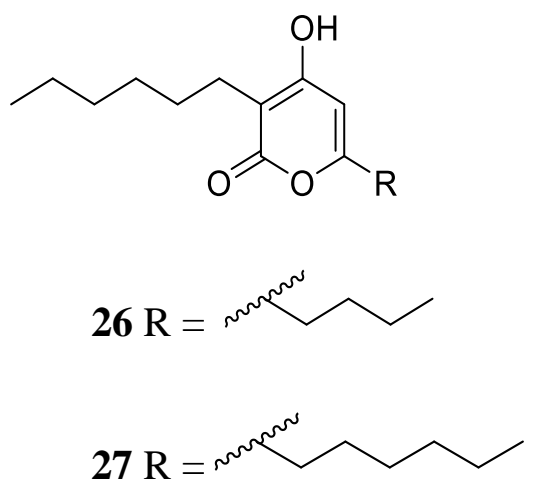

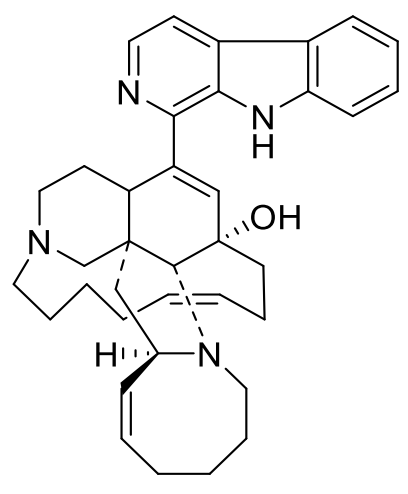

28

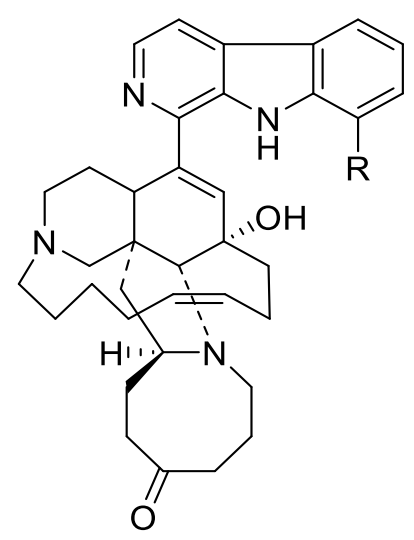

$29 \mathrm{R}=\mathrm{H}$

$30 \mathrm{R}=\mathrm{OH}$

The marine fungus Aspergillus versicolor was isolated from a marine sediment from the Bohai Sea, China, which led to the identification of brevianamide S (31), this compound displayed an MIC of $6.25 \mu \mathrm{g} / \mathrm{mL}$ against BCG [108]. Trichoderins A (32), A1 (33) and B (34) were isolated from a Trichoderma sp., a marine sponge-derived fungus with MIC values of $0.12,2.0$ and $0.13 \mu \mathrm{g} / \mathrm{mL}$ respectively against $M$. tuberculosis $\mathrm{H} 37 \mathrm{Rv}$ [109]. Abyssomicin C (35) and atrop-abyssomicin C (36), isolated from a marine Verrucosispora strain AB-18-032 [110], displayed MIC values of 3.6 and $7.2 \mu \mathrm{M}$ against M. tuberculosis H37Rv [111]. 


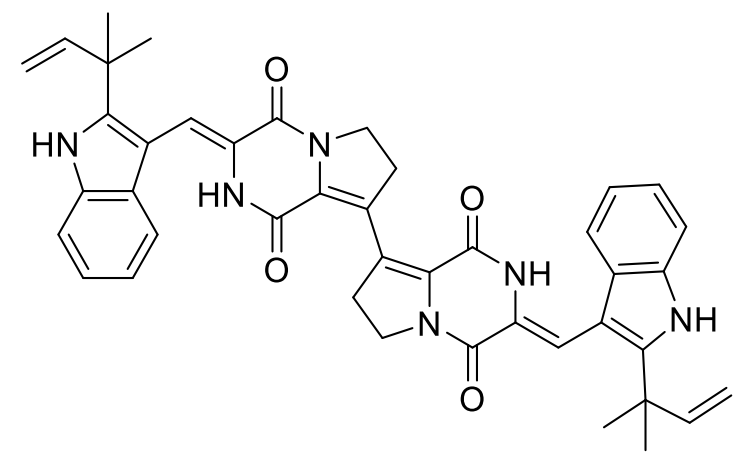

$C_{O}^{C}$<smiles>[R2]=C[14CH2][C@H](O)[C@H](O)CC</smiles>

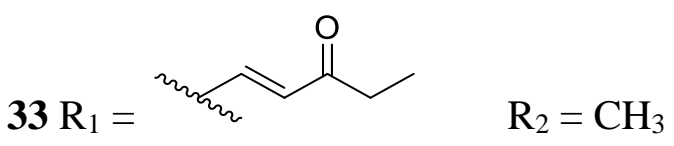<smiles>[R][R4]#CC[C@@H](O)CC(=O)CC</smiles>

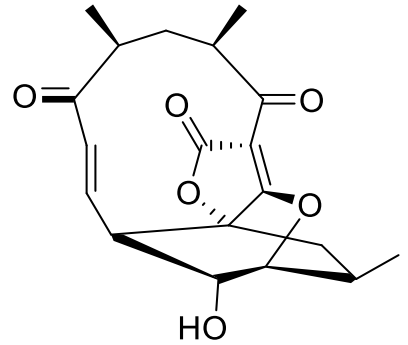

35

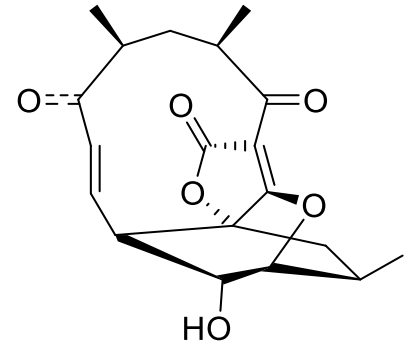

36 
Considering the growth of marine microbial research, it is believed that scientists have only studied a small fraction of this valuable environment for structurally novel compounds [69]. The function of these compounds could be vital by providing cures or bio-probes to ascertain important information on biological processes [93].

\subsubsection{Screening for natural products}

\subsubsection{Bioassay-guided fractionation}

The classical approach to natural product isolation has been bioassay-guided fractionation [112]. The serendipitous isolation of penicillin heralded the era of bioassay-guided fractionation, an approach that has never ceased to be a valuable resource for finding bioactive compounds. This approach involves screening crude extracts for activity, following which fractionation is performed on an active sample. The subsequent fractions are tested for bioactivity, the most active fractions are further purified and tested again, and the process is repeated in iterative fashion until the bioactive compounds have been isolated. The purities of the fractions are monitored using spectroscopic methods. This approach requires a set drug target or desired biological activity or a pathway to investigate, with a well-designed and optimised assay. These assays may be simple whole cell assays looking for growth inhibition of clinically relevant pathogens [69], or more complex biochemical assays that may require extensive optimizations prior to screening [113]. This technique is considered destructive as each bioassay requires the expenditure of compound. Another common issue with this classical approach is that it often leads to the isolation of known compounds, especially if the source of the extract has been explored in earlier research [114]. Furthermore this approach can lead to novel compounds being completely ignored as they maybe inactive in the assay system being employed. In some cases, compounds with detergent-like or promiscuous activity are identified as hits and are undesired as they are often cytotoxic and do not make ideal drug leads [115]. In rare cases, activity may be due to two or more compound working in synergy or with additive effects, further fractionation in such situations can lead to a loss of activity. The strength in this approach lies in the fact that a bioactivity of interest is able to be pursued throughout all stages of isolation. To avoid the issue of re-isolation, this approach can be complimented with spectroscopic analysis to prevent further fractionation of extracts with chemical profiles of known compounds [114]. 


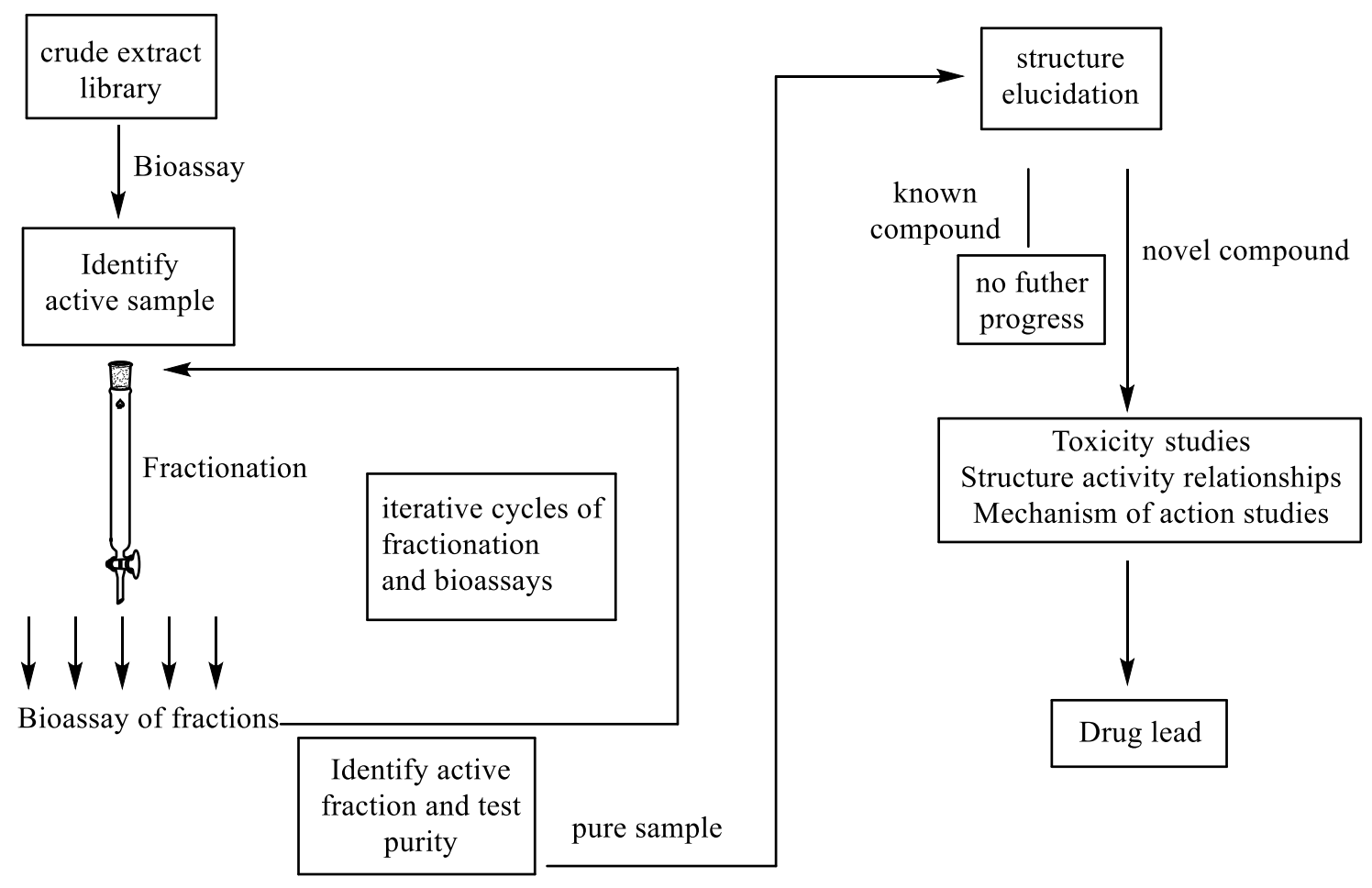

Figure 1.1 Bioassay-guided fractionation

The basic approach to bioassay-guided fractionation for novel compounds.

\subsubsection{Structure-guided isolation}

An alternative approach to bioassay-guided fractionation is structure-guided isolation. This approach is often employed in groups where applying a bioassay after each point of fractionation is often not feasible either due to lack of expertise or lack of appropriate facilities. The robustness of this approach can be observed by the fact that throughout the fractionation process, the compound of interest can able to be tracked using either nuclear magnetic resonance (NMR) spectroscopy, liquid chromatography coupled to mass spectroscopy (LC-MS) or high performance liquid chromatography linked to a diode array detector (DAD-HPLC). In essence, the bioassay is replaced by an analytical instrument such as a NMR spectrometer and rather than bioactivity, the chemical signatures of unusual secondary metabolites are tracked. When dealing with small levels of compound, highly sensitive spectrometers are required. The downfall of this approach is that since activity was never the primary goal, large masses of compound must be sacrificed to different assays in order to identify the compound's bioactivity. If the compound of interest has been isolated in low quantities this may not allow for it to 
be studied in enough suitable assays. This expenditure of compound in multiple assays can be circumvented if the compound is used in an assay that can monitor its effects on a genome wide scale, such as the Saccharomyces cerevisiae deletion mutant library [116].
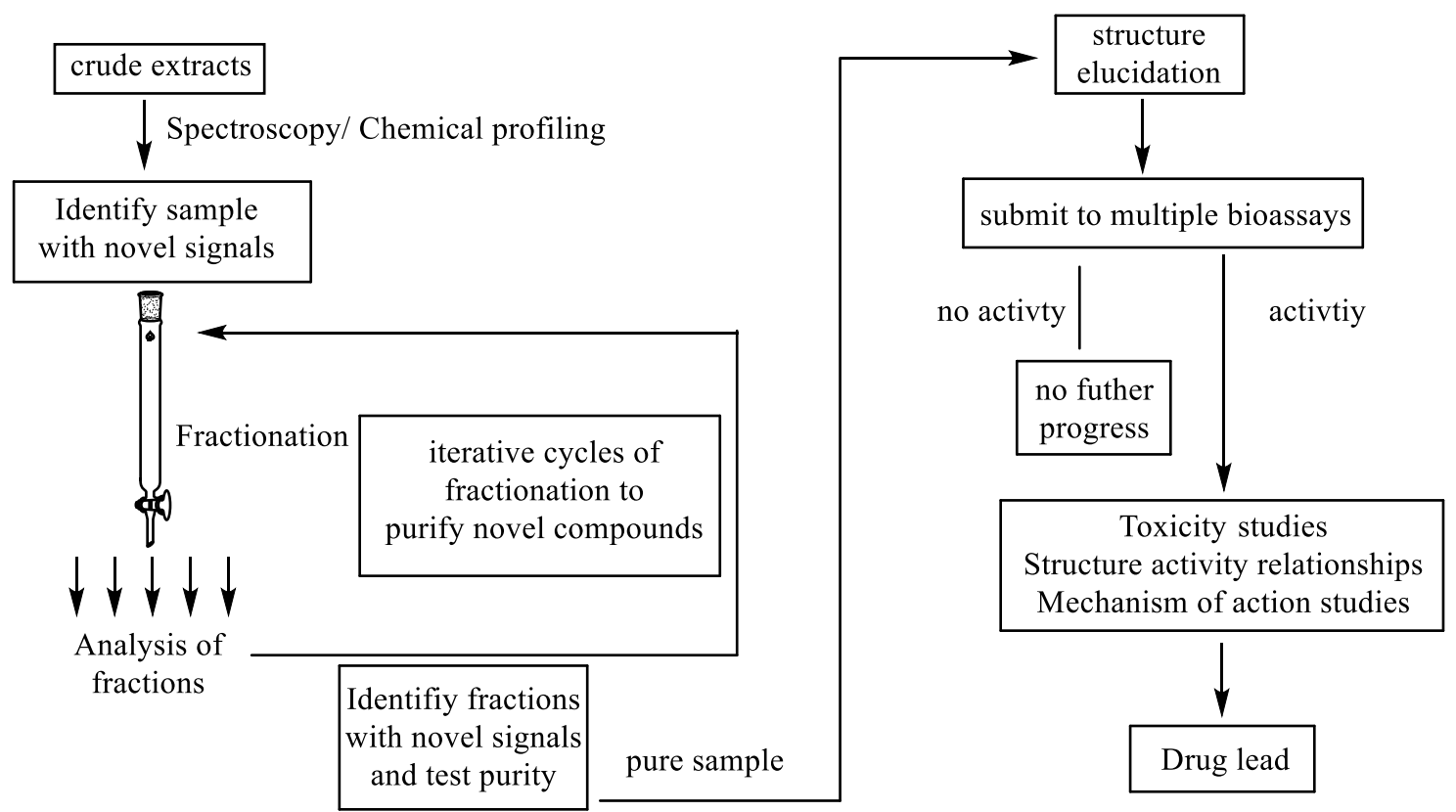

Figure 1.2 Structure-guided isolation.

The principles of structure-guided isolation of novel compounds.

\subsubsection{Structure-activity relationships and mechanism of action studies}

After isolation of a novel compound, the next stage of the drug discovery process involves structure-activity relationship (SAR) and mechanism of action (MOA) studies. While SAR studies are vital in pinpointing the pharmacophore responsible for bioactivity within a compound, MOA studies answer the important question of how a compound causes an observed activity.

SAR involves chemical modification of the lead compound to generate analogues that are then tested in the assay of interest. In addition to identifying functionalities crucial for bioactivity, it could also identify functional groups of the molecule that are open for modification in order to improve activity or other properties (such as solubility) when compared to the parent compound [113]. 
One approach for MOA studies involves attaching the drug of interest to sepharose beads and using affinity chromatography on cell extracts to purify proteins that bind to the protein-bound solid phase support [117]. Alternatively, genetic approaches can also be used with the underlying commonality of using or generating mutant strains. One approach involves genome sequencing of resistant mutants to identify mutations conferring drug resistance [118]. This is generally followed by validation assays, which can involve restoring normal functionality of the mutated gene in order to see a return in sensitivity to the compound of interest [51]. Another approach involves overexpressing the drug target to either purify protein to perform biochemical assays or to induce phenotypic effects on drug sensitivity [66]. As some compounds may have no specific target or may target an essential gene, no specific mutants will be detected and this is a limitation to this approach.

If the target is unknown a genome-wide approach can be employed, which involves screening of the compound of interest against mutant libraries in order to find mutants that interact with the drug. Following the identification of mutants, validation through dose response curves is performed, which allows for the top mutants to be chosen. These mutants are then sequenced to identify the specific gene that has been mutated. Once this information is inferred, a genetic network can then be established to identify which pathways may be perturbed by the compound of interest. This approach generates an immense amount of data which can then be used to carry out follow up validation and rescue assays to study the effects of each mutation.

\subsubsection{Saccharomyces cerevisiae and chemical genetics}

Saccharomyces cerevisiae (baker's yeast) is an important tool for mode of action studies by chemical genetics [119]. Yeast can exist in diploid or haploid states, and extensive genetic manipulation has led to generation of libraries of homozygous diploid deletion mutants of non-essential genes, heterozygous diploid knock-outs that include essential genes and haploid libraries for the two mating types of yeast [120]. Each knockout mutant has its gene replaced by a kanamycin resistance gene flanked by a 20 nucleotide long sequence unique for each mutant similar to barcodes [121]. Flanking these 'barcodes' are two lengths of nucleotides at the 5' and 3' ends. These nucleotide 
sequences are identical and allow for the use of just one set of primers to bind to and amplify the sequence in between [121].

These libraries have led to the development of multiple approaches for MOA studies, such as haploinsufficiency profiling (HIP), homozygous deletion profiling (HOP), synthetic lethality analysed by microarray (SLAM) and synthetic genetic array (SGA) [122].

HIP involves knocking out one of the two genes that code for a particular function within the yeast [123]. Having one less copy results in a decreased amount of the gene product, therefore strains are more sensitive to inhibitors that target that particular gene [123]. This approach is carried out in a pooled format where the mutants are grown in a liquid culture in either the presence or absence the drug, following which polymerase chain reaction amplification of the two different pools of mutants and microarray analysis of the oligomers allows for the identification of mutants that are most sensitive to the drug [124]. This approach allows inferences to be made on the actual drug target as the strains used in this approach are heterozygous knock outs and include essential genes.

HOP involves treating the library of mutants with drug followed by microarray analysis to identify an expression profile. These are compared to the expression profile with homozygous knockouts, which as a consequence are non-essential gene knock-outs. The closest matching deletion profiles allow for conclusions to be drawn as to which pathways the drug of interest is interacting with [125].

SLAM and SGA use haploid strains of yeast and work on the principle of synthetic lethality. This is the phenomenon where two mutations on their own have no effect on viability, however if the two mutations exist in the same cell then this results in cell death. In this way, profiles have been generated for libraries that have an additional "query" mutation introduced to identify synthetic lethal genetic interactions [122]. SLAM exploits the use of the unique tags by performing the experiment in a pooled format and analysing for the absence of drug exposed mutants using microarray analysis [126]. SGA monitors the growth of each mutant on agar and identifies growth defects in mutants exposed to drug [127]. Both approaches assume a drug can replace the role of a genetic mutation. Should this hold true, then synthetic lethal profiles observed for the drug exposed mutants should be possible to match to the profile of a query gene's 
interactions. Using this approach conclusions can be drawn on pathways the drug interacts with or perturbs.

\subsection{Research goals}

The aims of this research were to isolate novel anti-mycobacterial compounds from marine organisms. This would be achieved through:

Aim I: Detecting anti-mycobacterial activity from screens of New Zealand marine organism extract libraries.

Aim II: Purification and identification of anti-mycobacterial bioactives using bioassay-guided fractionation and spectroscopic structural characterization.

Aim III: Determination of the mechanism of action via studies of novel antiMycobacterium tuberculosis inhibitors. 


\section{Chapter 2 Materials and Methods}




\subsection{Media and culture conditions}

\subsubsection{LB broth}

LB broth (Duchefa Biochemie low salt) was made up as per instructions on the container, $20 \mathrm{~g}$ to $1 \mathrm{~L}$ of $\mathrm{dd}_{2} \mathrm{O}$, autoclaved at $121{ }^{\circ} \mathrm{C}$ for $30 \mathrm{~min}$ and stored at room temperature before use.

\subsubsection{Media supplements}

Additional antibiotics and supplements were added as required for certain strains of bacteria and have been listed when used in later sections.

Antibiotics were purchased from Sigma and were dissolved in an appropriate solvent or ${ }_{\mathrm{dd}} \mathrm{H}_{2} \mathrm{O}$ at desired concentrations and filtered through a $0.22 \mu \mathrm{m}$ syringe filter. Antibiotic stock solutions were aliquoted into microfuge tubes and stored at $-20^{\circ} \mathrm{C}$.

Isopropyl- $\beta$-D-thigalactopyranoside (IPTG) was made up with ${ }_{\mathrm{dd}} \mathrm{H}_{2} \mathrm{O}$ at $100 \mathrm{mg} / \mathrm{mL}$ filtered through a $0.22 \mu \mathrm{m}$ syringe filter and stored at $-20{ }^{\circ} \mathrm{C}$.

D-Arabinose and D-xylose were made up to a $100 \mathrm{mg} / \mathrm{mL}$ and a $10 \% \mathrm{w} / \mathrm{v}$ solution respectively in ${ }_{\mathrm{dd}} \mathrm{H}_{2} \mathrm{O}$, filtered through a $0.22 \mu \mathrm{m}$ syringe filter and stored at $-20{ }^{\circ} \mathrm{C}$ until use.

Glycerol was made up to $50 \%(\mathrm{v} / \mathrm{v})$ with $\mathrm{dd}_{\mathrm{d}} \mathrm{H}_{2} \mathrm{O}$, sterilised in an autoclave at $121{ }^{\circ} \mathrm{C}$ for $30 \mathrm{~min}$ and was added to media when required, prior to autoclaving.

Tween 80 was made up to $10 \%(\mathrm{v} / \mathrm{v})$ with ${ }_{\mathrm{dd}} \mathrm{H}_{2} \mathrm{O}$ and sterilised in an autoclave at $121{ }^{\circ} \mathrm{C}$ for $30 \mathrm{~min}$. This was added to media when required prior to autoclaving.

Sodium oleate was made by adding $12.5 \mathrm{~mL}$ of $2 \mathrm{M} \mathrm{NaOH}$ and $6.0 \mathrm{~mL}$ oleic acid to $481.5 \mathrm{~mL}$ of ${ }_{\mathrm{dd}} \mathrm{H}_{2} \mathrm{O}$. This solution was filtered through a $0.22 \mu \mathrm{m}$ millipore filter, stored at room temperature and the container was wrapped in aluminium foil.

OADC enrichment supplement for $7 \mathrm{H} 9$ media was made by adding $8.5 \mathrm{~g} \mathrm{NaCl}$ to 925 $\mathrm{mL}{ }_{\mathrm{dd}} \mathrm{H} 2 \mathrm{O}$ that was stirred until the salt has dissolved, $50 \mathrm{~g}$ of bovine serum albumin Fraction $\mathrm{V}$ was then added as well as $20 \mathrm{~g}$ of dextrose and $50 \mathrm{~mL}$ of a $1 \%$ sodium oleate solution. The $\mathrm{pH}$ was adjusted to 7.0 and this solution was filtered through a $0.22 \mu \mathrm{m}$ millipore filter and stored at $4{ }^{\circ} \mathrm{C}$. 


\subsubsection{LB broth for M. smegmatis}

LB broth (section 2.1.1) was prepared with additional supplements $0.1 \%(\mathrm{v} / \mathrm{v})$ Tween 80 and D-arabinose $100 \mu \mathrm{g} / \mathrm{mL}$ and autoclaved at $121{ }^{\circ} \mathrm{C}$ for $30 \mathrm{~min}$. After the broth had reached room temperature, kanamycin sulphate was added to achieve either a final $50 \mu \mathrm{g} / \mathrm{mL}$ or $20 \mu \mathrm{g} / \mathrm{mL}$ concentration. This was done in a sterile PC2 laminar flow hood following which media was stored at $4{ }^{\circ} \mathrm{C}$. When making media to culture electro-competent cells or carry out transformations, D-arabinose and antibiotics were omitted from media, which was stored at room temperature before use.

\subsubsection{H9 media}

Middlebrook 7H9 media was made as per instructions on the container (4.7 $\mathrm{g}$ per $900 \mathrm{~mL}$ ) and autoclaved at $121{ }^{\circ} \mathrm{C}$ for $30 \mathrm{~min}$. After the broth had cooled to room temperature it was supplemented with $10 \%$ (v/v) OADC (section 2.1.1.1). This was done in a sterile PC2 laminar flow hood following which media was stored at $4{ }^{\circ} \mathrm{C}$.

\subsubsection{Tissue culture media}

RPMI-1640 media was purchased from Invitrogen and supplemented with $0.22 \mu \mathrm{m}$ filtered $10 \%$ FCS and $1 \%$ of Pen/Strep (100 units/mL of penicillin and 100 units/mL of streptomycin). Handling of media was carried out in a sterile PC2 laminar flow hood and stored at $4{ }^{\circ} \mathrm{C}$ until use.

\subsection{Phosphate buffered saline}

Phosphate buffered saline (PBS) solution was made by adding $8 \mathrm{~g}$ of $\mathrm{NaCl}, 0.2 \mathrm{~g}$ of $\mathrm{KCl}$, $1.42 \mathrm{~g}$ of $\mathrm{Na}_{2} \mathrm{HPO}_{4}$ and $0.27 \mathrm{~g}$ of $\mathrm{KH}_{2} \mathrm{PO}_{4}$ to $900 \mathrm{~mL}$ of ${ }_{\mathrm{dd}} \mathrm{H}_{2} \mathrm{O}$. The $\mathrm{pH}$ of the solution was adjusted to 7.0 using $1 \mathrm{M}$ solutions of $\mathrm{HCl}$ and $\mathrm{NaOH}$, and the final volume made up to $1.0 \mathrm{~L}$ before autoclaving the solution at $121{ }^{\circ} \mathrm{C}$ for $30 \mathrm{~min}$. The solution was stored at room temperature.

\subsection{Staining procedures}

Cells were stained to observe the purity of culture using the methods describe below. Dyes and staining solutions were purchased from BD Biosciences. 


\subsubsection{Gram staining}

A $10 \mu \mathrm{L}$ aliquot of culture was smeared and air dried onto a slide. The slide was covered in Gram Crystal Violet solution for one minute and washed with water, following which the slide was covered in Stabilized Gram Iodine solution for one minute. The slide was then washed with water and decolourised using Gram Decolourizer for $10 \mathrm{sec}$. Finally Gram safranin was used to cover the slide for $30 \mathrm{sec}$ and washed off with water and air dried. Cover slips were placed on slides and slides observed under 100 x oil immersion using a light microscope.

\subsubsection{Acid fast staining}

A $10 \mu \mathrm{L}$ aliquot of culture was smeared and air died onto a slide. The slide was covered in TB Carbol Fuchsin KF solution for five minutes and washed with water, following which the slide was decolourised using TB Decolorizer solution for $10 \mathrm{sec}$. Finally TB Brilliant Green solution was used to counter stain the slide for $30 \mathrm{sec}$ and washed off with water and air dried. Cover slips were placed on slides and slides were observed under $100 \mathrm{x}$ oil immersion using a light microscope.

\subsubsection{Fluorescent microscopy}

Green Fluorescent Protein (GFP) expressing cells (5 $\mu \mathrm{L})$ were prepared on a slide and coverslip and observed under a $100 \mathrm{x}$ oil immersion using a fluorescein isothiocyanate (FITC) filter in an Olympus ${ }^{\circledR}$ Research Microscope AX70 Provis, equipped with an OlympusR DP70 Digital Microscope Camera.

\subsection{Transformation of mycobacteria}

\subsubsection{Plasmids}

Table 2.1 Plasmids used in this study

\begin{tabular}{lllc}
\hline Plasmid & Characteristics & Selection & Reference \\
\hline pLL192_hsp60 & Constant expression of GFP. & Strep 50 $\mu \mathrm{g} / \mathrm{mL}$, & {$[128]$} \\
pCG79 & Kan $50 \mu \mathrm{g} / \mathrm{mL}$ & \\
& $\begin{array}{l}\text { Contains the Tn611 transposon } \\
\text { shuttle vector and a temperature } \\
\text { sensitive mycobacterial origin of } \\
\text { replication }\end{array}$ & & \\
& & & \\
\hline
\end{tabular}




\subsubsection{Creating electro-competent Mycobacterium smegmatis}

Mycobacterium smegmatis, $\mathrm{mc}^{2} 155$ (ATCC700084); was the strain of mycobacteria used in this research and was sourced from Dr. Desmond Collins, AgResearch Limited, New Zealand. M. smegmatis was streaked out onto LB agar plates and grown for 3-5 days until colonies appeared. A loop full of colonies were then used to inoculate $10 \mathrm{~mL}$ of LB broth (section 2.1.1.2) and incubated at $37{ }^{\circ} \mathrm{C}$ in a shaking incubator at $200 \mathrm{rpm}$ overnight. This culture was then sub-inoculated into $100 \mathrm{~mL}$ of $\mathrm{LB}$ broth (section 2.1.1.2). Growth was measured once every $1.5 \mathrm{~h}$ using optical density (OD) measured at $600 \mathrm{~nm}$. Once an $\mathrm{OD}_{600}$ between 0.5-1.0 absorbance units (AU) was achieved, cells were incubated on ice for $20 \mathrm{~min}$ and centrifuged at $2700 \mathrm{x} \mathrm{g}$ at $4{ }^{\circ} \mathrm{C}$ for $15 \mathrm{~min}$. After discarding the supernatant, cells were washed and pelleted a further two times with 50 $\mathrm{mL}$ and $20 \mathrm{~mL}$ of ice cold $10 \%$ glycerol with a $15 \mathrm{~min}$ incubation on ice in between. After pelleting cells a third time, cells were re-suspended in $4 \mathrm{~mL}$ of ice cold $10 \%$ glycerol and $200 \mu \mathrm{L}$ aliquots of the competent cells were stored in $1.5 \mathrm{~mL}$ tubes at $-80{ }^{\circ} \mathrm{C}$.

\subsubsection{Transformation of Mycobacterium smegmatis}

To electroporation cuvettes $200 \mu \mathrm{L}$ of electro-competent (e $\mathrm{e}^{-}$) cells (section 2.4.2) and $200 \mu \mathrm{L}$ of $10 \%$ glycerol was added. To the $\mathrm{e}^{-}$cells $5 \mu \mathrm{L}$ of either the plasmid pLL192_hsp60 or pCG79 was added. As a negative control $5 \mu \mathrm{L}$ of MQ $\mathrm{H}_{2} \mathrm{O}$ was added. Cells were electroporated at the following settings, voltage: $2500 \mathrm{~V}$, capacitance: $25 \mathrm{C}$, resistance: $1000 \Omega$, cuvette width: $2 \mathrm{~mm}$. Following electroporation a $600 \mu \mathrm{L}$ aliquot of LB broth without antibiotics (section 2.1.1.2) was added to the cuvettes immediately and cells were transferred into sterile $1.5 \mathrm{~mL}$ centrifuge tubes. Cells were allowed to recover overnight at $37{ }^{\circ} \mathrm{C}$ at $200 \mathrm{rpm}$. The following day cells were plated out onto $\mathrm{LB}$ agar with appropriate antibiotics (Table 2.1). Appearance of colonies only for the mycobacteria carrying plasmids following 2-3 days of incubation indicated a successful electroporation. Colonies were then inoculated in LB broth for mycobacteria (section 2.1.1.2) with appropriate antibiotic (Table 2.1). The overnight cultures were then tested for purity using acid fast staining and fluorescence using a fluorescent microscope (section 2.3.2 \& 2.3.3). Freezer stocks of cultures were then made by adding $600 \mu \mathrm{L}$ of cells to $400 \mu \mathrm{L}$ of $50 \%$ glycerol, following which cells were stored at $-80{ }^{\circ} \mathrm{C}$. 


\subsection{Bacteria and mammalian cell line culturing}

Table 2.2 Mycobacteria used in this study

\begin{tabular}{ll}
\hline Strain & Use \\
\hline M. smegmatis & \\
pLL192_hsp60 & Library screening and dose response assays, \\
pCG79 & Transposon mutant library screening \\
M. tuberculosis & \\
H37Ra ATCC25177 & Dose response assays \\
\hline
\end{tabular}

\subsubsection{Culturing of Mycobacterium smegmatis}

Freezer stocks of M. smegmatis carrying either the pLL192_hsp60 or pCG79 plasmid were streaked out on LB agar with an appropriate drug and incubated for $48 \mathrm{~h}$ at $37^{\circ} \mathrm{C}$. Following the appearance of colonies a $5 \mu \mathrm{L}$ inoculating loop was used to scoop bacteria into $10 \mathrm{~mL}$ of $\mathrm{LB}$ broth (section 2.1.1.2). After growth incubation for $24 \mathrm{~h}$ these cultures were checked for purity using either acid fast staining or fluorescence (section 2.3.2 \& 2.3.3) before being used for bioassay.

\subsubsection{Mycobacterium tuberculosis H37Ra}

M. tuberculosis $\mathrm{H} 37 \mathrm{Ra}$ ATCC25177 was grown on $7 \mathrm{H} 10$ plates for $3-4$ weeks at $37{ }^{\circ} \mathrm{C}$, after which colonies were picked and cultured in 7H9 media (section 2.1.2). After approximately 14 days, cells from these cultures were checked for purity using acid fast staining (section 2.3.2) following which they were used for bioassay. 


\subsubsection{Staphylococcus aureus, Escherichia coli and Pseudomonas aeruginosa.}

Table 2.3 Fast growing bacterial strains

\begin{tabular}{|c|c|c|c|}
\hline Strain & Characteristic & $\begin{array}{l}\text { Culture } \\
\text { conditions }\end{array}$ & Use \\
\hline \multicolumn{4}{|l|}{ S. aureus } \\
\hline ATCC25923 & $\begin{array}{l}\text { Clinical isolate, no resistance } \\
\text { marker }\end{array}$ & LB & $\begin{array}{l}\text { Dose response } \\
\text { assays }\end{array}$ \\
\hline Newman & $\begin{array}{l}\text { Expresses GFP, erythromycin } \\
(\text { Em) resistance }\end{array}$ & $\begin{array}{l}\mathrm{LB}, 0.5 \% \\
(\mathrm{w} / \mathrm{v}) \text { xylose, } \\
\mathrm{Em} \\
50 \mu \mathrm{g} / \mathrm{mL}\end{array}$ & $\begin{array}{l}\text { Library } \\
\text { screening }\end{array}$ \\
\hline \multicolumn{4}{|l|}{ E. coli } \\
\hline ATCC25922 & $\begin{array}{l}\text { Clinical isolate, no resistance } \\
\text { marker }\end{array}$ & LB & $\begin{array}{l}\text { Dose response } \\
\text { assays }\end{array}$ \\
\hline DH5 $\alpha /$ pOT11 & $\begin{array}{l}\text { IPTG induced expression of } \\
\text { GFP, chloramphenicol (Chl) } \\
\text { resistance }\end{array}$ & $\begin{array}{l}\mathrm{LB}, \mathrm{Chl} 25 \\
\mu \mathrm{g} / \mathrm{mL}, \mathrm{IPTG} \\
(100 \mu \mathrm{g} / \mathrm{mL})\end{array}$ & $\begin{array}{l}\text { Library } \\
\text { screening }\end{array}$ \\
\hline \multicolumn{4}{|l|}{ P. aeruginosa } \\
\hline PAO1 & $\begin{array}{l}\text { Opportunistic human pathogen, } \\
\text { no resistance marker }\end{array}$ & LB & $\begin{array}{l}\text { Dose response } \\
\text { assays }\end{array}$ \\
\hline
\end{tabular}

Freezer stocks of cultures were streaked onto LB agar (Duchefa Biochemie) with appropriate antibiotics (Table 2.3). After incubating the plates overnight a $5 \mu \mathrm{L}$ inoculating loop was used to scoop colonies into $10 \mathrm{~mL}$ of LB broth with appropriate antibiotics and supplements (Table 2.3). Cultures were incubated overnight in a $37{ }^{\circ} \mathrm{C}$ shaking incubator at $200 \mathrm{rpm}$. Gram staining was used to check the purity of the cultures (section 2.3.1) which were used to for bioassay.

\subsubsection{HL-60 cells}

The HL-60 cells used in this research were supplied by Dr. John Miller, VUW; and were sourced from Dr. Graeme Findlay, University of Auckland. HL-60 cells were thawed out from frozen stocks and transferred to $15 \mathrm{~mL}$ tubes containing $9 \mathrm{~mL}$ of RPMI-1640 supplemented media (section 2.1.3). Cells were pelleted at $300 \mathrm{x} \mathrm{g}$ for 5 min and the supernatant was discarded. The pellet was then resuspended in $10 \mathrm{~mL}$ of media and transferred to a $25 \mathrm{~cm}^{3}$ flask. Cells were incubated at $37{ }^{\circ} \mathrm{C}$ and $5 \% \mathrm{CO}_{2}$ and after reaching approximately $80 \%$ confluence cells were pelleted at $300 \mathrm{x} \mathrm{g}$. Cells were 
then resuspended in $5 \mathrm{~mL}$ of media and $10 \mu \mathrm{L}$ of cells were added to $10 \mu \mathrm{L}$ of trypan blue to count the number of viable cells using a haemocytometer.

\subsubsection{HEK cells}

Human embryonic kidney-293 (HEK) cells were supplied by Dr. Bronwyn Kivell VUW; and were sourced from (ATCC, Manassas, VA, USA). HEK cells were thawed as stated above and cultured in $10 \mathrm{~mL}$ of media (section 2.1 .3 ) until they had reached $\sim 80 \%$ confluence. Following this, media was discarded from the flask and cells were trypsinised with $1 \mathrm{~mL}$ of trypsin for 2-3 min, media was then added to the flask to deactivate the trypsin. After centrifuging cells for $5 \mathrm{~min}$ at $300 \mathrm{x} \mathrm{g}$, the media was decanted out and the cells were resuspended in $5 \mathrm{~mL}$ of media. Cell counts were performed as stated above (section 2.5.4).

Freshly cultured HL-60 and HEK cells were used to set up assays described in this research. This was done to avoid any issues that may arise from using cells that have undergone several passages.

\subsubsection{Marine extract libraries}

The NIWA library was a four plate library donated by Drs Vicky Webb and Sally Anderson. The library was generated as a nutraceutical library, which contained extracts of vertebrate as well as invertebrates collected from New Zealand waters, and did not include algae. Marine organisms were dissected and extracts were generated of each organ. The library plates contained either water, ethanol or ethyacetate extracts and comprised unknown masses per well.

The VUW MNP library was generated in Dr. Rob Keyzers laboratory using extracts of invertebrates, which also included algae. Samples were collected by Dr. Peter Northcote from New Zealand coastal waters and were extracted in methanol. Extracts were dried down and redissolved to generate solutions of known concentrations after which aliquots were transferred to a 96 well plate to generate a library plate of $50 \mu \mathrm{g}$ per well. The locations from where samples were collected are on record, and voucher samples were generated from each sample used to generate the library. 


\subsection{Assay protocols}

\subsubsection{Assay measurements}

Fluorescence and OD measurements of assays were measured using a PerkinElmer EnSpire, 2300 multilable reader. OD was measured at $600 \mathrm{~nm}$ taking an average of 16 points per well $(4 \times 4$ circle) for mycobacteria, and a single point per well for all other cells. GFP fluorescence was measured at excitation (ex): $488 \mathrm{~nm}$ and emission (em): $597 \mathrm{~nm}$ taking an average of 16 points per well (4x4 circle) for mycobacteria, and a single point per well for all other cells. GFP was used as a measure of bacterial viability, as only live cells would be expected to express GFP and cells inhibited in growth will not.

After GFP and OD was measured, a $30 \mu \mathrm{L}$ aliquot of a $0.02 \%(w / v)$ solution of resazurin were added to each well of the assay plates. After $2 \mathrm{~h}$ for $M$. smegmatis, or 20 min for S. aureus, E. coli, and P. aeruginosa, fluorescence of resazurin was measured at ex: $530 \mathrm{~nm}$ and em: $590 \mathrm{~nm}$ taking a single point measurement per well. For M. tuberculosis H37Ra plates were incubated for $24 \mathrm{~h}$ before measuring resazurin fluorescence. This additional method to measure growth was also used to add further support for measuring inhibition. Resazurin is a dye that is reduced by actively metabolizing bacteria and in the process goes from a non-fluorescent blue to a fluorescent pink, which can be exploited as a measure of bacterial viability [130].

\subsubsection{Screening of marine extract libraries}

Marine extract libraries were resuspended in DMSO and aliquoted in 96-well plates with $50 \mu \mathrm{L}$ of broth with a maximum final concentration of $2.5 \%(\mathrm{v} / \mathrm{v})$ DMSO. To this $50 \mu \mathrm{L}$ of either M. smegmatis, S. aureus, or E. coli were added to wells to achieve a final $\mathrm{OD}_{600}$ of 0.01 AU. Screening was carried out such that 80 extracts were screened per 96-well plate. In the wells of column 1, $100 \mu \mathrm{L}$ MQ $\mathrm{H}_{2} \mathrm{O}$ was added to maintain a humid environment and prevent evaporation and column 12 comprised of a set of controls in duplicate: media alone, media+cells, media+cells and solvent, media+cells and a positive control (ethambutol $250 \mu \mathrm{M}$ or tetracycline $250 \mu \mathrm{M}$ ) (Figure 2.1). Plates were sealed using breathable SealPlate ${ }^{\circledR}$ plate seals (Interlab) and incubated for $96 \mathrm{~h}$ at $37^{\circ} \mathrm{C}$ in a shaking incubator at $200 \mathrm{rpm}$. 


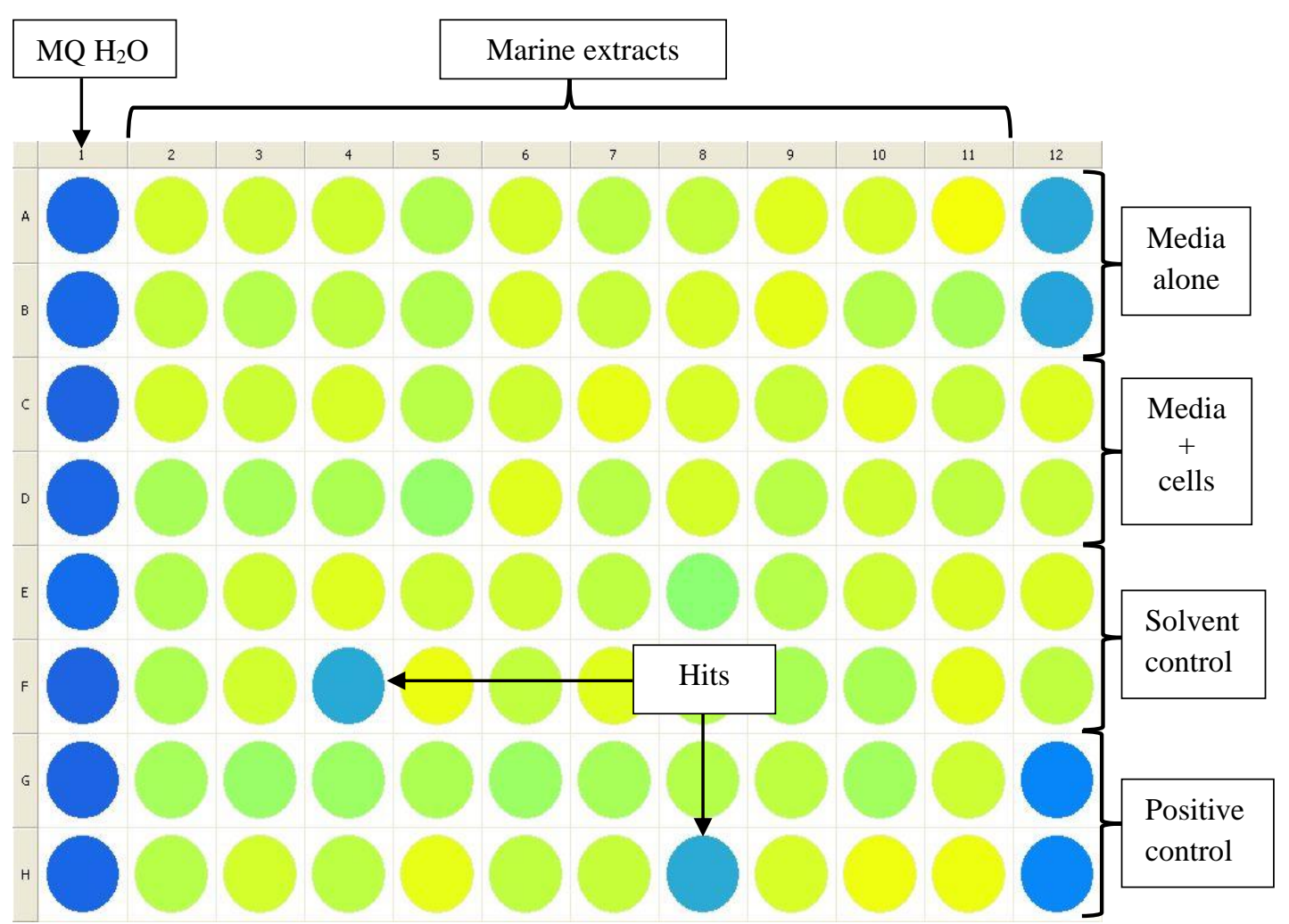

Figure 2.1 General set up of a 96-well plate for screening marine extract libraries

Following the assay, plates were measured for GFP and resazurin fluorescence as described in section 2.6.1.

\subsubsection{Bacteriostatic assay}

Samples were serial diluted two fold in appropriate media in 96-well plates to test the range of concentrations at which they were active. To the outer 36 wells of a 96-well plate, $100 \mu \mathrm{L}$ of MQ was added and to the inner 60 wells except those of column two, $50 \mu \mathrm{L}$ of media was added. The wells of column two were made up to a final volume of $100 \mu \mathrm{L}$ by adding the appropriate amounts of media and sample to give an initial two fold higher starting concentration of extract. Following this $50 \mu \mathrm{L}$ of the mixture was aspirated from the wells of column two and pipetted to column three, this step was repeated until the wells of column 11 and the final $50 \mu \mathrm{L}$ was discarded. To these plates, $50 \mu \mathrm{L}$ of cells were added to achieve a final $\mathrm{OD}_{600}$ of $0.01 \mathrm{AU}$. Plates were sealed and incubated for $96 \mathrm{~h}$ at $37{ }^{\circ} \mathrm{C}$ in a shaking incubator at $220 \mathrm{rpm}$. 
As a positive control for inhibition, every assay had either rifampicin for M. smegmatis or tetracycline for S. aureus, E. coli, and P. aeruginosa, starting at $250 \mu \mathrm{M}$. Negative controls included media alone and cells grown without the presence of any inhibitors. Solvent controls were also included. Plates were measured for GFP, OD and resazurin fluorescence as described in section 2.6.1.

\subsubsection{Bacteriostatic assay set up in M. tuberculosis H37Ra}

Sample dilutions and plate set up was identical to section 2.6.3, using 7H9 media with supplements (section 2.1.2). This was followed by addition of cells to plates to achieve a final $\mathrm{OD}_{600}$ of $0.005 \mathrm{AU}$, after which plates are sealed and incubated for 7 days at $37{ }^{\circ} \mathrm{C}$ at $200 \mathrm{rpm}$. Streptomycin starting at $25 \mu \mathrm{M}$ was used a positive control. Following incubation, plates are unsealed in a laminar flow hood and $30 \mu \mathrm{L}$ of a $0.02 \%$ $(\mathrm{w} / \mathrm{v})$ of resazurin was added to each well and plates were resealed and incubated a further $24 \mathrm{~h}$ at $37{ }^{\circ} \mathrm{C}$ in a stationary incubator. Resazurin fluorescence was measured as described in section 2.6.1.

\subsubsection{Stationary phase assay protocol in M. tuberculosis H37Ra}

Following the method described by Bassett et al. cultures of M. tuberculosis H37Ra were grown for 30 days, pelleted for $10 \mathrm{~min}$ at $2700 \mathrm{x}$ g and resuspended in a volume of sterile PBS (section 2.2) to a final $\mathrm{OD}_{600}$ of 1.0 AU. Plate set up was identical to section 2.6.3 using sterile PBS. To the plates, $50 \mu \mathrm{L}$ of the diluted cells were added to achieve a final $\mathrm{OD}_{600}$ of $0.5 \mathrm{AU}$. Plates were sealed and incubated for $96 \mathrm{~h}$ at $37{ }^{\circ} \mathrm{C}$ at $200 \mathrm{rpm}$ after which $30 \mu \mathrm{L}$ of a $0.02 \%(w / v)$ of resazurin was added to each well and plates were resealed [131]. Resazurin fluorescence was measured as described in section 2.6.1. 


\subsubsection{Statistics for library screening}

Z-factors were calculated for each assay and used as a measure of the validity of the screens. The range of the Z-factor can be between $0-1$, where 0 indicates an invalid assay due to overlap between positive and negative controls and 1 indicates a perfect assay. Values of 0.5 or above are considered acceptable.

$$
Z=1-\frac{3\left(\sigma_{\text {positive }}+\sigma_{\text {negative }}\right)}{\left|\mu_{\text {positive }}-\mu_{\text {negative }}\right|}
$$

Figure 2.2 Z- factor calculation

$\mu$ represents the mean of the positive or negative controls and $\sigma$ is the standard deviation of the positive and negative controls.

\subsubsection{Reporting inhibitory values}

Where possible, $\mathrm{IC}_{50}$ values that have been reported are based on calculated values after using the stats software package GraphPad Prism V. 5.00 for Windows. As sigmoidal curves were not possible in all cases of dose response assays, $\mathrm{MIC}_{90}$ values have been listed. $\mathrm{MIC}_{90}$ values reported throughout this thesis are based on the lowest concentration at which $90 \%$ or greater growth inhibition is observed directly in all wells of the biological replicates. Minimum inhibitory concentration (MIC) values in this thesis are the concentration at which $\geq 98 \%$ inhibition is observed in all wells of the biological replicate.

\subsubsection{HL-60 MTT assay}

Samples were serial diluted two-fold in RPMI media using the same method as described in section 2.6 .3 , following which $50 \mu \mathrm{L}$ of cells at $2 \times 10^{5}$ cells $/ \mathrm{mL}$ were then added to plates to achieve a final cell number per well of $1 \times 10^{5}$ cells $/ \mathrm{mL}$. Plates were incubated for $48 \mathrm{~h}$ at $37{ }^{\circ} \mathrm{C}$ and $5 \% \mathrm{CO}_{2}$. Internal controls included solvent controls, media with cells and media alone. After $48 \mathrm{~h}, 20 \mu \mathrm{L}$ of MTT (5 mg/mL in PBS) was added to each well and plates were wrapped with cling wrap and incubated for $2 \mathrm{~h}$. Following incubation with MTT, $100 \mu \mathrm{L}$ of solubiliser (45\% DMF, 10\% SDS at pH 4.5) 
was added to all wells and plates were incubated overnight. The next day, OD of plates was measured at $570 \mathrm{~nm}$.

\subsubsection{HEK MTT assay}

Cells were diluted to $1 \times 10^{5}$ cells $/ \mathrm{mL} ; 100 \mu \mathrm{L}$ of cells was then added to the inner 60 wells of a 96-well plate, and plates were then incubated for $24 \mathrm{~h}$. The following day, $100 \mu \mathrm{L}$ of media was taken out of each initial well and the volume of that well was made up to $200 \mu \mathrm{L}$ with the appropriate amount of desired drug. From this initial well, $100 \mu \mathrm{L}$ was taken out and added to the next well, after thorough pipetting, $100 \mu \mathrm{l}$ was taken out from this second well and the process was repeated to achieve the desired number of serial dilutions. Internal controls were incorporated as stated in the protocol of HL-60 cells in section 2.6.6. Plates were incubated for $72 \mathrm{~h}$ at $37{ }^{\circ} \mathrm{C}$ and $5 \% \mathrm{CO}_{2}$. After incubation, MTT and solubiliser was added to plates as stated above in section 2.6.6.

\subsection{Chemistry protocols}

Reversed-phase column chromatography was performed using Supelco Diaion ${ }^{\circledR}$ HP20 and HP20ss poly(styrene-divinylbenzene) (PSDVB) resins. Normal-phase column chromatography was performed using Supelco Discovery ${ }^{\circledR}$ DSC-DIOL functionalised silica: 3-(2,3-dihydroxy-propoxy)-propyl-silica (Diol).

To perform HPLC two systems were used, a Rainin Dynamax SD-200 solvent delivery system with $25 \mathrm{~mL}$ pump heads and a Varian Prostar 335 photodiode array detector for UV/Vis detection. The second HPLC system was an Agilent Technologies 1260 Infinity HPLC equipped with a quaternary pump, a thermostated column compartment and diode array detector (DAD). Following the DAD, a Quicksplit ${ }^{\mathrm{TM}}$ flow splitter directs $5 \%$ of the flow to an Agilent 380-evaporative light scattering detector (ELSD) with $95 \%$ of the flow directed towards collection.

The columns used in this research were reversed-phase, either a C18 analytical (Phenomenex Prodigy, 4.6 x $250 \mathrm{~mm}, 5 \mu \mathrm{m}$ particle size) or semi-preparative (Phenomenex Prodigy, 10.0 x $250 \mathrm{~mm}, 10 \mu \mathrm{m}$ particle size) columns. 
HPLC-grade solvents were used for all work (purchased from Fisher Scientific) except for hexanes and dichloromethane, which were Optima ${ }^{\circledR}$ grade. Water was distilled prior to use and for HPLC the water was further filtered through a membrane with a pore size of $0.45 \mu \mathrm{m}$ before use. The solvent compositions used are all reported as the $\% \mathrm{v} / \mathrm{v}$. TLC analyses were performed using Machery-Nagel Polygram ${ }^{\circledR}$ Sil G/UV 254 plates and visualised under UV light ( $254 \mathrm{~nm}$ and $350 \mathrm{~nm}$ ). TLC plates were then visualised by dipping in $5 \%$ conc. $\mathrm{H}_{2} \mathrm{SO}_{4}$ in methanol (v/v) followed by $0.1 \%$ vanillin in ethanol $(\mathrm{w} / \mathrm{v})$ and heated for analysis.

NMR spectra were obtained using a Varian DirectDrive spectrometer equipped with a triple resonance $\mathrm{HCN}$ cryogenic probe operating at $600 \mathrm{MHz}$ for ${ }^{1} \mathrm{H}$ and $150 \mathrm{MHz}$ for ${ }^{13} \mathrm{C}$ nuclei. ${ }^{19} \mathrm{~F}$ spectra were acquired using a Varian Inova $300 \mathrm{MHz}$ NMR at $280 \mathrm{MHz}$. The chemical shifts of ${ }^{1} \mathrm{H}$ and ${ }^{13} \mathrm{C}$ spectra were referenced to the residual solvent peaks $\left(\mathrm{CDCl}_{3}: \delta_{\mathrm{C}} 77.0, \delta_{\mathrm{H}} 7.26 ; \mathrm{CD}_{3} \mathrm{OD}: \delta_{\mathrm{C}} 49.0, \delta_{\mathrm{H}} 3.31 ; \mathrm{d}_{6} \mathrm{DMSO}: \delta_{\mathrm{C}} 39.5, \delta_{\mathrm{H}} 2.50\right)$. All NMR solvents were purchased from Aldrich with the exception of deuterated methanol, which was purchased from Cambridge Isotope Laboratories Inc.

NMR quantifications of masses less than $1 \mathrm{mg}$ were performed with an internal nitromethane $\left(\mathrm{CH}_{3} \mathrm{NO}_{2}\right)$ standard and using the acquisition parameters described by West [132].

HRESIMS results were obtained from an Agilent 6530 Q-TOF mass spectrometer equipped with an Agilent 1260 HPLC for solvent delivery utilising a JetStream ${ }^{\mathrm{TM}}$ electrospray ionisation source in positive and negative ion modes. Water and acetonitrile $(\mathrm{MeCN})$ solvents were both made up as $5 \mathrm{mM}$ solutions with ammonium formate.

For sponge identification, the organic matter was dissolved by immersing a small piece of sponge $\left(1 \mathrm{~cm}^{3}\right)$ in a boiling tube and adding concentrated $\mathrm{HNO}_{3}$ to cover the sample. Spicules that remained after this were then washed by suspending them in water and then centrifuging them to allow the water to be decanted. This process was repeated twice and the third time a $\mathrm{MeOH}$ wash was carried out. The spicules were then 
visualised using an Olympus ${ }^{\circledR}$ Research Microscope AX70 Provis equipped with an OlympusR DP70 Digital Microscope Camera and these images were compared with descriptions in the literature to classify the sponge.

\subsection{General NMR data acquisition protocols}

${ }^{1} \mathbf{H}$ :

number of scans 64

d1 relaxation delay $0.01 \mathrm{~s}$

Acquisition time $6.08 \mathrm{~s}$

Spectral width $0.0 \mathrm{ppm}-9.0 \mathrm{ppm}$

${ }^{13} \mathrm{C}$ :

number of scans 19776

d1 relaxation delay $0.01 \mathrm{~s}$

Acquisition time $1.73 \mathrm{~s}$

Spectral width $-10.0-230.0 \mathrm{ppm}$

${ }^{19} \mathbf{F}$ :

number of scans 52

d1 relaxation delay $1.00 \mathrm{~s}$

Acquisition time $1.00 \mathrm{~s}$

Spectral width $-200.0-30.0$ ppm

COSY: $\quad$ number of scans 1

number of increments 512

d1 relaxation delay $2.0 \mathrm{~s}$

Acquisition time $0.25 \mathrm{~s}$

Spectral width $0.0 \mathrm{ppm}-9.0 \mathrm{ppm}$ 
HSQC:

HMBC:

$1 D$ NOE:

1D TOCSY:

\section{.}




\subsection{Column chromatography}

\subsubsection{Cyclic loading}

To carry out cyclic loading, biomass samples were cut into approximately $3 \mathrm{~cm}^{3}$ pieces and allowed to steep in methanol $(\mathrm{MeOH})$ overnight. The $\mathrm{MeOH}$ from the first steep was decanted into a container and fresh $\mathrm{MeOH}$ was poured onto the sample which was allowed to steep again overnight. Column beds of HP20 (reversed-phase polystyrenedivinylbenzene copolymer) were set up based on the principle that $1 \mathrm{~kg}$ of sponge has $8 \mathrm{~g}$ of extractable compounds, and approximately $25 \mathrm{mg}$ of extractable compounds can bind onto $1 \mathrm{~mL}$ of HP20. Extracts were filtered through Büchner funnels with celite and filter paper, and extract was passed through the column at a flow rate of $4 \mathrm{~mL} / \mathrm{min}$. Following this, a two-fold dilution of the eluent was carried out with $\mathrm{H}_{2} \mathrm{O}$, this was then passed through the column again. Dilution of eluent was carried out by adding small volumes of $\mathrm{H}_{2} \mathrm{O}$ at a time, with care taken to avoid unwanted precipitation. If precipitation occurred, the solution was passed through the column again, before adding the rest of the volume of $\mathrm{H}_{2} \mathrm{O}$. These cycles of dilution and passing the solution through the column were repeated until a final $\mathrm{MeOH}$ concentration of $12.5 \%$ was passed through the column. Columns were eluted with 20\%, 40\%, 60\%, $80 \%$ and $100 \%$ mixtures of acetone in water. The $100 \%$ acetone fraction was evaporated under reduced pressure, while the other fractions were subject to backloading. 


\subsubsection{Backloading}

Backloading was employed to avoid issues with bumping and long evaporating times when drying down solvent mixtures that contain large volumes of water. HP20 acetone fractions were diluted two fold with $\mathrm{dd}_{\mathrm{d}} \mathrm{H}_{2} \mathrm{O}$ before passing them through a smaller HP20 column. Columns were eluted with three column volumes of $\mathrm{MeOH}$ and acetone, which was collected in a round bottom flask and evaporated under reduced pressure. The mass in the flask was dissolved in a small amount of a solvent mix of 4:1 MeOH: dichloromethane (DCM), which was then transferred to a pre-weighed glass sample vial. The vial was then evaporated in vacuo to obtain the absolute dry weight.

\subsubsection{HP20ss chromatography}

Columns were set up using the same principle for HP20 of $25 \mathrm{mg}$ of extractable material binding to $1 \mathrm{~mL}$ of resin, therefore for example for $100 \mathrm{mg}$ of a sample that needed to be fractionated, $4 \mathrm{~mL}$ of beads was used. This 'loaded HP20ss' was then transferred on the top of a larger column bed of HP20ss which was approximately three times the volume of the loaded HP20ss. Columns were eluted with appropriate solvent mixtures as described in the relevant methods sections at a flow rate of $1.5 \mathrm{~mL} / \mathrm{min}$.

\subsubsection{DIOL column chromatography}

Columns of the normal phase DIOL stationary phase (2,3-dihydroxy-propoxypropylderivatised silica gel.), were set up similarly to HP20ss columns, however the loading capacity estimate used was $10 \mathrm{mg}$ of compounds bind onto $1 \mathrm{~mL}$ of material. Columns were eluted with appropriate solvent mixtures as described in the relevant methods sections at a flow rate of $1.5 \mathrm{~mL} / \mathrm{min}$.

\subsubsection{LH20 chromatography}

For all LH20 size exclusion columns run, $100 \mathrm{~mL}$ of resin were soaked in appropriate running solvents overnight before setting up the column. Flow rates for all columns were maintained at approximately $0.2 \mathrm{~mL} / \mathrm{min}$. Fractions were collected in $10 \mathrm{~mL}$ test tubes using an automatic fraction collector. 


\section{Chapter 3 Screening and validations}




\subsection{Background}

In the preliminary research described in this chapter, libraries of crude marine extracts were screened to identify potential new sources of antitubercular activity. Two marine extract libraries were screened in 96-well plate format. The first was kindly donated by the National Institute of Water and Atmospheric Research (NIWA), with the second generated at VUW. For the NIWA library, the sample mass per well was unknown and varied between extracts. Validation as well as furthering of "hit" extracts with observed bioactivity depended on re-collection and re-extraction of the marine specimens. In contrast, the VUW marine natural product (MNP) library was generated in house with a constant mass per well across all crude extracts. Furthermore, biological samples were readily available should any be identified as hits.

As stated earlier, drug resistance in TB poses a serious health problem. In addition to this, general bacterial resistance to antibiotics pose a problem that must be addressed. A recent report by the Centers for Disease Control and Prevention estimated approximately 2 million people are infected with drug resistant bacteria leading to 23,000 deaths in America [133]. In NZ the rate of methicillin resistant S. aureus is 26.1 per 100 000, and 195.7 per 100000 for extended-spectrum $\beta$-lactamase producing Enterobacteriaceae in 2012 [134, 135]. In order to address drug resistance there is a need to identify new more effective antibiotics. This section highlights current problems with screening natural products as well as some approaches used to identify novel antibiotics.

\subsubsection{Current issues when screening for new antibiotics}

High-throughput screening (HTS) of natural products for antibiotics is not the most actively pursued research avenue by pharmaceutical companies [115]. There are certain aspects associated with screening of natural extracts for antibiotic activity that deter pharmaceutical companies from this field of research. First, accessibility to the samples proves a major hurdle, as it is often restricted due to intellectual property concerns of the governments involved $[114,136]$. Increased extinction of species that are sources of 
structural novelty due to human activities adds further restraints in sourcing material for drug discovery [137]. Drug companies need reliable access to the natural resource as well as patent control of any potential drug, to ensure ownership for the product upon which they have invested time and resources. In addition, seasonal variation and environmental changes are known to affect the production of metabolites of interest; this majorly affects reproducibility of natural product isolation during scale-up processes [138].

Provided such obstacles are overcome, the complexities of natural products chemistry are still left to be considered. For example, in a crude fraction it is possible that bioactivity may be due to a mixture of two or more compounds [114]. Following a rigorous purification process, the bioactive metabolite could be present in very low amounts, or be unstable. The presence of multiple stereogenic centres complicates matters even more, not only for structural elucidation but in the downstream process of identifying an economically viable synthetic route [114]. In such cases, structural elucidation of the metabolite can be even more complex, requiring uncommon NMR experiments or chemical derivatisation.

Re-isolation is another common issue encountered when screening natural products, where HTS that focus on activity alone may result in the re-isolation of so-called "promiscuous" known compounds, making the investigation redundant. For example, streptomycin is produced by $\sim 1 \%$ of all actinomycetes, subsequently there is a high chance that projects focusing on soil bacteria will lead to the isolation of this wellknown compound [139]. Repeat isolations of known compounds is a waste of both time and resources, a further disincentive for pharmaceutical companies to invest in this temperamental field [71].

The final test for a potential drug is how it performs in clinical trials. In total, the development of a new drug can take up to 15 years and cost up to a billion dollars [113, 140]. The high standards of regulatory authorities result in a high attrition rate during clinical trials; this means a vast amount of time and resources are devoted to drug candidates that may eventually fall out of the development pipeline [141].

Further reasons behind the waning interest of large pharmaceutical companies in antibacterial research is that there is a better return on investment in other disease research areas [115]. Antibiotics are taken for a short period of time and cure the disease, 
however treating chronic disease provide a better return as patients have to consume the medication for the remainder of their life $[142,143]$. Furthermore the expiry of patents for many drugs means that big Pharma have to compete with generic manufacturers [144]. For these reasons antibacterial research is no longer in vogue with Pharma, resulting in a decrease of commercially available, structurally unique antibiotics.

It is mainly due to cost cutting that recent screens have tended to focus on libraries comprising known chemical scaffolds, which has resulted is a lack of structurally diverse antibiotics $[143,145]$. To avoid resistance, combinatorial synthesis provides a powerful tool, by modifying a parent compound to introduce variation and improve activity [146]. However, the fact remains that it is still the same parent scaffold that is being used and there are certain limits as to how many modifications can be made to each compound [143]. As known chemical scaffolds tend to show less complexity compared to natural products, screening libraries of know chemical entities has decreased the number of novel antibiotics [147]. This is particularly concerning given the increasing reports of antibiotic resistance [148].

\subsection{Screening natural products}

Having noted the obstacles natural product research faces, this avenue should not be viewed as a dead end. The role of natural products in human health has been crucial for thousands of years [76]. Plant derived natural products have been especially crucial and the WHO estimate that a majority of the global population still rely on traditional medicines [76]. Anti-infective agents such as the $\beta$-lactams, tetracyclins, aminoglycosides, macrolides and streptogramins all play crucial roles in controlling various bacterial infections, and they all are a result of natural product research [76]. The majority of current antibiotics were discovered during the 'golden era' of antibiotic research spanning from the 1930s to the 1970s [149]. Following this timeframe the number of new classes of antibiotics dwindled, until the 2000s where five new classes of antibiotic were approved (linezolid, daptomycin, retapamulin, fidaxomicin, and bedaquiline) [149]. Of the 49 compounds under clinical development as antibiotics, 25 are of synthetic origin and 22 are of natural origin including peptide derived compounds [149]. Though these number may seem unfavourable for natural products, if we look at structural novelty, there are only a total of six novel truly synthetic compounds compared to 11 of natural product origin [149]. These examples illustrate the 
effectiveness of natural products as a source of chemical diversity, even when the number of companies actively engaged in antibiotic research has drastically declined [149].

There needs to be a clear reason for more focus in this field. There is now a slow rise in incentive for the field of antibiotic research, the US Generating Antibiotic Incentives act provides an automatic priority review and a 5-7 year market exclusivity for antibiotics that target qualifying pathogens [149]. The Innovative Medicines Initiative-New Drugs for Bad Bugs is a $\$ 280$ million dollar fund to identify new antibiotics that target Gramnegative bacteria [149]. These highlight important first steps and will require further commitments which will help towards drawing more research into this vital field.

\subsubsection{Overcoming the challenges in natural product research}

As stated earlier, approximately $74 \%$ of all approved antibacterial drugs identified between 1981-2010 originated from natural products [77]. Natural products therefore still remain a vital source for novel therapeutics [77]. Though the field remains temperamental, it is crucial that innovative methods are developed to uncover the yet undiscovered bioactive compounds that already exist, a few such methods are described below.

Cubist Pharmaceuticals have developed a method to screen multiple soil fermentation broths against strains of bacteria that are resistant to known antibiotics, to avoid isolating known compounds [139]. This approach involves screening more than $10^{7}$ strains of actinomycetes a year, using $\mathrm{Ca}^{2+}$-alginate macro-droplet beads in which bacteria are grown to produce secondary metabolites. In order to avoid re-isolating known compounds, these cultures are screened for inhibitory activity against an E. coli strain modified to be resistant to many known antibiotics [139].

Another technique, developed by Merck, employed the use of two strains of S. aureus, one wild-type and the other expressing anti-sense mRNA for FabF, an enzyme essential for bacterial fatty acid biosynthesis. The latter strain was sensitised towards fatty acid biosynthesis inhibitors. The two strains were then screened against a library of natural extracts and the hits specific to the anti-sense expressing mutant led to the eventual isolation of two unique compounds, platensimycin A (37), an inhibitor of FabF fatty 
acid acyl carrier protein synthase II [150], and platencin (38), a dual FabF/FabH inhibitor [151].

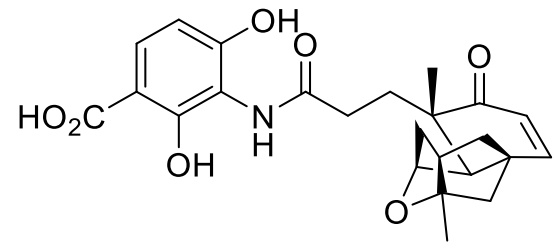

37

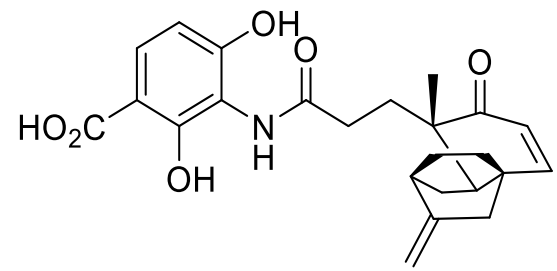

38

Bugni et al. developed methods that involved the application of hyphenated techniques to allow for the fractionation, analysis and preparation of semi-crude extracts for screens. HPLC coupled with mass spectrometry followed by the collection of compounds in HTS plates allowed for the semi-purification and identification of novel compounds with anti-tumor activity against breast cancer. This technique also separated the compounds from general cytotoxic compounds, which would be observed using traditional bioassay-guided techniques [152].

Genome mining is another method that can be employed to identify structurally diverse compounds. This technique is generally applied when dealing with secondary metabolites of microbial origin. It entails genome sequencing, followed by bioinformatics profiling to identify pathways involved in production of unique molecules. Using this method salinosporamide $\mathrm{K}$ (39) was discovery from Salinospora pacifica [153]. Additionally, cryptic biosynthetic pathways can be identified, and compounds that are not normally produced can be induced through genetic manipulation. By inducing the constant expression of a regulatory gene within the polyketide synthase (PKS) gene cluster of Streptomyces ambofaciens, stambomycins A-D, which were not produced under normal culture conditions, were able to be isolated [154]. 


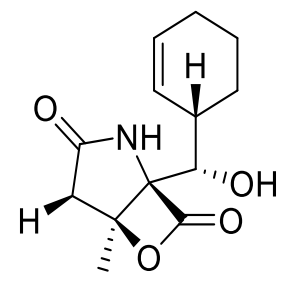

39

These examples highlight natural products and the importance they play in identifying the next generation of bioactive molecules. Two common screening approaches towards drug discovery are described below.

\subsubsection{Whole cell screening}

Whole cell screening involves measuring the growth of bacteria in the presence of potential inhibitors. Optical density (OD), green fluorescent protein (GFP) fluorescence, as well as dyes that measure metabolism such as resazurin, are often employed as measures of growth $[130,155]$. Whole cell screening has led to the identification of many current anti-tubercular drug candidates, such as PA-824 (13), a nitroimidazofuran [156] and TMC-207 (17), a diarylquinoline that has gained approval for the treatment of MDR-TB [118]. The benefit of this approach is that factors such as cell wall penetration, pro-drug activation and drug efflux pumps can be accounted for. However, the pitfall of this approach is that it is unable to account for general cytotoxicity, which is a parameter that must be evaluated at some stage. Modification of screening conditions can also be used to replicate conditions that represent in vivo environments [155]. For example, a macrophage infection model can be used to replicate the pathogenesis of TB and allow for screening and identification of inhibitors active under these conditions [157]. In this model, toxicity of the compounds towards eukaryotic cells can be accounted for, however the mode of the action of the drug still has to be elucidated.

\subsubsection{Targeted screening}

The genomic era spurred the idea of target screening; as genomes of pathogenic bacteria were sequenced it allowed for the identification of essential genes, and therefore new targets to guide the search for new antibiotics [115]. 
Enzyme/biochemical assays allow for the screening of inhibitors of a particular cellular process. Though effective, these assays are unable to account for the complexities of a whole cell [158], and the compound may not be active when tested in whole cell assays [159]. SQ-641 (40), a drug currently in pre-clinical development to treat tuberculosis, was discovered using a screen to target phospho- $N$-acetylmuramyl-pentapeptidetranslocase. It was later also found to inhibit growth of Mycobacterium smegmatis [40, $160]$.<smiles>CO[C@H]1[C@H](O)[C@H](n2ccc(=O)[nH]c2=O)O[C@H]1[C@H](O)O[C@H]1OC(C(=O)N[C@H]2CCCCNC2=O)=C[C@H](O)[C@H]1O</smiles>

40

Targeted screens also include anti-sense screens as described earlier in the isolation of platensimycin A [150]. This method, which involves the controlled expression of antisense RNA of a desired gene, makes the strain sensitive to inhibitors that target the gene of interest.

\subsubsection{Anti-tubercular drug screening at VUW}

The assay protocols developed in our laboratory were established by Drs. Miller and O'Toole. The general method involved a liquid screen of chemical libraries of known compounds such as the LOPAC, Spectrum Collection, and the NIH Diversity and Natural Products Set II libraries against M. smegmatis. By using GFP and OD, bacterial growth was measured, followed by validation of hits using dose response curves. In this protocol, culture conditions were altered in order to identify compounds that were active under various starvation conditions that emulated tuberculosis pathogenesis [155]. The 96-well plate liquid assay to screen for anti-mycobacterial activity is widely used and a well-established method [161, 162] .

As stated screening libraries of know compounds introduces very little variation in chemical structures. Diverse chemical scaffolds are a priority when dealing with diseases as imposing as TB [163]. Natural products have provided, and will continue to be, a source of structural novelty and the focus of this thesis was to utilise this powerful resource to identify such compounds. 
Applying this assay protocol, the current project involved screening NZ marine extract libraries for anti-mycobacterial activity against $M$. smegmatis. The strains of M. smegmatis employed were modified to express a GFP gene and also included a kanamycin resistance gene, which allows for the selective growth of this strain and hence reduces contamination risks by other bacteria [155]. M. smegmatis offers the advantage of a faster doubling time and poses a low health risk compared to the slower growing pathogenic mycobacteria. The extract libraries used were generated from New Zealand marine vertebrates and invertebrates. Following the identification of hit extracts, validation of anti-mycobacterial activity was performed in M. tuberculosis H37Ra. Following the selection of samples, a bioassay-guided approach was implemented focusing on the identification of potentially novel anti-mycobacterial compounds.

\subsection{Methods}

\subsubsection{Marine extract library screening}

Libraries were screened as described in section 2.6.2, with specific details for the NIWA library and VUW MNP library mentioned here. Six 96-well plates of a pre-existing marine extract library donated from NIWA were re-suspended in DMSO (500 $\mu \mathrm{L})$. From these mother plates, daughter plates were created containing $100 \mu \mathrm{L}$ aliquots of dissolved extract and were used to screen for activity. As mentioned earlier, the library from NIWA was generated with an unquantified and varying amount of extract present in each well. To screen the library, $2.5 \mu \mathrm{L}$ aliquots from each well of a daughter plate were added to $50 \mu \mathrm{L}$ of media, followed by $50 \mu \mathrm{L}$ of bacteria. The VUW MNP library was screened such that the final concentration of crude extracts was $100 \mu \mathrm{g} / \mathrm{mL}$ per well. The VUW MNP library was screened against M. smegmatis, S. aureus and E. coli. The final OD for bacteria used in screens was $0.01 \mathrm{AU}$.

\subsubsection{Validation}

Hits identified from screens were validated using dose response assays as described in sections 2.6.3 and 2.6.4. In brief, aliquots of crude extracts were diluted two-fold in appropriate media for either M. smegmatis or M. tuberculosis H37Ra, resulting in a $50 \mu \mathrm{L}$ final volume of media and extract. Bacterial cultures were diluted to ODs of 0.02 AU for M. smegmatis or 0.01 AU for M. tuberculosis $\mathrm{H} 37 \mathrm{Ra}$ with $50 \mu \mathrm{L}$ aliquots added 
to each well. For each validation assay, plates with a dilution series of a solvent control and a known anti-tubercular drug (either streptomycin or rifampicin) as positive control were included. 


\subsection{Results}

Results of screening the NIWA marine extract library and VUW MNP library are depicted below in graphical format (Figure 3.1 and 3.2). Growth inhibition is represented using GFP measurement on the $\mathrm{X}$ axis and resazurin fluorescence on the $\mathrm{Y}$ axis. A hit was defined as greater than $80 \%$ inhibition of growth as indicated by using both GFP and resazurin. This is indicated by hits that fall within the black squares in both scatter plots. Z-factors were calculated for these screens with values above 0.5 considered statistically acceptable [164]. Following screening results, tables depict the activity of the validated hits from both screens. As sigmoidal curves were not possible for these crude extracts, $\mathrm{MIC}_{90}$ values have been listed.

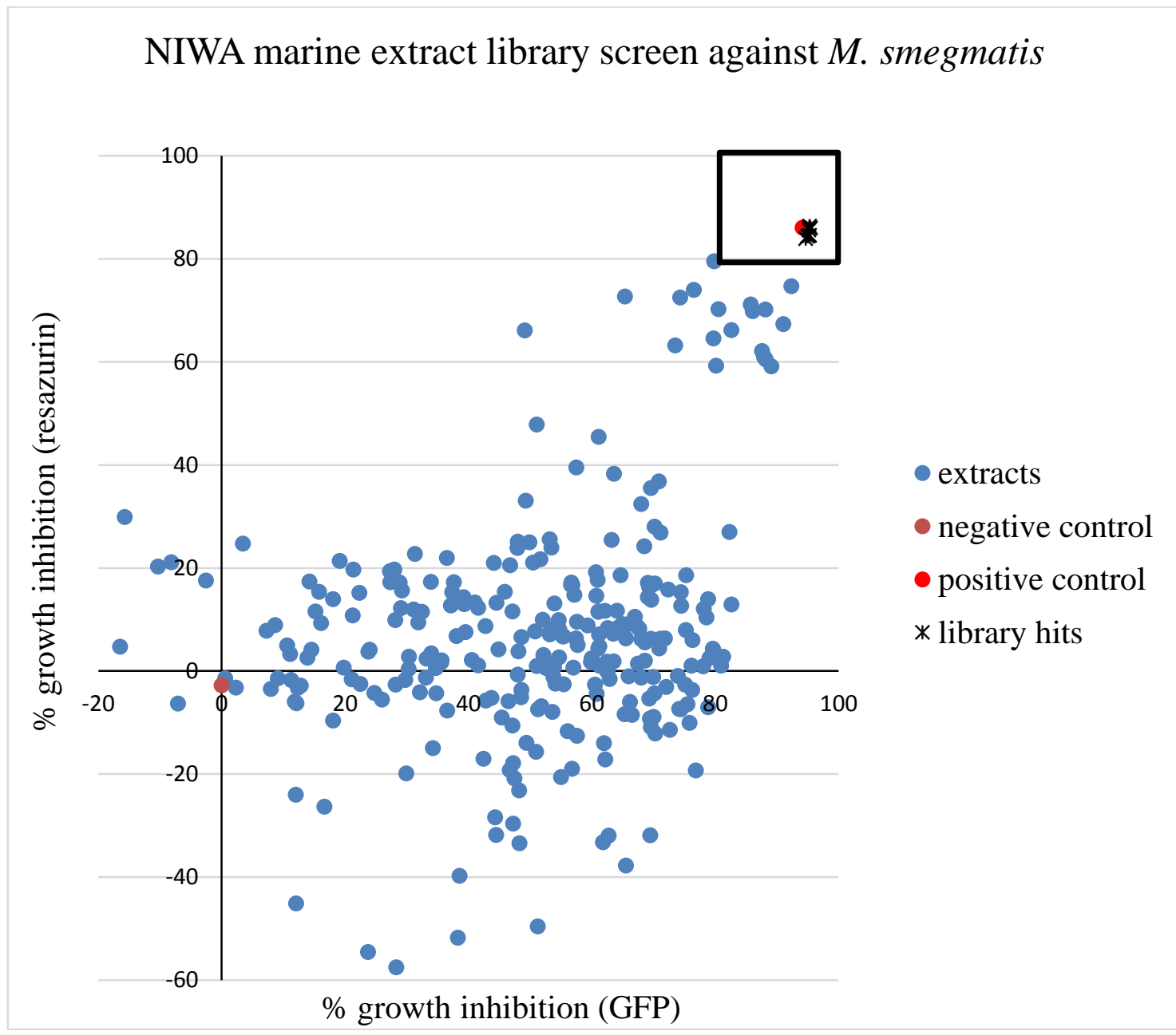

Figure 3.1 M. smegmatis screening results from the NIWA library

Scatterplot depicting the growth inhibitory effects of all extracts from the NIWA library against M. smegmatis pLL192_hsp60. Hits (indicated by asterisks) fall within the $80 \%$ cut off indicated by black square. The NIWA library screen was performed in triplicate, error bars have been excluded from the scatter plots to allow for better visualisation of data. The Z-factors calculated for the NIWA screens were $Z=0.92$ (GFP) and $Z=0.59$ (resazurin). 


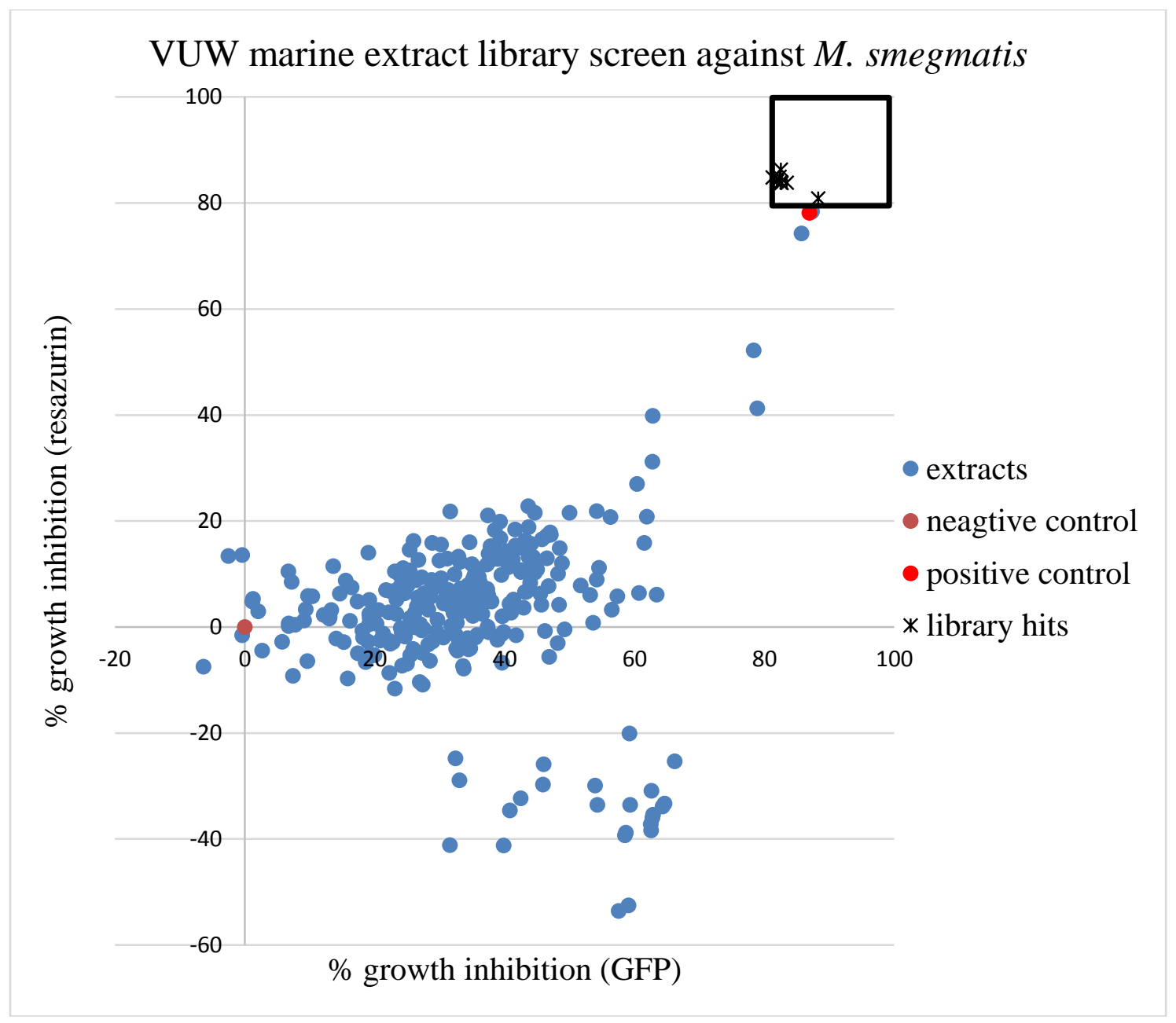

Figure 3.2 M. smegmatis screening results from the VUW MNP library

Scatterplot depicting the growth inhibitory effects of all extracts from the VUW MNP library against $M$. smegmatis pLL192_hsp60. Hits (indicated by asterisks) fall within the $80 \%$ cut off indicated by black square. Of the three VUW MNP library plates two screened in duplicate and one was in a single screen as this plate was used to optimise screening concentrations. Error bars have been excluded from the scatter plots to allow for better visualisation of data. The Z-factor for the VUW MNP screens were $Z=0.90$ (GFP) and $\mathrm{Z}=0.78$ (resazurin). 
Table 3.1 Top hits from NIWA Library

\begin{tabular}{ll}
\hline Sample & $\begin{array}{l}\text { MIC90 }(\mu \mathrm{g} / \mathrm{mL}) \\
\text { H37Ra }\end{array}$ \\
& 600 \\
\hline Lipkius holthuisi (shrimp body without & \\
carapace) & 400 \\
Starfish intestine & 250 \\
Red star fish intestine & 400 \\
Paralomis hystrix (spiky crab intestines) & 400 \\
Hoplostethus mediterraneus (silver roughy & \\
intestines) & 400 \\
Psychrolutes marcidus (blob fish intestines) & 600 \\
Psychrolutes marcidus (blob fish liver) & 1350 \\
Neolithodes brodiei (red crab intestines) & \\
\hline
\end{tabular}

Validation of the hits from the NIWA library against $M$. tuberculosis H37Ra using dose response curves, $\mathrm{MIC}_{90}$ values are the lowest concentration of extract tested that displayed $\geq 90 \%$ growth inhibition.

Table 3.2 Top hits from VUW MNP library

\begin{tabular}{ll}
\hline Sample & $\begin{array}{l}\text { MIC90 }(\mu \mathrm{g} / \mathrm{mL}) \text { vs. } \text { M. tuberculosis } \\
\text { H37Ra }\end{array}$ \\
\hline PTN 3_37C, unknown Tongan black & 200 \\
ascidian & NT \\
PTN 3_40B, unknown Tongan purple & \\
ascidian & 200 \\
PTN 2_50B, unknown Hen and Chicken & \\
Islands (Northland) Brown sponge & IA \\
PTN 2_45H, unknown Tutakaka sponge & IA \\
PTN 2_45I, unknown Tutakaka sponge & NT** \\
MNP_0999, Haliclona sp. sponge & 6.25 \\
PTN 2_56C, Latrunculia sp. sponge & 200 \\
PTN 2_93D, Xiphophora condrophylla & \\
\hline NT: not tested in M. tuberculosis H37Ra, ** MIC90 in M. smegmatis 25 $\mu \mathrm{g} / \mathrm{mL}$, IA:
\end{tabular}
MIC $_{90}$ not achievable at the highest concentration tested $(200 \mu \mathrm{g} / \mathrm{mL})$. Validation of the hits from the VUW MNP library against M. tuberculosis H37Ra using dose response curves, $\mathrm{MIC}_{90}$ values are the lowest concentration of extract tested that displayed $\geq 90 \%$ growth inhibition. 


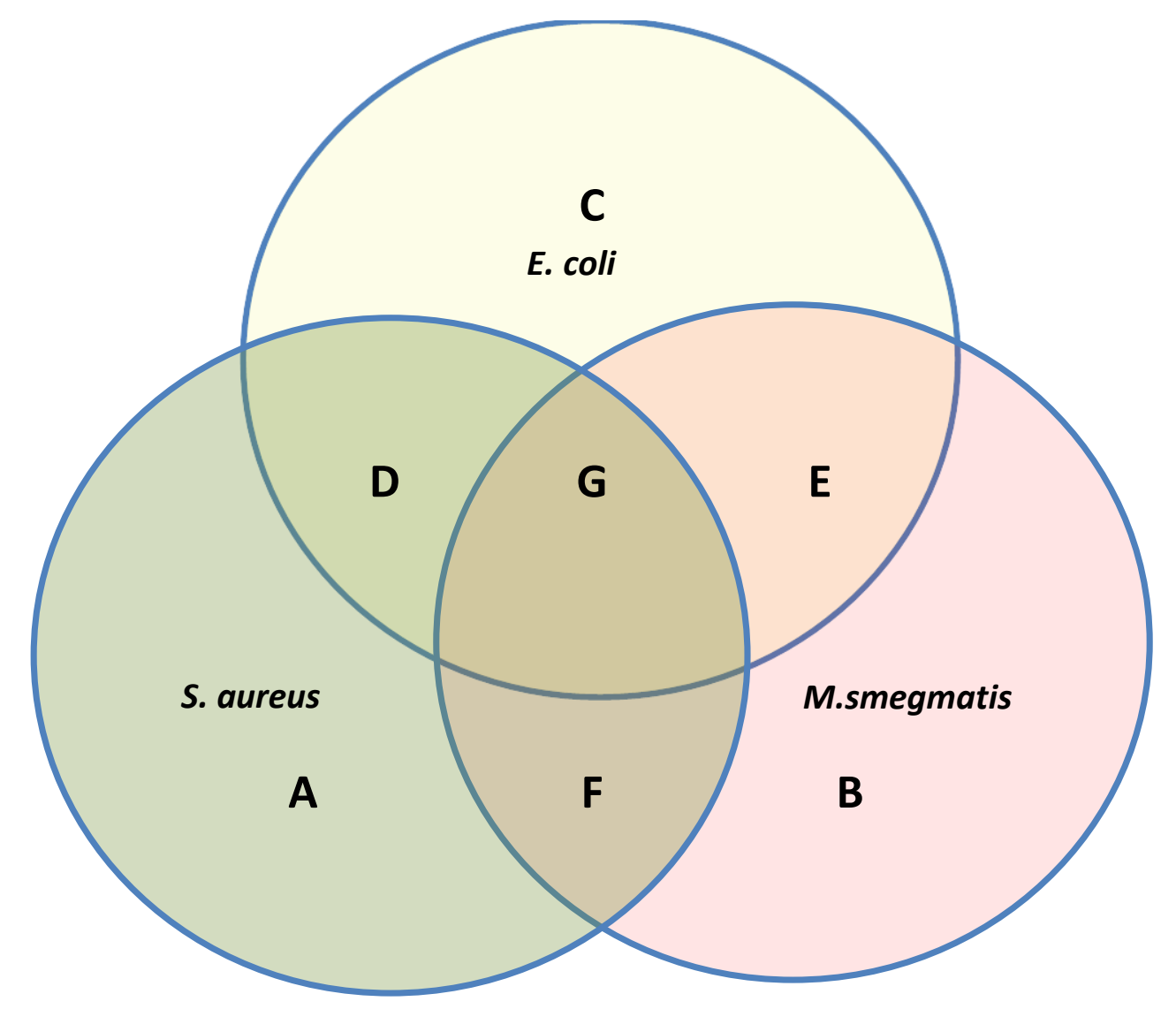

Spectrum of activity

A

PTN 2_41E, PTN 4_13H

B

PTN 3_40B, PTN 3_37C

C

$\mathrm{D}$

E

F PTN2_56C, PTN2_50B

G MNP_0999, PTN 2_93D, PTN 2_45I, PTN

$2 \_45 \mathrm{H}$

Figure 3.3 Spectrum of activity and overlap of hits from VUW MNP library screen. 
Table 3.3 Hits further investigated

\begin{tabular}{llc}
\multicolumn{1}{c}{ Sample } & \multicolumn{1}{c}{ Species } & \multicolumn{1}{c}{ MIC90 } \\
\hline Red Crab & M. smegmatis & $1 \% \mathrm{v} / \mathrm{v}$ \\
& M. tuberculosis H37Ra & $1350 \mu \mathrm{g} / \mathrm{mL}$ \\
Hen and Chicken islands & M. smegmatis & $62.5 \mu \mathrm{g} / \mathrm{mL}$ \\
brown sponge & M. tuberculosis H37Ra & $\geq 200 \mu \mathrm{g} / \mathrm{mL}$ \\
PTN 3_50B & M. smegmatis & $1 \% \mathrm{v} / \mathrm{v}$ \\
Psychrolutes marcidus & M. tuberculosis $\mathrm{H} 37 \mathrm{Ra}$ & $600 \mu \mathrm{g} / \mathrm{mL}$ \\
& M. smegmatis & $100 \mu \mathrm{g} / \mathrm{mL}$ \\
Latrunculia sp.* & M. tuberculosis $\mathrm{H} 37 \mathrm{Ra}$ & $6.25 \mu \mathrm{g} / \mathrm{mL}$ \\
PTN 2_56C & M. smegmatis & $\geq 200 \mu \mathrm{g} / \mathrm{mL}$ \\
Xiphophora chondrophylla & M. tuberculosis H37Ra & $\geq 200 \mu \mathrm{g} / \mathrm{mL}$ \\
\hline
\end{tabular}

Comparison of activity of hit extracts in two species of mycobacteria using dose response curves, $\mathrm{MIC}_{90}$ values are the lowest concentration of extract tested that displayed $\geq 90 \%$ growth inhibition. *Based on comparisons of $\mathrm{MIC}_{90}$ values, this extract shows a specificity for M. tuberculosis H37Ra. 


\subsection{Discussion}

The results from the NIWA library screening performed against M. smegmatis are provided in Figure 3.1 and for the VUW MNP library in Figure 3.2. GFP and resazurin fluorescence measurements were used as a measure of cell replication. OD was not used as measurements become unreliable due to the coloured nature of crude extracts, which contribute to OD measurements. In the NIWA library screen it is noted that many data points are negative values, where the resulting growth is higher with respect to control wells, indicating the possibility of growth promotion by the extracts in question. As the Z-factors calculated for the NIWA screens were above 0.5 this screen can be considered statistically acceptable (Figure 3.1) [164].

As stated earlier, the NIWA library was generated with an unknown mass of extract per well and as such, the library of extracts was dissolved in a constant solvent volume (500 $\mu \mathrm{L}$ DMSO). Following this the library was screened at a set $(2.5 \% \mathrm{v} / \mathrm{v})$ concentration of extract per well. This concentration was chosen as this is the highest concentration of DMSO that M. smegmatis can tolerate without solvent effects inhibiting cell growth. The unknown mass per well posed a risk of overestimating the activity of extracts that were present at higher masses in some wells than others. Once hits were identified, they were validated in dose response assays. These hits were validated by drying $100 \mu \mathrm{L}$ aliquots of the dissolved extract to identify the dry mass (masses ranged between 10$100 \mathrm{mg}$ ) before re-dissolving samples to perform dose response curves. Table 3.1 indicates the validation of the top hits from the NIWA library against M. tuberculosis H37Ra.

During optimisation of screening the VUW MNP library, no hits were observed with activity against $M$. smegmatis at 10 or $20 \mu \mathrm{g} / \mathrm{mL}$. However, at $100 \mu \mathrm{g} / \mathrm{mL}$, inhibitory activity was observed for some extracts, therefore this was used as the screening concentration. As previously stated the VUW MNP library was generated with a constant mass for each extract per well, and library screens were subsequently performed at the defined $100 \mu \mathrm{g} / \mathrm{mL}$ concentration. The Z-factors for the VUW MNP screens were above 0.5 and therefore this screen can also be considered statistically significant (Figure 3.2).

The VUW MNP library consisted of three library plates, RAK18_52A/B, 53A/B and 53C/D. RAK18_52A/B was used to optimise screening concentrations for the assay and 
due to this it was possible to only screen this plate only once rather than in duplicate as was done for RAK18_53A/B and 53C/D. As it so happened, the most active hits identified were those from RAK18_52A/B. These hits were validated in dose response assays against $M$. tuberculosis $\mathrm{H} 37 \mathrm{Ra}$ and the $\mathrm{MIC}_{90}$ values are listed (Table 3.2). It should be noted that these validations allowed for false positives to be excluded due to the lack of activity observed in this phase of testing. A possible reason for these false positives may be due to the fact that these extracts may be active against $M$. smegmatis and not M. tuberculosis H37Ra. An alternative reason for these false positive could be due to the fact they were all picked from the library plate that was screened only once; therefore there was a higher chance of false positives coming through due to random growth in wells. Fortunately the validation of hits allowed for the exclusion of false positives at an early stage, which avoided investing time into samples that may eventually fall out at later stages of the project.

Using the in-house libraries not only enabled for optimization of screening concentration but also allowed for screening the library in duplicate, as well as against S. aureus and E. coli. This allowed for the identification of samples that were specifically active against mycobacteria. Figure 3.3 identifies two such samples that showed specific activity against $M$. smegmatis. The two specific hits from the VUW MNP library screen were unfortunately not available in sufficient masses to allow for bioassay-guided fractionation.

Towards achieving the goal of Aim I, Table 3.3 identifies the top hits selected after considering sample availability as well as activity. PTN 2_50B (Hen and Chicken Islands brown sponge) was collected near the Hen and Chicken Islands, New Zealand, and was stored frozen. The crude extract had an $\mathrm{MIC}_{90}$ of $62.5 \mathrm{mg} / \mathrm{mL}$ against M. smegmatis. This sponge (250 g) was extracted twice in $\mathrm{MeOH}(450 \mathrm{~mL})$ and cyclic loaded onto HP20 and the column eluted using 20\%, 40\%, 60\%, 80\% and 100\% acetone in water. One of the most active fractions ND4_29C (280 mg) displayed an MIC 90 of 25 $\mu \mathrm{g} / \mathrm{mL}$, and was further fractionated using HP20ss (40 mL column), with mixtures of $20 \%, 40 \%, 60 \%, 70 \%, 80 \%, 90 \%$ and $100 \%$ methanol in water and finally, $100 \%$ acetone, used to elute the column. This led to fraction EJA_94D (33.6 mg) with an $\mathrm{MIC}_{90}$ of $12.5 \mu \mathrm{g} / \mathrm{mL}$, which was purified using semi-preparative reversed-phase $\mathrm{C} 18$ HPLC with $70 \% \mathrm{MeOH}$ and $0.1 \%$ formic acid under isocratic conditions to give ND4_36I (2.3mg) with an $\mathrm{MIC}_{90}$ of $50 \mu \mathrm{g} / \mathrm{mL}$. As this sample did not show any major 
improvement in activity and due to its low mass, it was not pursued any further in order to focus on other samples.

Bioassay-guided fractionation work on the liver of Psychrolutes marcidus (Blob fish, collected by NIWA) led to the identification of a mixture of fatty acids as the bioactive component. GC-MS analysis was used to analyse and identify the major components of the bioactive fraction, following which those fatty acids that were commercially available were purchased and tested for bioactivity. This work is discussed in further detail in Chapter 4.

Working with the Latrunculia sp. sponge, collected near Rimariki Island led to the isolation of a novel taurinated terpene. Based on the bioactivity of the initial fractionation, this taurinated terpene was believed to be the component responsible for the observed anti-mycobacterial activity. However, final purification of the bioactive fraction using size exclusion chromatography resulted in the separation of the taurinated terpene from the anti-mycobacterial component. This component was not isolated in a mass sufficient enough to allow for structure elucidation. Details of this work are further elaborated in Chapter 5.

Bioassay-guided fractionation of a brown alga Xiphophora chondrophylla, collected near the Hen and Chicken Islands, led to the isolation of a new 1-deoxysphingoid. This compound has an $\mathrm{MIC}_{90}$ of $65.1 \mu \mathrm{M}$ against S. aureus, E. coli, M. smegmatis and M. tuberculosis H37Ra. However, the bioactivity of the 1-deoxysphingoid was more potent versus mammalian cell lines. Therefore, the Saccharomyces cerevisiae gene knock-out library was used to identify pathways that were involved in the mechanism of action of this compound. Further details of this work are described in Chapter 6. 


\section{Chapter 4 Bioactive fatty acids from Psychrolutes marcidus}




\subsection{Background}

Following a screen against the NIWA library, the liver extract of the blob fish Psychrolutes marcidus was identified as being active. After subsequent validations in dose response assays, this sample was chosen to be pursued further. Upon collection of more specimens by NIWA, the livers were dissected and extracted to allow for isolation of any bioactive compounds responsible for inhibitory activity. This led to the identification of a mixture of fatty acids as the source of bioactivity.

\subsubsection{Fatty acids}

Fatty acids are ubiquitous in nature and play important roles within a cell such as energy reserves in the form of triglycerides, or in the cell membrane as phospholipids. Through the action of enzymes such as lipases, lipids can be cleaved from their polar head groups (such as sugars or phosphates), releasing free fatty acids [165]. It has been reported that free fatty acids (FFAs) possess a range of activities including antibacterial, anti-fungal, and cytotoxic properties [165]. Within biological systems, chain lengths ranging from 10 to 28 carbons are common for fatty acids [165]. When there is one or more double bonds within the carbon chain, they are classed as unsaturated fatty acids. In humans, FFAs act as an innate defence against pathogens on skin and mucosal surfaces and it is believed that there are approximately $10-15 \mu \mathrm{g}$ of FFAs per square centimetre of skin $[165,166]$. Their antibacterial action on the skin may be the result of direct killing of bacteria, or by decreasing the $\mathrm{pH}$ on the skin surface, making conditions unfavourable for bacterial growth [167, 168].

Antimicrobial FFAs from a number of sources such as algae, plants and animals have been isolated using bioassay-guided fractionation [169-172]. FFAs are also believed to be the active component of some traditional remedies [171, 172].

A general trend in literature indicates that antibacterial activity of FFAs depends on the carbon chain length, degree of unsaturation as well as the positioning and geometry of the double bond, with a cis geometry favouring more potent activity [173-175]. Most active FFAs have chain lengths of either 14 or 16 carbons [173]. Correlations have been observed identifying unsaturated FFAs as more active compounds compared to saturated FFAs of similar chain length [173]. In addition, a free carboxyl group is believed to be essential for activity $[173,176]$. Gram-positive bacteria are more 
susceptible to growth inhibition in the presence of FFAs compared to Gram-negative bacteria, however it should be noted that most research has only focused on Grampositive bacteria $[175,177,178]$.

\subsubsection{Nomenclature of fatty acids}

Fatty acids are named using set abbreviated nomenclature; the number of carbons in the chain is first stated followed by the number of double bonds. For example, palmitic acid, a saturated fatty acid with a chain of 16 carbon atoms, would be C16:0, whereas palmitoleic, which has the same chain length but one double bond is C16:1. In addition, the bond geometry is also stated. Therefore, for palmitoleic acid, C16:1, cis-9 would be used, which identifies a 16 carbon long chain, with one $c i s$-double bond at the $9^{\text {th }}$ carbon.

\subsubsection{Mechanism of action}

While there has been extensive literature published on the effects of FFAs, only the mechanisms that would lead to the most detrimental effects on a cell will be discussed. While the exact mechanisms of action are not fully elucidated, it has been proposed that FFA activity is potentiated in multiple ways. The presence of a hydrophilic polar head and a hydrophobic tail makes these molecules amphiphilic. Therefore, they can cause cell lysis by affecting membrane permeability as with detergents [179, 180]. Oleic acid has been shown to increase membrane fluidity in S. aureus [181].

In altering membrane permeability, FFAs can affect processes integral to cell viability such as the electron transport chain and enzyme activity. Direct interaction with electron carriers, which disrupts the electron transport chain, or alteration of membrane fluidity, can also perturb cell homeostasis in bacteria $[182,183]$.

FFAs are believed to uncouple oxidative phosphorylation, which is based on research focusing on mitochondria and has been used to draw comparisons to antibacterial activity [165, 184, 185]. This is achieved by disrupting the proton motive force generated by the electron transport chain, which is essential for ATP synthase to convert ADP to ATP [186]. FFAs have been shown to directly interact with the permeability transition pore within the mitochondrial membrane, therefore uncoupling oxidative phosphorylation [187]. Further disruption of this process is believed to occur via the dissociation of the proton from the acid group of a FFA within the cytosol, thus 
decreasing the $\mathrm{pH}$ within the cytosol. This is facilitated via anion transporters within the mitochondrial membrane [188].

An alternative mechanism proposed for the activity of FFAs includes the direct interaction and inhibition of enzymes, with reports of unsaturated FFAs being more potent than saturated FFAs [189, 190]. Within the literature, FFAs have also been linked to inhibition of fatty acid biosynthesis, which is essential for maintaining membrane integrity within bacteria [191, 192].

The varying susceptibility of bacteria to FFAs is believed to be due to the differences in permeability of bacterial cell walls, which alters FFA access to the inner membrane [165]. Some bacteria are able to resist inhibition by FFAs, by modifying their cell wall to make it more charged and therefore more hydrophilic and unfavourable for FFA binding [193]. S. aureus has been shown to upregulate genes involved in cell wall synthesis in the presence of FFAs [183]. The presence of carotenoids allows for membrane stability, negating the effects of FFA. Strains of $S$. aureus with high levels of carotenoids have been reported to display more resistance to FFAs compared to strains with lower levels [181]. 


\subsection{Methods}

\subsubsection{Extraction of material}

Blob fish were dissected and livers extracted. Livers $(200 \mathrm{~g})$ were cut into $1 \mathrm{~cm}^{3}$ sized pieces and soaked in methanol $(\sim 200 \mathrm{~mL})$. Following overnight extraction, the methanol was decanted into a conical flask and the liver material was re-extracted with the same volume of methanol. The two extracts were treated separately and filtered using a Büchner funnel and Celite. The second extract was passed through an $80 \mathrm{~mL}$ HP20 column followed by the first. The eluents were combined and diluted two-fold with $\mathrm{H}_{2} \mathrm{O}$ before passing through the same column again. This was repeated until the original $\mathrm{MeOH}$ extract was diluted to $12.5 \%$ and passed through the column. Following cyclic loading, the column was eluted with $240 \mathrm{~mL}$ of $20 \%$ and $40 \%$ acetone in water followed by $60 \%$ and $100 \%$ acetonitrile in water, and finally $100 \%$ acetone was used to strip the column.

From the $100 \%$ acetonitrile fraction (12.6 grams of oil, $\left.\mathrm{MIC}_{90} 2 \% \mathrm{v} / \mathrm{v}\right), 100 \mathrm{mg}$ was further fractionated using a $10 \mathrm{~mL}$ DIOL column, eluting with $30 \mathrm{~mL}$ of $100 \%$ hexanes, $75 \%, 50 \%, 25 \%$ mixtures of hexanes in EtOAc, 100\% EtOAc, 75\%, 50\%, 25\% mixtures of EtOAc and $\mathrm{MeOH}, 100 \% \mathrm{MeOH}$ and column strip of $50 \% \mathrm{MeOH}$ and $\mathrm{H}_{2} \mathrm{O}$. Only the $100 \%$ hexanes and the column strip were collected in bulk, whereas the other eluents were collected in test tubes. Test tubes were dried and combined based on charring $\left(5 \% \mathrm{H}_{2} \mathrm{SO}_{4}\right.$ in $\left.\mathrm{MeOH}\right)$ of TLC (running condition 4:1:2 butanol: acetic acid:water). From the resulting fractions, ND4_43B (116.2 mg, MIC90 $250 \mathrm{mg} / \mathrm{mL}$ ) was separated by LH20 size exclusion chromatography using 50\% MeOH/ 50\% DCM. This gave ND5_96L as the active fraction $\left(2.5 \mathrm{mg} \mathrm{MIC}_{90} 250 \mu \mathrm{g} / \mathrm{mL}\right)$. 


\subsubsection{Cyclic loading and column purification}

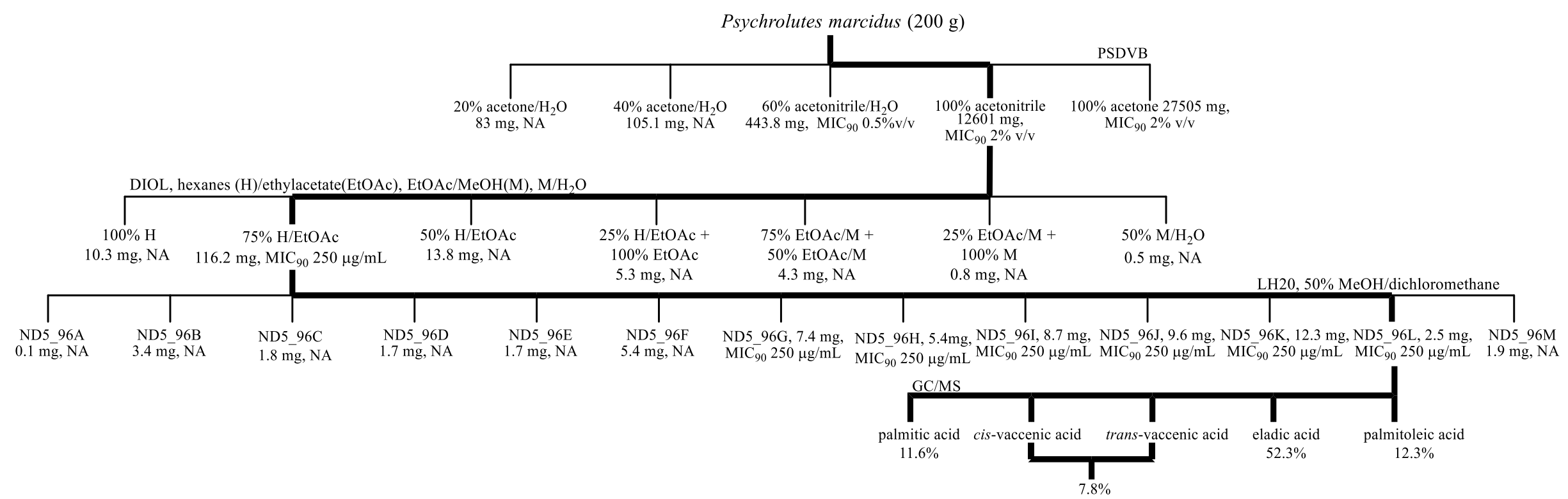

Scheme 4.1 Isolation scheme of fatty acids from Psychrolutes marcidus 


\subsubsection{GC-MS analysis of fatty acids}

A common method to analyse fatty acids is to derivatise them and generate fatty acid methyl esters (FAMEs), which are then analysed via GC-MS [194]. Fatty acid methyl esters were generated by adding $1 \mathrm{~mL}$ of a $10 \%$ solution of boron trifluoride in methanol to $100 \mu \mathrm{g}$ of ND5_96L. This mixture was heated for 10 minutes at $90{ }^{\circ} \mathrm{C}$, followed by liquid-liquid partitioning with water and chloroform three times. The chloroform layer was dried for analysis by GC-MS (Integrated Bioactive Technologies group, Callaghan Innovation). Chloroform (45 mL) was added to the sample and analysis was conducted on a Shimadzu QP2010 GC-MS/FID, using a Thermo TGWaxA column (30 m, $0.25 \mathrm{~mm}$ i.d., $0.25 \mu \mathrm{M}$ film), with isothermal conditions at $210{ }^{\circ} \mathrm{C}$, and the injector temperature set at $280{ }^{\circ} \mathrm{C}$ using a 50:1 split. Helium was used as the carrier gas. Mass spectrometry fragmentation patterns (electron impact ionisation at $70 \mathrm{eV}$ in positive ion mode) as well as equivalent chain length values were used to identify peaks.

\subsubsection{Fatty acids}

The following fatty acids identified using GC/MS, were sourced from Sigma: eladic acid, petroselenic acid, oleic acid, cis-vaccenic acid, trans-vaccenic acid, linoleic acid, palmitic acid and palmitoleic acid; all fatty acids were of $\geq 99.0 \%$ purity. These fatty acids were either tested individually or in mixtures of two, three or four. Fatty acid mixtures were generated using equal proportions of each fatty acid within each respective mixture. 


\subsection{Results}

Using the methodology described in section 2.6, the results of the dose-response assays for the active fraction as well as fatty acids and their mixtures are listed below. Graphs were generated using GraphPad Prism v.5.0. Resazurin dye was used to measure bacterial metabolism after each assay was run, and fluorescence measurements (RFU) were used as a measure of growth. OD was used as measure of growth for the HL-60 and HEK MTT assay as well as the S. cerevisiae assay. As stated earlier, where possible, $\mathrm{IC}_{50}$ values have been listed, however as sigmoidal curves were not achievable for all data, $\mathrm{MIC}_{90}$ values have been listed as well. 


\subsubsection{GC/MS results}

Table 4.1 GC/MS profile of FAMEs in ND5_96L

\begin{tabular}{lll}
\hline & \multicolumn{2}{l}{ ND5_96L FAME } \\
Chain length & ECL (16,18) & \% FA \\
\hline & 13.84 & 0.1 \\
$14: 0$ & 13.97 & 0.34 \\
$15: 0$ & 14.99 & 0.27 \\
& 15.5 & 0.17 \\
$16: 0$ & 16.00 & 11.58 \\
$16: 1 \mathrm{n}-9$ & 16.27 & 2.05 \\
$16: 1 \mathrm{n}-7$ & 16.33 & 12.25 \\
$16: 1 \mathrm{n}-5$ & 16.45 & 0.19 \\
I17:0 & 16.51 & 0.34 \\
& 16.60 & 0.22 \\
& 16.69 & 0.18 \\
& 16.93 & 0.19 \\
$17: 0$ & 17.00 & 0.14 \\
$17: 1$ & 17.29 & 1.64 \\
& 17.41 & 0.29 \\
$18: 0$ & 17.49 & 0.37 \\
$18: 1 \mathrm{n}-9$ & 18.00 & 1.83 \\
$18: 1 \mathrm{n}-7$ & 18.25 & 52.25 \\
& 18.32 & 7.77 \\
& 18.44 & 0.44 \\
$20: 1$ & 19.26 & 0.19 \\
$20: 1$ & 19.30 & 0.47 \\
Phthalate & 20.16 & 0.28 \\
& 20.20 & 2.05 \\
& 20.72 & 3.91 \\
& 22.57 & 0.49 \\
\hline
\end{tabular}

Fatty acid composition of ND5_96L determined by FAME analysis. Estimated chain length (ECL) values indicate the retention time of each fatty acid on the GC column in relation to their saturated fatty acid counterparts. 


\subsubsection{Bioactivity}

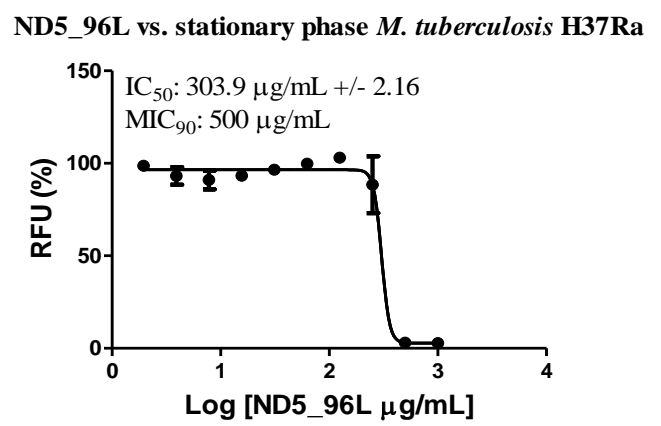

Figure 4.1 ND5_96L vs. stationary phase M. tuberculosis H37Ra

Dose response curve of ND5_96L using stationary phase assay conditions. RFU units were used as a measure of growth on the Y-axis. +/- value indicates standard error. $\mathrm{MIC}_{90}$ value is two-fold greater in stationary phase cells compared to actively growing cells. $(\mathrm{n}=1$, triplicate)

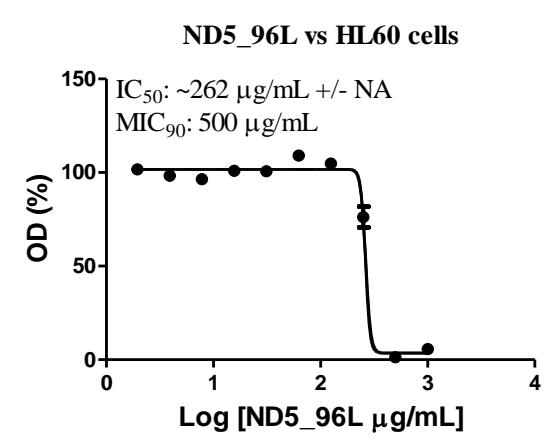

A

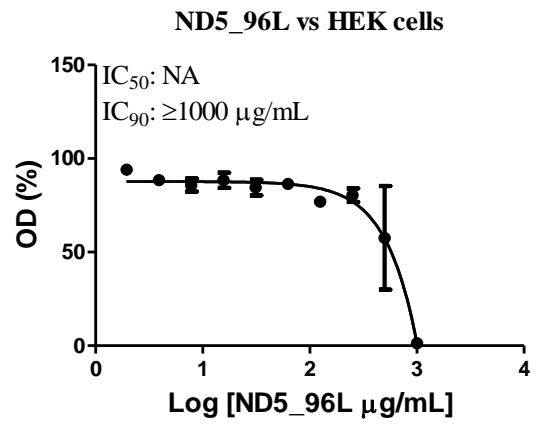

B

Figure 4.2 ND5_96L vs. mammalian cell lines

MTT assay of ND6_96L against HL-60 (A) and HEK cell lines (B). The HEK cell line has been used as an indication of general cytotoxicity. OD was used as a measure of growth on the Y-axis. NA not achievable, +/- value indicates standard error. $(\mathrm{n}=1$, duplicate $)$ 
Table 4.2 Broad- spectrum activity of active mixture of fatty acids

\section{Microorganism}

\section{Activity}

S. aureus

E. coli

P. aeruginosa

M. smegmatis

M. tuberculosis $\mathrm{H} 37 \mathrm{Ra}$

$+$

S. cerevisiae

HL60 cells

HEK cells

(+) indicates activity, (-) indicates no observed activity. Activity is defined by $\geq 90 \%$ growth inhibition observed at the highest concentrations tested $(500-1000 \mu \mathrm{g} / \mathrm{mL})$.

Table 4.3 Bioactivity profile of individual fatty acids

\begin{tabular}{llc}
\hline Name & Chain length & MIC $_{90}(\mu \mathrm{g} / \mathrm{mL})$ \\
\hline Palmitic acid & C16:0 & 250 \\
Palmitoleic acid & C16:1 & 500 \\
Petroselenic acid & C18:1, cis-6 & $\geq 1000$ \\
Oleic acid & C18:1, cis-9 & $\geq 1000$ \\
Eladic acid & C18:1, trans-9 & 500 \\
cis-vaccenic acid & C18:1, cis-11 & 500 \\
trans- vaccenic acid & C18:1, trans-11 & 500 \\
Linoleic acid & C18:3, cis-19,12,15 & NA \\
\hline
\end{tabular}

$\mathrm{MIC}_{90}$ values of fatty acids against $M$. tuberculosis $\mathrm{H} 37 \mathrm{Ra}$.

Table 4.4 Comparison of MIC90 values of artificial mixtures against M. tuberculosis H37Ra

\begin{tabular}{lc}
\hline Name & $\mathrm{MIC}_{90}(\mu \mathrm{g} / \mathrm{mL})$ \\
\hline cis mix & 250 \\
trans mix & 125 \\
ND5_96L & 250
\end{tabular}

Comparison of activities of the mixtures of the four fatty acids with either cis or transvaccenic acid to ND5_96L. 
Table 4.5 Bioactivity of fatty acid mixtures vs. M. tuberculosis H37Ra

\begin{tabular}{|c|c|c|}
\hline Name & Chain lengths & $\begin{array}{c}\mathrm{IC}_{50} \\
(\mu \mathrm{g} / \mathrm{mL}) \pm \\
\text { std. err. }\end{array}$ \\
\hline Palmitic acid & C16:0 & $66+/-1.1$ \\
\hline Palmitoleic acid & C16:1, cis-9 & $274+/-1.1$ \\
\hline Eladic Acid & C18:1, trans-9 & $301+/-1.1$ \\
\hline trans-vaccenic acid & C18:1, trans-11 & $306+/-1.1$ \\
\hline cis-vaccenic acid & C18:1, cis-11 & $166+/-1.1$ \\
\hline Palmitic/palmitoleic & C16:0/ C16:1, cis-9 & $51+/-1.1$ \\
\hline Palmitic/eladic & C16:0/C18:1, trans-9 & $50+/-1.1$ \\
\hline Palmitic/trans-vaccenic & $\mathrm{C} 16: 0 / \mathrm{C} 18: 1$, trans-11 & $48+/-1.1$ \\
\hline Palmitic/cis-vaccenic & C16:0/ C18:1, cis-11 & $102+/-1.1$ \\
\hline Palmitoleic/eladic & C16:1, cis-9/C18:1, trans-9 & $73+/-1.1$ \\
\hline Palmitoleic/trans-vaccenic & C16:1, cis-9/C18:1, trans- 11 & $64+/->1$ \\
\hline Palmitoleic/cis-vaccenic & C16:1, cis-9/ C18:1, cis-11 & $104+/-1.3$ \\
\hline Eladic/trans-vaccenic & C18:1, trans-9/ C18:1, trans-11 & $71+/-1.2$ \\
\hline Eladic/cis-vaccenic & C18:1, trans-9/C18:1, cis- 11 & $113+/-1.0$ \\
\hline Palmitic/palmitoleic/eladic & C16:0/C16:1, cis-9/C18:1, trans-9 & $60+/-1.1$ \\
\hline $\begin{array}{l}\text { Palmitic/palmitoleic/trans- } \\
\text { vaccenic }\end{array}$ & C16:0/ C16:1, cis-9/C18:1, trans-11 & $68+/-1.1$ \\
\hline Palmitic/palmitoleic/cis-vaccenic & $\begin{array}{l}\text { C16:0/C18:1, trans-9/C18:1, cis-11 } \\
\text { C16:0/C18:1, trans-9/C18:1, trans- }\end{array}$ & $125+/->1$ \\
\hline Palmitic/eladic/trans-vaccenic & 11 & $69+/-1.1$ \\
\hline Palmitic/eladic/cis-vaccenic & C16:0/C18:1, trans-9/ C18:1, cis-11/ & $79+/-1.1$ \\
\hline $\begin{array}{l}\text { Palmitoleic/eladic/trans- } \\
\text { vaccenic }\end{array}$ & $\begin{array}{l}\text { C16:1, cis-9/C18:1, trans-9/C18:1, } \\
\text { trans-11 } \\
\text { C16:1, cis-9/C18:1, trans-9/C18:1, }\end{array}$ & $72+/-1.1$ \\
\hline Palmitoleic/eladic/cis-vaccenic & cis-11 & $81+/-1.3$ \\
\hline trans-vaccenic mix of the & C16:0/C16:1, cis-9/C18:1, trans-9/ & \\
\hline original & $\begin{array}{l}\text { C18:1, trans-11 } \\
\text { C16:0/C16:1, cis-9/C18:1, trans-9/ }\end{array}$ & $76+/-1.1$ \\
\hline cis-vaccenic mix of the original & C18:1, cis-11 & $102+/-1.1$ \\
\hline
\end{tabular}




\subsection{Discussion}

\subsubsection{Fractionation and isolation}

An initial crude fractionation was performed using HP20 (PSDVB) column chromatography (Scheme 4.1). Based on the anti-mycobacterial activity of the fractions generated, the $100 \%$ acetonitrile fraction was chosen for the next stage of purification, using DIOL normal phase chromatography. Based on its profile, the $75 \%$ hexanes / EtOAc fraction was selected to undergo the final stage of size exclusion chromatography using LH20 gel. Based on the bioactivity as well as the ${ }^{1} \mathrm{H}$ NMR spectra of the preceding fractions, ND5_96L was identified as the most potent fraction, however it was apparent that it was still a mixture of fatty acids.

\subsubsection{Structural elucidation}

The initial method used to identify the structure of the bioactive compound was NMR spectroscopy. Using NMR, it was concluded that the most pure and active fraction, ND5_96L, was a mixture of C-18 fatty acids. Therefore, a range of C-18 fatty acid standards were purchased, spectra were acquired and these were compared to ND6_96L. Based on ${ }^{1} \mathrm{H}$ and ${ }^{13} \mathrm{C} \mathrm{NMR}$ data, it was initially concluded that the sample was a mixture of oleic and cis-vaccenic acids (Figure 4.3 to 4.7 ). 


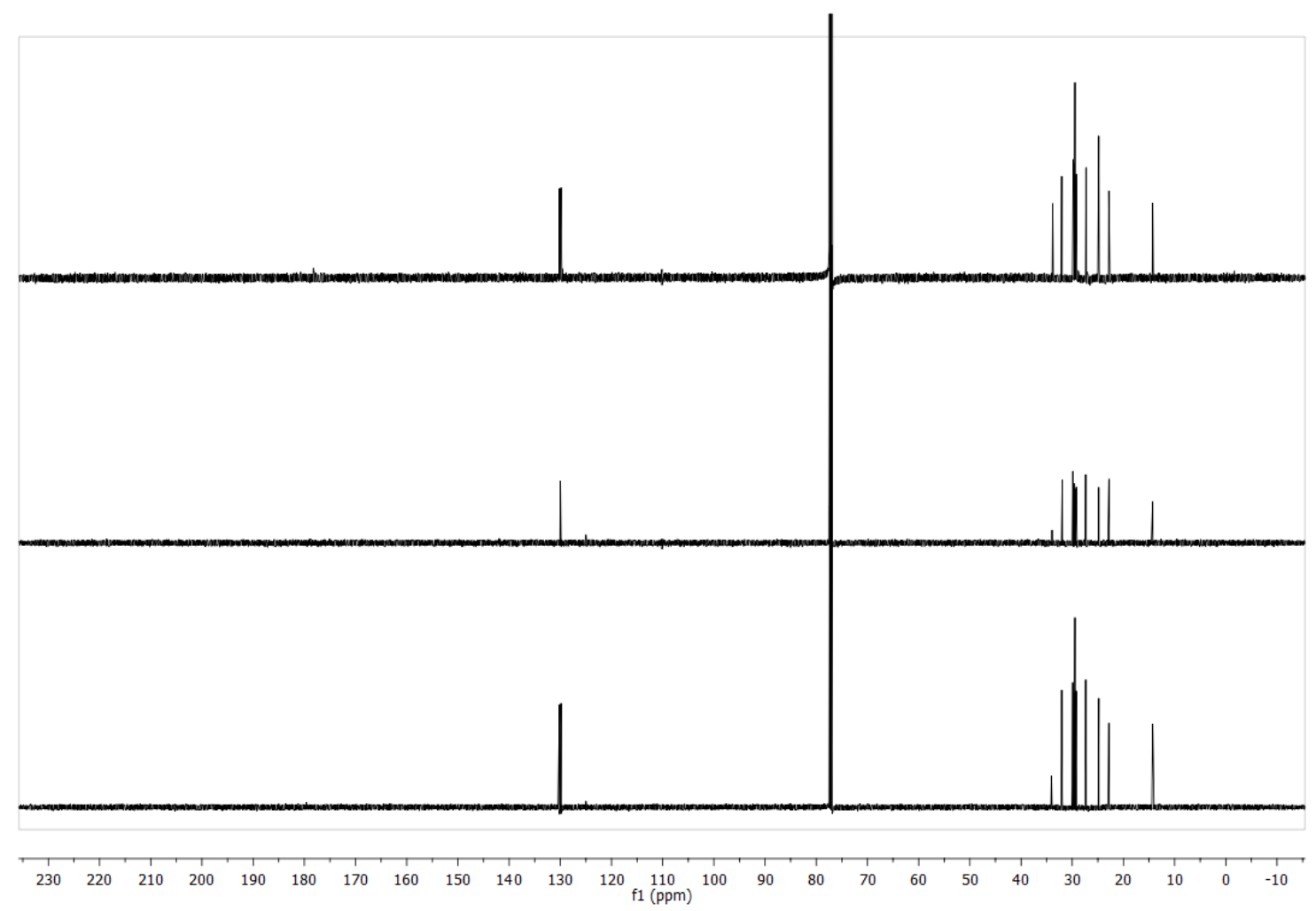

Figure 4.3 Comparison of ${ }^{13} \mathrm{C}$ NMR $\left(\mathrm{CDCl}_{3}, 150 \mathrm{MHz}\right)$ spectra of ND5_96L (top), cis-vaccenic (middle) and oleic acids (bottom) at full spectral width.

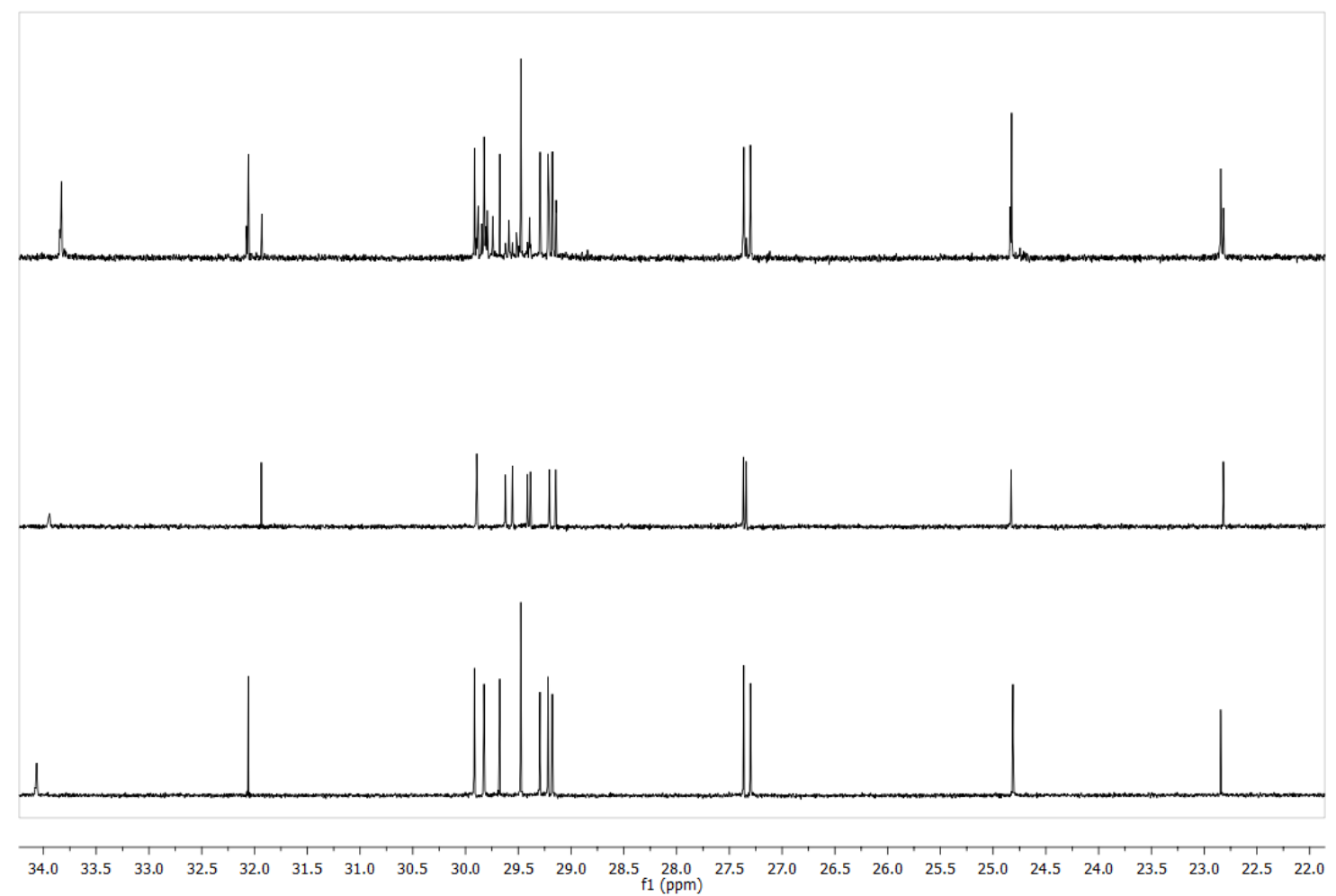

Figure 4.4 Comparison of ${ }^{13} \mathrm{C}$ NMR ( $\left.\mathrm{CDCl}_{3}, 150 \mathrm{MHz}\right)$ spectra of ND5_96L (top), cis-vaccenic (middle) and oleic acids (bottom) at a specific range of interest. 


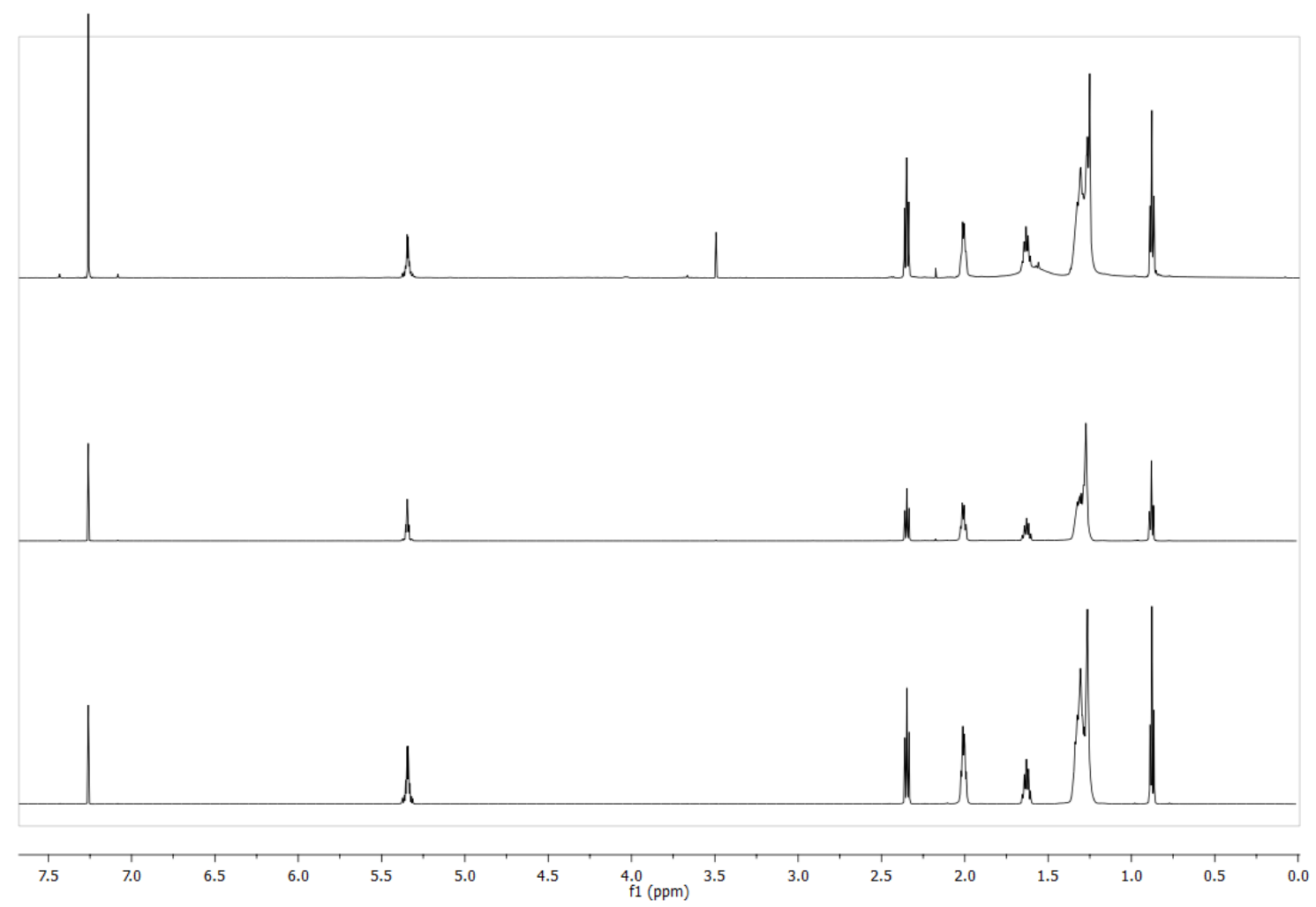

Figure 4.5 Comparison of ${ }^{1} \mathrm{H}$ NMR $\left(\mathrm{CDCl}_{3}, 600 \mathrm{MHz}\right)$ spectra of ND5_96L (top), cis-vaccenic (middle) and oleic acids (bottom) at full spectral width.

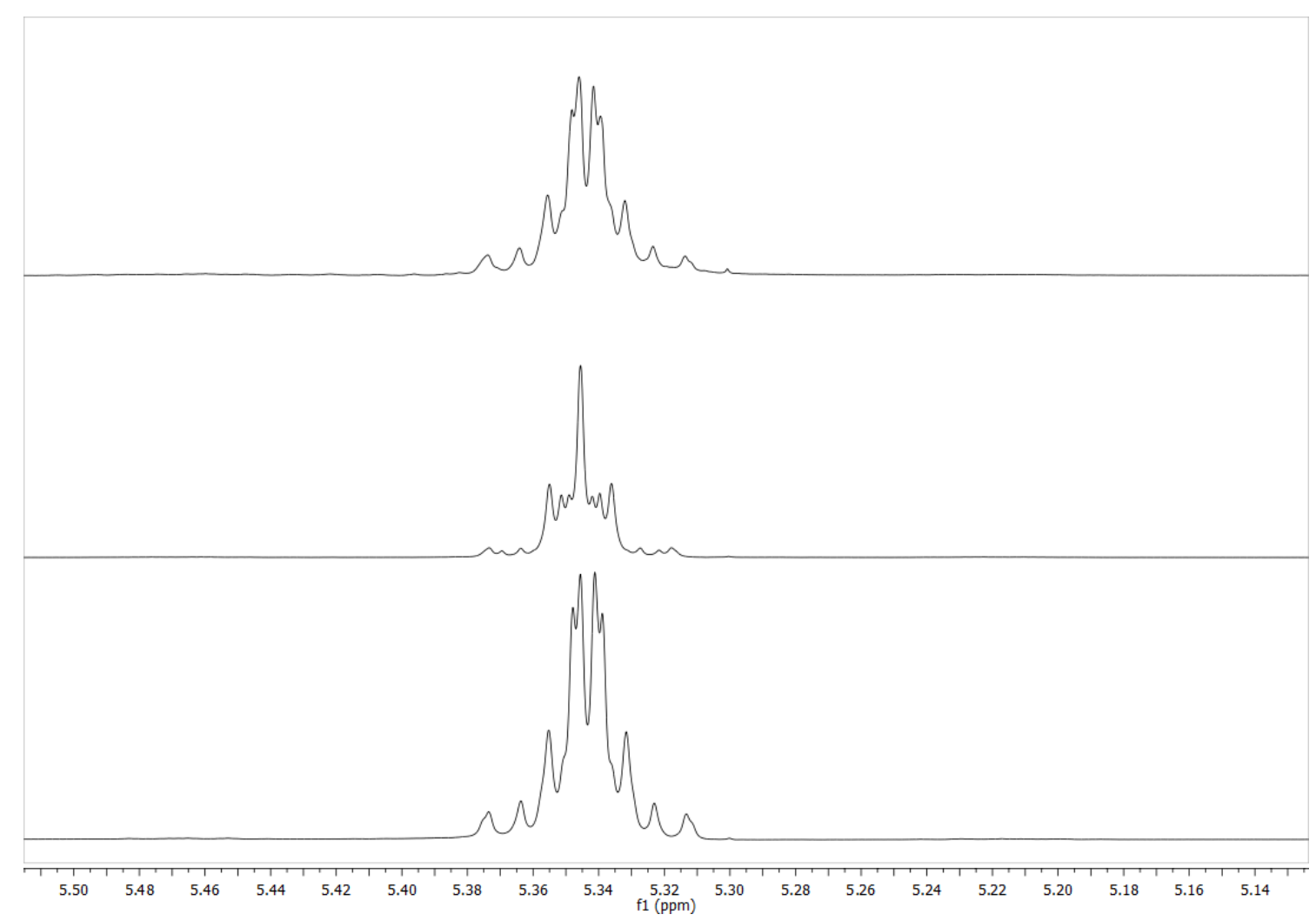

Figure 4.6 Comparison of ${ }^{1} \mathrm{H}$ NMR ( $\left.\mathrm{CDCl}_{3}, 600 \mathrm{MHz}\right)$ spectra of ND5_96L (top), cis-vaccenic (middle) and oleic acids (bottom) at a specific range of interest. 


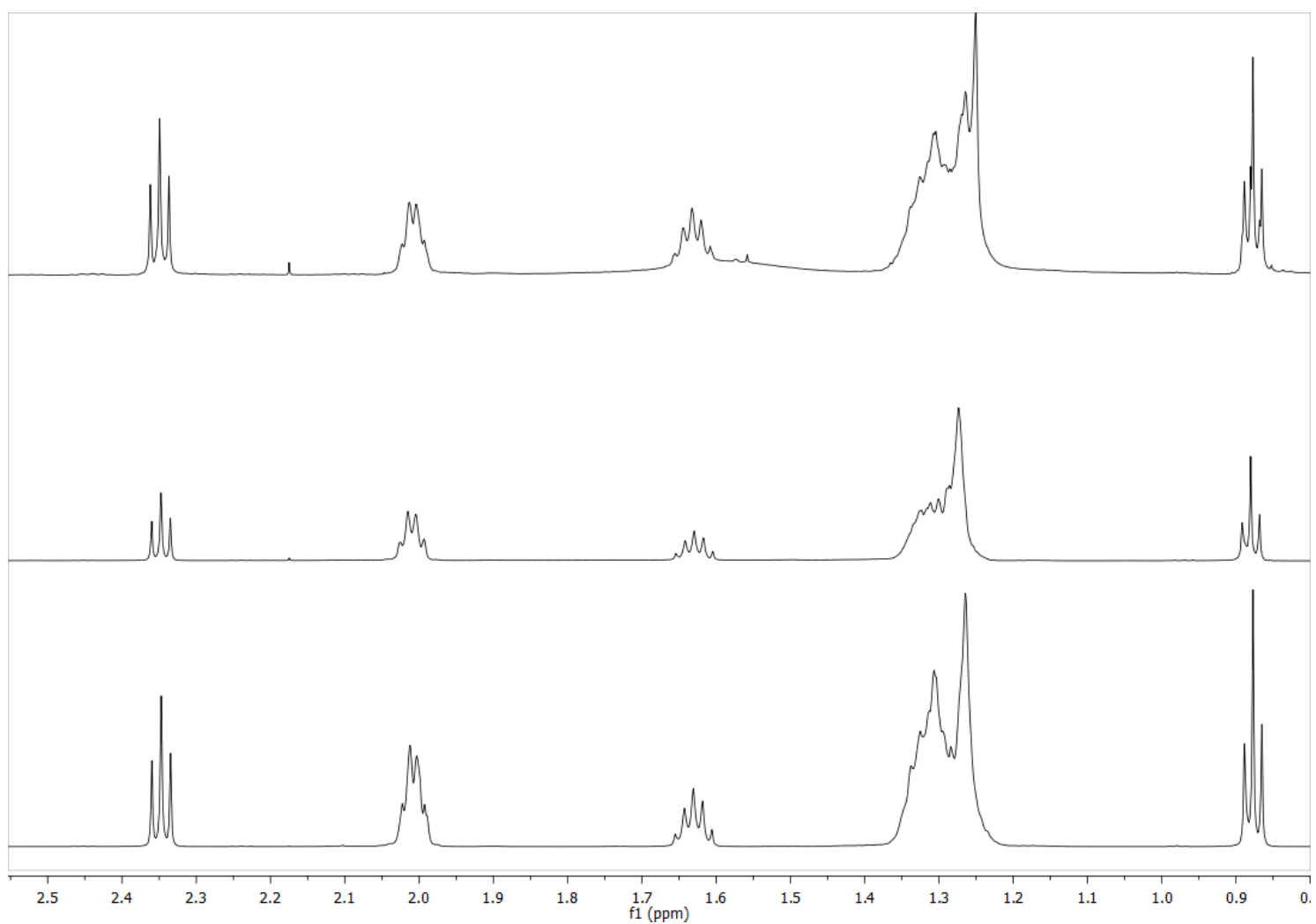

Figure 4.7 Comparison of ${ }^{1} \mathrm{H}$ NMR $\left(\mathrm{CDCl}_{3}, 600 \mathrm{MHz}\right)$ spectra of ND5_96L (top), cis-vaccenic (middle) and oleic acids (bottom) at a specific range of interest.

Testing the C-18 fatty acid standards identified MIC90 values of $1000 \mu \mathrm{g} / \mathrm{mL}$ for oleic acid and $500 \mu \mathrm{g} / \mathrm{mL}$ for cis-vaccenic acid (Table 4.3). The lack of correlation of the activities of the standards to ND5_96L ( $\mathrm{MIC}_{90} 250 \mu \mathrm{g} / \mathrm{mL}$ ) could be due to two factors; either the fatty acids in the mixture had not been correctly identified, or the fatty acids need to be present together in a certain ratio to be active. To address the issue of misidentification of the appropriate fatty acids, the mixture was methylated with a $10 \%$ solution of $\mathrm{BF}_{3}$ in methanol to generate the corresponding fatty acid methyl esters (FAMEs) for analysis by GC-MS. The FAME analysis identified the mixture as eladic (C18:1 trans-9) $(52.3 \%)$, vaccenic (C18:1 cis or trans-11) $(7.8 \%)$, palmitic (C16:0) (11.6\%) and palmitoleic (C16:1 cis-9) (12.3\%) acids (Table 4.1). This means the initial conclusion of the active mixture as a binary mixture of fatty acids was incorrect.

Given vaccenic acid can exist in two different geometries, two sets of mixtures were generated for bioassay testing, one using cis-vaccenic acid while the other used transvaccenic acid combined with the other three acids. All possible combinations of two or 
three fatty acids, in addition to the individual fatty acids, were tested for their bioactivity against $M$. tuberculosis H37Ra (Table 4.5).

\subsubsection{Bioactivity}

In theory, the mixture of four fatty acids should be approximately as active as ND5_96L and based on the bioassay data this is the case with an $\mathrm{MIC}_{90}$ of $125 \mu \mathrm{g} / \mathrm{mL}$ for the trans-vaccenic acid mixture and an $\mathrm{MIC}_{90}$ of $250 \mu \mathrm{g} / \mathrm{mL}$ for the $c i s$-vaccenic acid mixture (Table 4.4). These values are consistent with the observed activity of the 'natural mixture' ND6_96L $(250 \mu \mathrm{g} / \mathrm{mL})$, and based on this it could be concluded that the natural mixture contains cis-vaccenic acid.

Testing the fatty acids individually identified palmitic acid as the most biologically active with an $\mathrm{IC}_{50}$ of $66 \mu \mathrm{g} / \mathrm{mL}$ (Table 4.5). When comparing the $\mathrm{IC}_{50}$ value of palmitic acid (C16:0) to palmitoleic acid (C16:1) a four-fold difference is observed (Table 4.5), which is very unusual as it is generally believed that unsaturation of the alkyl chains improves antibacterial activity [173-175].

When comparing the anti-mycobacterial activities of the fatty acids tested in combinations, the most effective mixtures were those that contain two fatty acids, where one of the fatty acids is palmitic acid (Table 4.5). When comparing activities of cis vs. trans FFAs it is hard to draw a conclusion, as only two fold differences were observed when comparing either $\mathrm{IC}_{50}$ of $\mathrm{MIC}_{90}$ values. Surprisingly, when trans $\mathrm{C} 18$ fatty acids were mixed with palmitic acid in a 1:1 ratio, roughly a six-fold improvement in activity was observed (see Table 4.5).

This result seems slightly unusual as it is generally believed that trans-fatty acids should display lower activity compared to cis-fatty acids. What seems more unusual is that the activities of the trans FFAs are enhanced by a saturated FFA [173-175]. The improvement in activity with palmitic acid is also observed in combination with palmitoleic acid, a C16 cis-fatty acid with a shift in $\mathrm{IC}_{50}$ from $274 \mu \mathrm{g} / \mathrm{mL}$ to $68 \mu \mathrm{g} / \mathrm{mL}$.

The data (Table 4.5) illustrates that palmitic acid, a C16 fatty acid, is more active compared to C18 fatty acids. Furthermore, based on these results, it seems that this saturated C16 fatty acid is more active than unsaturated fatty acids. Research studying the effects of FFAs on fast growing mycobacteria has identified that as chain length 
increased, activity decreased for saturated FFAs [171]. In addition, unsaturated FFAs tend to have better inhibitory activity with C16 chain lengths preferred over C18, however if $\mathrm{C} 18$ chains contained two double bonds the activity was comparable to that of the unsaturated C16 FFA [171]. As the aforementioned study focused on fast growing mycobacteria, a more relevant analogous comparison looking at slow growing mycobacteria identified a 2-4 fold improvement in MIC values for palmitic acid (C16) compared to unsaturated fatty (C18) acids [170].

Figure 4.1 illustrates the activity profile of the ND5_96L against stationary mycobacteria and mammalian cell lines. For the bacteriostatic assay, the highest concentration used was $250 \mu \mathrm{g} / \mathrm{mL}$ and at this concentration $90 \%$ inhibition of growth was observed. When analysing activity against stationary phase mycobacteria, ideally higher concentrations of drugs or samples should be used, as it is believed mycobacteria are more tolerant to inhibition by drugs in this state [195]. This can be illustrated in the drop in activity of streptomycin in a bacteriostatic assay (MIC $0.39 \mu \mathrm{M}$ ) compared to a stationary phase assay (MIC not achievable) (Appendix A). This assay showed that ND5_96L had an MIC90 of $500 \mu \mathrm{g} / \mathrm{mL}$ (Figure 4.1), which is two-fold greater when compared to the bacteriostatic assay. Studying the activity of the mixture in HL-60 and HEK cells identified the mixture was two-fold more active in HL-60 cells than HEK cells when comparing $\mathrm{MIC}_{90}$ values (Figure 4.2).

\subsubsection{Palmitic acid and mycobacteria}

Based on the activity of fatty acids analysed, it was observed that palmitic acid had the most prominent activity. This section highlights work relating to palmitic acid and mycobacteria. One of the earliest studies in this area identified growth inhibitory effects at higher concentrations of the fatty acid [196]. However, when non-toxic concentrations were used, the saturated acid was incorporated into cells. Following assimilation of all the palmitic acid within the growth medium, further doses of noninhibitory concentrations of palmitic acid could be added to the media, which was in turn consumed. This assimilation process was dependent on aerobic conditions [196].

Studying the effects of complexing fatty acids with detergent to reduce their toxicity identified that when Triton WR1339 was added in a 2.5:1 ratio, the toxicity of palmitic acid was reduced. This was due to the detergent sequestering the fatty acids, making less available to inhibit bacterial growth [197]. Testing the effects of fatty acids in the 
absence of the detergent against $M$. tuberculosis identified inhibition of growth by oleic and palmitic acids at $2 \mu \mathrm{g} / \mathrm{mL}$ [197]. This inhibitory concentration is inconsistent with the work described here and that of others as stated below. In our assay the MIC (an observed maximum of $2 \%$ growth) was $1 \mathrm{mg} / \mathrm{mL}$, with greater than $90 \%$ growth observed at the lowest tested concentration, $7.8 \mu \mathrm{g} / \mathrm{mL}$.

The effects of palmitic acid at non-inhibitory concentrations on the metabolism of M. avium identified that this fatty acid is incorporated to form lipids, a majority of them being triglycerides [198]. A follow-up study focusing on the effects of the fatty acid on cell division identified that the acid increased the formation of clear virulent colonies compared to the cultures starved of palmitic acid, which selected for opaque non-virulent colonies [199].

A study of the anti-mycobacterial component of the hexane extract of Humulus lupulus identified palmitic, stearic and oleic acids as the major components. The growth inhibitory effect of these fatty acids on $M$. fortuitum identified an MIC of $512 \mu \mathrm{g} / \mathrm{mL}$ for palmitic acid using a broth microdilution assay, however no activity was observed against $M$. smegmatis, $M$. phlei or $M$. aurum. In the same study oleic and linoleic acids proved more active with MIC's ranging from 4-16 $\mu \mathrm{g} / \mathrm{mL}$ [200]. The reported activity for palmitic acid in the same study is more consistent with the study described here. However, the activities of oleic and linoleic acids do not match the results of this thesis with more than $90 \%$ growth observed at concentrations ranging from $4-16 \mu \mathrm{g} / \mathrm{mL}$.

A similar study focusing on a native South African herbal remedy made from the root extracts of Pelargonium reniforme and $P$. sidoides identified in vitro activity from the hexanes fractions. Bioassay-guided fractionation identified that a mixture of fatty acids was responsible for this activity. Testing of the saturated and unsaturated components found an MIC of $256 \mu \mathrm{g} / \mathrm{mL}$ against $M$. aurum for palmitic acid, which was inactive against M. smegmatis, M. fortuitum, M. abscessus or M. phlei. This study also identified unsaturated fatty acids as being more active than saturated fatty acids. They reported oleic acid MIC's ranging from 4-256 $\mu \mathrm{g} / \mathrm{mL}$, linoleic acid MIC's ranging from 2-32 $\mu \mathrm{g} / \mathrm{mL}$ and palmitoleic acid with MIC's ranging from $4-16 \mu \mathrm{g} / \mathrm{mL}$ for the different species of mycobacteria [171]. 
Bioassay-guided isolation focusing on the anti-mycobacterial activity of a red alga, Polysiphonia virgata, identified a mixture of fatty acids from the dichloromethane extract as being responsible for activity. Studying the effect of individual fatty acids identified that the most active fatty acids were again unsaturated fatty acids with oleic acid having an MIC of $25 \mu \mathrm{g} / \mathrm{mL}$, and linoleic, myristic and lauric acids having MICs of $50 \mu \mathrm{g} / \mathrm{mL}$ against $M$. tuberculosis. Testing the acids against a strain of MDR-TB identified the same MIC values for linoleic, myristic and lauric acids, with oleic acid reported as inactive in this strain. This paper reported palmitic acid as inactive [201].

A more recent study on the bioactivity of the hexane extract of fruit peel of Citrus aurantifolia (Mexican lime) identified fatty acids as the components responsible for activity, with MICs in the range of $25-50 \mu \mathrm{g} / \mathrm{mL}$ for palmitic acid against M. tuberculosis $\mathrm{H} 37 \mathrm{Rv}$ and three other mono-resistant strains of TB. Interestingly, this paper reported that the unsaturated fatty acids linoleic and oleic acid were slightly less active with MICs ranging from 50-100 $\mu \mathrm{g} / \mathrm{mL}$. This study complements the results presented in this thesis, with the saturated acids identified as more active than the unsaturated, although the reported MIC for palmitic acid by the authors matches more closely to the observed $\mathrm{IC}_{50}$ of $66 \mu \mathrm{g} / \mathrm{mL}$ described in this thesis [170].

\subsubsection{General summary}

In general, current literature has suggested that unsaturated fatty acids tend to be more active than saturated ones. However, this work demonstrates that when looking at the activities of the fatty acids, palmitic acid, a C16 saturated fatty acid, displays greater activity than the $\mathrm{C} 16$ and $\mathrm{C} 18$ unsaturated fatty acids that have been tested.

Also worth noting is the current view that cis fatty acids tend to be more active than trans fatty acids. When comparing the activities of $c i s$-vaccenic to trans-vaccenic acids, this holds marginally true. However, the combinations of palmitic and trans-vaccenic acids gave the most effective $\mathrm{IC}_{50}$ value of all the different mixtures tested with a two-fold improvement in activity compared to the mixture of palmitic and cis-vaccenic acids.

One trend that emerges from the literature is that unsaturated fatty acids tend to be more active against mycobacteria [171, 200, 201]. Assessing the inhibitory effects of fatty acids on mycobacteria has identified that for saturated FFAs, once the chain length 
exceeds 14 carbons, the inhibitory effects decreases. This effect is overcome by introducing unsaturation within the chain [171].

There are conflicting views of the activity of palmitic acid against mycobacteria, nonetheless, this work identified fatty acids as moderate to weak inhibitors of mycobacteria, with palmitic acid causing the most inhibition.

When reviewing the literature, invertebrates tend to be the major producers of secondary metabolites, which are utilised as a form of self-defence. Reports of secondary metabolites being produced by marine vertebrates are very rare, this can be seen by the paucity of reports from comprehensive reviews [89, 202, 203]. The most commonly reported classes of natural products from marine vertebrates are peptides and steroids. The peptide toxins grammistins were isolated from the soap fish Grammistes sexlineatus [204]. From the dogfish shark Squalus acanthias, squalamine (41) and related aminosterols were identified, $\mathbf{4 1}$ is believed to be part of the host's innate defence and was identified to have activity against Gram-positive and Gram-negative bacteria [205, 206]. Related to $\mathbf{4 1}$, is petromyzonamine disulfate (42), which was isolated from a sea lamprey along with two other migratory pheromones [207].
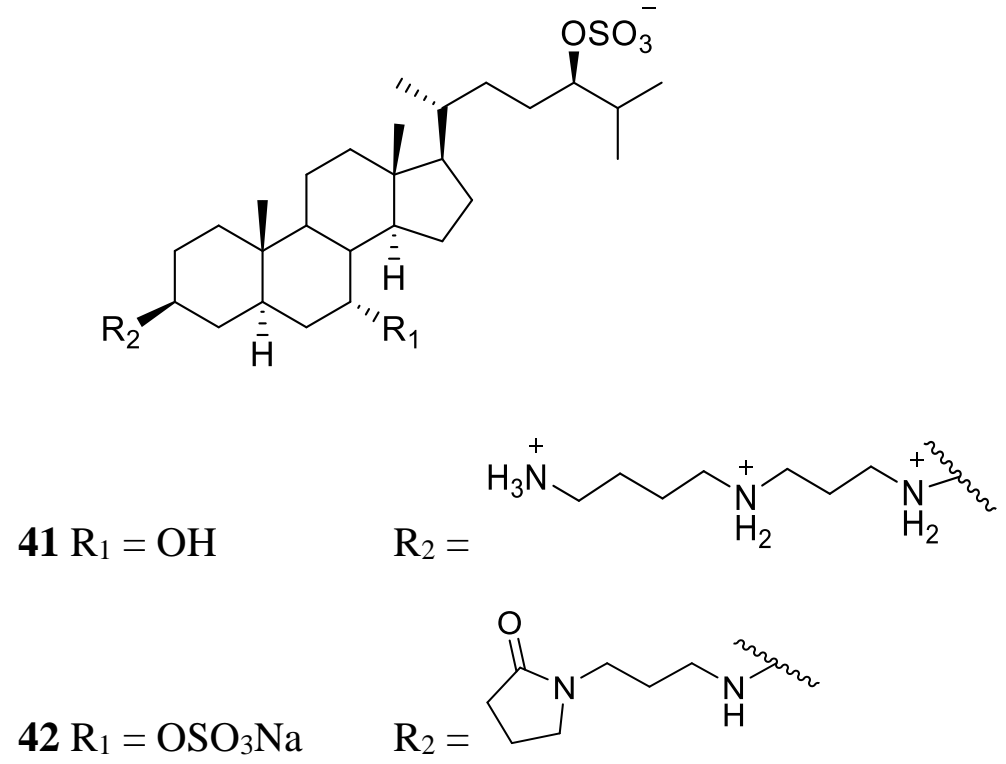

It is of no surprise therefore that the bioactive component of the blob fish liver extract was a mixture of primary metabolites, much like the examples of marine vertebrate derived compounds listed above. 


\section{Chapter 5 Rimarikiamide A from \\ Latrunculia sp.}




\subsection{Background}

Screening the VUW MNP library identified the crude extract of a Latrunculia sponge (PTN_256C) as an active inhibitor against M. smegmatis and S. aureus at the screening concentration of $100 \mu \mathrm{g} / \mathrm{mL}$. The subsequent validation of the crude extract in M. tuberculosis H37Ra found it to be more active in $\mathrm{H} 37 \mathrm{Ra}\left(\mathrm{MIC}_{90}=6.25 \mu \mathrm{g} / \mathrm{mL}\right)$, compared to $M$. smegmatis ( $\mathrm{MIC}_{90}=100 \mu \mathrm{g} / \mathrm{mL}$ ) (Table 3.3). Bioassay-guided fractionation of this sponge led to the identification of a novel taurinated terpene with activity against cancer cells in vitro, in addition to other fractions with antimycobacterial activity. This chapter describes the bioactivity, isolation and structure elucidation of the taurinated terpene. Furthermore this chapter gives an overview of taurinated marine natural products as well as known biological activities linked to the compounds.

Taurinated marine natural products are common as the presence of taurine in marine organisms is ubiquitous [208, 209]. The bioactivity reported for most of the compounds is generally anti-cancer due to the common use of anti-tumor assays in screening for novel compounds. Using SciFinder ${ }^{\circledR}$ and MarinLit [210], a literature search was performed to identify published work on taurinated marine natural products. From this search it was evident that sponges predominate as a major source of taurinated compounds. Most instances of taurinated marine natural compounds tend to involve taurine groups linked to a long alkyl chain or long fatty acids. The focus of this literature review will be on marine natural products that have a taurine group attached via an amine functionality. Compounds where taurine has been completely incorporated into the backbone of the compound will not be discussed. The compounds discussed below are grouped based on the common functionalities and carbon skeletons they share.

\subsubsection{Taurinated fatty acid derivatives}

The taurinated compounds grouped in this class are either conjugates of fatty acids and taurine, or a major portion of their structure is derived from a fatty acid. A recent report highlighted the isolation of 2-heptadec-11-enamidoethanesulfonic acid (43), from a South China Sea sponge Axinella sp. [211]. The authors also re-isolated two known compounds 2-octadec-7-enamidoethanesulfonic acid (44) and 2-palmitamidoethanesulf- 
onic acid (45). Compound 45 was first isolated from a South China sea sponge Haliclona sp. [212]; there has been no reported bioactivity for any of these compounds.<smiles>CCCCC/C=C/CCC(=O)NCCS(=O)(=O)O</smiles>

43

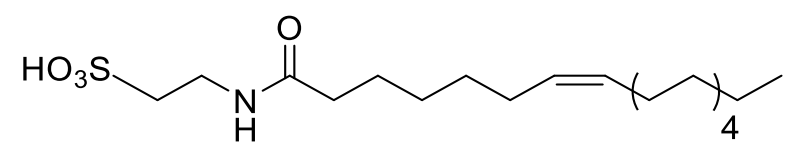

44<smiles>O=C(CCCCCCCCCS(=O)(=O)O)NCCS(=O)(=O)O</smiles>

45

Structurally related are two acylated taurine fatty acid derivatives, (all-Z-)-2-(icosa-5,8,11,14,17-pentaenoylamino)ethane sulfonic acid (46) from a star fish Certonardoa semiregularis, and 47 isolated from the sponge Erylus nobilis [213]. These two compounds were isolated guided by a brine shrimp lethality assay. An $N$-acyl taurine (48) was isolated from a sea urchin Glyptocidaris crenularis with a reported $\mathrm{LD}_{50}$ of $222 \mu \mathrm{g} / \mathrm{mL}$ in a brine shrimp assay [214].

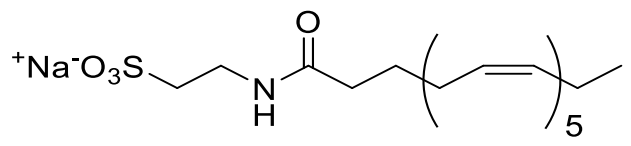

46<smiles>CC(C)CCCC/C=C/C=CCC(=O)NCCSO[Na]</smiles> 


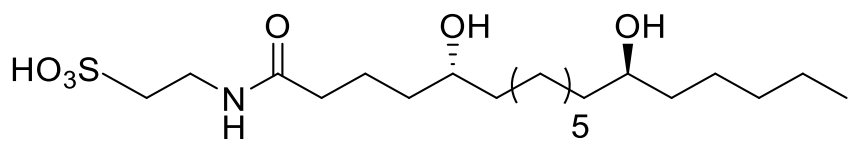

48

Irciniasulfonic acid $\mathrm{B}_{1}(\mathbf{4 9})$ and $\mathrm{B}_{2}(\mathbf{5 0})$, a mixture of two taurinated fatty acids isolated from a Japanese marine sponge Ircinia sp., restored sensitivity to vincristine in resistant cancer cells [215]. Taurolipids A (51), B (52), and C (53), isolated from the protozoa Tetrahymena [216], have been reported to inhibit Clostridium perfringens sialidase enzyme (20 mU) by $50 \%$ at 140 and 110 pM for $\mathbf{5 1}$ and $\mathbf{5 2}$, respectively [217]. This enzyme cleaves sialic acid from the cell surface and glycoproteins and has been implicated in virulence of bacteria [218].<smiles>[R]OC(CC)CCCC/C(C)=C\C(=O)NCCS(=O)(=O)O</smiles><smiles>CCCCC(C)CC=CCCC=CCCCC(C)=O</smiles><smiles>CCCCCC/C=C/CCCC(C)=O</smiles><smiles>[R]C(CCCCC(O)CCCC(OC(=O)NCCCOS(=O)(=O)O)C([R2])C(=O)NCCCCCC)C(O)CCCC</smiles>

$$
51 \mathrm{R}_{1}=\mathrm{R}_{2}=\mathrm{H} \quad \mathbf{5 2} \mathrm{R}_{1}=\mathrm{H}, \mathrm{R}_{2}=\mathrm{OH} \quad 53 \mathrm{R}_{1}=\mathrm{R}_{2}=\mathrm{OH}
$$

Taurospongin A (54), an acetylenic lipid isolated from the Okinawan sponge Hippospongia sp., was shown to inhibit DNA polymerase $\beta$ and human immunodeficiency virus (HIV) reverse transcriptase [219]. Under normal settings, DNA 
polymerase $\beta$ plays a role in base excision repair, however, overexpression of variants of this enzyme have been linked to cancer [220].

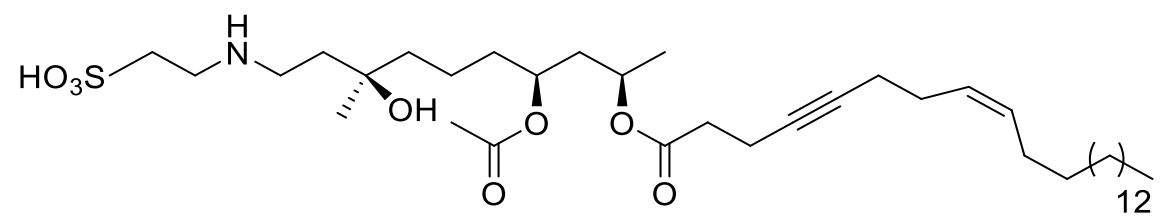

54

Carteriosulfonic acids A-C (55-57), isolated from a Carteriospongia sp. sponge collected in the Philippines, were shown to inhibit the kinase GSK-3 $\beta$, which is part of the Wnt signalling pathway. This pathway is involved in several human diseases including cancer [221, 222].<smiles>[R6]C(C)C[C@H](O)[C@@H](O)C[C@H](O)CCC(=O)NCC[Sb](=O)(=O)[O-]</smiles>

$55 \mathrm{R}=$

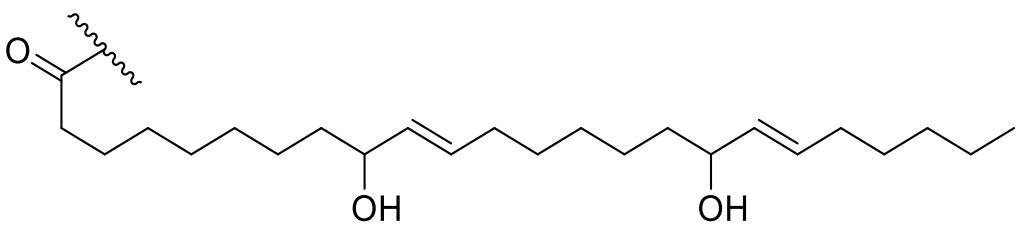

$56 \mathrm{R}=$
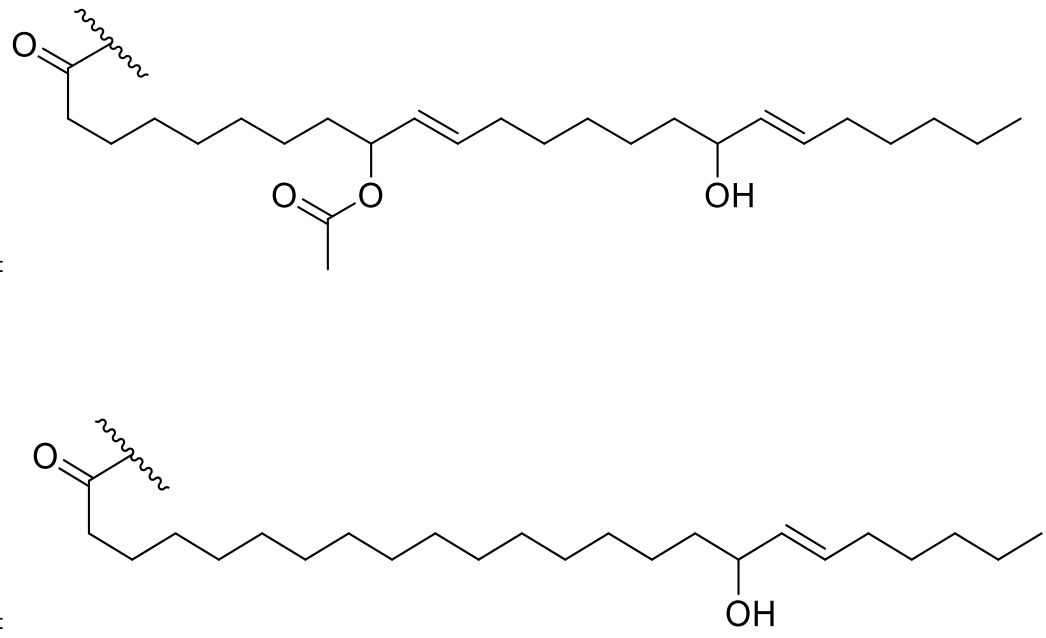
Tauro-pinnaic acid B (58), a novel taurinated fatty acid, was isolated from the Okinawan bivalve Pinna muricata. This compound displayed an $\mathrm{IC}_{50}$ of $90 \mu \mathrm{M}$ against cytosolic phospholipase A2, in a search for potential anti-inflammatory compounds [223].

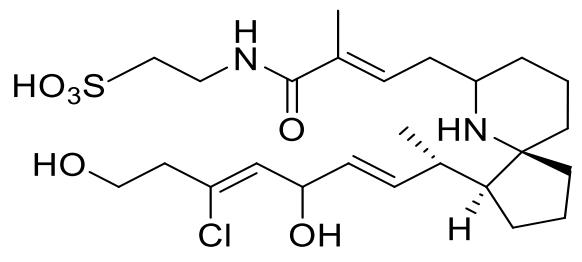

58

\subsubsection{Bromopyrrole containing compounds}

This next set of seven taurinated compounds all contain a bromopyrrole functionality, with all but one compound also featuring guanidine. Tauroacidins A (59) and B (60), isolated from the Okinawan sponge Hymeniacidon sp., displayed inhibitory activity against EGF receptor kinase and c-erbB-2 kinase each with IC $_{50}$ values of $20 \mu \mathrm{g} / \mathrm{mL}$ [224]. The increased activity or overexpression of these kinases has been linked to different types of cancers [225].<smiles>N=C1N=C(NCCS(=O)(=O)O)/C(=C/C(O)CNC(=O)c2cc(Br)c(Br)[nH]2)N1</smiles>

59<smiles>N=C1N=C(NCCS(=O)(=O)O)/C(=C/C(O)CNC(=O)c2cc(Br)c[nH]2)N1</smiles>

60

Mauritamide A (61), was isolated from the Fijian sponge Agelas mauritiana [226], and congeners mauritamide B-D (62-64) were isolated from the Indonesian sponge A. linnaei [227]. Mauritamide D (64) lacks the commonly found guanidine moiety. None of the mauritamides have any bioactivity reported to date. 
<smiles>COS(=O)(=O)CCNC1(CCCNC(=O)c2cc(Br)c(Br)n2C)NC(=N)NC1=O</smiles>

61<smiles>Cn1c(C(=O)NCCCC2(NCCS(=O)(=O)O)NC(=N)NC2=O)cc(Br)c1Br</smiles>

62<smiles>CCN1C(=N)NC(=O)C1(CCCNC(=O)c1cc(Br)c(Br)n1C)NCCS(=O)(=O)O</smiles>

63<smiles>Cn1c(C(=O)NCCS(=O)(=O)O)cc(Br)c1Br</smiles>

64

Taurodispacamide A (65), isolated from a Mediterranean sponge A. oroides, displayed anti-histamine activity when tested in the guinea pig ileum assay [228]. Nagelamide $\mathrm{H}$ (66), isolated from an Agelas sp., exhibited antibacterial activity against Micrococcus luteus and Bacillus subtilis with respective MIC's of 16.7 and $33.3 \mu \mathrm{g} / \mathrm{mL}$, in addition an $\mathrm{IC}_{50}$ of $46 \mu \mathrm{M}$ against protein phosphatase $2 \mathrm{~A}$ was also reported [229].<smiles>N=[14C]1NC(=NCCS(=O)(=O)[O-])/C(=C\CCNC(=O)c2cc(Br)c(Br)[nH]2)N1</smiles>

65<smiles>N=C1NC(=NCCS(=O)(=O)O[Na])C(/C=C/CNC(=O)c2cc(Br)c(Br)[nH]2)(/C=C/C2=C(C(=O)c3cc(Br)c(Br)[nH]3)NC(N)N2)N1</smiles>

66

\subsubsection{Aromatic compounds}

The taurinated compounds listed in this section contain aromatic functionalities as part of their structure. 2-[(Phenylacetyl)]aminoethanesulfonic acid (67), a conjugate of phenylacetic acid and taurine, was isolated from Colobometra perspinosa, an Australian crinoid, with a reported $\mathrm{GI}_{50}$ of $195 \mu \mathrm{M}$ against the MCF-7 breast-pleural effusion 
adenocarcinoma cell line [230]. Pyridinebetaine B (68) was isolated from a Caribbean sponge, Agelas dispar [231].<smiles>O=C(Cc1ccccc1)NCCS(=O)(=O)O</smiles>

67<smiles>O=S(=O)(O)CC[n+]1ccccc1</smiles>

68

Secoadociaquinones A (69) and B (70), isolated from a Xestospongia sp. sponge from the Philippines, showed inhibitory activity against topoisomerase II [232]. This enzyme creates double strand breaks when uncoiling DNA during replication. Consequently, its activity is more prominent in actively replicating cells, such as cancer cells. As such, many cancer therapeutics target this enzyme [233].<smiles>C[C@]12CCCc3coc(c31)C(=O)c1cc3c(cc12)C(=O)C(NCCS(=O)(=O)O)=CC3=O</smiles>

69<smiles>C[C@]12CCCc3coc(c31)C(=O)c1cc3c(cc12)C(=O)C(NCCS(=O)(=O)O)=CC3=O</smiles>

70

Cribrostatin 7 (71), a novel taurinated isoquinoline derivative with an $\mathrm{IC}_{50}$ of $45 \mu \mathrm{g} / \mathrm{mL}$ against human colon carcinoma (HCT 116) cells, was reported from a Petrosia sp. sponge, also collected in the Philippines [234].<smiles>C/C=C(/C)C(=O)OCc1nccc2c1C(=O)C(NCCS(=O)(=O)O)=C(C)C2=O</smiles> 
The heteroaromatic alkaloids nakijiamine $C(\mathbf{7 2})$ and $D(\mathbf{7 3})$, were isolated from an Okinawan sponge Suberites sp., with no reported bioactivity from these compounds [235]. Microxine (74), a taurinated purine derivative isolated from an Australian sponge Microxina sp., was reported to have inhibitory activity against cdc2 kinase with an $\mathrm{IC}_{50}$ of $13 \mu \mathrm{M}$ [236]. This enzyme is involved in the cell cycle and its entry into mitosis [237].<smiles></smiles>

72<smiles>C[N+]1(C)CC(c2c(O)c(NC(=O)CS(C)(C)C)c3nccc4c3c2C=CN4)c2c[nH]c3cc(Br)cc1c23</smiles>

73<smiles>Cn1c(=O)[nH]c2ncnc(NCCS(=O)(=O)O)c21</smiles>

74

A trichostatin analogue, JBIR-110 (75) was reported from the marine sponge derived Streptomyces sp. strain RM72, with a reported $\mathrm{IC}_{50}$ value against HDAC1 of $74 \mu \mathrm{M}$ [238]. HDACs are involved in epigenetic activity; the enzyme is involved in condensing chromatin and therefore altering gene expression [239]. These types of inhibitors have been studied as potential anti-cancer agents [239]. From the sponge Leucetta microhaphis, the alkaloid (9E)-clathridine 9- $N$-(2-sulfoethyl) imine (76), was isolated [240]. 
<smiles>CC(/C=C/C(=O)NCCS(=O)(=O)O)=C\[C@@H](C)C(=O)c1ccc(N(C)C)cc1</smiles>

75

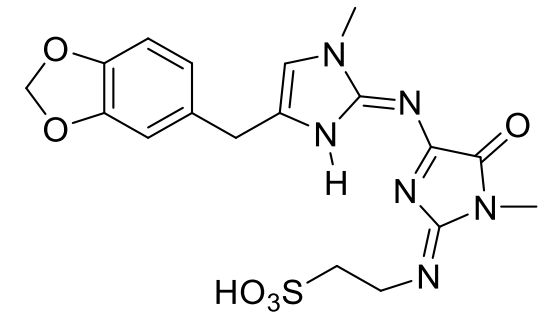

76

\subsubsection{Miscellaneous taurinated compounds}

Mycosporine-taurine (77) was isolated from the sea anemone Anthopleura elegantissma in a study targetting compounds responsible for providing UV protection [241]. From the Okinawan ascidian Didemnidae sp., a novel chlorinated polyketide macrolide biselide D (78) was reported [242].<smiles>COC1=C(NCCS(=O)(=O)O)CC(O)(CO)CC1=O</smiles>

77<smiles>C/C1=C/C/C=C(/Cl)CCC2C[C@@H](OC(=O)C/C=C(\O)CC(=O)NCCS(=O)(=O)O)O[C@@H]2C1</smiles>

78

\subsubsection{Taurinated terpenoids}

This section highlights taurinated terpenes. Terpenes are linear or cyclised hydrocarbon chains comprised of repeating isoprene units. (+)-4 $\beta-N$-Methenetauryl-10 $\beta$-methoxy$1 \beta, 5 \alpha, 6 \beta, 7 \beta$-aromadendrane (79) and (-)-4 $\beta-N$-methenetauryl-10 $\beta$-methoxy-1 $\beta, 5 \beta, 6 \alpha, 7$ $\alpha$-aromadendrane $(\mathbf{8 0})$, isolated from a South China Sea gorgonian coral Melitodes squamata, displayed inhibitory activity against $B$. subtilis and M. luteus at 
$25 \mu \mathrm{g}$ in a disk diffusion assay [243]. The bicyclic terpenoids, clathrimides A (81) and B (82), isolated from the sponge Clathria compressa [244], showed no activity against human embryonic stem cells (BG02), Gram-positive (Staphylococcus aureus including methicillin-resistant S.aureus) or Gram-negative (Escherichia coli and Klebsiella pneumonia) bacteria.

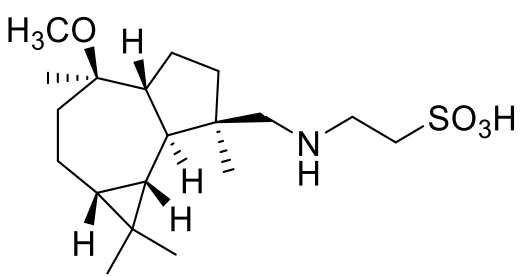

79

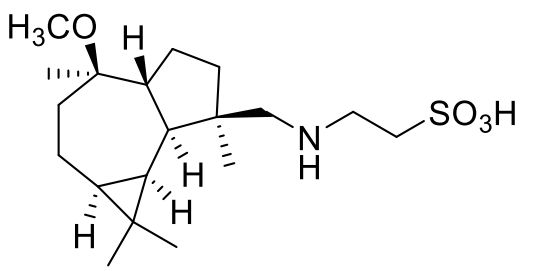

80<smiles>[R]C1(C)CCC[C@]2(C)[C@H](CC(=O)NCCS(=O)(=O)O)C(=C)CC[C@H]12</smiles><smiles>CC(C=[W])=C/C=C\C(C)C</smiles>

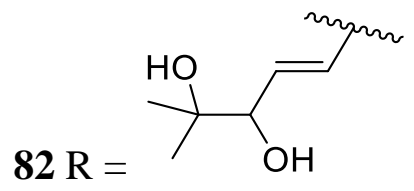

Spongodine D $(\mathbf{8 3})$, contains a pyridine functionality with inhibitory activity against human synovial phospholipase A2 (PLA2), and was isolated from a Vanuatu sponge Spongia sp. [245]. PLA2 is responsible for the release of free fatty acids, which are then metabolised to produce pro-inflammatory mediators [246]. Phorbasin D (84), a diterpene-taurine conjugate, was isolated from the Phorbas sp., Australian sponge. This compound was tested and reported as inactive against lung (A549) and breast (HT29 and MDA-MB-231) cancer cell lines [247]. 


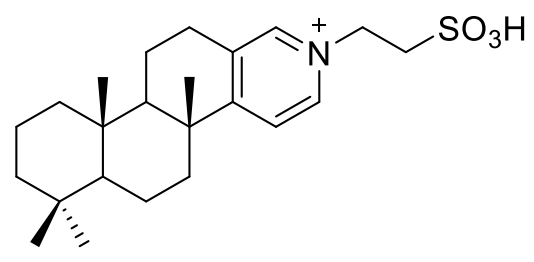

83<smiles>C/C(=C\C=C\C(C)C=CCC(C)C)[C@H]1[C@@H](O)C=C(CNCCS(=O)(=O)O)C(=O)[C@H]1O</smiles>

Gukulenin E (85), a diterpene pseudo-dimer, was isolated from Phorbas gukulensis, a Korean sponge, with a reported $\mathrm{LC}_{50}$ of 0.32 and $0.11 \mu \mathrm{M}$ against $\mathrm{K} 562$ and $\mathrm{A} 549$ cell lines, respectively [248].

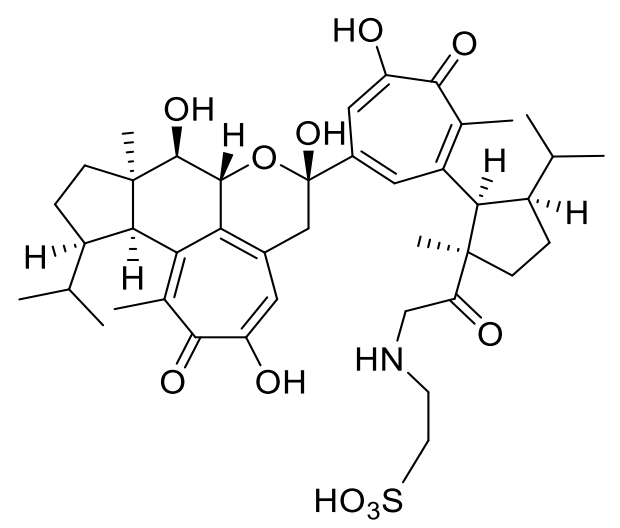

85

\subsection{Meroterpenes}

Meroterpenes are compounds that are of mixed biogenesis and contain a terpene attached to an aromatic functionality. Aplidone C (86) was isolated from the Mediterranean ascidian Aplidium conicum and had no reported activity [249]. Siphonodictyal B1 (87) was isolated from a Caribbean sponge, Aka coralliphagum, with reported inhibitory activity against $S$. aureus at $12 \mu \mathrm{g} / \mathrm{mL}$ and cytotoxic activity against L929 mouse fibroblasts at $10 \mu \mathrm{g} / \mathrm{mL}$ [250]. Nakijiquinone $\mathrm{R}$ (88), a 
sesquiterpenoid quinone, was isolated from an Okinawan sponge belonging to the family Spongiidae. This compound displayed greater than $99 \%$ and 52\% inhibition against EGFR and HER2 kinases at $1 \mathrm{mM}$, respectively [251].

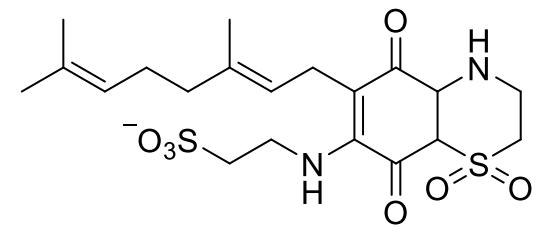

86

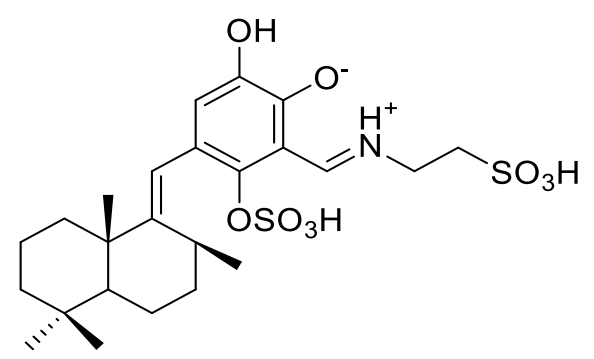

87

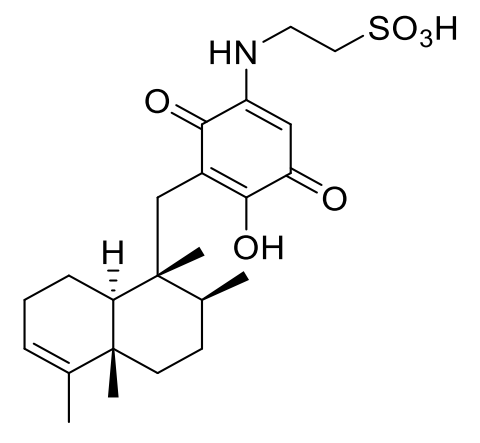

88

Melemeleones A (89) and B (90) were isolated from the Solomon Islands sponge Dysidea avara. Melemeleone B displayed an approximate $\mathrm{IC}_{50}$ of $28 \mu \mathrm{M}$ in an assay against the $\mathrm{pp}^{\mathrm{v}} \mathrm{v}$-src $\mathrm{PTK}$, a tyrosine kinase [252]. Tyrosine kinase inhibitors are important in the treatment of cancer progression [253]. An anti-inflammatory sequiterpene dysidine (91), was isolated from a Dysidea sponge from Vanuatu. This compound was shown to inhibit the activity of human synovial PLA2, with an $\mathrm{IC}_{50}$ of $2.0 \mu \mathrm{M}$ [254]. 


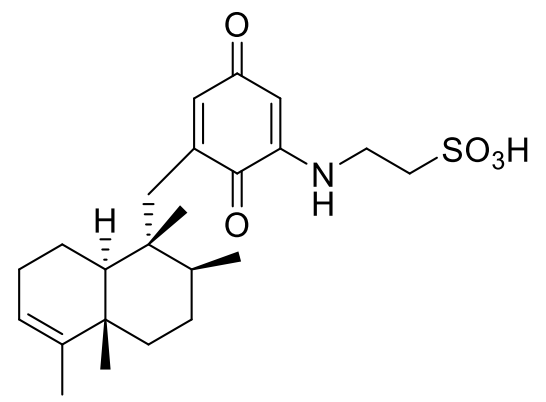

89

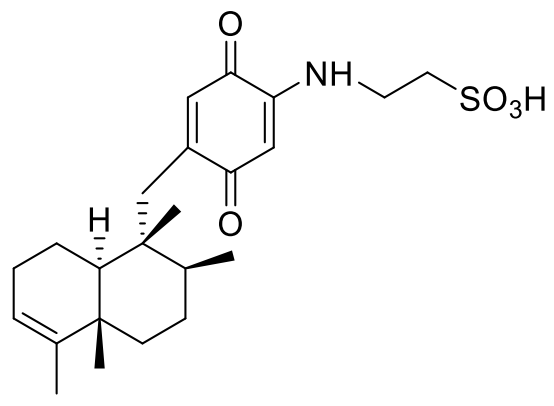

90<smiles>CC1=CCC[C@H]2[C@@H](C)[C@](C)(CC3=C(O)C(=O)C=C(NCCS(=O)(=O)O)C3=O)CC[C@]12C</smiles>

91

\subsubsection{Taurinated sterols}

The next set of six compounds are taurinated sterols, which have all been isolated from starfish. From Myxoderma platyacanthum, (92) and (93) were isolated [255]. $(20 R, 22 E, 24 R, 25 S)$-24-Methyl-3 $\beta, 6 \alpha, 8,15 \beta$-tetrahydroxy-5 $\alpha$-cholest-22-en-27-oic acid (94), was isolated from Astropecten triseriatus [256]. Minutoside B (95), isolated from Anasterias minuta, had inhibitory activity against Aspergillus flavus at concentrations between 20-60 $\mu \mathrm{g} / \mathrm{mL}$ in a disk diffusion assay [257]. Novaeguinosides B (96) and D (97), were isolated from Culcita novaeguineae, with reported $\mathrm{IC}_{50}$ values of 7.9 and 9.5 $\mu \mathrm{M}$ against K-563 and BEL-7402 cell lines for 96 and 4.6 and $4.1 \mu \mathrm{M}$ for 97 [258]. 


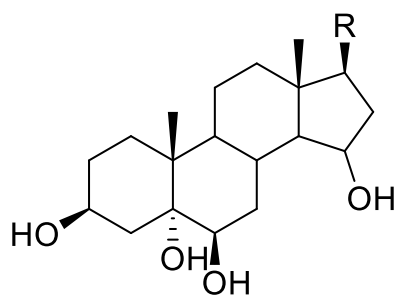

$92 \mathrm{R}={ }_{2}$

$93 \mathrm{R}={ }^{2}$ 


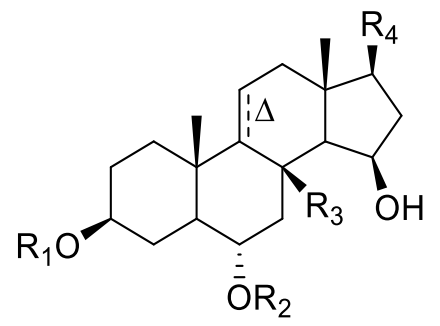

$94 \quad \mathrm{R}_{1}=\mathrm{R}_{2}=\mathrm{H} \mathrm{R}_{3}=\mathrm{OH}, \Delta$ sat.

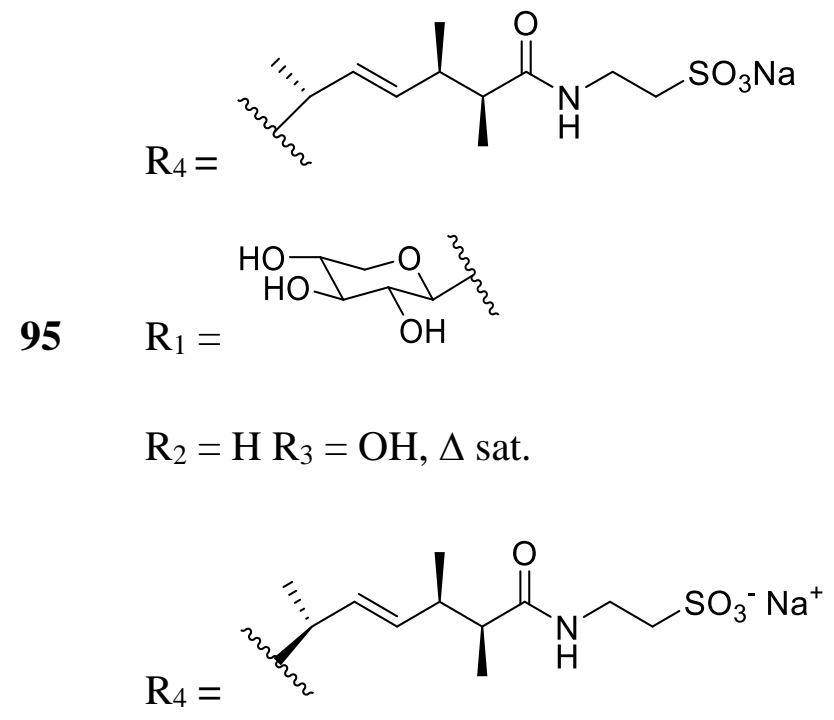

$96 \quad \mathrm{R}_{1}=\mathrm{SO}_{3} \mathrm{Na}$

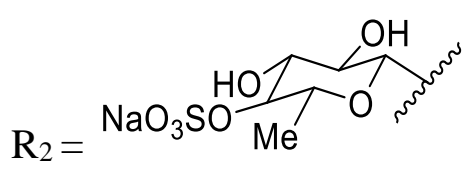

$\mathrm{R}_{3}=\mathrm{H}, \Delta$

$\mathrm{R}_{4}=\overbrace{m_{2}}$ 


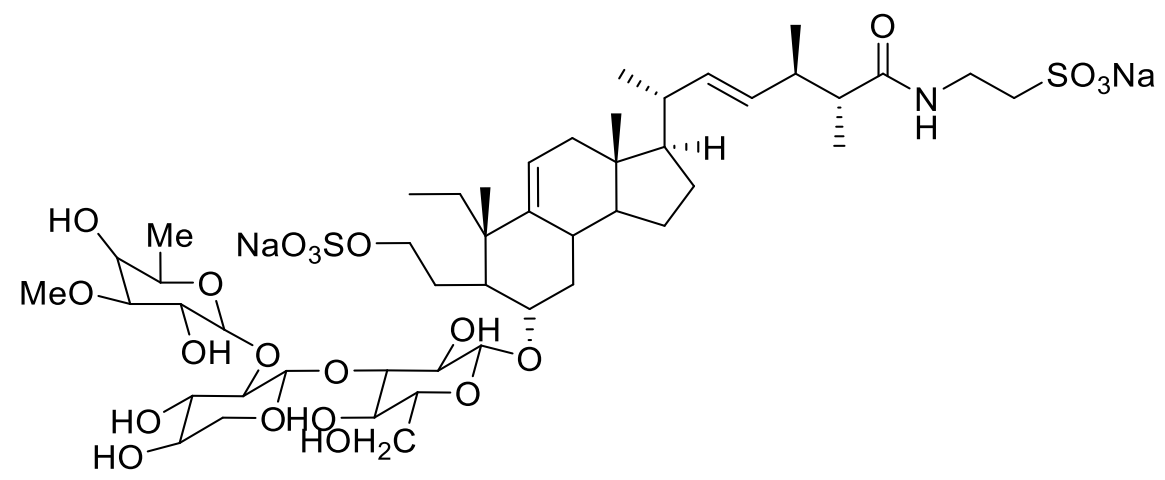




\subsection{Methods}

\subsubsection{Extraction of sponge material}

Latrunculia sp. (350 g), was cut into $\sim 1 \mathrm{~cm}^{3}$ sized pieces and extracted twice in methanol $(450 \mathrm{~mL})$. The two extracts were treated separately and filtered using a Büchner funnel and Celite. The second extract was passed through an HP20 column $(140 \mathrm{~mL})$ followed by the first. The eluents were combined and diluted two-fold with $\mathrm{H}_{2} \mathrm{O}$ before passing through the same column again. This was repeated until the original $\mathrm{MeOH}$ extract was diluted to $12.5 \%$ and passed through the column. Following this cyclic loading, the column was eluted with $420 \mathrm{~mL}$ volumes of $20 \%, 40 \%, 60 \%, 80 \%$ and $100 \%$ acetone in water. The $40 \%$ fraction was further fractionated using a $15 \mathrm{~mL}$ column of HP20ss resin, the column was eluted with $45 \mathrm{~mL}$ volumes of $50 \%, 60 \%$, $70 \%$, and $80 \% \mathrm{MeOH}$ and $\mathrm{H}_{2} \mathrm{O}$ solvent mixtures as well as $100 \% \mathrm{MeOH}$ and $100 \%$ acetone. Following this, the $80 \% \mathrm{MeOH} / \mathrm{H}_{2} \mathrm{O}$ fraction was further purified using reversed-phase C18 semi-preparative column (Phenomenex Prodigy, 10.0 x 250 mm, 10 $\mu \mathrm{m}$ particle size), $35 \%$ acetonitrile $/ \mathrm{H}_{2} \mathrm{O}$ at $4 \mathrm{~mL} / \mathrm{min}$. From the resulting fractions, ND4_34D and E were pooled together to give rimarikiamide A (98), (200 $\mu \mathrm{g})$ (Scheme 5.1). Quantification of samples lower than $1 \mathrm{mg}$ was performed as described in section 2.7 .

Based on this initial isolation, a repeat isolation was attempted, using the remaining sponge sample. As the structure of rimarikiamide A had already been elucidated from the first isolation, it was evident that the lack of a chromaphore would make a repeat isolation via HPLC problematic. Therefore size exclusion chromatography using LH20 was attempted using a $100 \mathrm{~mL}$ column, eluting with $100 \% \mathrm{MeOH}$ to give rimarikiamide A (98) $(133.7 \mu \mathrm{g})$. 


\subsubsection{Isolation schemes for rimarikiamide $A$}

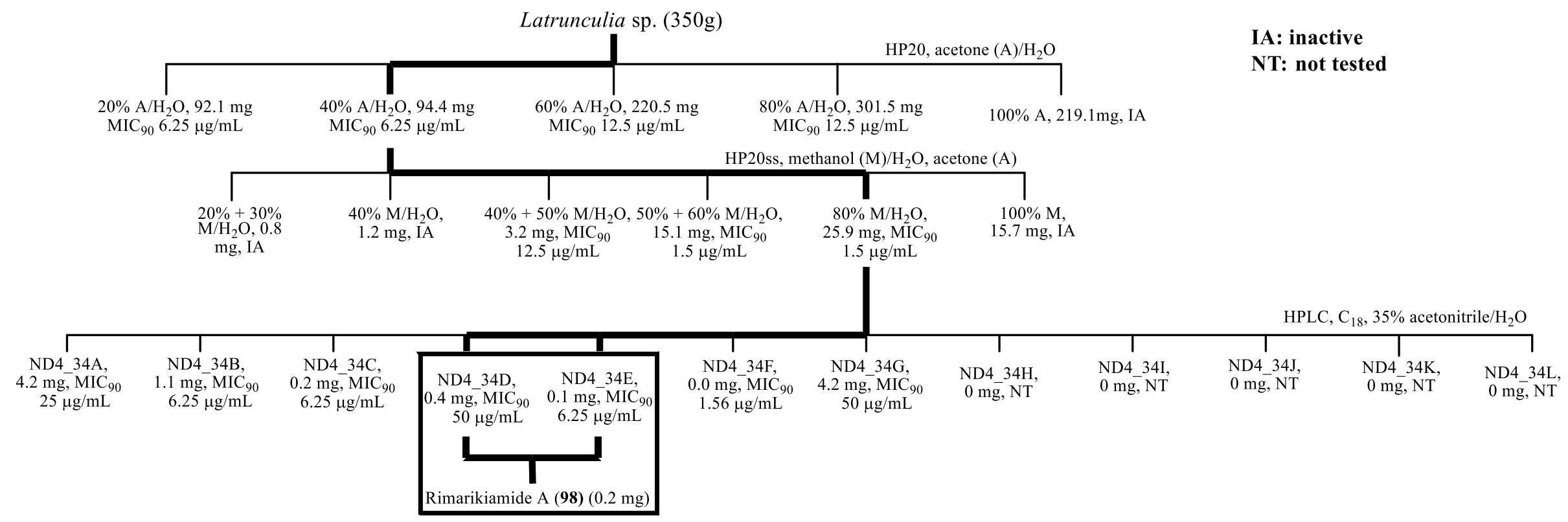

Scheme 5.1 Original isolation scheme for rimarikiamide A (98) from Latrunculia sp. 


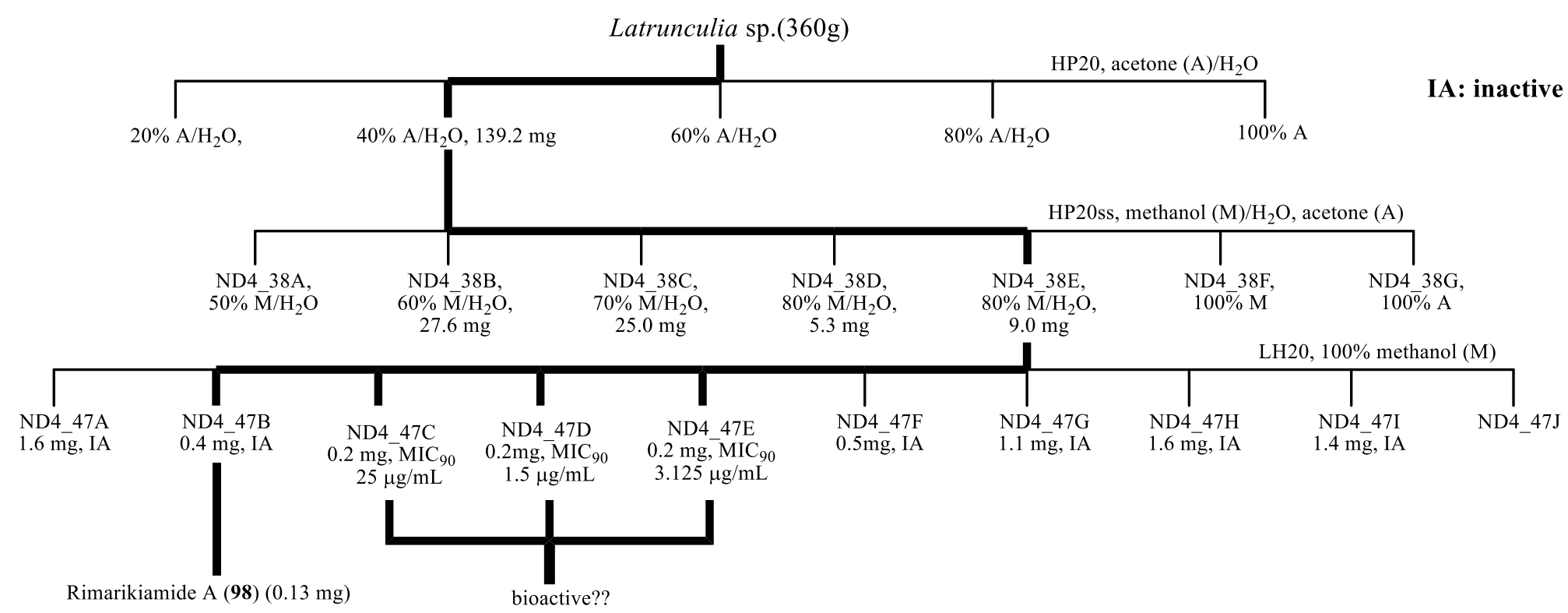

Scheme 5.2 Optimised isolation scheme for rimarikiamide A (98) from Latrunculia sp. 


\subsection{Results}

Using the methodology described in section 2.6, the results of the dose-response assays for rimarikiamide A are listed below. Graphs were generated using GraphPad Prism v.5.0. Resazurin dye was used to measure bacterial metabolism after each assay was run and RFU measurements were used as a measure of growth. OD was used as measure of growth for the HL-60 and HEK MTT assays. Where possible $\mathrm{IC}_{50}$ values have been listed, however as sigmoidal curves were not achievable for all data $\mathrm{MIC}_{90}$ values have been listed. 


\subsubsection{Antimicrobial activity}

Rimarikiamide A vs M. tuberculosis H37Ra

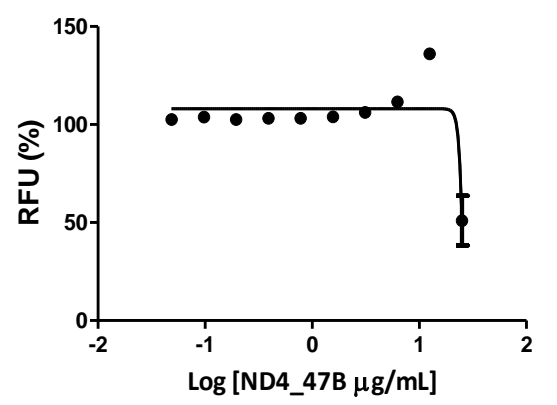

A

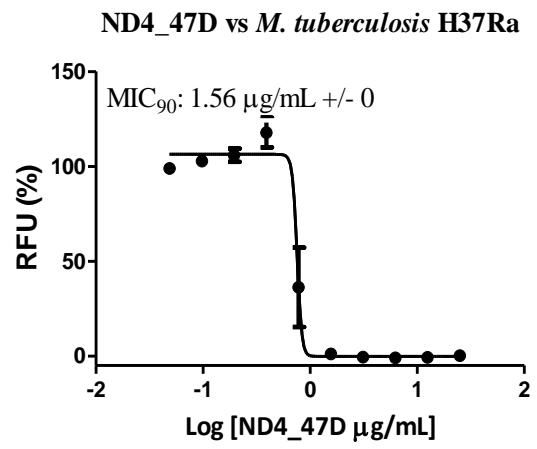

B

Figure 5.1 Loss of anti-mycobacterial activity of rimarikiamide A

(A), dose response curve illustrating the loss of activity of rimarikiamide A. (B), dose response curve of the active fraction ND4_47D. RFU units were used as a measure of growth.

$(\mathrm{n}=1$, duplicate $)$

\subsubsection{Mammalian cell line assays}

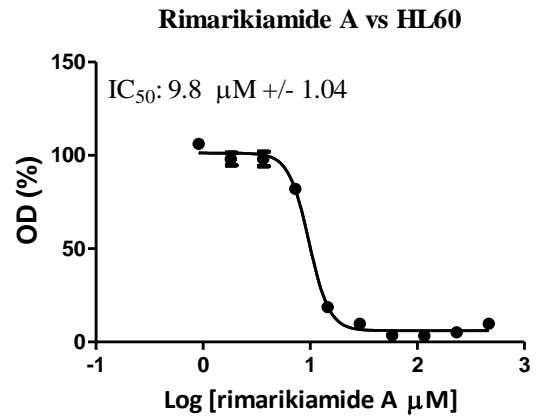

A

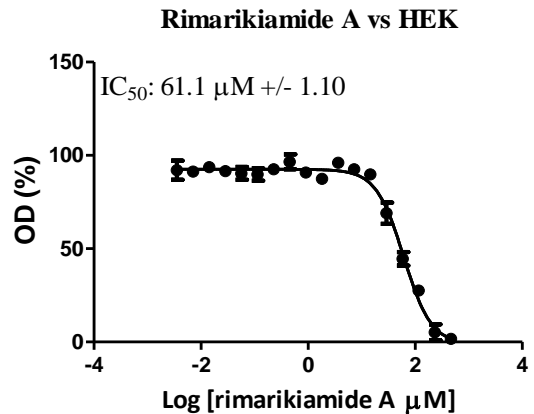

B

Figure 5.2 Rimarikiamide A vs human cell lines

MTT assay of ND6_96L against HL-60 (A) and HEK cell lines (B). The HEK cell line was used as an indication of general cytotoxicity. OD was used as a measure of growth. $(\mathrm{n}=1$, duplicate $)$ 


\subsection{Discussion}

\subsubsection{Fractionation and isolation}

Guided by bioassays against M. tuberculosis H37Ra, an initial crude fractionation using HP20 (PSDVB) column chromatography was performed, with the $40 \%$ acetone/ $\mathrm{H}_{2} \mathrm{O}$ fraction taken to the next stage of purification (Scheme 5.1). HP20ss (PSDVB) column chromatography was used to further purify this fraction culminating with reversedphase C18 semi-preparative HPLC to purify the final bioactive fraction rimarikiamide A $(200 \mu \mathrm{g})($ Scheme 5.1).

To isolate more compound, the second half of the sponge was extracted and fractionated to the point of collecting the $\mathrm{HP} 20 \mathrm{ss} 80 \% \mathrm{MeOH} / \mathrm{H}_{2} \mathrm{O}$ fraction (Scheme 5.2). As we could not rely on HPLC to guarantee the isolation of the compound due to the lack of a suitable chromophore, LH20 size exclusion chromatography was employed for the next stage of purification. Following size exclusion chromatography, the fractions of the second work up of the original sponge were combined based on TLC, and tested for activity (Scheme 5.2). This identified that the taurinated-compound initially believed to be responsible for activity was not the source of anti-mycobaterial activity, which can be observed when comparing the dose response curve of rimarikiamide A (98) to ND4_47D (Figure 5.1).

\subsubsection{Structural elucidation}

Negative ion HRESIMS was used to detect an [M-H] $]^{-}$ion at $m / z$ 428.2469, which was suitable for the molecular formula $\mathrm{C}_{22} \mathrm{H}_{39} \mathrm{NO}_{5} \mathrm{~S}$ (calcd $\mathrm{m} / \mathrm{z} 428.2471, \Delta=0.0002$ ), requiring four double bond equivalents. Both ${ }^{1} \mathrm{H}$ and multiplicity edited HSQC NMR spectra in $\mathrm{d}_{6}$-DMSO revealed that 36 of the 39 protons were attached to 18 carbons, including five methines $\left(\delta_{C} 124.5,124.2,121.3,76.0,29.8\right)$, eight methylenes $\left(\delta_{C} 50.7\right.$, $43.2,39.3,36.3,35.5,33.9,26.2,24.5)$, and five methyls ( $\delta_{\mathrm{C}} 25.6,19.4,17.6,16.1$, 11.3). The remaining four carbons in the molecule were quaternary carbons, which included an ester or amide carbonyl $\left(\delta_{\mathrm{C}} 171.0\right)$ and three olefinic carbons $\left(\delta_{\mathrm{C}} 137.4\right.$, 134.9, 130.6). The three remaining protons were therefore exchangeable. The presence of an ester or amide and three olefinic methines accounted for all the double bond equivalents required by the molecular formula, therefore the molecule must be linear. 
COSY and HMBC correlations established the connectivity for most of the structure. The terminal methyls $\left(\mathrm{CH}_{3}-16: \delta_{\mathrm{C}} 25.6, \delta_{\mathrm{H}} 1.63\right) ;\left(\mathrm{CH}_{3}-20: \delta_{\mathrm{C}} 17.6, \delta_{\mathrm{H}} 1.55\right)$ showed HMBC correlations to the olefinic quaternary carbon $\mathrm{C}-15\left(\delta_{\mathrm{C}} 130.6\right)$ and its neighbouring olefinic methine $\mathrm{C}-14\left(\delta_{\mathrm{C}} 124.2, \delta_{\mathrm{H}} 5.06\right)$. This was further supported by allylic COSY correlations from $\mathrm{H}_{3}-16$ and $\mathrm{H}_{3}-20$ to $\mathrm{H}-14$. $\mathrm{H}-14$ in turn showed a COSY correlation to the methylene proton resonances of $\mathrm{C}-13\left(\delta_{\mathrm{C}} 26.2, \delta_{\mathrm{H}} 2.00\right)$, which in turn correlated to methylene $\mathrm{C}-12\left(\delta_{\mathrm{C}} 39.3, \delta_{\mathrm{H}} 1.91\right)$. This established the first substructure below (Figure 5.3).

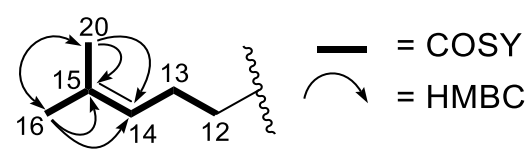

\section{Figure 5.3 Key COSY and HMBC correlations establishing substructure 1}

HMBC correlations from the methyl $\mathrm{CH}_{3}-19\left(\delta_{\mathrm{C}} 16.1, \delta_{\mathrm{H}} 1.54\right)$ to the olefinic methine $\mathrm{C}-10\left(\delta_{\mathrm{C}} 121.3, \delta_{\mathrm{H}} 5.04\right)$, quaternary $\mathrm{C}-11\left(\delta_{\mathrm{C}} 134.9\right)$ and methylene $\mathrm{C}-12$ helped further extend the substructure. This was further supported by HMBC correlations from C-12 to C-10, C-11 and C-19. COSY correlations observed from $\mathrm{H}-10$ to $\mathrm{H}_{2}-9\left(\delta_{\mathrm{C}} 33.9\right.$, $\left.\delta_{\mathrm{H}} 2.08\right), \mathrm{H}_{2}-9$ to the $\mathrm{C}-8$ methine $\left(\delta_{\mathrm{C}} 76.0 \delta_{\mathrm{H}} 3.77\right)$ and finally an allylic COSY correlation from $\mathrm{H}_{2}-12$ to the olefinic methine $\mathrm{H}-10$ helped extend the substructure (Figure 5.4).

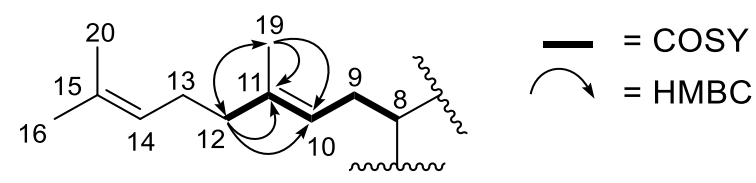

\section{Figure 5.4 Key COSY and HMBC correlations establishing substructure 2}

The chemical shifts of the C-8 methine $\left(\delta_{C} 76.0, \delta 3.77\right)$ were characteristic of a carbinol centre. Fortuitously a COSY correlation from H-8 to an exchangeable proton of an alcohol group $\left(\delta_{\mathrm{H}} 4.59\right)$ further supported the presence of this functional group (Figure 5.5).

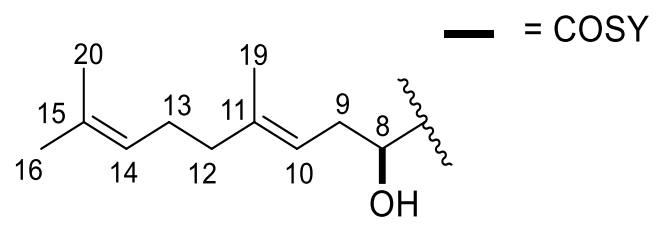


Figure 5.5 Key COSY and HMBC correlations establishing substructure 3

The substructure was extended by $\mathrm{HMBC}$ correlations from methyl $\mathrm{CH}_{3}-18\left(\delta_{\mathrm{C}} 11.3\right.$ $\left.\delta_{\mathrm{H}} 1.51\right)$, to $\mathrm{C}-8$, quaternary alkene $\mathrm{C}-7\left(\delta_{\mathrm{C}} 137.4\right)$ and olefinic methine $\mathrm{C}-6\left(\delta_{\mathrm{C}} 124.5\right.$ $\delta_{\mathrm{H}}$ 5.22), and given the $\mathrm{H}_{3}-18$ is a singlet, it must be attached to $\mathrm{C}-7$. This was supported by HMBC correlations from $\mathrm{H}_{2}-9$ to C-7. COSY correlations from $\mathrm{H}_{3}-18$ to the olefinic methine C-6, from $\mathrm{H}-6$ to the methylene $\mathrm{CH}_{2}-5\left(\delta_{\mathrm{C}} 24.5 \delta_{\mathrm{H}} 1.95\right)$, and from $\mathrm{H}_{2}-5$ to $\mathrm{CH}_{2}-4\left(\delta_{\mathrm{C}} 36.3 \delta_{\mathrm{H}} 1.13,1.26\right)$, supported by HMBC correlations from $\mathrm{H}-5$ to $\mathrm{C}-$ 4, C-6 and C-7 allowed the addition of a further subunit to the proposed substructure (Figure 5.6).

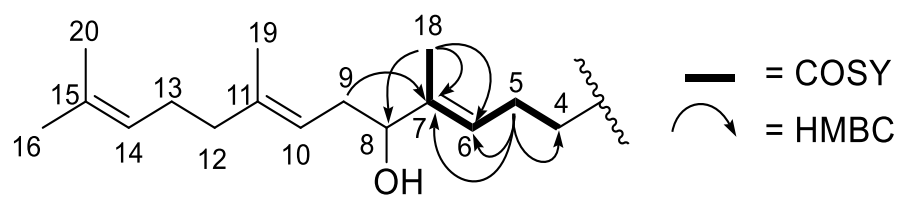

Figure 5.6 Key COSY and HMBC correlations establishing substructure 4

HMBC correlations from the methyl doublet $\mathrm{CH}_{3}-17\left(\delta_{\mathrm{C}} 19.4 \delta_{\mathrm{H}} 0.82\right)$ to the methine CH-3 $\left(\delta_{\mathrm{C}} 29.8 \delta_{\mathrm{H}} 1.82\right)$, methylenes $\mathrm{CH}_{2}-4$ and $\mathrm{CH}_{2}-2\left(\delta_{\mathrm{C}} 43.2 \delta_{\mathrm{H}} 1.81,2.02\right)$ in addition to COSY correlations from $\mathrm{H}_{3}-17$ to $\mathrm{H}_{2}-2$ and $\mathrm{H}-3$ and from $\mathrm{H}_{2}-2$ to $\mathrm{H}-3$ extended the established alkyl chain. This was further supported by HMBC correlations from $\mathrm{H}-3$ to $\mathrm{C}-4$ and $\mathrm{C}-17$ as well as from $\mathrm{H}_{2}-2$ to $\mathrm{C}-3, \mathrm{C}-4$ and $\mathrm{C}-17$ (Figure 5.7).

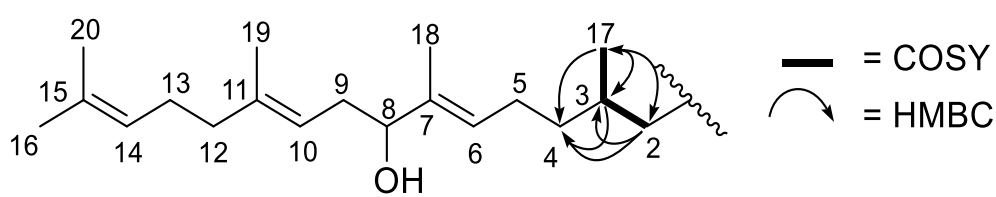

\section{Figure 5.7 Key COSY and HMBC correlations establishing substructure 5}

Further HMBC correlations from $\mathrm{H}_{2}-2$ and $\mathrm{H}-3$ to the $\mathrm{C}-1$ carbonyl $\left(\delta_{\mathrm{C}} 171.0\right)$, in addition to the HMBC correlations of the C-21 methylene ( $\delta_{\mathrm{C}} 35.5 \delta_{\mathrm{H}} 3.27$ ), to $\mathrm{C}-1$, helped assign the position of the carbonyl carbon. The chemical shifts of $\mathrm{CH}_{2}-21$ and of C-1 $\left(\delta_{\mathrm{C}} 171.0\right)$ were indicative of an amide functionality, supported by a COSY correlation from $\mathrm{H}_{2}-21$ to the exchangeable amide proton $\left(\delta_{\mathrm{H}} 7.68\right)$. HMBC correlations 
from the exchangeable amide proton to $\mathrm{C}-1$ and COSY correlation from $\mathrm{H}_{2}-21$ to $\mathrm{H}_{2}-22$ $\left(\delta_{\mathrm{C}} 50.7 \delta_{\mathrm{H}} 2.50\right)$ further extended the carbon skeleton (Figure 5.8).

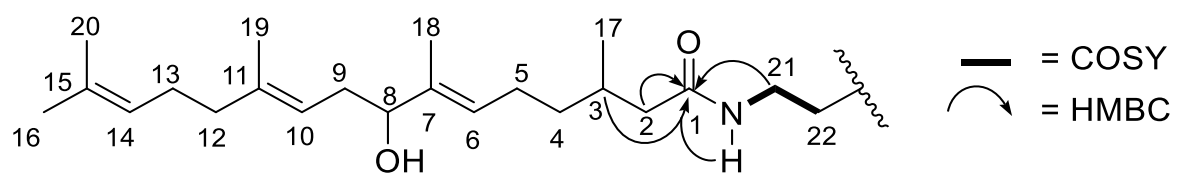

Figure 5.8 Key COSY and HMBC correlations establishing substructure 6

The substructure established to this point accounted for all the carbons required by the molecular formula, leaving $\mathrm{SO}_{3} \mathrm{H}$. As all branching termini had been accounted for, the $\mathrm{SO}_{3} \mathrm{H}$ group must be attached to $\mathrm{C}-22\left(\delta_{\mathrm{C}} 50.7\right)$, which would account for the downfield shift of this carbon resonance.

To confirm the geometry of the alkenes, a 2D ROESY experiment was performed, to determine the spatial positions of the methyls. The chemical shifts of the olefinic methyls C-18, C-19 and C-20 ( $\delta_{\mathrm{C}} 11.3,16.1$ and 17.6) were indicative of $E$ rather than $Z$ geometries, which would have approximate chemical shifts of $25.0 \mathrm{ppm}$ [259], this further confirmed the proposed $E$ geometry for the alkenes in rimarikiamide A (98) (Figure 5.9). The planar structure of $\mathbf{9 8}$ was therefore as shown in Figure 5.9.

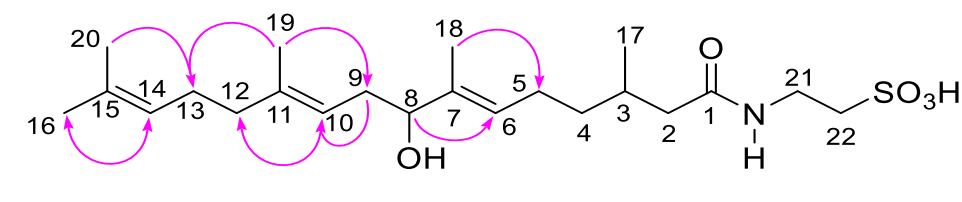

Figure 5.9 Key ROESY correlations establishing the alkene geometry of rimarikiamide A (98). 
Table $5.1{ }^{1} \mathrm{H}(600 \mathrm{MHz})$ and ${ }^{13} \mathrm{C}(150 \mathrm{MHz})$ NMR data (d6-DMSO) for rimarikiamide $\mathrm{A}(\mathbf{9 8})$.

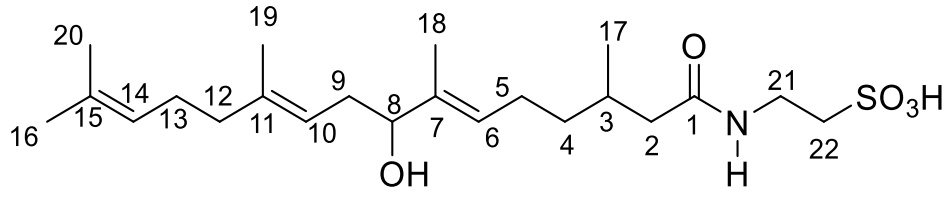

98

\begin{tabular}{|c|c|c|c|c|c|}
\hline Position & $\delta_{\mathrm{C}}$ & $\begin{array}{l}{ }^{1} J_{\mathrm{CH}} \\
(\mathrm{Hz}) \\
\end{array}$ & $\delta_{\mathrm{H}}(\mathrm{J}$ in $\mathrm{Hz})$ & COSY & HMBC \\
\hline 1 & 171.0 & & & & \\
\hline $2 \mathrm{a}$ & 43.2 & 122 & $1.81 \mathrm{~m}, 1 \mathrm{H}$ & $2 b, 17$ & $1,3,4,17$ \\
\hline $2 b$ & & 120 & $2.02 \mathrm{~m}, 1 \mathrm{H}$ & $2 \mathrm{a}, 3,17$ & $1,3,4,17$ \\
\hline 3 & 29.8 & 122 & $1.82 \mathrm{~m}, 1 \mathrm{H}$ & $2 b, 17$ & $1,4,17$ \\
\hline $4 a$ & 36.3 & 121 & $1.13 \mathrm{~m}, 1 \mathrm{H}$ & $4 b, 5$ & \\
\hline $4 \mathrm{~b}$ & & 122 & $1.26 \mathrm{~m}, 1 \mathrm{H}$ & $4 a, 5$ & \\
\hline 5 & 24.5 & 122 & $1.95 \mathrm{~m}, 2 \mathrm{H}$ & 4,6 & $4,6,7$ \\
\hline 6 & 124.5 & 152 & $5.22(\mathrm{t}, 7.0 \mathrm{~Hz}, 1 \mathrm{H})$ & 5,18 & 8,18 \\
\hline 7 & 137.4 & & & & \\
\hline 8 & 76.0 & 142 & $3.77(\mathrm{dd}, 4.1,6.5 \mathrm{~Hz}, 1 \mathrm{H})$ & 8-OH,9 & \\
\hline 9 & 33.9 & 122 & $2.08(\mathrm{t}, 6.7 \mathrm{~Hz}, 2 \mathrm{H})$ & $8,10,19$ & $7,8,10,11$ \\
\hline 10 & 121.3 & 153 & $5.04 \mathrm{~m}, 1 \mathrm{H}$ & $9,12,19$ & 12,19 \\
\hline 11 & 134.9 & & & & \\
\hline 12 & 39.3 & 122 & $1.91 \mathrm{~s}, 2 \mathrm{H}$ & 10,13 & $10,11,13,14,19$ \\
\hline 13 & 26.2 & 113 & $2.00 \mathrm{~m}, 2 \mathrm{H}$ & $12,14,16,20$ & $12,14,15$ \\
\hline 14 & 124.2 & 151 & $5.06 \mathrm{~m}, 1 \mathrm{H}$ & $13,16,20$ & \\
\hline 15 & 130.6 & & & & \\
\hline 16 & 25.6 & 125 & $1.63 \mathrm{~s}, 3 \mathrm{H}$ & $13,14,20$ & $14,15,20$ \\
\hline 17 & 19.4 & 120 & $0.82(\mathrm{~d}, 6.1 \mathrm{~Hz}, 3 \mathrm{H})$ & $2 a, b, 3$ & $2,3,4$ \\
\hline 18 & 11.3 & 123 & $1.51 \mathrm{~s}, 3 \mathrm{H}$ & 6 & $6,7,8$ \\
\hline 19 & 16.1 & 125 & $1.54 \mathrm{~s}, 3 \mathrm{H}$ & 9,10 & $10,11,12$ \\
\hline 20 & 17.6 & 126 & $1.55 \mathrm{~s}, 3 \mathrm{H}$ & 14,16 & $14,15,16$ \\
\hline 21 & 35.5 & 142 & $3.27 \mathrm{~m}, 2 \mathrm{H}$ & $1-\mathrm{NH}, 22$ & 1,22 \\
\hline 22 & 50.7 & 134 & $2.50 \mathrm{~m}, 2 \mathrm{H}$ & 21 & 21 \\
\hline $1-\mathrm{NH}$ & & & $7.68(\mathrm{t}, 5.8 \mathrm{~Hz}, 1 \mathrm{H})$ & 21 & 1 \\
\hline $8-\mathrm{OH}$ & & & $4.59(\mathrm{~d}, 4.0 \mathrm{~Hz}, 1 \mathrm{H})$ & 8 & 9 \\
\hline
\end{tabular}

Unfortunately, a lack of material at this point prevented establishing the configuration of the C-8 alcohol and C-3 methyl positions. The distance between $\mathrm{C}-8$ and $\mathrm{CH}_{3}-17$ as well as the flexible nature of the chain prevented the relative configuration from being established. Thus, the configurations of $\mathrm{C}-3$ and $\mathrm{C}-8$ remain unknown. 
One possible method to elucidate the configuration is through the use of electronic circular dichroism (ECD). When looking at the structure of 98 the two chiral centers $(\mathrm{C}$ 3 and C-8) give rise to four possible diastereomers as part of two enatiomeric pairs $(R R / S S$ and $R S / S R)$. The ECD curves could be predicted for each of the four possible configurations and compared to the actual curve of $\mathbf{9 8}$. This would allow us to deduce the absolute configuration of the natural product [260]. However as there is no CD instrument available at VUW this approach was not possible. It should also be noted that calculating ECD spectra can lead to erroneous data when dealing with flexible molecules, where different conformers can give enatiomeric spectra [261]. Therefore the best way to elucidate the absolute configuration would be through the synthetic route.

\subsubsection{Biogenesis}

As rimarikiamide A is a novel taurinated diterpene, a biogenesis of (98) is proposed below. The two main building blocks to the terpenes are dimethylallyl pyrophosphate (DMAPP) and isopentenyl pyrophosphate (IPP), which could be products of the mevalonate pathway or the mevalonate-independent pathway [262]. DMAPP and IPP are condensed together by geranyl diphosphate synthase to give geranyl diphosphate (GPP) [262]. GPP and IPP are then condensed by farnesyl diphosphate synthase to generate farnesyl diphosphate (FPP), which is further condensed with IPP to give geranylgeranyl diphosphate (GGPP) [262]. GGPP is proposed to be hydrolysed to generate the alcohol geranylgeraniol, which is oxidised to produce the carboxylic acid. At this point a thioesterase could act upon the carboxyl to generate a thioester. The next step could involve the reduction of the double bond closest to the carbonyl via a reductase. Following this the oxidation of the C-8 carbon after the second isoprene unit could occur, introducing the hydroxyl functionality. The last two aforementioned steps could also occur in the opposite order, without a directed study there is no clear way of stating which step comes first. A final taurination step where taurine replaces the thioester linked enzyme is proposed to generate 98 (Figure 5.10). Compared to all the described taurinated terpenoids in section 5.1.1.5, 98 is a simple linear diterpene, without any cyclisation or any aromatic constituents. The simplicity of the molecule would mean a synthetic route should be fairly plausible, which would allow for the absolute configuration to be resolved. 


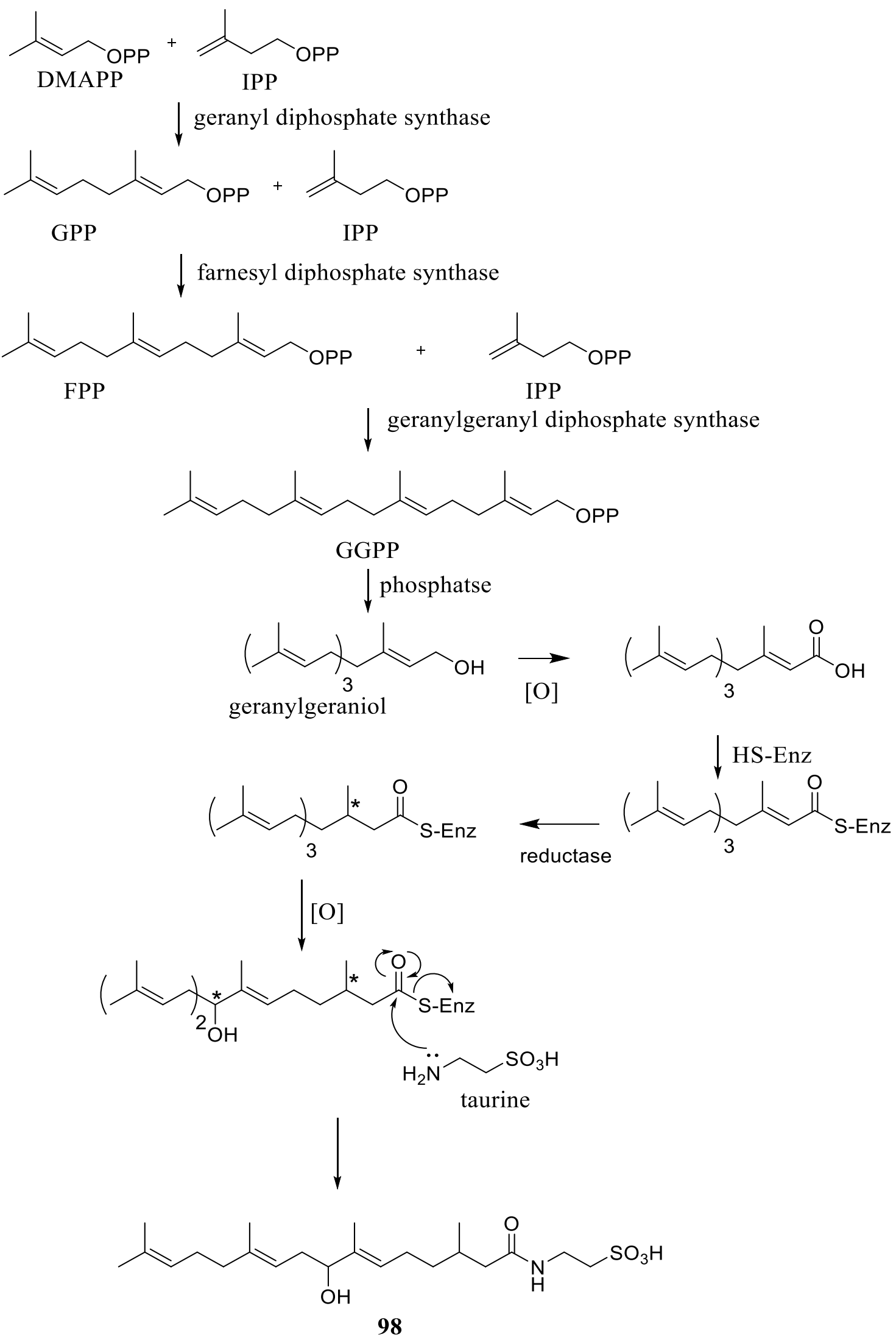

Figure 5.10 Proposed biogenesis of rimarikiamide A (98). 


\subsubsection{Reported compounds from Latrunculia sp.}

To date, a range of compounds have been isolated from Latrunculia sponges of these, four classes emerge as the most common, these are the latrunculins, trunculins, discorhadbins and callipeltins.

The latrunculins are a class of actin depolymerizing agents [263]. Latrunculin A (99) and B (100), isolated from Latrunculia magnifica, later re-classified as Negombata magnifica [264], have displayed cytotoxic effects on cell lines at sub-micromolar concentrations [265]. Studies indicate 99 binds to G-actin monomers [266], and both 99 and $\mathbf{1 0 0}$ have been shown to modulate actin stress filaments, altering the cytoskeleton and affecting cell morphology [267].<smiles></smiles>

99

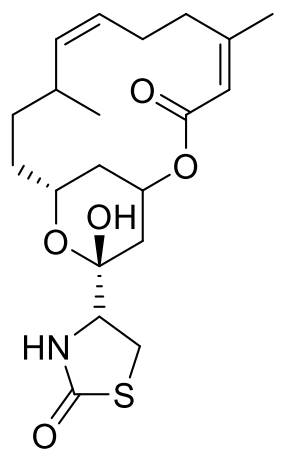

100

Trunculins A (101) and B (102) were isolated from an Australian Latrunculia sp. later re-classified as either Dicarnus or Sigmaceptrella sp. [264], and were active against Bacillus subtilis as well as Saccharomyces cerevisiae [268].

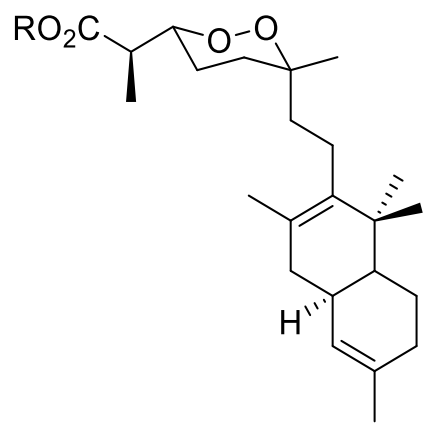

$101 \mathrm{R}=\mathrm{H}$

$102 \mathrm{R}=\mathrm{CH}_{3}$ 
The discorhabdins belong to a class of compounds termed pyrroloiminoquinones, these molecules have displayed various bioactivities such as antibacterial, anti-viral, antimalarial, cytotoxic and anti-tumour activity [269]. The highly potent low micro- to nano-molar level of activities of these compounds made them of high interest, however, the lack of selectivity of these compounds prevented them progressing as anti-cancer agents [269]. These compounds have also displayed activity against $M$. tuberculosis in addition to a range of Gram-positive and Gram-negative bacteria [269]. Below is the structure of discorhabdin $\mathrm{C} \mathbf{( 1 0 3 )}$ the first reported discorhabdin, isolated from a New Zealand Latrunculia sp. [270]. For further information on this class of compounds, please refer to two extensive reviews by $\mathrm{Hu}$ et al. and Antunes et al. [264, 269].

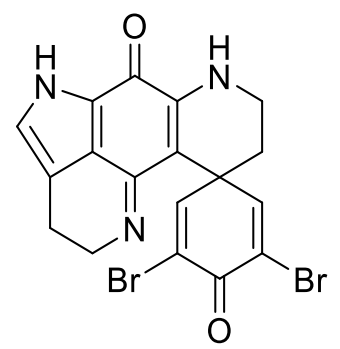

103

Callipeltins F-I are peptides isolated from Latrunculia sp. from Vanuatu which have been have been reported to have anti-Candida activity at $1 \mathrm{mM}$ [271]. Below is the structure of Callipeltin F (104).

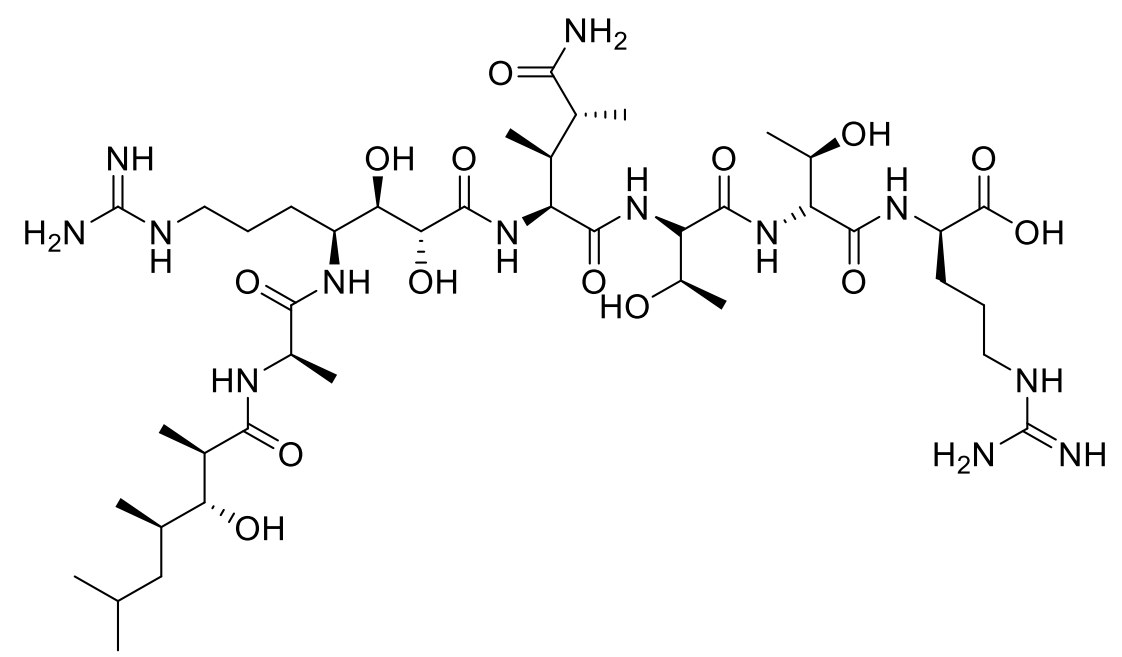


As these four classes of molecule represent the four most common classes of compounds isolated from Latrunculia sp., it is possible that very low levels of any of these compounds could be responsible for the anti-tubercular activity. To identify if any of these compounds were present in the bioactive fractions, ${ }^{1} \mathrm{H}$ NMR signals of ND4_47D+E were compared to identify signature resonances to indicate the presence of any of these compounds. However, none of the diagnostic signals for any of these compounds were identifiable in the ${ }^{1} \mathrm{H}$ NMR spectra of the active fraction (Figure 5.11).

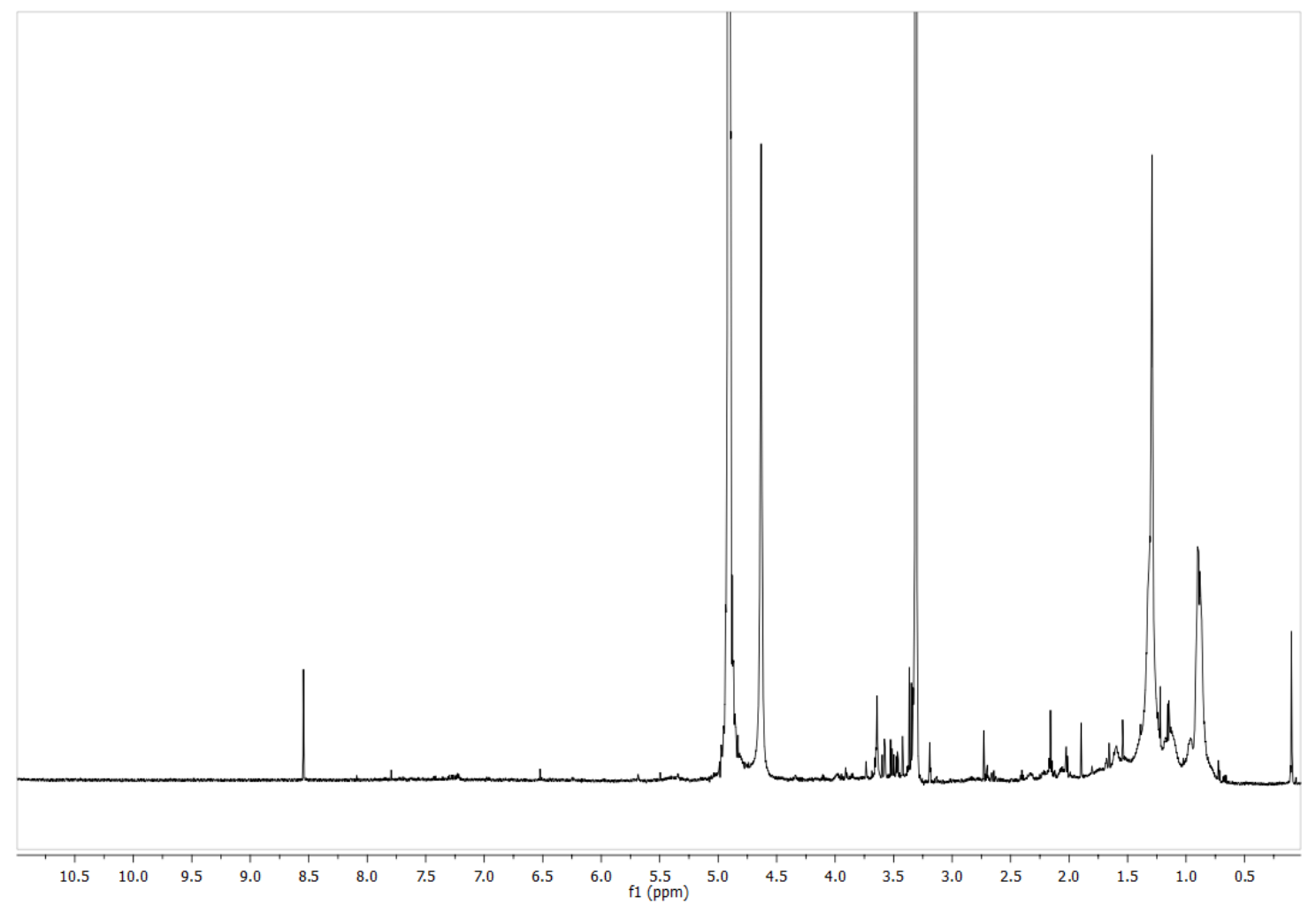

Figure 5.11 ${ }^{1} \mathrm{H}$ NMR $\left(\mathrm{CD}_{3} \mathrm{OD}, 600 \mathrm{MHz}\right)$ spectrum spectra of ND4_47D+E.

\subsubsection{Bioactivity}

With only minimal amounts of $\mathbf{9 8}$ remaining, and with no anti-mycobacterial activity linked to this compound, it was decided to investigate the effects of this compound in mammalian cell line assays. Comparison of $\mathrm{IC}_{50}$ data from the HL-60 and HEK cell line assays indicated an interesting result. HL-60 cells were chosen to represent cancer cells, while HEK cells were chosen to exhibit the general mammalian cytotoxic effects of the compound. The effectiveness of compounds can be judged by their therapeutic index 
(TI), which is the general cytotoxicity divided by the desired activity. Therefore, the $\mathrm{IC}_{50}$ value in HEK cells $(61.1 \mu \mathrm{M})$ divided by the $\mathrm{IC}_{50}$ in HL-60 cells $(9.8 \mu \mathrm{M})$ would identify a rough therapeutic index of 6 for rimarikiamide A (Figure 5.2). This is indicative of specificity for in vitro anti-cancer activity compared to generic toxicity. TI is an indicator of the potential of drug candidates [272]. High TI's are generally ideal at the early stages of drug development to avoid progression of candidates that may fall out further downstream of the development process [272]. As a compound may have an ideal TI value in vitro, in vivo higher levels of the compound may be required, which could lead to undesired side effects, thus over interpretation of the TI value should be cautioned [272]. Compounds can be modified to allow for improvements in the TI. However the lack of sufficient compound meant that any further work such as mechanism of action studies could not be performed.

\subsubsection{Future work}

To carry out further studies of the bioactivity of rimarikiamide A, more compound will be required, and identifying an efficient route for synthesis will aid in this avenue of research. However, before any future work can be done, the relative and absolute configuration of the molecule must be elucidated. Pursuing the synthetic route to generate two of the possible four stereoisomers and comparing optical rotation data and NMR spectra of the synthesized compound to that of the natural product will confirm the planar structure and will establish the configuration of the natural product. These studies are currently underway in our laboratory. 


\section{Chapter 6 3-Epi-xestoaminol C}

from Xiphophora chondrophylla. 


\subsection{Background}

Screening the VUW_MNP extract library also identified a brown alga Xiphophora chondrophylla as a hit, which displayed inhibitory activity against all three species of bacteria that were screened (Figure 3.3). Since the crude extract displayed similar levels of activity against $M$. tuberculosis $\mathrm{H} 37 \mathrm{Ra}\left(\mathrm{MIC}_{90} \geq 200 \mu \mathrm{g} / \mathrm{mL}\right)$ as M. smegmatis (MIC $_{90} \geq 200 \mu \mathrm{g} / \mathrm{mL}$ ) (Table 3.3), the fractionation process was guided by assays against $M$. smegmatis. As stated earlier, the strain of $M$. smegmatis used allows for faster assay times and the kanamycin resistance marker allows for antibiotic use to overcome contamination issues. Once the bioactive compound was isolated, the goal would be to validate it against M. tuberculosis H37Ra and, should it prove potent enough, its activity against clinical isolates would also be analysed. The compound's cytotoxic activity against mammalian cell lines would also be studied to evaluate its potential as an anti-tubercular drug. This work led to the isolation of a new 1-deoxysphingoid, a class of molecules also known as 2, 3 amino alcohols. This chapter discusses the isolation and structure elucidation of the new amino alcohol as well as early mechanism of action studies. In addition, an overview of marine derived amino alcohols as well as known biological activities linked to these compounds is also described.

\subsubsection{Marine derived amino alcohols}

Amino alcohols or 1-deoxyshpingoids are a class of compounds that contain hydroxyl and amino functional groups on hydrocarbon chains of a lipid. Their isolation has predominantly been associated with tunicates [273-282]. The range of activities these compounds have displayed include anti-cancer, antimicrobial and anti-fungal activities [273-282]. The first reported isolation of an aliphatic amino alcohol was from a Xestospongia sp. sponge [283] followed by the isolation of xestoaminols from another Xestospongia [284]. Of all the reports to date, the isolation of aliphatic amino alcohols from marine algae is unprecedented. 


\subsubsection{Alkyl chains with varying levels of unsaturation}

This section describes amino alcohols with varying alkyl chain lengths and unsaturation levels, which represent the most common class of amino alcohols. The first reported isolation of aliphatic amino alcohols was in 1988, with 2-(S)-aminotetradecan-5,7-dien3-(S)-ol (105) and 2-(S)-aminotetradecan-5,7-dien-3-(R)-ol (106) from a Papua-New Guinea Xestospongia sp. sponge. These compounds possessed inhibitory activity against Candida albicans at $19 \mu \mathrm{g} / \mathrm{mL}$ in a disk diffusion assay [283]. Similarly another group of aliphatic amino alcohols, xestoaminols A-C (107-109) were isolated from a Fijian Xestospongia sp.. Compound $\mathbf{1 0 7}$ displayed activity against the bacteria S. aureus, $S$. pyogens and E. coli, and the fungi C. albicans and Trichphyton mentagrophytes in disk diffusion assays at $100 \mu \mathrm{g} / \mathrm{mL}$. In addition, both 107 and 109 displayed extremely weak inhibitory activity in a reverse transcriptase assay at $1 \mathrm{mg} / \mathrm{mL}$ [284].

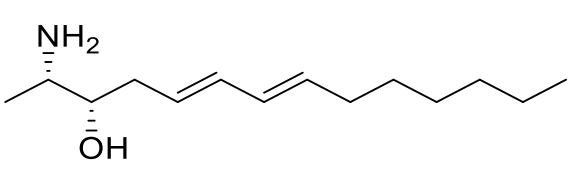

105<smiles>CCCCCC/C=C/C=C/C[C@H](O)[C@H](C)N</smiles>

106<smiles>[R]CCCCCCC(O)C(C)N</smiles>

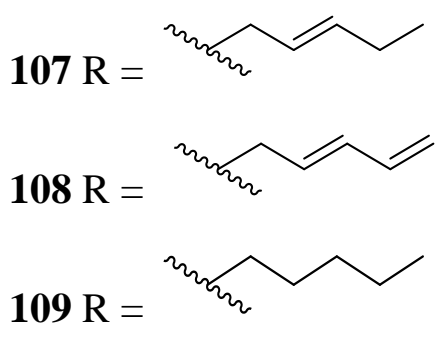

(2S, 3R)-2-Aminododecan-3-ol (110) was reported from the ascidian Clavelina oblonga and displayed anti-fungal activity against $C$. albicans with an MIC of $0.7 \mu \mathrm{g} / \mathrm{mL}$ [273]. Crucigasterins 277, 275 and 225 (111-113) were first isolated in 1993 from a Mediterranean tunicate Pseudodistoma crucigater with moderate activity reported against L1210, B. subtilis and S. cerevisiae [277]. 
<smiles>CCCCCCCCC[C@@H](O)C(C)N</smiles>

110<smiles>C=C(C)/C=C/CC(C)(C)C/C=C/C[C@H](O)C(C)N</smiles>

112<smiles>CC(N)[C@@H](O)C/C=C/CC(C)(C)/C=C/C(C)(C)C</smiles>

111<smiles>C=CCCCCCCC/C=C/[C@@H](O)[C@H](C)N</smiles>

113

Crucigasterins A-E (114-118) were also isolated from $P$. crucigater, with 115 displaying the best activity of $50 \mu \mathrm{g} / \mathrm{mL}$ against E. coli and C. albicans, while 118 was active at $100 \mu \mathrm{g} / \mathrm{mL}$ against $E$. coli [276].<smiles>C=CCCCC/C=C/C=C/C[C@H](O)C(C)N</smiles>

114<smiles>C=CCCC=CC[C@@H](O)C(C)N</smiles>

115<smiles>C=CC=CCCC(C)C(C)N</smiles>

116<smiles>CCCCCC/C=C/CC/C=C/C[C@H](O)C(C)N</smiles>

117<smiles>[Z]C(C)(C=CC(C)(C)CC=CC[C@@H](O)C(C)N)CCCC</smiles>

118

Closely related to the crucigasterins are the haloaminols A-C (119-121), isolated from a Haliclona sponge from the Great Barrier Reef, which have been reported to inhibit the growth of E. coli, B. subtilis, C. albicans, T. mentagrophytes and C. resinae [285]. The researchers also identified haloaminol D (122) isolated as the $N$-acetyl derivative. 
<smiles>C=CCCCC(O)C(C)N</smiles>

119<smiles>CCCCC=CCC(O)C(C)N</smiles>

121<smiles>C=CCCCC(O)C(C)N</smiles>

120<smiles>CC(/C=C/C(C)(C)C)CC(O)C(C)N</smiles>

122

Obscuraminols A-F (123-128), also closely related to the crucigasterins and haloaminols, were isolated from the ascidian Pseudodistoma obscurum. These compounds were reported to be active against P388, A-549 and HT-29 cell lines with $\mathrm{IC}_{50}$ values of $0.5,0.2$ and $0.5 \mu \mathrm{g} / \mathrm{mL}$ respectively [278]. Haloaminol $\mathrm{D}$ and Obscuraminol A are in fact the same compound, however $\mathbf{1 2 2}$ was initially isolated as the $\mathrm{N}$-acetyl derivative only four months prior to the isolation of $\mathbf{1 2 3}$.

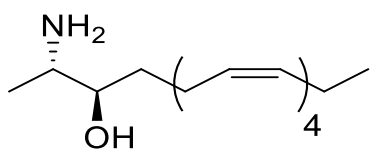

123<smiles>C=CCCC=CCCCC(C)[C@H](N)O</smiles>

125<smiles>CCCCC=CCCCC(O)C(C)N</smiles><smiles>C=CCCCCCC/C=C/CCCC(O)C(C)N</smiles>

124<smiles>CCCCCC/C=C/CCCCCC(O)C(C)N</smiles>

126<smiles>C=CCCCC(O)C(C)N</smiles> 
Clavaminols A-F (129-134) were isolated from the ascidian Clavelina phlegrae. The most potent of these aliphatic amino alcohols, 129, had an $\mathrm{IC}_{50}$ value of $5 \mu \mathrm{g} / \mathrm{mL}$ against a gastric tumour cell line (AGC cells), lung cancer cell line (A-549) and a breast cancer cell line (T47D). In addition, 129 was shown to inhibit cell growth by inducting apoptosis in the G1-S phase of the cell cycle in Jurkat cells [275].
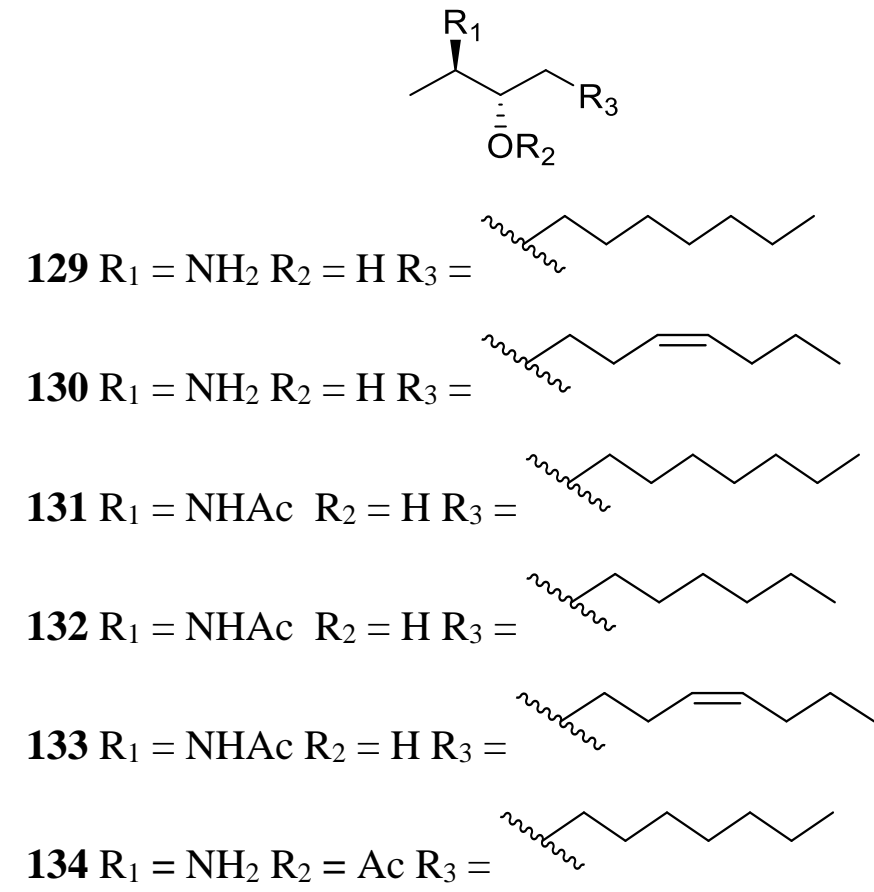

The final example in this class of amino alcohols, spisulosine or ES-285 (135), has drawn much interest. Initially isolated from the marine mollusc Spisula polynyma, this compound has drawn major interest due to its activity against solid tumours such as colorectal, renal and prostate tumors, amongst others, for which it progressed to phase 1 clinical trials [286-289].

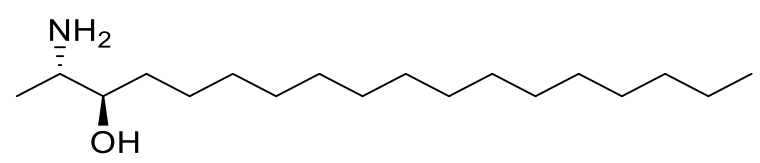

135

The first trial indicated doses ranging from $4-128 \mathrm{mg} / \mathrm{m}^{2}$ were manageable and showed no dose limiting toxicities. However, at $256 \mathrm{mg} / \mathrm{m}^{2}$ one of the four patients receiving treatment displayed neurotoxicity, a side effect responsible for the fatality of one of the 
three patients treated at $200 \mathrm{mg} / \mathrm{m}^{2}$. Overall this study reported that at $128 \mathrm{mg} / \mathrm{m}^{2}$ patients achieved desired pharmacokinetic and pharmacogenetic changes [289].

In all the clinical trials, only moderate anti-tumor activity was observed, in addition to side effects which included elevated hepatic and neural toxicities, anaemia, lymphopenia, asthenia and nausea. This led to the discontinuation of the clinical development of this compound [286-288].

The mode of action for $\mathbf{1 3 5}$ has not been fully elucidated, although the research to date proposes that the compound may work through multiple pathways. The induction of cell death in vitro has been shown to occur as a result of disruption of actin cell fibres; this destabilization of the cytoskeleton has been shown to not affect the microtubules of cells [290]. The activation of caspase 3 and 12, the phosphorylation of p53 and the activation of isoforms of protein kinase $\mathrm{C}(\mathrm{PKC})$ have all been shown to induce atypical cell death [291]. Further work in a prostate cancer cell line PC-3 focusing on the effects of spisulosine on PKC indicated the activation of the isoform PKC 5 [292], increasing ceramide levels, which has been linked to induction of apoptosis [293-295]. The most recent research has focused on how ES-285 affects ceramide levels in MDA MB 468 breast cancer cells. Treating cells with the compound, as well as analogues, caused an increase in $\mathrm{N}$-C18 1-deoxyceramide levels, indicating ES-285 as the preferred substrate for acylation [296], a quality that identifies this compound as a probe for ceramide synthase activity.

\subsubsection{Cyclised amino alcohols}

This section describes the limited number of amino alcohols that contain cyclic structures. The pseudodistomins A (136) and B (137), isolated from the tunicate Pseudodistoma kanako in 1986, represent the first amino alcohols to be reported. These cyclic piperidine alcohols inhibited the growth of mouse leukaemia cell lines L1210 with $\mathrm{IC}_{50}$ values of 2.5 and $0.4 \mu \mathrm{g} / \mathrm{mL}$, and L5178Y with values of 2.4 and $0.7 \mu \mathrm{g} / \mathrm{mL}$, respectively [280, 297]. Pseudodistomin C (138) was later isolated from P. kanoko, with reported activity against the L1210 cell line and a human epidermoid carcinoma (KB cells) with $\mathrm{IC}_{50}$ values of 2.6 and $2.3 \mu \mathrm{g} / \mathrm{mL}$, respectively. Shortly after, pseudodistomins D-F (139-141) were reported from the ascidian P. malgrava [281]. In addition to the re-isolation of $\mathbf{1 3 7}$ and $\mathbf{1 3 8}$, the authors reported the activity of 137-141 
against $S$. cerevisiae at a range of concentrations between $110-200 \mu \mathrm{g} / \mathrm{mL}$ in a zone of inhibition assay [281].

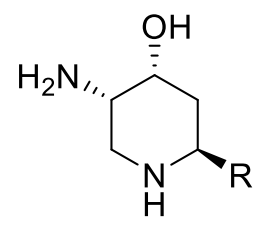

$136 \mathrm{R}=\mathrm{A}$ $137 \mathrm{R}=\mathrm{B}$ $141 \mathrm{R}=\mathrm{C}$<smiles>[R]C1C[C@@H](O)[C@@H](N)CN1</smiles>

$138 \mathrm{R}=\mathrm{C}$ $140 \mathrm{R}=\mathrm{B}$<smiles>[R]C1C[C@@H](O)[C@@H](N)CN1</smiles>

$139 \mathrm{R}=\mathrm{B}$

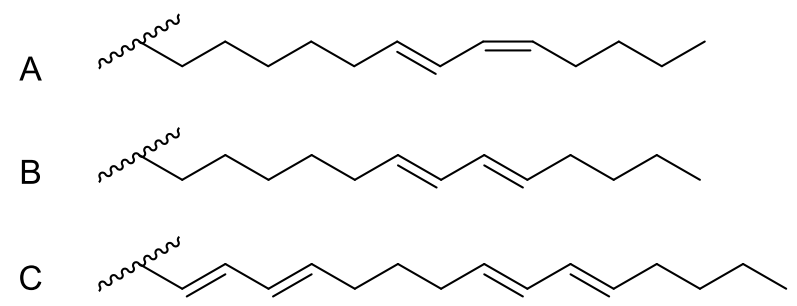

Containing a fused bicyclic ring as part of the linear chain, amaminols A (142) and B (143) were reported from a tunicate belonging to the family Polyclinidae, with $\mathrm{IC}_{50}$ values of $2.1 \mu \mathrm{g} / \mathrm{mL}$ against P388 murine leukaemia cells [274].

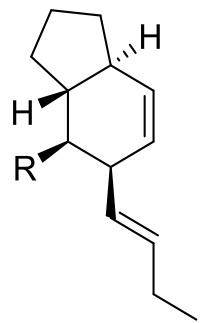<smiles>[R12]#CC(C)N</smiles><smiles></smiles>

\subsubsection{3 $\alpha, \omega$-Double headed amino alcohols}

This section covers compounds with amino alcohol polar head groups at both ends of the chain. Leucettamols A (144) and B (145) were isolated from the sponge Leucetta microraphis, with moderate activity reported against B. subtilis [298]. Leucettamol A was recently reported to inhibit the interaction of Ubc13 with Uev1A, 
with an $\mathrm{IC}_{50}$ of $50 \mu \mathrm{g} / \mathrm{mL}$. This inhibition is believed to increase p53 levels and therefore makes an ideal target for anti-cancer agents, [299].<smiles>CC(N)[C@H](O)CCCCCC(C)(C)/C=C/C(C)(C)[C@@H](O)C(C)N</smiles>

\section{4}<smiles>CC(C=CC(O)CC=CC[C@@H](O)C(C)N)=CC(C)(C)CCCCC[C@H](O)[C@H](C)N</smiles>

145

BRS1 (146), isolated from an Australian calcareous sponge, is very closely related to the leucettamols, with reported inhibition of PKC activity with an $\mathrm{EC}_{50}$ of $98 \mu \mathrm{M}$ [300]. The final example for compounds belonging to this group is rhizocalinin B (147), isolated from a North-Western Australian sponge Oceanapia sp. with no reported bioactivity [301].<smiles>CC(N)C(C)C=CCC(C)(C)C=CC(C)(C)CC=CC=CC(O)C(C)N</smiles>

146<smiles>CCCOC(CC(=O)CCCCCCCCCCCCC(C)N)C(C)N</smiles>

\section{7}

In addition to having the bis-amino alcohol head groups, the compounds listed below also contain carbohydrates as part of their structure. Rhizochalin (148) was isolated from a Madagascan sponge Rhizochalina incrustata with reported inhibitory activity against $S$. aureus [302]. Rhizochalinin A (149), also isolated from $R$. incrustata, is a sphingolipid aglycone that has anti-leukemic activity against human monocytic THP-1 cells, with a reported $\mathrm{IC}_{50}$ of $7.5 \mu \mathrm{M}$ [303]. Rhizochalin B (150) was isolated from a 
North-Western Australian sponge Oceanapia sp. [301], while rhizochalins C (151) and D (152) were isolated from $R$. incrustata [304]. There has been no reported activity for rhizochalins B-D.

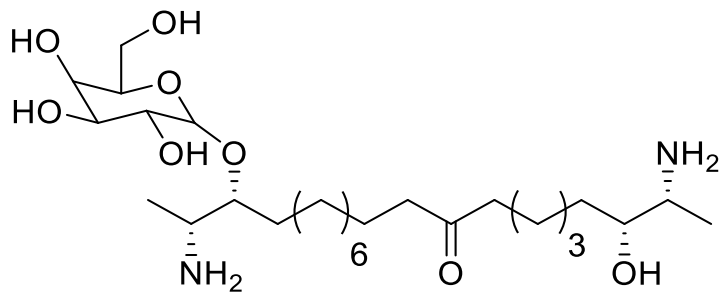

148

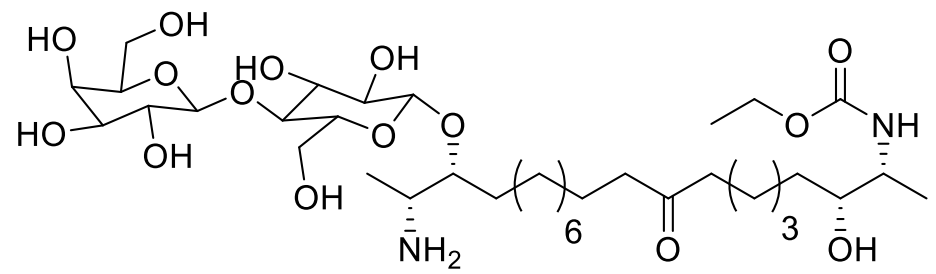

\section{9}
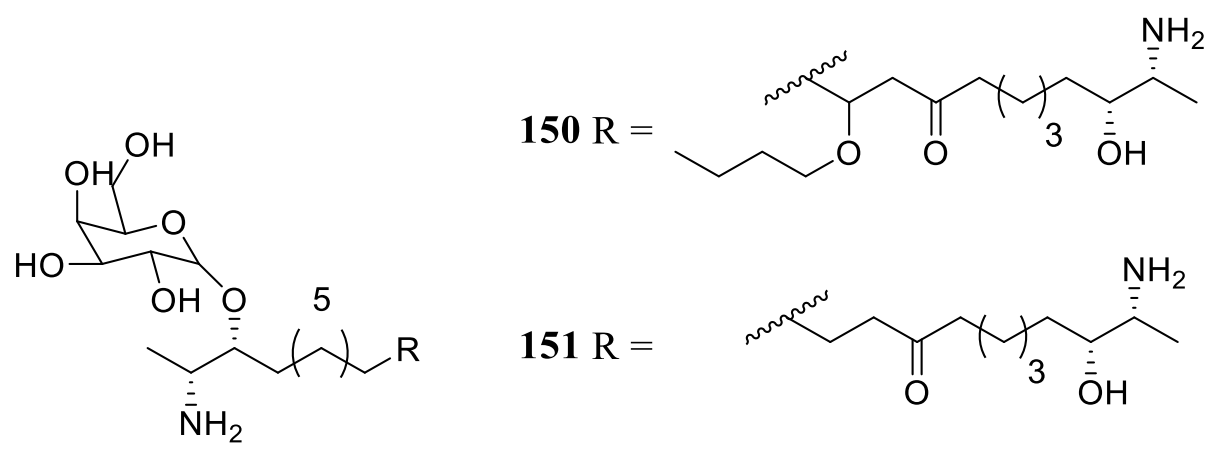

$152 \mathrm{R}=$<smiles>CCCCC(=O)CCC(C)(C)CC[C@@H](O)[C@@H](C)N</smiles>

Isorhizochalin (153) is an epimer of rhizochalin also isolated from $R$. incrustata, although no bioactivity has been linked to this compound [305]. 


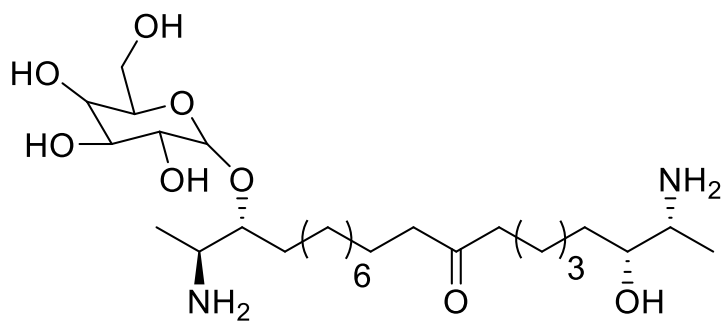

153

Oceanapiside (154), another sphingolipid, was isolated from the South Australian sponge Oceanapia phillipensis. This compound varies from the rhizochalins near the terminus that contains the glucose, with true sphingosine head-group unlike that found in the rhizochalins. Oceanapiside has a reported MIC of $10 \mu \mathrm{g} / \mathrm{mL}$ against Candida glabrata [306].<smiles>CC(N)C(O)C(=O)NCCCC(=O)CCCC1OC2OC(CO)C(O)C(O)C(O)C1OC2N</smiles>

\section{4}

Calyxoside (155) isolated from a Calyx sponge is a sphingolipid that was reported to have inhibitory activity in rad 52 and 51 knock outs of S. cerevisiae strains at 62 and $36 \mu \mathrm{g} / \mathrm{mL}$, respectively, in a zone of inhibition assay. In addition, it has reported activities against the HFF, MRC-5, SW480, HT-29, Saos-2, DLD-1 and H460 cell lines with $\mathrm{IC}_{50}$ values between $3-20 \mu \mathrm{g} / \mathrm{mL}$ [307]. It also contains a formal sphingosine head.

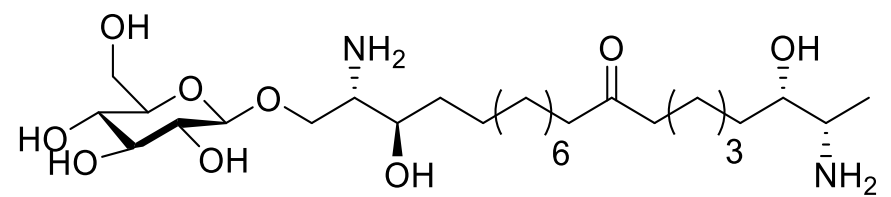




\subsection{Methods}

\subsubsection{Extraction of algae}

Xiphophora chondrophylla (300 g) was extracted twice with methanol (MeOH, $400 \mathrm{~mL})$ that was cyclic loaded onto a $120 \mathrm{~mL}$ HP20 column, following which the column was eluted with $360 \mathrm{~mL}$ mixtures of $20 \%, 40 \%, 60 \%, 80 \%$ and $100 \%$ acetone in water. Except for the $100 \%$ acetone, all other fractions were back loaded onto $60 \mathrm{~mL}$ columns and eluted with $180 \mathrm{~mL}$ of $\mathrm{MeOH}$ followed by $180 \mathrm{~mL}$ of acetone, fractions were dried down in vacuo and transferred to pre-weighed vials. Based on bioactivity, the $40 \%$ acetone fraction was further pursued, half of the mass $(350 \mathrm{mg}$ ) was fractionated using a $40 \mathrm{~mL}$ HP20ss column, eluting with $120 \mathrm{~mL}$ of $30 \%, 40 \%, 50 \%, 60 \%, 80 \%$ and $100 \%$ mixtures of $\mathrm{MeOH}$ in $\mathrm{H}_{2} \mathrm{O}$; the $30 \%-60 \%$ fractions were collected in test tubes. Guided by bioassays, test tubes $43-46(50 \% \mathrm{MeOH}$ fractions, $17.8 \mathrm{mg})$ were fractionated a further three times using $\mathrm{LH} 20$ size exclusion chromatography using $90 \% \mathrm{MeOH} / \mathrm{H}_{2} \mathrm{O}$. The final fractionation was performed using a $1 \mathrm{~mL}$ DIOL column and eluted with $3 \mathrm{~mL}$ volumes of $0 \%, 25 \%, 50 \%, 75 \%$ and $100 \%$ mixtures of ethyl acetate (EtOAc) in hexanes followed by $25 \%, 50 \%, 75 \%$ and $100 \% \mathrm{MeOH}$ in EtOAc, finally the column was stripped with $50 \%$ and $75 \% \mathrm{H}_{2} \mathrm{O}$ in $\mathrm{MeOH}$ to give 3-epi-xestoaminol C (156) (0.9 $\mathrm{mg})$.

Based on the results of the initial isolation, an optimised method was used on the second half of the $40 \%$ acetone fraction. The remaining 40\% HP20 fraction (350 mg) was separated on a $30 \mathrm{~mL}$ DIOL column, eluting with $90 \mathrm{~mL}$ volumes of $0 \%, 25 \%, 50 \%, 75 \%$ and $100 \%$ mixtures of EtOAc in hexanes followed by $25 \%, 50 \%, 75 \%$ and $100 \%$ $\mathrm{MeOH}$ in EtOAc, finally the column was stripped with $50 \% \mathrm{H}_{2} \mathrm{O}$ in $\mathrm{MeOH}$. The $0 \%, 25 \%$ and $50 \%$ EtOAc in hexanes and 25\%, 50\%, 75\% and $\mathrm{MeOH}$ in EtOAc elutions were collected in test tubes. Test tubes 47-50 (25\% MeOH/ EtOAc) were pooled together (248.1 mg) and separated further on a $25 \mathrm{~mL}$ DIOL, eluting with $75 \mathrm{~mL}$ volumes of the same solvent mixtures for the earlier column. ${ }^{1} \mathrm{H}$ NMR identified test tubes 6-13 to contain 3-epi-xestoaminol $\mathrm{C}$, the combined mass (185 $\mathrm{mg})$ was dissolved in dichloromethane and partitioned against $1 \mathrm{M} \mathrm{HCl}$, the organic layer was collected and dried, the acidic aqueous layer was then neutralised with $1 \mathrm{M} \mathrm{NaOH}$ and this was partitioned against dichloromethane again. Following the $\mathrm{HCl}$ and $\mathrm{NaOH}$ washes, a 
total of $117 \mathrm{mg}$ was isolated, which was divided into thirds and fractionated on three DIOL columns using the same eluting conditions for all columns, $0 \%, 50 \%$ and $100 \%$ mixtures of EtOAc in hexanes (collected in bulk) followed by $25 \%, 50 \%$ and $100 \%$ $\mathrm{MeOH}$ in EtOAc (collected in test tubes), finally the column was stripped with $50 \%$ $\mathrm{H}_{2} \mathrm{O}$ in $\mathrm{MeOH}$. This afforded a total of $58 \mathrm{mg}$ of 3-epi-xestoaminol C (156) (test tubes 5-7).

3-Epi-xestoaminol C: $[\alpha]^{25} \mathrm{D}-6.19\left(c\right.$ 0.42, $\left.\mathrm{CHCl}_{3}\right)$; IR $v_{\max } 3367,2954,2871,1602 \mathrm{~cm}^{-1}$; $\mathrm{CD}_{3} \mathrm{OD}$ NMR data see Table 6.2; ${ }^{1} \mathrm{H}$ NMR $\left(\mathrm{CDCl}_{3}, 600 \mathrm{MHz}\right) \delta 3.65(1 \mathrm{H}, \mathrm{t}, J=8.5 \mathrm{~Hz}$, H-3), 3.32 (1H, m, H-2), 1.56 (1H, m, H-4b), 1.53 (1H, m, H5-b), 1.40 (1H, m, H-4a), $1.38(3 \mathrm{H}, \mathrm{d}, J=6.6 \mathrm{~Hz}, \mathrm{H}-1), 1.35$ (1H, m, H-5a), 1.27 (2H, m, H-13), 1.25 (14H, m, H6 to $\mathrm{H}-12), 0.88(3 \mathrm{H}, \mathrm{t}, J=7.0 \mathrm{~Hz}, \mathrm{H}-14) ;{ }^{13} \mathrm{C} \mathrm{NMR}\left(\mathrm{CDCl}_{3}, 150 \mathrm{MHz}\right) \delta 73.0(\mathrm{C}-3)$, 53.3 (C-2), 33.6 (C-4), 32.1 (C-12), 29.88-29.52 (C-6 to C-11), 25.5 (C-5), 22.9 (C-13), 15.8 (C-1), 14.3 (C-14) ; HRESIMS m/z $230.2467[\mathrm{M}+\mathrm{H}]^{+}$(calcd for $\mathrm{C}_{14} \mathrm{H}_{32} \mathrm{NO}$, 230.2478).

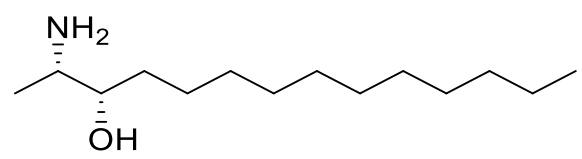

156 


\subsubsection{Isolation schemes for 3-epi-xestoaminol C (156)}

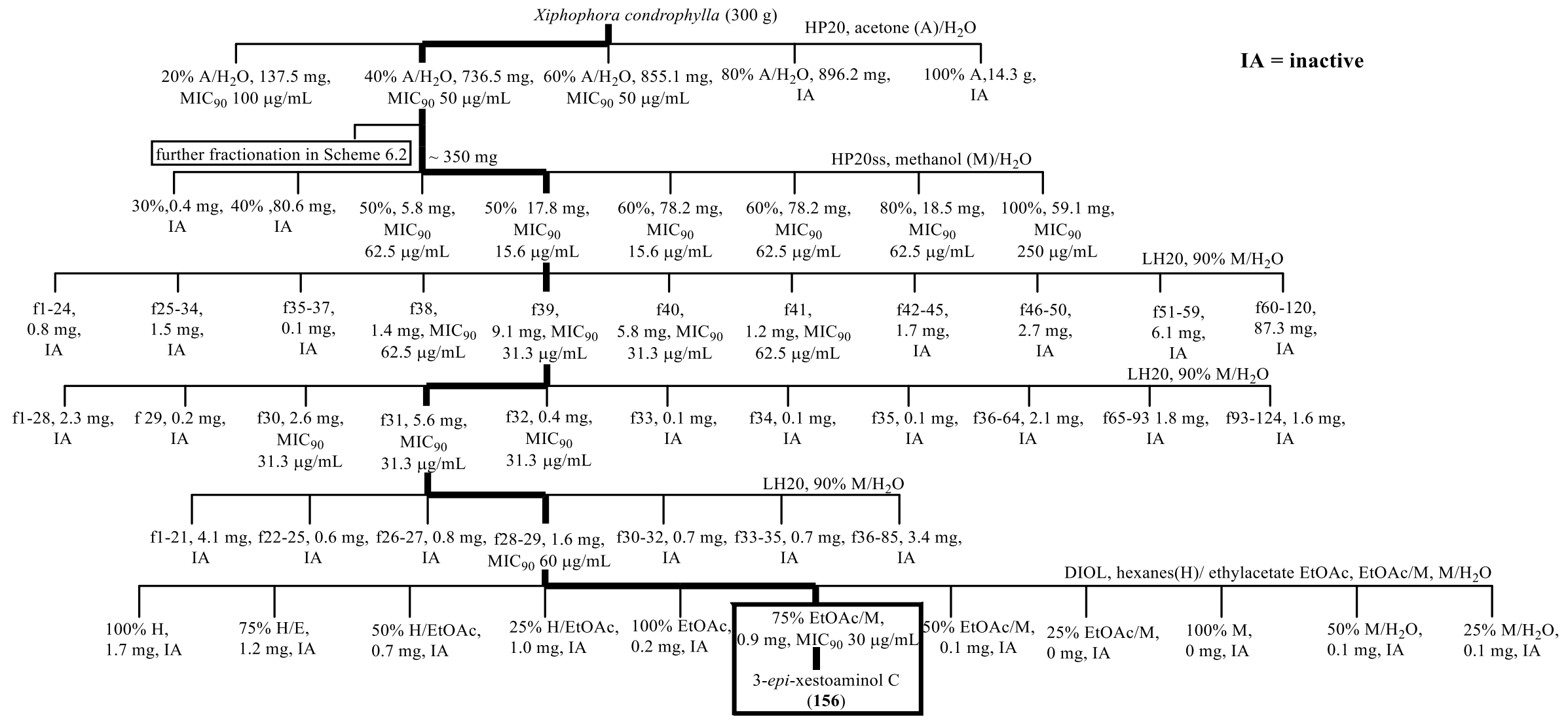

Scheme 6.1 Original isolation scheme for 3-epi-xestoaminol C (156) from Xiphophora chondrophylla. 


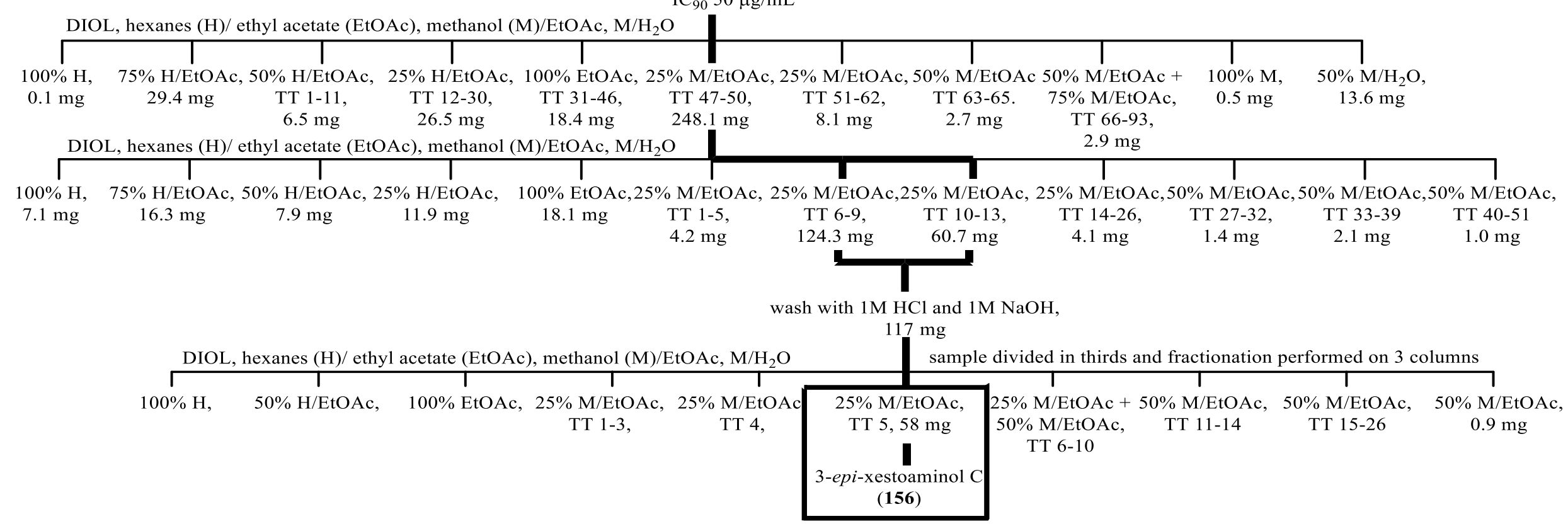

\section{Scheme 6.2 Optimised isolation scheme for 3-epi-xestoaminol C (156) from Xiphophora chondrophylla.}




\subsubsection{Derivatisation reactions}

\subsubsection{Oxazolidinone derivative}

3-Epi-xestoaminol C (156) (3 mg) was stirred with 1,1' carbonyldiimidazole (CDI, 3 mg, 1.4 equiv.) for $24 \mathrm{~h}$ in DCM. Monitoring via TLC (running conditions 1:1 $\mathrm{MeOH}: \mathrm{EtOAc}$ ) showed the reaction had not gone to completion, therefore further CDI (7 mg, 3.3 equiv.) was added to the reaction, after $2 \mathrm{~h}$ the reaction had gone to completion. The reaction mixture was partitioned against $\mathrm{H}_{2} \mathrm{O}$ three times and the organic layer was dried, yielding $4.9 \mathrm{mg}$. This sample was purified using a $1 \mathrm{~mL}$ DIOL column eluting the column with $3 \mathrm{~mL}$ volumes of $50 \%$ hexanes in ethyl acetate which afforded oxazolidinone ND5_83A (157, $2.8 \mathrm{mg}$, 93\% yield).

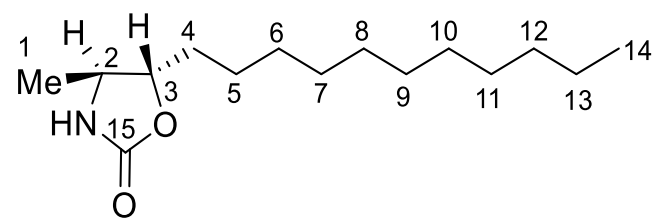

157

Oxazolidinone (157): $[\alpha]^{25} \mathrm{D}-43.3\left(c\right.$ 0.18, $\left.\mathrm{CHCl}_{3}\right)$; IR $v_{\max } 3285,2922,2853,1747$, $1541 \mathrm{~cm}^{-1} ;{ }^{1} \mathrm{H} \mathrm{NMR}\left(\mathrm{CDCl}_{3}, 600 \mathrm{MHz}\right) \delta 5.18(1 \mathrm{H}, \mathrm{s}, \mathrm{NH}), 4.09(1 \mathrm{H}, \mathrm{ddd}, J=7.9,6.4$, $4.8 \mathrm{~Hz}, \mathrm{H}-3), 3.57$ (1H, quin, $J=6.2 \mathrm{~Hz}, \mathrm{H}-2), 1.71(1 \mathrm{H}, \mathrm{m}, \mathrm{H}-4 \mathrm{~b}), 1.62$ (1H, m, H-4a), 1.48 (1H, m, H-5b), 1.37 (1H, m, H-5a), 1.28 (2H, m, H-13), 1.27 (3H, d, J=6.2 Hz, H-1), 1.26 (12H, m, H-6 to H-11), 1.25 (2H, m, H-12), 0.88 (3H, t, J = 7.0 Hz, H-14); ${ }^{13} \mathrm{C} \mathrm{NMR}\left(\mathrm{CDCl}_{3}, 150 \mathrm{MHz}\right) \delta 158.97$ (C-15), 84.4 (C-3) 53.7 (C-2), 34.3 (C-4), 32.1 (C-12), 29.76-29.46 (C-6 to C-11), 25.0 (C-5), 22.84 (C-13), 20.82 (C-1) 14.3 (C-14); HRESIMS $m / z 256.2264[\mathrm{M}+\mathrm{H}]^{+}\left(\right.$calcd for $\mathrm{C}_{15} \mathrm{H}_{30} \mathrm{NO}_{2}, 256.2271$ )

\subsubsection{Acetylation}

3-Epi-xestoaminol C (10 mg) was reacted with a 1:1 mixture of pyridine and acetic anhydride $(3 \mathrm{~mL})$ for $3 \mathrm{~h}$ at room temp and monitored via TLC. The reaction evaporated in vacuo and dissolved in DCM $(20 \mathrm{~mL})$ following which the organic layer was partitioned against $1 \mathrm{M} \mathrm{HCl}, 1 \mathrm{M} \mathrm{NaOH}$ and $\mathrm{H}_{2} \mathrm{O}$, yielding $7 \mathrm{mg}$ of diacetyl-3-epixestoaminol C (158, 70\% yield). 


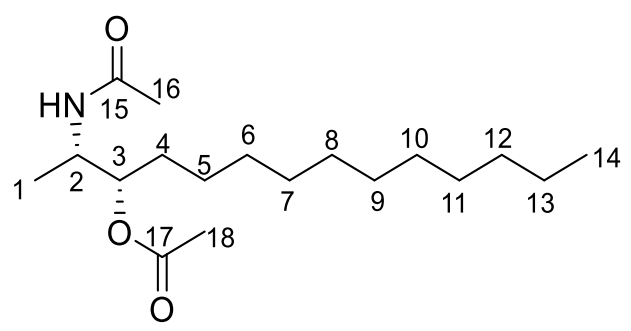

158

Diacetyl-3-epi-xestoaminol C (158): $[\alpha]^{25}$ D -23.0 (c 0.1, $\left.\mathrm{CHCl}_{3}\right)$; IR $v_{\max } 3297,2923$, 2854, 1740, 1652, 1460, $1373 \mathrm{~cm}^{-1} ;{ }^{1} \mathrm{H}$ NMR $\left(\mathrm{CDCl}_{3}, 600 \mathrm{MHz}\right) \delta 5.53(1 \mathrm{H}, \mathrm{d}, J=9.3$ $\mathrm{Hz}, \mathrm{NH}), 4.85$ (1H, ddd, $J=7.6,5.9,4.1 \mathrm{~Hz}, \mathrm{H}-3), 4.20(1 \mathrm{H}, \mathrm{dqd}, J=9.3,6.8,4.1 \mathrm{~Hz}$, H-2), 2.09 (3H, s, OAc, H-18), 1.99 (3H, s, NAc, H-16), 1.52 (2H, m, H-4), 1.28 (2H, m, H-13), 1.27 (2H, m, H-5) 1.25 (2H, m, H-12) 1.24 (12H, m, H-6 to H-11), 1.10 (3H, d, J $=6.8 \mathrm{~Hz}, \mathrm{H}-1), 0.87(3 \mathrm{H}, \mathrm{t}, J=7.0 \mathrm{~Hz}, \mathrm{H}-14) ;{ }^{13} \mathrm{C} \mathrm{NMR}\left(\mathrm{CDCl}_{3}, 150 \mathrm{MHz}\right) \delta 171.1(\mathrm{C}-$ 17), 169.6 (C-15), 76.6 (C-3), 47.4 (C-2), 32.1 (C-12), 31.7 (C-4), 29.78-29.5 (C-6 to C11), 25.4 (C-5), 23.6 (C-16), 22.8 (C-13), 21.2 (C-18), 18.7 (C-1), 14.3 (C-14); HRESIMS $m / z, 314.2693[\mathrm{M}+\mathrm{H}]^{+}\left(\right.$calcd for $\mathrm{C}_{18} \mathrm{H}_{36} \mathrm{NO}_{3}, 314.2690$ )

\subsubsection{Monodeacetylation}

Diacetyl-3-epi-xestoaminol C (158, $7 \mathrm{mg})$ was reacted with $5 \mathrm{mg}$ of $\mathrm{KCO}_{3}$ in $\mathrm{MeOH}(3$ $\mathrm{mL}$ ) at $0{ }^{\circ} \mathrm{C}$ for $30 \mathrm{~min}$ followed by room temperature for $16 \mathrm{~h}$. The reaction was monitored via TLC (10\% MeOH 90\% DCM), following which the reaction mixture was evaporated and re-dissolved in EtOAc $(15 \mathrm{~mL})$. This was partitioned against $\mathrm{H}_{2} \mathrm{O}$ three times to yield $4.7 \mathrm{mg}$ of $\mathrm{N}$-acetyl-3-epi-xestoaminol C (159,67\% yield).

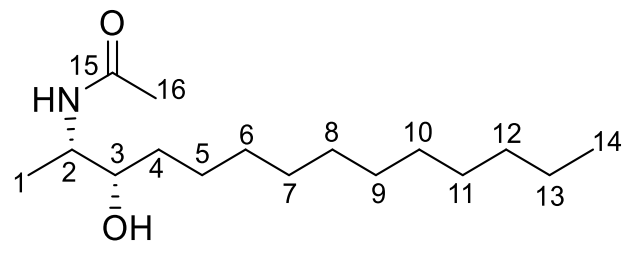

159

$N$-Acetyl-3-epi-xestoaminol C (159): $[\alpha]^{25}$ D -6.3 (c 0.16, $\left.\mathrm{CHCl}_{3}\right)$; IR $v_{\max } 3477,2924$, 2854, $1652 \mathrm{~cm}^{-1} ;{ }^{1} \mathrm{H}\left(\mathrm{CDCl}_{3}, 600 \mathrm{MHz}\right) \delta 5.68(1 \mathrm{H}, \mathrm{d}, J=8.54 \mathrm{~Hz}, \mathrm{NH}), 3.97(1 \mathrm{H}, \mathrm{dqd}$, $J=8.6,6.8,3.2 \mathrm{~Hz}, \mathrm{H}-2), 3.53(1 \mathrm{H}, \mathrm{q}, J=4.4,3.7 \mathrm{~Hz}, \mathrm{H}-3), 2.00$ (3H, s, NAc, H-16), 1.44 (1H, m, H-4), 1.41 (1H, m, H-5b), 1.34 (1H, m, H-5a), 1.29 (2H, m, H-13), 1.25 
(14H, m, H-6 to H-12), 1.19 (3H, d, $J=6.5 \mathrm{~Hz}, \mathrm{H}-1), 0.88(3 \mathrm{H}, \mathrm{t}, J=7 \mathrm{~Hz}, \mathrm{H}-14) ;{ }^{13} \mathrm{C}$ $\left(\mathrm{CDCl}_{3}, 150 \mathrm{MHz}\right) \delta 170.3(\mathrm{C}-15), 75.0$ (C-3), 49.1 (C-2), 34.6 (C-4), 32.1 (C-12), 29.8-29.5 (C-6 to C-11), 25.8 (C-5), 23.7 (C-16), 22.9 (C-13), 18.5 (C-1), 14.3 (C-12) HRESIMS $m / z, 272.2581[\mathrm{M}+\mathrm{H}]^{+}$(calcd for $\mathrm{C}_{16} \mathrm{H}_{34} \mathrm{NO}_{2}, 272.2584$ )

\subsubsection{R and S- Mosher's esters}

Two aliquots of $\mathrm{N}$-acetyl-3-epi-xestoaminol C (159) (1.5 $\mathrm{mg}$ each) were treated with EDCI (7.3 mg, 8.6 equiv) and DMAP (1.41 mg, 2.1 equiv) dissolved in dry DCM under argon. To each flask $9.02 \mathrm{mg}$ aliquots of either $(R \quad)-(+)$ - $\alpha$-methoxy- $\alpha$ trifluoromethylphenylacetic acid or $(S)-(-)-\alpha$-methoxy- $\alpha$-trifluoromethylphenylacetic (MTPA) (7 equivalents) acid were added. Reactions were monitored via TLC over $22 \mathrm{~h}$ (10\% $\mathrm{MeOH}, 90 \% \mathrm{DCM})$. Upon completion, reaction mixtures were washed with $0.01 \mathrm{M} \mathrm{HCl}$ followed by $0.01 \mathrm{M} \mathrm{NaOH}$ and finally $\mathrm{H}_{2} \mathrm{O}$ before performing HPLC purification of the $R(\mathbf{1 6 0})$ and $S$ esters (161) using a C18 analytical column (Phenomenex Prodigy, 4.6 x $250 \mathrm{~mm}, 5 \mu \mathrm{m}$ particle size) at a flow rate of $1 \mathrm{~mL} / \mathrm{min}(5$ $\min$ at $30 \% \mathrm{MeCN}, 15 \mathrm{~min}$ ramp to $100 \% \mathrm{MeCN}, 5 \mathrm{~min}$ at $100 \% \mathrm{MeCN}, 2 \mathrm{~min}$ ramp down to $30 \% \mathrm{MeCN}, 5 \mathrm{~min}$ hold at $30 \% \mathrm{MeCN})$ to give $R(\mathbf{1 6 0}, 0.6 \mathrm{mg})$ and $S(\mathbf{1 6 1}, 1.2$ mg) esters.

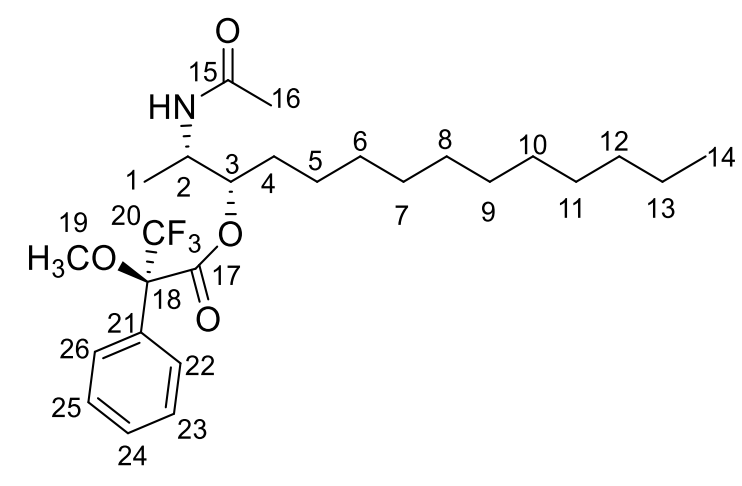

160

$R$-ester (160): $[\alpha]^{25} \mathrm{D} 0\left(c 0.04, \mathrm{CHCl}_{3}\right)$; IR $v_{\max } 3378,2924,2854,1746,1651 \mathrm{~cm}^{-1} ;{ }^{1} \mathrm{H}$ NMR (CD $\left.{ }_{3} \mathrm{OD}, 600 \mathrm{MHz}\right) \delta 7.55$ (2H, m, H-24), 7.45 (2H, m, H-22), 7.44 (1H, m, H23), $5.5(1 \mathrm{H}, \mathrm{s}, \mathrm{NH}), 5.11(1 \mathrm{H}, \mathrm{ddd}, J=7.6,5.6,4.6 \mathrm{~Hz}, \mathrm{H}-3), 4.17(1 \mathrm{H}, \mathrm{qd}, J=6.9,5.5$ Hz, H-2), 3.54 (3H, s, OMe, H-19), 1.83 (3H, s, NAc, H-16), 1.64 (2H, m, H-4), 1.34 (3H, m, H-5b, H-6), 1.31 (2H, m, H-5a, H-13b), 1.29 (1H, m, H-13a), 1.28 (12H, m, H- 
7 to $\mathrm{H}-12), 0.99(3 \mathrm{H}, J=\mathrm{d}, 6.9 \mathrm{~Hz}), 0.90(3 \mathrm{H}, \mathrm{t}, J=7 \mathrm{~Hz}, \mathrm{H}-14) ;{ }^{13} \mathrm{C} \mathrm{NMR}\left(\mathrm{CD}_{3} \mathrm{OD}\right.$, $150 \mathrm{MHz}) \delta 172.7$ (C-15), 167. 5 (C-17), 133.4 (C-18), 130.9 (C-23/25), 129.5 (C-24), 128.7 (C-22/26), 125.8 (C-21), 86.1 (C-20), 79.7 (C-3), 56.1 (C-19), 47.7 (C-2), 33.1 (C-12), 31.5 (C-4), 30.75-30.4, 26.1 (C-6 to C-11), 30.48 (C-5), 23.8 (C-13), 22.6 (C16), 16.8 (C-1), 14.5 (C-14); HRESIMS $m / z 488.2979[\mathrm{M}+\mathrm{H}]^{+}$(calcd for $\mathrm{C}_{26} \mathrm{H}_{41} \mathrm{~F}_{3} \mathrm{NO}_{4}$, 488.2982).

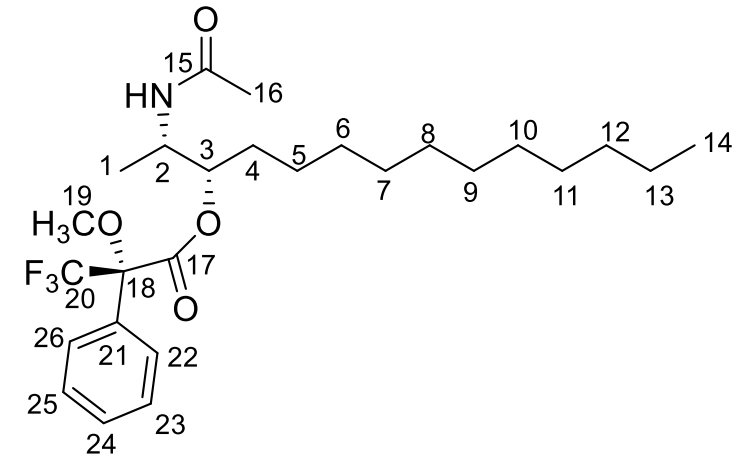

161

$S$-ester (161): $[\alpha]^{25}$ D -17.5 (c 0.13, $\left.\mathrm{CHCl}_{3}\right)$; IR vmax_3335, 2925, 2854, 1746, $1651 \mathrm{~cm}^{-1}$; ${ }^{1} \mathrm{H}$ NMR (CD $\left.3 \mathrm{OD}, 600 \mathrm{MHz}\right) \delta 7.55$ (2H, m, H-24), 7.44 (3H, m, H-22, H-23), 5.5 (1H, s, NH), $5.11(1 \mathrm{H}$, ddd, $J=8.0,6.4,4.0 \mathrm{~Hz}, \mathrm{H}-3), 4.2(1 \mathrm{H}$, quin, $J=6.8 \mathrm{~Hz}, \mathrm{H}-2), 3.55$ (3H, s, OMe H-19), 1.9 (3H, s, NAc, H-16), 1.59 (1H, m, H-4b), 1.54 (1H, m, H-4a), 1.32 (1H, m, H-13b), 1.29 (1H, m, H-13a), 1.28 (2H, m, H-12), 1.27-1.19 (12H, m, H-6 to $\mathrm{H}-11), 1.15$ (2H, m, H-5), $1.13(3 \mathrm{H}, \mathrm{d}, J=6.9 \mathrm{~Hz}, \mathrm{H}-1), 0.90$ (3H, t, $J=6.9 \mathrm{~Hz}, \mathrm{H}-14) 1$; ${ }^{13} \mathrm{C}$ NMR (CD $\left.3 \mathrm{OD}, 150 \mathrm{MHz}\right) \delta 172.7$ (C-15), 167.5 (C-17), 133.5 (C-18), 130.9 (C23/25), 129.5 (C-24), 128.5 (C-22/26), 125.8 (C-21), 85.9 (C-20), 79.5 (C-3), 56.2 (C19), 48.1 (C-2), 33.1 (C-12), 31.3 (C-4), 30.74-30.3 (C-6 to C-11), 25.7 (C-5), 23.8 (C13), 22.7 (C-16), 17.0 (C-1), 14.5 (C-14); HRESIMS m/z $488.2979[\mathrm{M}+\mathrm{H}]^{+}$(calcd for $\mathrm{C}_{26} \mathrm{H}_{41} \mathrm{~F}_{3} \mathrm{NO}_{4}, 488.2982$ )

\subsubsection{Sensitive mutant screen}

A random mutant library with $32 \times 384$-well plates comprising of 7680 Tn611 M. smegmatis transposon mutants was generated by Richard Campen (VUW). These mutants were pinned into corresponding assay plates containing $100 \mu \mathrm{L}$ LB Kan 20 
(section 2.1.1.2) and incubated for $72 \mathrm{~h}$, these plates were then sub-inoculated into corresponding LB Kan 20 plates for a further $24 \mathrm{~h}$, following which the 24 hour cultures were pinned into plates with LB $\mathrm{Kan}_{20}$ and $2 \mu \mathrm{g} / \mathrm{mL}$ of 3-epi-xestoaminol C (156). These plates were incubated for $48 \mathrm{~h}$ after which $20 \mu \mathrm{L}$ of $0.03 \%$ resazurin (w/v) was added to plates and measured for fluorescence after $2 \mathrm{~h}$ or colour change was visible (from blue to purple/pink).

Hits were mutants that identified growth sensitivity, \% growth was measured for each well. Using Microsoft Excel the wells with the most sensitivity to growth were ranked to identify the top 120 mutants. These mutants were split into half and were picked from library wells and grown in $100 \mu \mathrm{L}$ volumes in two 96-well plates. After culturing plates for $72 \mathrm{~h}, 70 \mu \mathrm{L}$ of $80 \%$ glycerol was added to these plates to generate freezer stocks of these cultures to a final concentration of $33 \%$ glycerol. For screening, the glycerol plates were pinned into $100 \mu \mathrm{L}$ of $\mathrm{LB} \mathrm{Kan}_{20}$ to grow for $48 \mathrm{~h}$, these cultures were sub-inoculated into fresh $100 \mu \mathrm{L} \mathrm{LB} \mathrm{Kan} 20$ plates and grown for a further $24 \mathrm{~h}$. These cultures were pinned into plates containing LB $\mathrm{Kan}_{20}$ and $2 \mu \mathrm{g} / \mathrm{mL}$ of 3-epi-xestoaminol C (156) and incubated for $48 \mathrm{~h}$ after which OD measurements as well resazurin measurements were taken. From this screening out of the top 60 hits, 17 hits were validated in dose response assays and from the second 60 hits, 14 hits were validate in dose response assays.

\subsubsection{Resistant mutant generation and screen protocol}

A pool of random Tn611 mutants was generated using a modification of the methods described by Gilhot and Perez [129, 308]. In brief, these mutants were generated as follows: a pcG70 vector carrying the Tn611 transposon was electroporated into M. smegmatis $\mathrm{mc}^{2} 155$ following which cells were plated onto LB $\mathrm{Kan}_{20}$ plates at $30{ }^{\circ} \mathrm{C}$. Colonies were picked and grown in LB $\mathrm{Kan}_{20}$ at $30{ }^{\circ} \mathrm{C}$ for $48 \mathrm{~h}$ at $200 \mathrm{RPM}$. This allows for the replication and generation of a large pool of transposon mutants. These cultures were transferred to a $41{ }^{\circ} \mathrm{C}$ incubator for $24 \mathrm{~h}$, this ceases transposition events. Glycerol was then added to cultures to achieve a final concentration of $30 \%$ in order to generate freezer stocks of mutants. For the control strain the plasmid pRC10 was electroporated into M. smegmatis $\mathrm{mc}^{2} 155$ and glycerol stocks were made. Following 
electroporation, colony forming units (CFUs) were counted in order to have an accurate gauge of the number of cells plated out in a $100 \mu \mathrm{L}$ aliquot of cells.

Agar plates were made up to either $10,7.5$ of $3.75 \mu \mathrm{g} / \mathrm{mL}$ of 3 -epi-xestoaminol C (156), following which $100 \mu \mathrm{L}$ of transposon mutants were plated out. This was performed in duplicate. Control strains of pRC10 were also plated out.

\subsubsection{G2-M block assay}

In brief, using 24-well plates 1A9 cells were diluted to a final cell concentration of $1 \mathrm{x}$ $10^{5}$ in a final volume of $500 \mu \mathrm{L}$. The drug concentrations used were $\mathrm{IC}_{99}, \mathrm{IC}_{50}$ and $\mathrm{IC}_{10}$ to allow for analysis at concentrations at which complete death, 50\% cell death and a concentration at which no major inhibition occurs. Caution was taken not to exceed the final DMSO concentration of $0.5 \%$ per well to avoid inhibition of cell growth due to solvent effects. Cells were incubated with drug over $16 \mathrm{~h}$ at $37{ }^{\circ} \mathrm{C} 5 \% \mathrm{CO}_{2}$ before labelling cells with propidium iodide and performing fluorescence activated cell sorting (FACS) analysis.

\subsubsection{S. cerevisiae bioassay methods}

\subsubsection{Yeast strains, media and chemicals.}

The Saccharomyces cerevisiae deletion library (Open Biosystem) was generated from

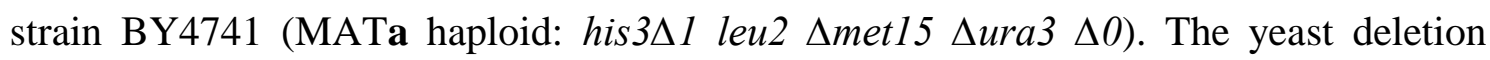
library was stored in $-80{ }^{\circ} \mathrm{C}$ and grown in yeast extract $(10 \mathrm{~g} / \mathrm{L})$-peptone $(20 \mathrm{~g} / \mathrm{L})$ dextrose (20 g/L) (YPD) agar. 3-Epi-xestoaminol C (156), was dissolved in DMSO to give a $500 \mu \mathrm{g} / \mathrm{mL}$ working stock before use.

\subsubsection{Screening for 3-epi-xestoaminol C sensitivity and resistance in liquid medium.}

Replications and inoculation of yeast cells were run using the Singer RoToR HDA ${ }^{\odot}$. Yeast knockout mutants were tested in 96-well plates containing Synthetic Complete (SC) $(1.7 \mathrm{~g} / \mathrm{L}$ yeast nitrogen base, $1 \mathrm{~g} / \mathrm{L}$ monosodium glutamate, $2 \mathrm{~g} / \mathrm{L}$ amino acid mixture) broth media in each well. Yeast were incubated for $20 \mathrm{~h}$ at $30{ }^{\circ} \mathrm{C}$ and then inoculated into 96-well plates containing $200 \mu \mathrm{L} \mathrm{SC}$ with $0.8 \mu \mathrm{g} / \mathrm{mL}$ final concentration 
of 3-epi-xestoaminol C (156), and the appropriate volume of DMSO (solvent control). Plates were sealed with gas permeable seals and incubated for $16 \mathrm{~h}$ at $30{ }^{\circ} \mathrm{C}$. Optical density (OD) was measured at $590 \mathrm{~nm}\left(\mathrm{OD}_{590}\right)$ using Perkin Elmer EnVision 2102 Multilabel Reader, the $16^{\text {th }}$ hour OD 590 was normalised to the $\mathrm{OD}_{590}$ at $0^{\text {th }}$ hour. The screen was done in triplicate, with the \% residual growth of drug treated cells measured in comparison to DMSO treated cells using Microsoft Excel.

Hits were selected using a $t$ test with a Bonferroni correction adjusting the $p$ value to 0.017. Using this selection criterion, sensitive strains that displayed less than $70 \%$ residual growth and resistant strains that had greater than $130 \%$ growth in comparison to DMSO controls were chosen for validation. Using the same selection criterion, these hits were validated again in triplicate to identify the hits for generating a network map. 


\subsection{Results}

\subsubsection{Bioassay data}

Using the methodology described in section 2.6, the results of the dose-response assays for 3-epi-xestoaminol C (156) are listed below. Graphs were generated using GraphPad Prism v.5.0. Resazurin dye was used to a measure bacterial metabolism after each assay was run, and fluorescence measurements (RFU) were used as a measure of growth. OD was used as measure of growth for the HL-60 and HEK MTT assays. OD measurements were also used for all $S$. cerevisiae assays. Where possible $\mathrm{IC}_{50}$ values have been listed, and as stated earlier as sigmoidal curves were not achievable for all data, MIC 90 values have been listed as well.

\subsubsection{Antimicrobial assays}

Table 6.1 3-Epi-xestoaminol C SAR work

\begin{tabular}{lc}
\hline Compound & $\begin{array}{c}\text { MIC }_{90}(\mu \mathrm{g} / \mathbf{m L}) \text { in M. tuberculosis } \\
\text { H37Ra }\end{array}$ \\
\hline 3-epi-xestoaminol C (156) & 15 \\
Oxazolidinone (157) & $\geq 60$ \\
Diacetyl-3-epi-xestoaminol C (158) & IA \\
$N$-acetyl-3-epi-xestoaminol C (159) & IA \\
$R$-Mosher's ester (160) & IA \\
$S$-Mosher's ester (161) & IA
\end{tabular}

Bioactivity of derivatives of 3-epi-xestoaminol C against M. tuberculosis H37Ra using growth inhibition assays. IA: inactive at the highest concentration tested $(60 \mu \mathrm{g} / \mathrm{mL})$. 
3-epi-xestoaminol C vs. M. tuberculosis H37Ra

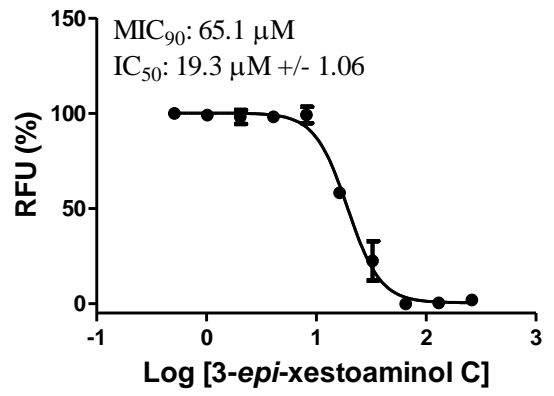

A

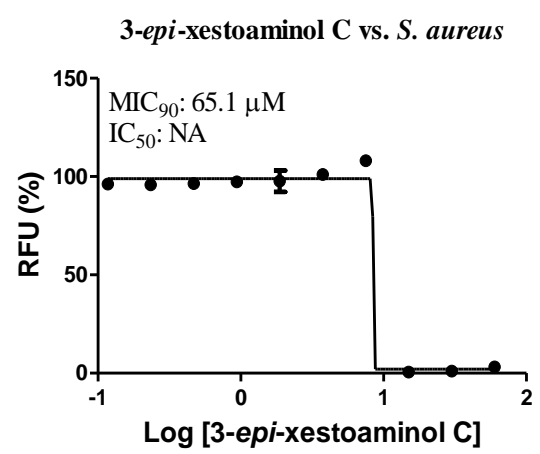

C

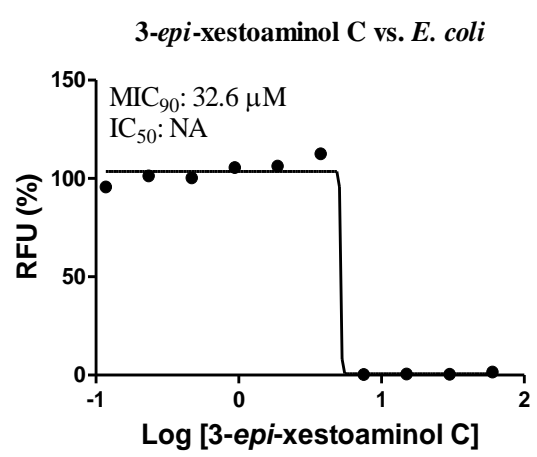

B

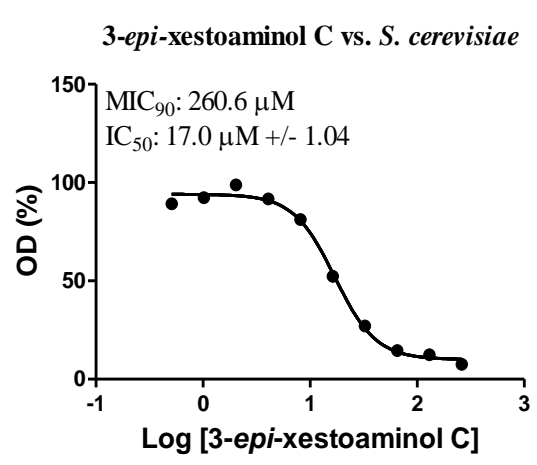

D

Figure 6.1 Antimicrobial activity of 3-epi-xestoaminol C (156).

Antimicrobial activity of 3-epi-xestoaminol C (156) against a range of microorganisms, using growth inhibition assays, compound has been diluted two fold before addition of cultures. Assay durations ranged from $16 \mathrm{~h}$ for E. coli, S. aureus and S. cerevisiae to 7 days for M. tuberculosis H37Ra. Percentage growth signal inhibition on the Y-axis is relative to controls measured. NA: not achievable. $(n=1$, triplicate) 


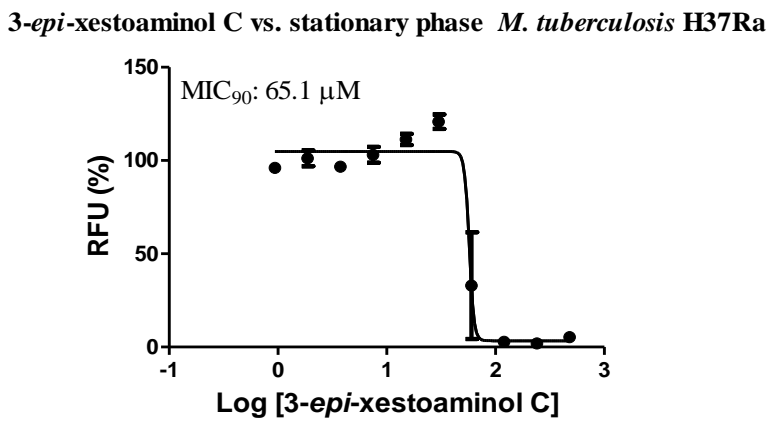

Figure 6.2 Stationary phase assay

Inhibitory effects of 3-epi-xestoaminol C (156) against stationary phase cultures of $M$. tuberculosis H37Ra. Compound was serially diluted two fold in PBS before 30 day old cultures washed and re-suspended in PBS were added to plates. ( $\mathrm{n}=1$, triplicate)

\subsubsection{Mammalian cell line assays}

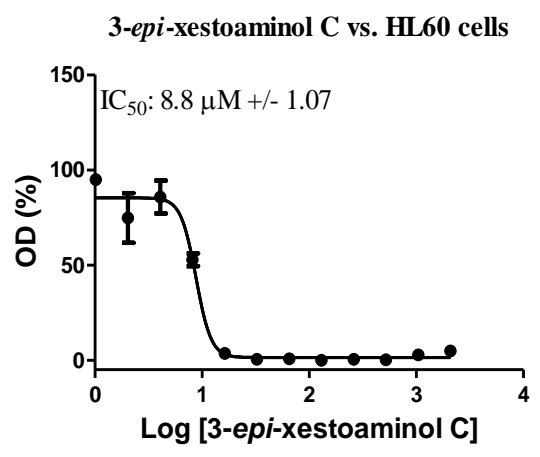

A

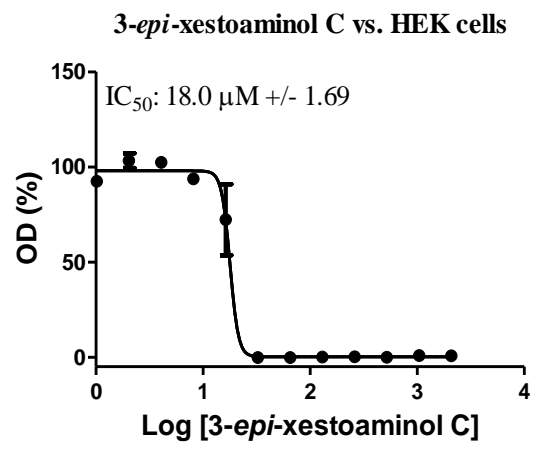

B

Figure 6.3 3-epi-xestoaminol C (156) vs. mammalian cell lines

The bioactivity of 3-epi-xestoaminol C (156) in HL-60 (A) and HEK cell lines (B). The HEK cell line has been used as a representative of general cytotoxicity. OD was used as a measure of growth. ( $\mathrm{n}=1$, duplicate) 


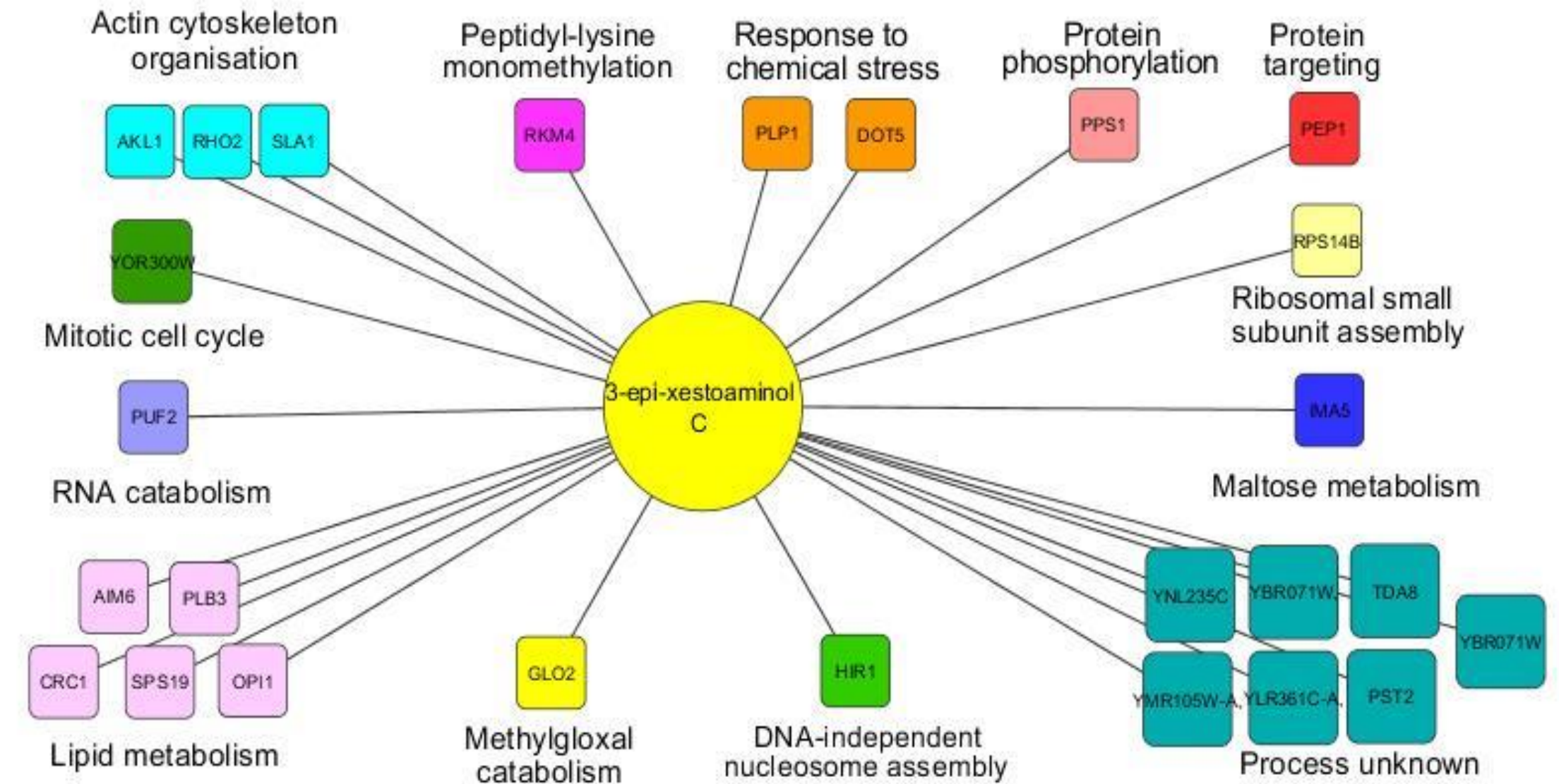

Figure 6.4 Network map of hits from yeast chemical genetics screening of 3-epi-xestoaminol C (156). 


\subsection{Discussion}

\subsubsection{Fractionation and isolation}

PTN2_93D, a brown algae identified as Xiphophora chondrophylla, was observed as being active against all three species of bacteria screened using the VUW MNP library. As the crude extract displayed similar levels of activity against M. tuberculosis H37Ra and M. smegmatis, M. smegmatis was used for the subsequent bioassays. As the $40 \%$ acetone fraction from the initial HP20 fractionation had the highest mass and displayed the best $\mathrm{MIC}_{90}$ of $50 \mu \mathrm{g} / \mathrm{mL}$, it was taken to the next stage of purification. Half of the mass of this fraction was separated using HP20ss and based on the activity profiles of test tubes 43-46 (15.6 $\mu \mathrm{g} / \mathrm{mL}, 17.8 \mathrm{mg})$ and 47-54 $(15.6 \mu \mathrm{g} / \mathrm{mL}, 78.2 \mathrm{mg})$, they were further pursued. Attempts to purify the $78.2 \mathrm{mg}$ fraction using reversed phase (C18) HPLC did not achieve any separation. Guided by bioassays, the $17.8 \mathrm{mg}$ fraction was separated a further three times using LH20 size exclusion chromatography. Ultimately using a DIOL column for the last purification led to the identification of an amino alcohol 3-epi-xestoaminol C (156) as the compound responsible for bioactivity.

In order to isolate more of the active compound to elucidate its relative and absolute configurations, a more refined isolation scheme was employed to separate the second half of the $40 \%$ acetone fraction (Scheme 6.2). Testing the purified compound using the optimised method identified a consistent $\mathrm{MIC}_{90}$ of $15 \mu \mathrm{g} / \mathrm{mL}$ in all microbes tested (Figure 6.1).

\subsubsection{Structural elucidation}

HRESIMS analysis indicated an $[\mathrm{M}+\mathrm{H}]^{+}$adduct ion at $\mathrm{m} / \mathrm{z}, 230.2467$, suitable for the formula $\mathrm{C}_{14} \mathrm{H}_{32} \mathrm{NO}$ (calcd for 230.2478), and having no double bond equivalents. The ${ }^{1} \mathrm{H},{ }^{13} \mathrm{C}$ and multiplicity-edited HSQC NMR spectra in $\mathrm{CD}_{3} \mathrm{OD}$ (Table 6.2) revealed 32 protons attached to 14 carbons, including two methyls ( $\delta_{\mathrm{C}} 16.1$ and 14.5 ), ten methylenes $\left(\delta_{\mathrm{C}} 34.7,33.1,30.8-30.5,26.4,23.8\right)$ an oxymethine $\left(\delta_{\mathrm{C}} 73.2\right)$ and an aminomethine $\left(\delta_{\mathrm{C}} 53.5\right)$. 
The connectivity from $\mathrm{C}-1$ to $\mathrm{C}-5$ was elucidated using a continuous sequence of COSY correlations from the terminal methyl doublet $\left(\mathrm{C}-1: \delta_{\mathrm{C}} 16.1, \delta_{\mathrm{H}} 1.27\right)$ to the aminomethine $\left(\mathrm{C}-2: \delta_{\mathrm{C}} 53.5, \delta_{\mathrm{H}} 3.09\right)$, to the oxymethine $\left(\mathrm{C}-3: \delta_{\mathrm{C}} 73.2, \delta_{\mathrm{H}} 3.44\right)$, and to the C-4 methylene $\left(\mathrm{C}-4: \delta_{\mathrm{C}} 34.7, \delta_{\mathrm{H}} 1.40,1.56\right)$. HMBC correlations from H-3, H-4a and $\mathrm{H}-4 \mathrm{~b}$ to $\mathrm{C}-5\left(\delta_{\mathrm{C}} 26.4, \delta_{\mathrm{H}} 1.39,1.54\right)$ provided further evidence for this proposed substructure (Figure 6.5).

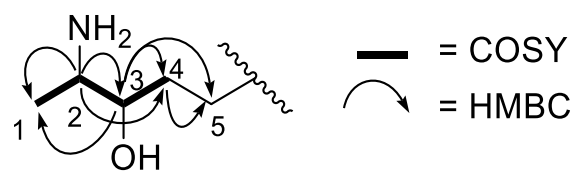

\section{Figure 6.5 Key COSY and HMBC correlations establishing substructure 1}

Similarly, the connectivity from C-14 to C-12 was established based on the COSY correlations from the methyl triplet $\left(\mathrm{C}-14: \delta_{\mathrm{C}} 14.5, \delta_{\mathrm{H}} 0.91\right)$ to the methylenes $\mathrm{C}-13\left(\delta_{\mathrm{C}}\right.$ $\left.23.8, \delta_{\mathrm{H}} 1.31\right)$ and $\mathrm{C}-12\left(\delta_{\mathrm{C}} 33.1, \delta_{\mathrm{H}} 1.29\right)$ to provide a second substructure (Figure 6.6).

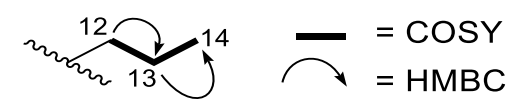

Figure 6.6 Key COSY and HMBC correlations establishing substructure 2

HMBC correlations into a methylene envelope $\left(\delta_{\mathrm{C}} 30.8-30.5, \delta_{\mathrm{H}} 1.30\right)$ as well as the number of carbons and hydrogens required by the molecular formula provided strong evidence for the proposed structure of a long alkyl chain connecting the two proposed sub-structures (Figure 6.7).

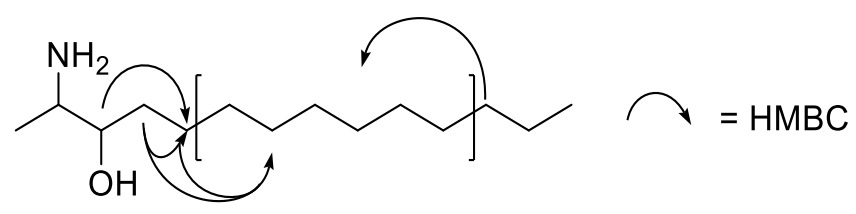

\section{Figure 6.7 Proposed planar structure}

To provide further evidence for the connection between the two termini, a 1D TOCSY experiment was performed in $\mathrm{CDCl}_{3}$. Irradiation of $\mathrm{H}-3\left(\delta_{\mathrm{H}} 3.65\right)$ and $\mathrm{H}_{2}-4 / 5\left(\delta_{\mathrm{H}}\right.$ $1.56 / 54)$ resulted in TOCSY transfer of energy to $\mathrm{H}_{3}-14\left(\delta_{\mathrm{H}} 0.88\right)$, while irradiation of $\mathrm{H}_{3}-14$ resulted in TOCSY transfer of energy to $\mathrm{H}-4 / 5$. This provided unequivocal evidence for the connectivity of the two proposed sub-structures of the amino alcohol (Figure 6.8) (for TOCSY spectra refer to Appendix D page 189). 


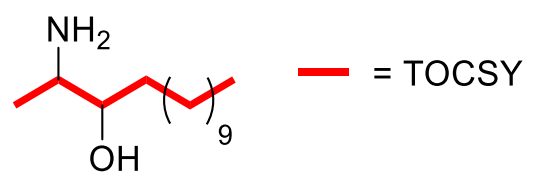

\section{Figure 6.8 TOCSY correlations}

From a literature search using MarinLit [210] and SciFinder ${ }^{\odot}$, the carbon connectivity was the same as the known amino alcohol xestoaminol C (109), first reported by Jiménez and Crews from a Xestospongia sp. sponge [284]. However, further analysis of the ${ }^{1} \mathrm{H}$ NMR spectrum of $156\left(\mathrm{CDCl}_{3}\right)$ identified several significant differences to that reported by Crews for 109 (156:1.38 ppm, $\mathrm{H}_{3}-1$; and 3.65 ppm, H-3; 109: 1.15 ppm, $\mathrm{H}_{3}-1 ; 3.95$ ppm, H-3) [284]. One possible explanation for this shift in resonance could be a different configuration, therefore these findings warranted further investigation into the compound isolated.

To identify the relative stereochemistry of the amino alcohol, it was reacted with 1,1' carbonyldiimidazole (CDI) to generate the oxazolidinone derivative (157). The coupling constant between $\mathrm{H}-2$ and H-3 was approximately $6.5 \mathrm{~Hz}$, diagnostic of a trans relationship between the protons of the five membered ring system [274]. In comparison a cis relationship would have a coupling constant of approximately $7.3 \mathrm{~Hz}$.

The trans relationship of these protons can be accounted for if the amine and hydroxyl group were syn to each other in the acyclic system. To confirm the trans relationship in the oxazolidinone, a 1D-NOE experiment was performed, irradiating H-1, H-2, H-3, H4a and H-4b. Irradiating H-3 enhanced H-1 while irradiation of H-4a and b led to the excitation of $\mathrm{H}-2$, confirming the trans relationship in the cyclic system. This therefore indicated that $\mathbf{1 5 6}$ has a syn relationship between $\mathrm{NH}_{2}-2$ and $\mathrm{OH}-3$, which differed from xestoaminol C (109) that has a trans relationship.

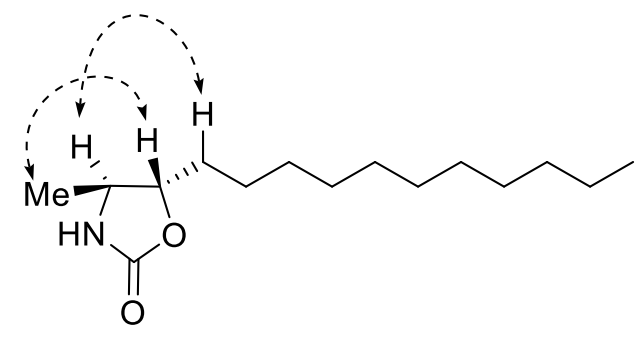

Figure 6.9 NOE correlations of oxazolidinone 157. 
With enough evidence to support a different relative configuration in $\mathbf{1 5 6}$ to $\mathbf{1 0 9}$, Mosher's method was used to solve the absolute configuration, which was carried out in three stages. Amino alcohol $\mathbf{1 5 6}$ was first treated in a 1:1 mixture of pyridine and acetic anhydride to acetylate the amine and hydroxyl groups, generating the diacetyl derivative (158). Following acetylation, the hydroxyl group was deprotected using base hydrolysis with $\mathrm{KCO}_{3}$ in $\mathrm{MeOH}$ to afford $\mathrm{N}$-acetyl-3-epi-xestoaminol $\mathrm{C}(\mathbf{1 5 9})$, which was treated with $R$ and $S$ - MTPA acids to yield the $R(\mathbf{1 6 0})$ and $S(\mathbf{1 6 1})$ Mosher's esters.

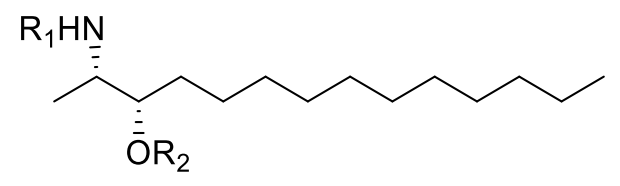

$158 \mathrm{R}_{1}=\mathrm{R}_{2}=\mathrm{Ac}$

$159 \mathrm{R}_{1}=\mathrm{Ac} \mathrm{R}_{2}=\mathrm{H}$

$160 \mathrm{R}_{1}=\mathrm{Ac} \mathrm{R}_{2}=R-\mathrm{MTPA}$

$161 \mathrm{R}_{1}=$ Ac $\mathrm{R}_{2}=S-\mathrm{MTPA}$

When analysing the changes in ${ }^{1} \mathrm{H}$ chemical shifts of the Mosher's esters in $\mathrm{CDCl}_{3}$, an interesting result was observed (Figure 6.10). Under normal circumstances, the $\Delta \delta$ shifts flanking the ester would either be solely positive or negative depending on the positioning of the phenyl ring. However, a mixture of shifts was observed at the amino terminal end of the compound (Figure 6.11). Hydrogen bonding between the carbonyl group of the ester and the amide proton may have caused a conformational change from that required to adequately study each side, as evidenced by the different couplings observed for H-3, indicated by different multiplicities (Figure 6.10). This proposed intramolecular bonding could directly affect the shielding and desheilding effects of MTPA, obscuring interpretation of data. 


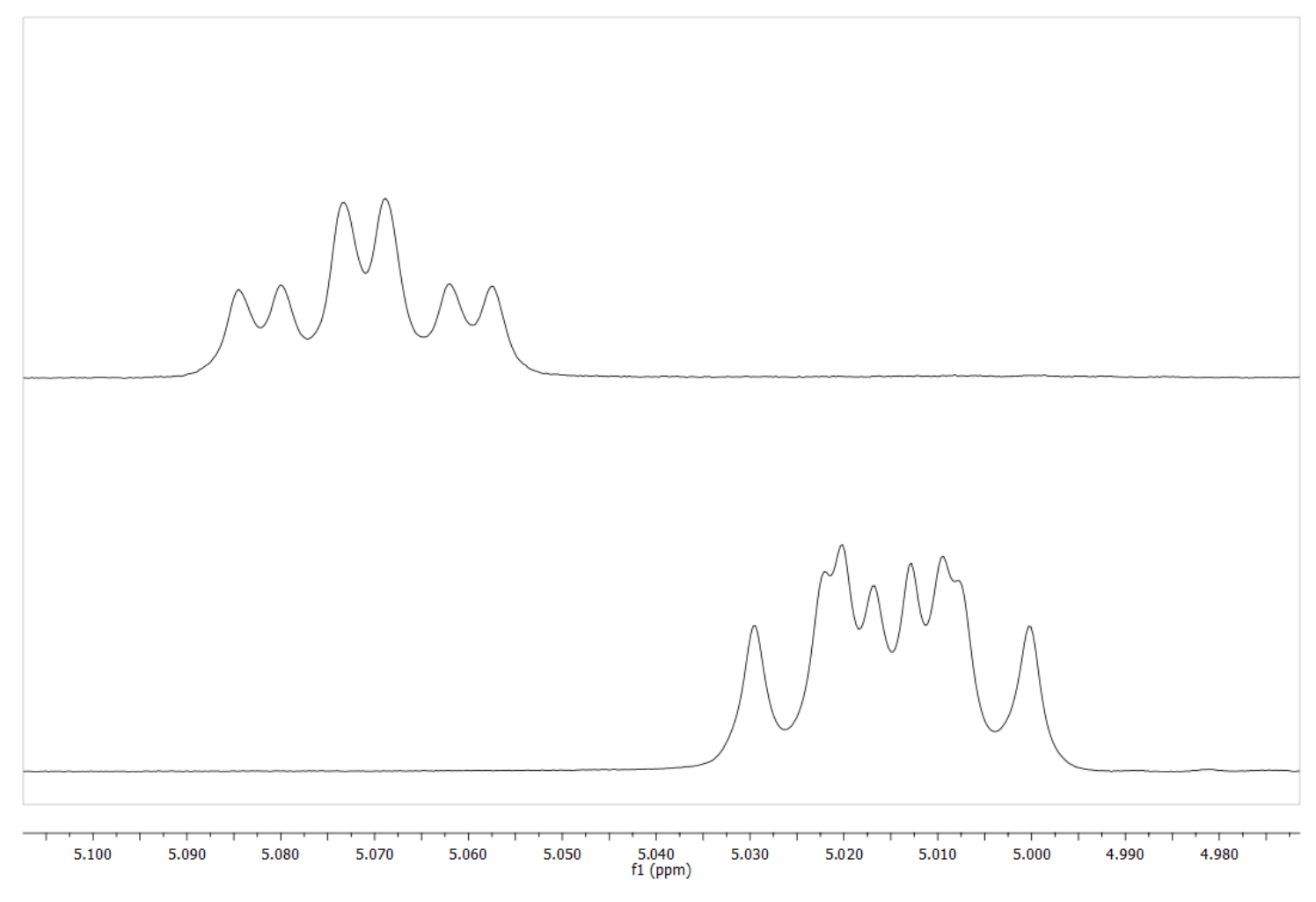

Figure 6.10 Change in multiplicity of the oxymethine ${ }^{1} \mathrm{H}$ NMR resonance for $R$ ester (160, top panel) and $S$-ester $\left(161\right.$, bottom panel) in $\mathrm{CDCl}_{3}(600 \mathrm{MHz})$.

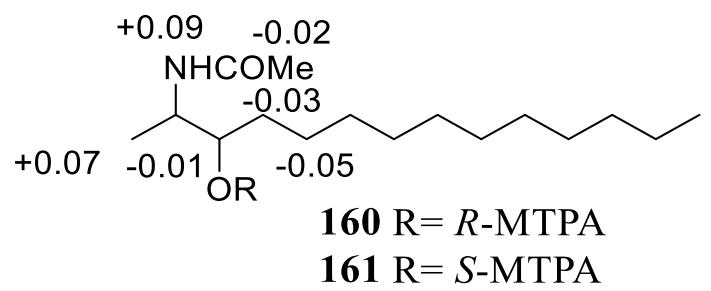

Figure 6.11 $\Delta \delta{ }^{1} \mathrm{H}$ NMR data of MTPA esters 160 and 161 in $\mathrm{CDCl}_{3}(600 \mathrm{MHz})$. 
In order to disrupt the effects of intramolecular hydrogen bonding, NMR analysis was performed in the polar deuterated solvent $\mathrm{CD}_{3} \mathrm{OD}$. As expected, the oxymethine resonance no longer varied between the $R$ and $S$-esters and both had roughly the same multiplicity (Figure 6.12). Furthermore, comparison of ${ }^{1} \mathrm{H}$ NMR data from the two esters indicated that the intramolecular bonding was no longer causing a misinterpretation of the data (Figure 6.15).

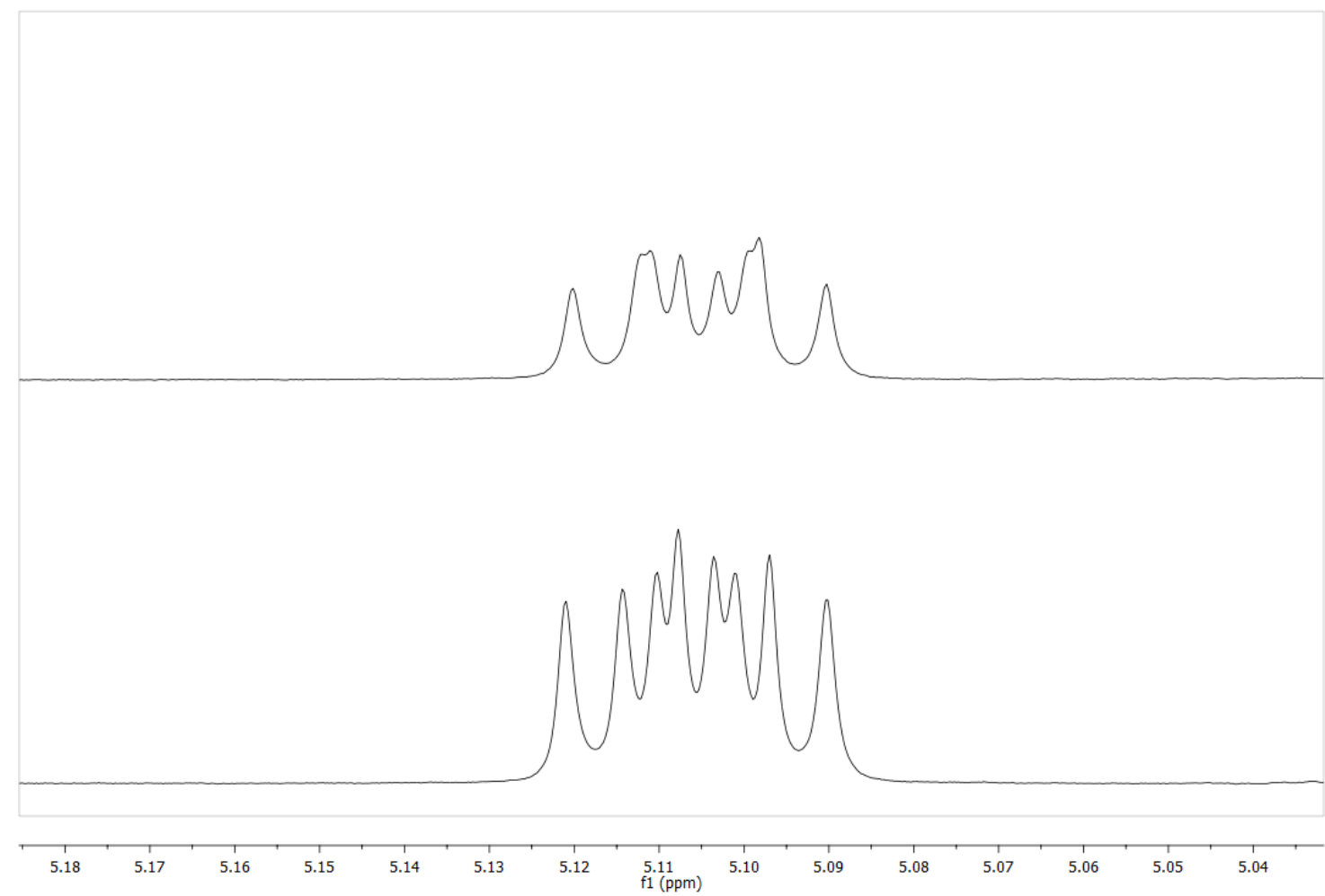

Figure 6.12 ${ }^{1} \mathrm{H}$ NMR oxymethine resonance for $R$-ester $(160$, top panel) and $S$-ester (161, bottom panel) in $\mathrm{CD}_{3} \mathrm{OD}(600 \mathrm{MHz})$.

Analysing the multiplicities in $\mathrm{CD}_{3} \mathrm{OD}$ (Figure 6.12), it can be observed that they match that of the $S$-ester in $\mathrm{CDCl}_{3}$ (Figure 6.10), and therefore it can be deduced that the $R$ ester was the cause of the issues in interpretation due to intramolecular bonding.

Using Mosher's method, and performing the analysis in the appropriate deuterated solvent provided unequivocal evidence for the absolute stereochemistry of 3-epi-xestoaminol $\mathrm{C}$. The $\Delta \delta$ values on either side of the ester were either solely positive or solely negative (Figure 6.13 ), and based on the $\Delta \delta$ shifts, it was possible to elucidate the absolute structure as $2-S$-aminotetradeca-3-S-ol. Figures 6.14 and 6.15 illustrate the shifts observed in the ${ }^{1} \mathrm{H}$ NMR spectra of the $R$ and $S$-esters. 


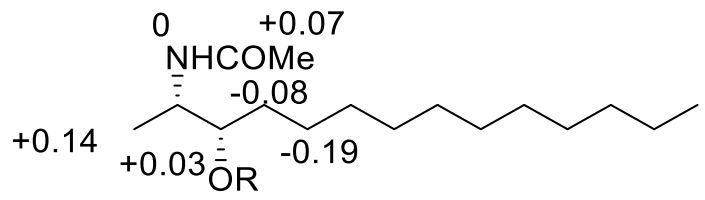

$160 \mathrm{R}=R-\mathrm{MTPA}$

$161 \mathrm{R}=S$-MTPA

Figure 6.13 $\Delta \delta{ }^{1} \mathrm{H}$ shifts between MTPA esters 160 and 161 of $N$-acetylated xestoaminol $\mathrm{C}$ in $\mathrm{CD}_{3} \mathrm{OD}$.

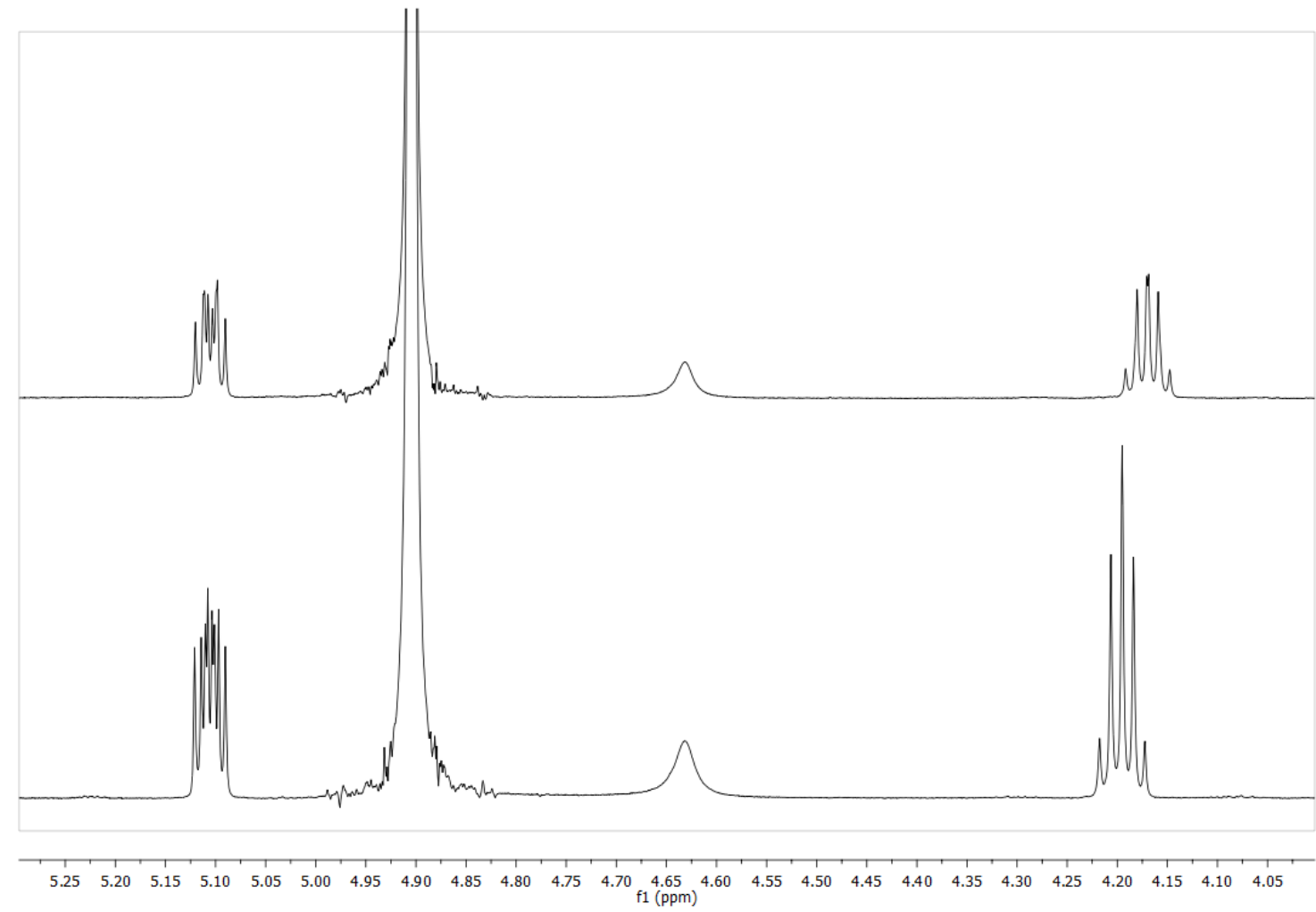

Figure 6.14 Oxy- and aminomethine region, ${ }^{1} \mathrm{H}$ NMR spectra for the $R$-ester $(160$, top panel) and $S$-ester (161, bottom panel) in $\mathrm{CD}_{3} \mathrm{OD}(600 \mathrm{MHz})$ 


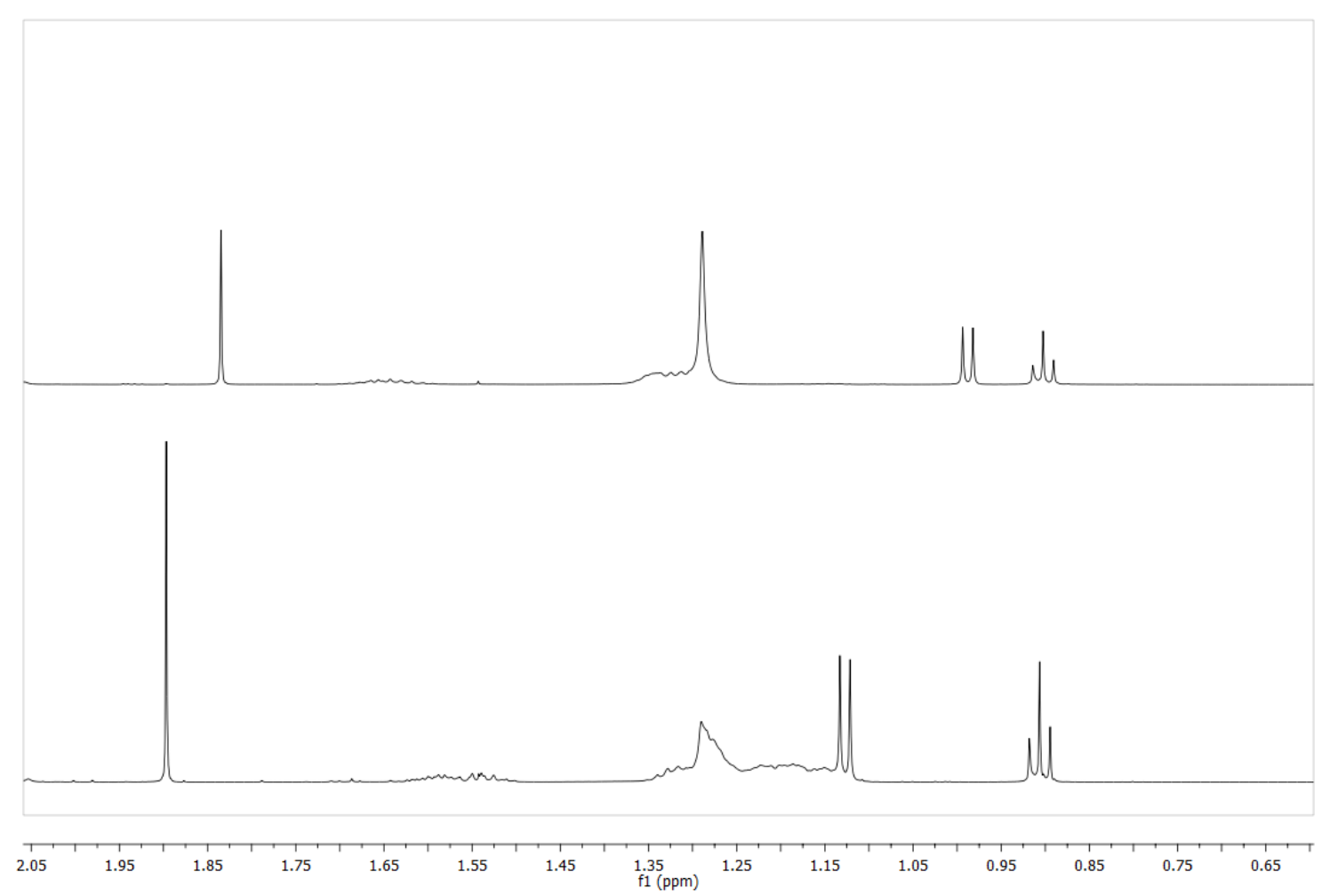

Figure 6.15 Aliphatic region, ${ }^{1} \mathrm{H}$ NMR spectra for the $\boldsymbol{R}$-ester (160, top panel) and $S$-ester (161, bottom panel) in $\mathrm{CD}_{3} \mathrm{OD}(600 \mathrm{MHz})$.

To futher confirm these results, ${ }^{19} \mathrm{~F}$ NMR analysis of the esters was performed as originally proposed by Mosher [309, 310]. In this method it was proposed that if the phenyl ring was on the same side as the most bulky substituent of the ester, then coplanarity is lost resulting in a shift of the $\mathrm{CF}_{3}$ from the desheilding zone to the sheilding zone of the carbonyl (Figure 6.16).

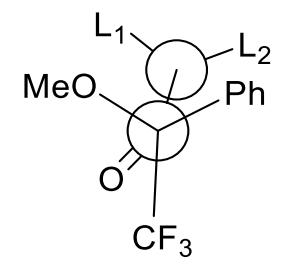

R-MTPA ester

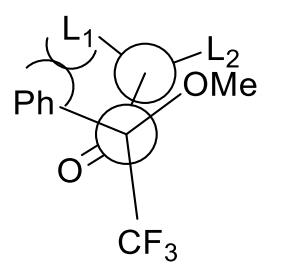

S-MTPA ester
$\mathrm{L}_{1}$ : bulky substituent

$\mathrm{L}_{2}$ : non-bulky substituent

Figure 6.16 Steric effects influencing ${ }^{19}$ F NMR shifts

In this figure the phenyl ring of $S$-MTPA ester is on the same side as the most bulky substituent. This results in the loss of coplanarity and the resulting shift in sterics moves $\mathrm{CF}_{3}$ to the sheilding zone of the carbonyl. 
Based on the ${ }^{1} \mathrm{H}$ NMR shifts observed from the $R$ and $S$ - MTPA esters (Figure 6.13), it is expected that for the $R$-ester the phenyl ring is on the same side as the $N$-acyl group. If this holds true, the steric interaction of the phenyl ring and the bulky $N$-acyl group would cause the $\mathrm{CF}_{3}$ to resonate further up-field [311]. Consequently, the resulting shift in the $S$-ester would be at higher ppm, and this is exactly what was observed (Figure 6.17).

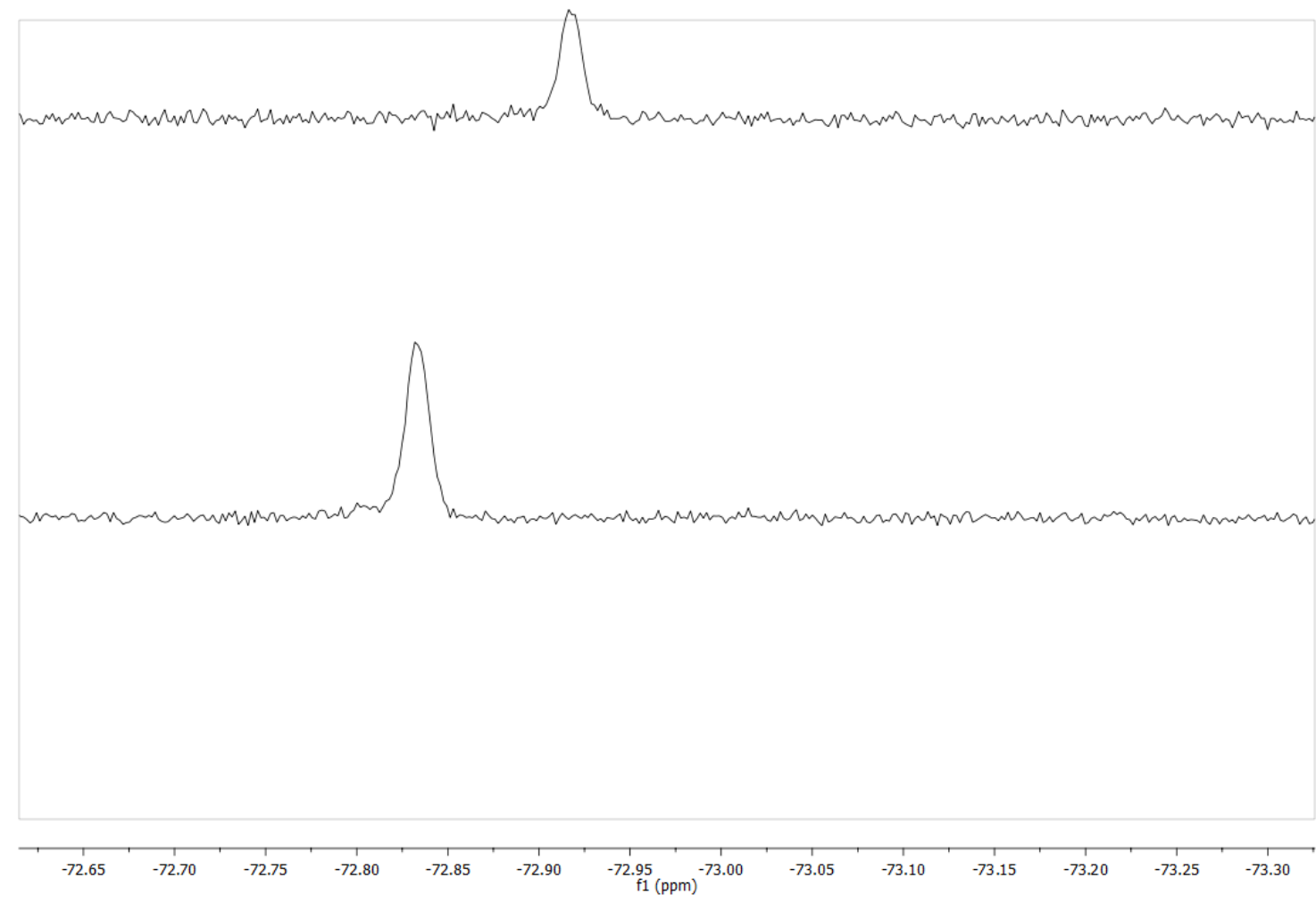

Figure 6.17 ${ }^{19} \mathrm{~F}$ NMR shifts $(280 \mathrm{MHz})$ in $\mathrm{CD}_{3} \mathrm{OD}$ for the $R$-ester (160, top panel) and $S$-ester (161, bottom panel) in $\mathrm{CD}_{3} \mathrm{OD}$. 
Table $6.2{ }^{1} \mathrm{H}(600 \mathrm{MHz}),{ }^{13} \mathrm{C}(150 \mathrm{MHz})$ NMR data of 3-epi-xestoaminol-C (156) $\left(\mathrm{CD}_{3} \mathrm{OD}\right)$.

\begin{tabular}{ccccc}
\hline & $\delta_{\mathrm{C}}$ & $\delta_{\mathrm{H}}(J$ in Hz$)$ & $\mathrm{COSY}$ & HMBC \\
\hline 1 & $16.1, \mathrm{CH}_{3}$ & $1.27(\mathrm{~d}, 6.7 \mathrm{~Hz})$ & 2 & $2,3,7$ \\
2 & $53.5, \mathrm{CH}$ & $3.09($ quin, $6.8 \mathrm{~Hz})$ & 1,3 & $1,3,4$ \\
3 & $73.2, \mathrm{CH}$ & $3.44(\mathrm{ddd}, 8.5,7.0,3.0 \mathrm{~Hz})$ & $2,4 \mathrm{a}, \mathrm{b}$, & $1,4,5$ \\
$4 \mathrm{a}$ & $34.7, \mathrm{CH}_{2}$ & $1.40, \mathrm{~m}$ & $3,4 \mathrm{~b}$ & 3,5 \\
$4 \mathrm{~b}$ & & $1.56, \mathrm{~m}$ & $3,4 \mathrm{a}$ & 5,9 \\
$5 \mathrm{a}$ & $26.4, \mathrm{CH}_{2}$ & $1.39, \mathrm{~m}$ & & 2,3 \\
$5 \mathrm{~b}$ & & $1.54, \mathrm{~m}$ & & 6 \\
$6-11$ & $30.5-30.8, \mathrm{CH}_{2}$ & $1.30, \mathrm{~m}$ & & \\
12 & $33.1, \mathrm{CH}_{2}$ & $1.29, \mathrm{~m}$ & 14 & 13 \\
13 & $23.8, \mathrm{CH}_{2}$ & $1.31, \mathrm{~m}$ & 14 & 12,14 \\
14 & $14.5, \mathrm{CH}_{3}$ & $0.91(\mathrm{t}, 7.0 \mathrm{~Hz})$ & 12,13 & 12,13 \\
\hline
\end{tabular}

\subsubsection{Compounds from the genus Xiphophora}

Algae belonging to the genus Xiphophora have not been extensively studied in the field of marine natural products chemistry. The majority of studies on this genus are marine ecological studies focusing on topics such as ammonia, urea and nitrate uptake, or phylogenetics as well as surveys of the species of algae from different intertidal zones. However, there has been one reported study that involved the isolation of a Penicillium sp. fungus from Xiphophora gladiata, which led to the isolation of two 2pyridones (162 and 163) [312].<smiles>C[C@H]1C[C@H](C)[C@@H]2[C@@H](C)Oc3cc[nH]c(=O)c3[C@@]2(C)C1</smiles>

162<smiles>C[C@H]1C[C@H](C)C[C@H](c2c(O)cc[nH]c2=O)[C@@H](C)[C@@]1(C)O</smiles>

163

The isolation of $\mathbf{1 6 2}$ and $\mathbf{1 6 3}$ was a result of the research group's focus on compounds isolated from fungi, and therefore the metabolites of the algae were not investigated. As X. gladiata belongs to the same genus as X. chondrophylla, it is 
possible that amino alcohols may also be present in $X$. gladiata, however this remains to be investigated.

The amino alcohols xestoaminol A-C (107-109) were first reported by Jiménez and Crews as stated earlier. To elucidate their configurations, xestoaminol A was derivatised to form an oxazolidinone, the coupling constant between $\mathrm{H}-2$ and $\mathrm{H}-3$ was approximately $7.5 \mathrm{~Hz}$, suggestive of a cis relationship between the protons of the five membered ring system. Based on these results, Jiménez and Crews proposed the $2 S^{*}, 3 R^{*}$ stereochemistry for xestoaminol A. The proposed $2 S^{*}, 3 R^{*}$ stereochemistry for xestoaminol C (109) was based on similarities of the C-2 and C3 carbons chemical shifts of xestoaminol C to xestoaminol A [284]. As stated earlier in section 6.4.2, the relative stereochemistry of 3-epi-xestoaminol C (156) was also elucidated by generating the oxazolidinone derivative and the coupling constant measured was $6.5 \mathrm{~Hz}$, which indicated a trans relationship between H-2 and H-3 in 157 [274]. Sata et al. have previously used differences in coupling constant to elucidate the relative configuration of two epimers, amaminols A (142) and B (143) which differ at the 2-position [274]. It should be noted that the absolute configuration of $\mathbf{1 0 9}$ was elucidated as $2 S, 3 R$ for the two stereogenic centers, 11 years later using Mosher's method [278].

The research described in this thesis applied established and accepted methods to elucidate the relative and absolute configurations of 3-epi-xestoaminol C. Sata et al. as well as Jiménez and Crews formed the oxazolidinone derivative of their respective amino alcohols and then measured the coupling constants between the oxymethine and aminomethines. Sata et al. measured the differences between two different epimers and showed that a coupling constant of $6.5 \mathrm{~Hz}$ indicated a trans relationship and $7.3 \mathrm{~Hz}$ indicated a cis relationship within the oxazolidinone ring [274]. This translates to the opposite when considering the respective linear forms, i.e. a cis relationship for the oxazolidinone indicates a trans relationship for the underivatised form.

Garrido et al. applied Mosher's method for elucidating the absolute stereochemistry xestoaminol $\mathrm{C}$ by [278]. With a few minor differences the same approach was applied to elucidate the absolute configuration of 156. Garrido et al. identified an $R$ configuration for the $\mathrm{C}-3$ center, thus elucidating the absolute configuration of 
xestoaminol $\mathrm{C}$ as $2 S, 3 R$ [278]. Using the methods detailed, the compound isolated from X. chondrophylla was identified as the C-3 epimer of xestoaminol C.

Amino alcohols or 1-deoxysphingoids, tend to be isolated from sponges and ascidians, which makes this the first report of the isolation of this class of compounds from algae. The closest reports from marine algae involve the isolation of sphingosine derivatives from the red alga Laurencia nidifica and the green alga Ulva fasciata [313, 314]. This research has therefore identified the presence of sphingoids from brown algae for the first time.

\subsubsection{Bioactivity}

The crude extract of $X$. chondrophylla had an $\mathrm{MIC}_{90}$ value of $200 \mu \mathrm{g} / \mathrm{mL}$, and after purification the pure compound had an $\mathrm{MIC}_{90}$ of $15 \mu \mathrm{g} / \mathrm{mL}(65.1 \mu \mathrm{M})$, an overall 13fold improvement in activity. This activity is consistent in all species of bacteria studied (Figure 6.1), except Pseudomonas aeruginosa which was resistant to the highest concentration tested $(60 \mu \mathrm{g} / \mathrm{mL})$. In addition to the growth inhibition assay, the compound was tested against stationary phase M. tuberculosis H37Ra, yielding an $\mathrm{MIC}_{90}$ of $65.1 \mu \mathrm{M}$ in this assay as well (Figure 6.2). This hints to the possibility of a generic mechanism of action, possibly a detergent-like activity, which is not unexpected when considering the structure of the compound, the polar head and nonpolar tail, which make for an ideal membrane disruptor.

When the compound was investigated for its activity against mammalian cell lines the results proved more interesting. Comparison of the HL-60 ( $\mathrm{IC}_{50} 8.8 \mu \mathrm{M}$ ) to the HEK $\left(\mathrm{IC}_{50} 18.0 \mu \mathrm{M}\right)$ data proves a slight specificity of the compound towards the HL-60 cancer cell line compared to the HEK cell line, which was used as a control to measure general toxicity (Figure 6.3). Though the therapeutic index of this compound is 2 , which is very low and far from ideal, this may still warrant future studies to investigate this compound. Furthermore, the availability of $\mathbf{1 5 6}$ in such a high mass as well as the observed activity against $S$. cerevisiae allowed profiling of its mechanism of action using yeast chemical genetics, which may shed light on the current understanding of how 1-deoxysphingoids cause their observed activity.

When studying the effects the compound has on 1A9 cells transitioning from G2 to M-phase, 3-epi-xestoaminol C (156) does not inhibit this stage of the cell cycle (this 
work was done with the help of Dr. Arun Kanakkanthara, Appendix A, page 173). The mechanism of action to induce cell death could be via alternative ways mentioned earlier for ES-285, such as disruption of actin cell fibres causing the destabilization of the cytoskeleton of the cell [290], activation of caspase 3 and 12, the phosphorylation of p53 and activation of isoforms of protein kinase $\mathrm{C}$ (PKC), inducing atypical cell death [291], or alternatively by causing ceramide induced apoptosis via activation of PKC $\zeta$ [292-295].

\subsubsection{SAR study}

Table 6.1 displays the results of the basic SAR work done on 3-epi-xestoaminol C. Testing oxazolidinone derivative 157 against $M$. tuberculosis H37Ra resulted in a drop in the $\mathrm{MIC}_{90}$ to $60 \mu \mathrm{g} / \mathrm{mL}$. This suggests that the polar head is crucial for bioactivity. The diacetyl and $N$-acetyl derivatives generated for carrying out Mosher's analysis were also tested for activity. This indicated that modification to both the $\mathrm{NH}$ and $\mathrm{OH}$ resulted in a loss of activity with a maximum of $80 \%$ inhibition observed at the highest tested concentration of $60 \mu \mathrm{g} / \mathrm{mL}$. Solely modifying the NH via the addition of an acetyl group also resulted in complete loss of activity. A clear trend is therefore noted, where any modifications to the polar head group causes a decrease in activity. This loss of activity is further highlighted by the bioactivity results of the Mosher's esters with both showing a lack of activity at the highest concentration tested $(60 \mu \mathrm{g} / \mathrm{mL})$ (Table 6.1).

\subsubsection{Mechanism of action}

In order to elucidate the mechanism of action of this compound in mycobacteria, 3epi-xestoaminol $\mathrm{C}$ was screened against a library of random transposon mutants. The top 120 mutants were selected and testing them for sensitivity followed by validation of 31 mutants using dose response curves did not identify any mutants that were more sensitive to the compound. This may indicate that either this compound targets an essential gene, which would explain why no specific mutant was identified, or alternatively that this compound acts as a detergent, which could also explain its activity against a range of microbes.

In an alternative approach, the generation of resistant mutants was attempted. This involved the generation of random transposon mutants that were grown on plates at a 
range of concentrations for over four weeks. However, growth was only observed at the non-inhibitory concentration, with no growth of mutants observed at concentrations above $7.5 \mu \mathrm{g} / \mathrm{mL}$.

\subsubsection{Chemical genetics}

To gain further understanding of how the compound interacts in a eukaryotic system, the powerful tool of yeast genetics was used to identify pathways that this compound interacts with to generate the observed biological effect. A synthetic genetic array (SGA) was performed in a liquid assay system optimised to allow for minimal use of drug. The chemical genetics results described in this section were obtained by Nazmi H. Fadzilah.

Chemical genetics employs a library containing 4800 non-essential gene deletion mutants of Saccharomyces cerevisiae (baker's yeast).[315] The library was screened at the $\mathrm{IC}_{20}$ concentration as this ensures for bioactivity as well as allowing for the identification of mutants that are the most sensitive to drug treatment. A dose response assay in $S$. cerevisiae identified an $\mathrm{IC}_{50}$ of $17.1 \mu \mathrm{M}$, and an $\mathrm{IC}_{20}$ of $3.5 \mu \mathrm{M}$. The library of non-essential mutants was grown for $16 \mathrm{~h}$ in the presence of 3-epixestoaminol $\mathrm{C}$ at the $\mathrm{IC}_{20}$ concentration. Hits were selected using a $t$ test with a Bonferroni correction adjusting the $p$ value to 0.017 . Using this selection criterion, sensitive strains which displayed less than $70 \%$ residual growth and resistant strains that had greater than $130 \%$ growth in comparison to DMSO controls were chosen for validation. This identified 805 hits, using the same selection criterion validation of the 805 hits had identified 28 mutants of significance (two of which were of dubious annotation and therefore excluded).

This resulted in 12 networks representing 19 genes involved in known pathways and one network with seven genes as part of unknown pathways (Figure 6.4). The largest number are genes were involved in unknown processes, followed by genes involved in lipid metabolism (AIM6, PLB3, CRC1, SPS19 and OPI1), and actin cytoskeleton organization (AKL1, RHO2 and SLA1) (Figure 6.4).

As sphingolipids are believed to regulate lipid metabolism, it is expected that this process should be affected by 3-epi-xestoaminol C [316]. Another process that was expected to appear in our network map was actin cytoskeleton organization, as it has 
been previously suggested that ES-285 prevents the formation of actin stress fibres by decreasing the activity of Rho proteins [290]. Our results correlate with the possible mechanisms of action reported for ES-285.

Furthermore, as this type of study has not been performed before for 1-deoxysphingoids, it sheds further light on other possible pathways that may be involved in the mechanism of action of 1-deoxysphingoids. These new pathways include RNA catabolism, protein targeting and phosphorylation, amongst others (Figure 6.4). Studying how 1-deoxysphingoids interact with these new and unknown pathways may allow for a better understanding of the mechanism of action of these compounds. In addition, 156 may also help in identifying what role these unknown pathways play within cells. Interestingly, mutants in the ceramide synthase pathways did not appear in this screen, this may be due to the statistical cut off used to identify hits. It would therefore be interesting to pick individual mutants involved in ceramide synthesis and study the phenotypic effects when they are exposed to 3-epixestoaminol C (156).

\subsubsection{Future directions}

As the HPLC attached to an ELSD was available for use near the end of this study, a repeat isolation assisted by the ELSD may help in purifying 3-epi-xestoaminol $\mathrm{C}$ in fewer and less laborious steps. As the 1-deoxysphingoids have been linked to alteration of ceramide levels as was the case for ES-285, it would be interesting to study the effect of $\mathbf{1 5 6}$ on mutants involved in this pathway. Studying the effects of compounds with the same configuration, however with varying levels of saturation would also be of interest. 


\section{Chapter 7 Concluding remarks}


Marine natural products research has led to the isolation of a plethora of novel compounds. The recent improvement in culturing methods of microbes once believed to be unculturable as well as genome mining methods is sure to lead to the identification of more unique compounds. Dereplication issues are a common problem in this field, the isolation of known compounds is a frustrating waste of time and resources that can be overcome using unique isolation methods. However, when pursuing bioactivity, the goal is often to isolate the bioactive, which may be a known compound.

The research described in this thesis employed a classic approach of bioassay-guided fractionation using whole cell assays. Whole cell assays are better suited for screening of crude extract as the complex nature of crude extracts can often interfere with more sensitive targeted biochemical assays.

The NIWA and VUW MNP libraries were screened to select extracts with potentially new anti-tubercular compounds. These screens led to the identification of a number of hits of which five were chosen due to availability, activity, and amount.

Due to time constraints as well as loss of activity, eventually three samples were pursued. As stated before, a common issue encountered is re-isolation of known compounds, which was observed with one sample that resulted in the isolation of common fatty acids from the active extract of blob fish liver.

Another common issue often encountered is low sample mass for the isolated compound. The fractionation of the Latrunculia sponge led to isolation of a novel compound, however the compound responsible for anti-mycobacterial activity was unable to be purified and identified. This compound was present at such low levels that it was impossible to elucidate any structural information. Furthermore, even rimarikiamide A (98) was not isolated in sufficient amounts to allow for the elucidation of relative and absolute configuration. The next step will be to synthesize two of the four possible stereoisomers and compare optical rotation and NMR data to the natural product to confirm the absolute configuration of the natural product, as well as further investigate its bioactivity. 
The isolation work performed on the brown algae Xiphophora chondrophylla led to the isolation of a new compound, 3-epi-xestoaminol C (156) this compound was equally active against all strains of bacteria except Pseudomonas aeruginosa.

Xestoaminol C (109) was first isolated from a Xestospongia sp. sponge. The spectral data reported by Jiménez and Crews correlated very closely to the data of $\mathbf{1 5 6}$, with the exception of a signature methyl doublet and oxymethine. The relative stereochemistry of the amino alcohol was identified by locking the compound's amine and alcohol moieties in an oxazolidinone ring followed by measuring the coupling constant between the amino- and oxymethine, as well as from 1D NOE experiments. Generating the Mosher's esters allowed characterisation of the absolute configuration of the compound; this validated the fact that this compound has a different configuration from 109, and is therefore a new compound. The bioassay data generated from this work and crude SAR analysis identified the polar head as crucial for activity.

This compound proved to be a better cytotoxic agent towards HL-60 cells. Therefore the next logical step was to study the effects of this compound on eukaryotic cells. A model organism that allows for genome wide analysis at VUW was the S. cerevisiae gene deletion set. This was employed to perform SGA studies which led to the identification of 13 categories that represented pathways 3-epi-xestoaminol $\mathrm{C}$ interacts with, two of which were expected, one represented unknown pathways and the others were novel. This study therefore highlights cellular processes yet to be implicated in the mechanism of action of 1-deoxysphingoids, which will require further studies on the mutants identified. As 1-deoxysphingoids have also been shown to affect ceramide levels within cells, studying how mutants involved in this pathway interact with 3-epi-xestoaminol $\mathrm{C}$ may also be of value to gain a better understanding of these compounds.

The $X$. chondrophylla project saw the progress of a sample from the crude stages through to a pure compound, following which derivatives were made to elucidate the absolute stereochemistry, which aided in performing SAR studies. Finally, the SGA work in S. cerevisiae ensured the overarching three goals of my thesis were achieved. 
It is interesting to note that all the compounds isolated through this work share similar features. These compounds all have polar head groups and long hydrophobic chains, which may be due to the assay used in this approach.

Looking at the activities of palmitic acid and 3-epi-xestoaminol $\mathrm{C}$ against M. tuberculosis H37Ra, it is apparent that a polar head group is important for compounds such as these. Although the exact reason for this was not investigated, the presence of a polar head as well as a non-polar tail may allow for better solubility of the compound, which would allow for more of it to be in solution and would aid in traversing both hydrophilic and hydrophobic environs. Furthermore the amphiphilic nature of the compound may allow for better permeation through the bacterial cell wall or alternatively cause lysis of cells due to detergent type activity.

When looking at chain length it seems that C14-16 carbon chains may be effective as observed with the activity of 3-epi-xestoaminol $\mathrm{C}$ and palmitic acid. It would be interesting to study the effects of other $\mathrm{C} 14$ fatty acids to better understand the effects of chain lengths. When looking at level of unsaturation it can be observed that having saturated chains is more favoured.

If this project were to be redesigned and performed again, a better possible approach could include performing the library screens directly against M. tuberculosis H37Ra. Due to the increased bioactivity observed in the crude extract of Latrunculia sp. sponge against M. tuberculosis H37Ra, the implied specificity may hint at the possibility of other specific hits being overlooked. In addition, a counter screen could be introduced by screening the extract against HEK cells. Although crude extracts may have higher toxicity due to the possibility of higher levels of surfactants present, it may allow for a better selection of hits to be made. 
Appendices 


\section{Appendix A:}

\section{NIWA library screening raw data}

Table 7.1 Raw data of NIWA library screen vs. $M$. smegmatis

Plates 12, 13 and 14 were kindly donated by NIWA and screened for antimycobacterial activity. Top hits highlighted in yellow, positive control in red and negative control in green. Wells are represented as either P12, 13 or 14 followed by the well number for plates 12, 13 and 14 respectively. Standard error (std error) values are also listed for each sample.

\begin{tabular}{lrrrr}
\hline Well number & \multicolumn{1}{l}{$\begin{array}{l}\text { GFP } \\
\text { average }\end{array}$} & \multicolumn{1}{l}{$\begin{array}{l}\text { GFP } \\
\text { std error }\end{array}$} & $\begin{array}{l}\text { Resazurin } \\
\text { average }\end{array}$ & \multicolumn{1}{l}{$\begin{array}{l}\text { Resazurin } \\
\text { std error }\end{array}$} \\
\hline P12C11 & 95.29507 & 0.785983 & 86.38034 & 3.157086 \\
P12E9 & 95.23894 & 0.81616 & 86.08682 & 2.949949 \\
Positive & 94.17888 & 0.805538 & 86.0707 & 1.28972 \\
\hline P12D2 & 95.44557 & 0.774508 & 85.98647 & 2.898718 \\
P12B11 & 95.22699 & 0.795667 & 85.63803 & 3.24214 \\
P12H5 & 95.29462 & 0.798398 & 84.61087 & 2.82355 \\
P12A5 & 95.18794 & 0.741449 & 84.50358 & 2.231667 \\
P12H8 & 94.91964 & 0.883313 & 84.38737 & 3.549344 \\
P12F4 & 94.65777 & 0.900614 & 83.94367 & 3.177821 \\
P14D8 & 79.84463 & 10.39383 & 79.52913 & 6.688682 \\
P12C5 & 92.33131 & 3.153455 & 74.69969 & 9.574227 \\
P14A10 & 76.53547 & 13.60222 & 74.00939 & 12.16117 \\
P12H2 & 65.38177 & 30.07546 & 72.72987 & 10.19009 \\
P14C8 & 74.31914 & 15.79287 & 72.54576 & 14.13008 \\
P14F6 & 85.78419 & 4.406677 & 71.18628 & 16.53152 \\
P12E7 & 80.54696 & 14.88215 & 70.23149 & 13.03872 \\
P12G7 & 88.10912 & 7.205349 & 70.19471 & 12.21514 \\
P13C4 & 86.06836 & 9.197341 & 69.88816 & 17.19673 \\
P12G11 & 91.05706 & 4.254647 & 67.37275 & 14.86076 \\
P12B4 & 82.64904 & 12.74172 & 66.18606 & 17.17667 \\
P12D11 & 49.1333 & 45.78911 & 66.1028 & 11.47069 \\
P12B10 & 79.7254 & 15.5422 & 64.57238 & 15.22279 \\
P12E1 & 73.53752 & 21.89476 & 63.24637 & 20.81483 \\
P14G1 & 87.61734 & 4.389685 & 62.15067 & 8.500751 \\
P14G4 & 87.94448 & 1.672556 & 60.93931 & 19.99167 \\
P13C3 & 88.19733 & 7.070043 & 60.48392 & 25.30041 \\
P13F3 & 80.15213 & 15.23033 & 59.29562 & 27.22728 \\
P14H3 & 89.10644 & 1.622557 & 59.17182 & 26.97998 \\
P14B1 & 51.06214 & 40.52924 & 47.83945 & 23.17653 \\
P13F2 & 61.09333 & 33.45879 & 45.45379 & 24.96453 \\
P12D4 & 57.49756 & 32.63582 & 39.50918 & 33.59945 \\
P12D6 & 63.57766 & 28.34269 & 38.3046 & 35.8992 \\
\hline & & & & \\
\hline
\end{tabular}




\begin{tabular}{|c|c|c|c|c|}
\hline P14A7 & 70.86429 & 9.036251 & 36.84229 & 27.03343 \\
\hline P13G3 & 69.56712 & 24.46582 & 35.55058 & 31.69946 \\
\hline P14C9 & 49.30522 & 23.41664 & 33.04114 & 28.47672 \\
\hline P12D5 & 68.03184 & 21.56482 & 32.43605 & 35.22354 \\
\hline P14D11 & -15.7199 & 42.80098 & 29.93673 & 2.796792 \\
\hline P12C6 & 70.14362 & 12.3702 & 28.00977 & 34.27149 \\
\hline P12A9 & 82.31134 & 8.054148 & 26.9769 & 33.4664 \\
\hline P13B2 & 71.13187 & 23.20145 & 26.87892 & 34.10814 \\
\hline P14C7 & 53.16032 & 12.48884 & 25.55587 & 8.495419 \\
\hline P14G5 & 63.19724 & 11.91114 & 25.42668 & 22.88006 \\
\hline P14B8 & 47.98046 & 16.84785 & 25.13695 & 7.053966 \\
\hline P14E7 & 49.87826 & 15.84849 & 25.01259 & 1.765235 \\
\hline P14E8 & 3.435204 & 12.42514 & 24.75312 & 11.46179 \\
\hline P14D6 & 68.49973 & 11.54012 & 24.21785 & 1.913252 \\
\hline P14E6 & 53.46062 & 23.30951 & 23.97877 & 5.462849 \\
\hline P14B7 & 47.95174 & 13.52469 & 23.912 & 16.88196 \\
\hline P14G10 & 31.32469 & 21.25331 & 22.74761 & 3.867035 \\
\hline P14G7 & 36.47978 & 32.63412 & 21.95137 & 1.840131 \\
\hline P14E4 & 51.67138 & 20.94187 & 21.71609 & 2.734935 \\
\hline P14E11 & 19.13935 & 24.94786 & 21.40771 & 6.166837 \\
\hline P14F10 & -8.16547 & 48.91745 & 21.11393 & 6.880446 \\
\hline P14C6 & 50.46009 & 22.35922 & 21.05689 & 3.309809 \\
\hline P14E9 & 44.116 & 16.96219 & 20.98442 & 11.91788 \\
\hline P13B5 & 46.76197 & 48.55091 & 20.52903 & 65.74664 \\
\hline P14E5 & -10.3081 & 33.59287 & 20.29615 & 8.892144 \\
\hline P14F8 & 27.96623 & 26.44523 & 19.7249 & 4.788833 \\
\hline P14F4 & 21.3651 & 36.61031 & 19.7137 & 6.165707 \\
\hline P14G8 & 27.28424 & 34.13498 & 19.39925 & 2.782101 \\
\hline P14B2 & 60.67015 & 19.44923 & 19.18749 & 4.301774 \\
\hline P14C2 & 64.68015 & 13.99315 & 18.60105 & 6.759065 \\
\hline P12H9 & 75.27249 & 13.65901 & 18.58306 & 38.66039 \\
\hline P14C5 & 60.94756 & 9.250122 & 17.6841 & 2.371108 \\
\hline P14G6 & -2.55169 & 48.03772 & 17.61007 & 6.378801 \\
\hline P14G2 & 14.21108 & 34.27734 & 17.41164 & 15.3916 \\
\hline P14A8 & 33.91814 & 17.58041 & 17.33406 & 3.371426 \\
\hline P14F5 & 37.62318 & 36.35582 & 17.25284 & 4.997432 \\
\hline P14G3 & 27.32602 & 35.76232 & 17.24924 & 3.812852 \\
\hline P14F11 & 28.84561 & 29.12361 & 17.21426 & 5.242239 \\
\hline P14B5 & 56.666 & 10.1577 & 17.19392 & 6.036702 \\
\hline P12F5 & 69.06763 & 11.45544 & 17.10833 & 30.20712 \\
\hline P12D10 & 70.23466 & 14.68935 & 17.00887 & 37.72978 \\
\hline P13F10 & 56.6338 & 14.76691 & 16.75626 & 20.25039 \\
\hline P14C10 & 56.85615 & 7.524538 & 16.68879 & 1.451631 \\
\hline $\mathrm{P} 14 \mathrm{C} 12$ & 69.56004 & 2.807874 & 16.60262 & 18.89024 \\
\hline P13D5 & 69.32286 & 11.68947 & 16.27412 & 16.58991 \\
\hline P14D5 & 72.3954 & 1.938687 & 15.82492 & 9.756835 \\
\hline
\end{tabular}




\begin{tabular}{|c|c|c|c|c|}
\hline P12G12 & 29.23471 & 35.89494 & 15.61437 & 4.148292 \\
\hline P14C4 & 45.91544 & 28.8453 & 15.39723 & 5.21136 \\
\hline P14E1 & 15.81121 & 23.72353 & 15.35797 & 11.95728 \\
\hline P14H9 & 37.37998 & 27.72498 & 15.33101 & 4.833102 \\
\hline P13E5 & 74.45022 & 2.457501 & 15.32738 & 18.02214 \\
\hline P14F3 & 22.31453 & 27.11177 & 15.21476 & 6.446794 \\
\hline P13E10 & 57.1752 & 22.91158 & 14.78389 & 15.13841 \\
\hline P13B7 & 60.70775 & 9.274539 & 14.6302 & 23.13163 \\
\hline P13D8 & 69.07614 & 9.93776 & 14.34612 & 20.50569 \\
\hline P14E2 & 39.19593 & 26.29932 & 14.34283 & 7.568437 \\
\hline P14C1 & 18.0416 & 24.64411 & 13.96777 & 14.86596 \\
\hline P13C5 & 78.82951 & 4.784819 & 13.96105 & 19.45644 \\
\hline P13G5 & 69.64096 & 20.74357 & 13.85236 & 38.74775 \\
\hline P14C3 & 41.04125 & 26.34051 & 13.28868 & 8.387458 \\
\hline P13B1 & 44.52683 & 43.27951 & 13.23391 & 21.78682 \\
\hline P14D2 & 53.9266 & 12.1028 & 13.13169 & 6.563468 \\
\hline P14B12 & 39.33416 & 28.3577 & 13.02601 & 11.31142 \\
\hline P13G4 & 82.65762 & 4.760891 & 12.9056 & 16.7919 \\
\hline P14D3 & 37.14536 & 25.56468 & 12.75301 & 5.807382 \\
\hline P13E6 & 74.49791 & 5.151318 & 12.69926 & 21.36707 \\
\hline P14H8 & 41.57048 & 23.14885 & 12.29052 & 4.769722 \\
\hline P14B9 & 29.04049 & 6.906667 & 12.2193 & 10.47905 \\
\hline P13C7 & 78.14485 & 7.030314 & 12.09265 & 20.67555 \\
\hline P12D8 & 31.1107 & 39.33485 & 11.93888 & 28.49429 \\
\hline P13C8 & 64.07387 & 10.25899 & 11.73318 & 24.61139 \\
\hline P13F7 & 62.11512 & 9.044507 & 11.68964 & 14.1738 \\
\hline P14F9 & 15.18114 & 27.34339 & 11.55868 & 2.146023 \\
\hline P14D7 & 61.08407 & 2.7064 & 11.54734 & 19.75837 \\
\hline P14H12 & 47.13213 & 14.99895 & 11.54464 & 14.61622 \\
\hline P13A8 & 61.05464 & 7.360603 & 11.5295 & 22.66638 \\
\hline P14B3 & 32.47928 & 18.86657 & 11.50742 & 1.90807 \\
\hline P14H6 & 21.26094 & 27.86608 & 10.82551 & 4.592332 \\
\hline P13A4 & 66.99853 & 11.48356 & 10.52092 & 22.08381 \\
\hline P13E3 & 78.61078 & 4.938423 & 10.43951 & 22.96065 \\
\hline P13G6 & 52.02614 & 6.498841 & 9.989098 & 20.71243 \\
\hline P13B11 & 54.63023 & 16.13409 & 9.873841 & 21.80595 \\
\hline P14D12 & 28.13839 & 20.54754 & 9.866753 & 15.01883 \\
\hline P13B3 & 57.62392 & 12.70165 & 9.59344 & 21.62048 \\
\hline P14E12 & 31.85651 & 18.16397 & 9.459336 & 5.571199 \\
\hline $\mathrm{P} 12 \mathrm{~F} 2$ & 16.11191 & 16.22787 & 9.288915 & 14.8254 \\
\hline P13G8 & 64.9466 & 6.885534 & 9.151431 & 23.37667 \\
\hline P13D2 & 65.69508 & 7.682957 & 9.033771 & 21.50588 \\
\hline P14H5 & 8.680589 & 33.75608 & 8.947887 & 3.791927 \\
\hline P13D10 & 59.33892 & 19.75762 & 8.860935 & 19.83009 \\
\hline P14B10 & 42.78492 & 17.36829 & 8.721749 & 8.617814 \\
\hline P13D9 & 67.43655 & 11.37215 & 8.599439 & 26.26022 \\
\hline
\end{tabular}




\begin{tabular}{|c|c|c|c|c|}
\hline P13H6 & 64.21682 & 11.16812 & 8.413744 & 21.63533 \\
\hline P12A8 & 64.69136 & 10.25722 & 8.397333 & 20.10236 \\
\hline P12C7 & 62.57825 & 10.58206 & 8.321585 & 30.69887 \\
\hline P13C10 & 67.70048 & 9.27671 & 8.225073 & 23.17331 \\
\hline P12A11 & 54.71581 & 9.358774 & 8.057547 & 20.18418 \\
\hline P14H1 & 53.34001 & 4.460681 & 8.033757 & 21.58946 \\
\hline P13H11 & 75.21667 & 10.63116 & 7.962502 & 43.87446 \\
\hline P14A2 & 7.278837 & 13.2975 & 7.830901 & 9.951199 \\
\hline P13F8 & 50.79972 & 10.11439 & 7.669997 & 19.27028 \\
\hline P14H4 & 39.53698 & 10.33974 & 7.567364 & 6.568749 \\
\hline P13F5 & 63.48538 & 8.849496 & 7.222446 & 17.27257 \\
\hline P13H7 & 53.19039 & 16.20456 & 7.126441 & 17.85725 \\
\hline P13H5 & 61.16707 & 3.049284 & 7.115328 & 18.58506 \\
\hline P14D1 & 38.08819 & 34.61624 & 6.814165 & 13.30402 \\
\hline P13F9 & 55.4294 & 5.015927 & 6.763839 & 21.76425 \\
\hline P13G10 & 55.28635 & 14.76988 & 6.726579 & 15.25053 \\
\hline P12C3 & 48.60107 & 8.431397 & 6.590149 & 5.760599 \\
\hline P13B10 & 57.43152 & 11.83799 & 6.363667 & 21.5196 \\
\hline P13D6 & 65.51006 & 19.01638 & 6.350273 & 21.98976 \\
\hline P12E2 & 71.85173 & 4.66917 & 6.344223 & 24.93408 \\
\hline P12D3 & 71.1574 & 8.609529 & 6.30302 & 28.15031 \\
\hline P13C9 & 69.56654 & 10.48855 & 6.297048 & 16.53759 \\
\hline P12C10 & 68.00201 & 9.616971 & 6.231508 & 19.17401 \\
\hline P13E11 & 76.31375 & 3.70551 & 6.045135 & 23.55996 \\
\hline P12E5 & 68.61907 & 6.330934 & 5.557262 & 21.98866 \\
\hline P12A6 & 57.71936 & 1.301973 & 5.064177 & 17.58 \\
\hline P14C11 & 10.60718 & 21.81412 & 4.982768 & 10.29458 \\
\hline P13E2 & 61.23323 & 16.43361 & 4.822049 & 22.9139 \\
\hline P14A5 & -16.4687 & 48.64646 & 4.746931 & 17.85379 \\
\hline P13D7 & 70.92203 & 6.410861 & 4.41082 & 17.24668 \\
\hline P13E7 & 61.03934 & 19.52656 & 4.409845 & 24.14269 \\
\hline P13B4 & 79.59598 & 4.024533 & 4.342918 & 25.83868 \\
\hline P12F3 & 44.8628 & 36.53872 & 4.22125 & 36.011 \\
\hline P13D1 & 24.00031 & 31.74321 & 4.153307 & 5.733589 \\
\hline P12E8 & 79.68616 & 1.521917 & 4.14307 & 26.70217 \\
\hline $\mathrm{P} 14 \mathrm{~F} 2$ & 14.52617 & 26.40959 & 4.131409 & 24.03614 \\
\hline P14F12 & 48.08661 & 7.866409 & 3.837718 & 16.40139 \\
\hline P13E12 & 23.79505 & 33.47606 & 3.778476 & 15.28476 \\
\hline P14B4 & 33.99386 & 13.52558 & 3.422502 & 14.01213 \\
\hline P14A11 & 11.06668 & 16.55673 & 3.297886 & 7.922972 \\
\hline P12B5 & 52.1512 & 38.33371 & 3.127009 & 41.4558 \\
\hline P12H10 & 81.29384 & 4.204137 & 2.823713 & 28.91615 \\
\hline P14A12 & 30.34584 & 19.23776 & 2.793087 & 6.21532 \\
\hline P12B6 & 54.66647 & 9.987044 & 2.664445 & 25.24767 \\
\hline $\mathrm{P} 14 \mathrm{H} 2$ & 13.92118 & 27.26741 & 2.573717 & 9.559425 \\
\hline P12F10 & 79.05507 & 1.653811 & 2.544383 & 24.63217 \\
\hline
\end{tabular}




\begin{tabular}{|c|c|c|c|c|}
\hline P13B6 & 59.98612 & 11.40872 & 2.488611 & 22.66604 \\
\hline P13H4 & 33.22263 & 11.54821 & 2.4225 & 16.63882 \\
\hline P13F1 & 40.5449 & 7.502826 & 2.131612 & 11.72879 \\
\hline P12F6 & 52.24805 & 15.40515 & 2.119675 & 11.85613 \\
\hline P13E8 & 35.6204 & 19.22529 & 2.092663 & 27.56439 \\
\hline P13F4 & 68.62508 & 5.490216 & 2.042568 & 22.91815 \\
\hline P12C4 & 63.50569 & 8.143879 & 1.884164 & 25.27153 \\
\hline P13A5 & 62.4339 & 3.883837 & 1.85076 & 41.17196 \\
\hline P13B9 & 59.88139 & 10.3023 & 1.752514 & 31.54771 \\
\hline P12A3 & 35.56098 & 7.305887 & 1.672668 & 13.25954 \\
\hline P12A10 & 52.13727 & 10.13055 & 1.435534 & 17.48855 \\
\hline P13H10 & 67.46127 & 6.511313 & 1.431417 & 16.00071 \\
\hline P13G11 & 41.55106 & 23.23634 & 1.134434 & 20.43297 \\
\hline P12D7 & 61.20281 & 16.86063 & 1.081377 & 26.45987 \\
\hline P13D3 & 80.97955 & 1.981237 & 1.068096 & 26.38719 \\
\hline P13C6 & 76.1625 & 3.490828 & 1.031579 & 24.7379 \\
\hline P12B8 & 51.07312 & 14.2143 & 0.986961 & 22.11304 \\
\hline P13C2 & 78.06332 & 4.960391 & 0.933395 & 25.76688 \\
\hline P13C11 & 53.87485 & 8.696173 & 0.789122 & 18.37219 \\
\hline P12D9 & 53.58877 & 6.771584 & 0.703301 & 23.69749 \\
\hline P13E9 & 57.01774 & 6.927957 & 0.675407 & 21.78598 \\
\hline P14H10 & 19.79333 & 29.65136 & 0.662297 & 7.325898 \\
\hline P14A1 & 52.57091 & 3.656583 & 0.616609 & 26.74211 \\
\hline $\mathrm{P} 12 \mathrm{H} 4$ & 34.7322 & 17.46375 & 0.574882 & 28.7213 \\
\hline $\mathrm{P} 12 \mathrm{C} 2$ & 30.27995 & 11.51482 & 0.393493 & 11.53775 \\
\hline P13H9 & 62.3895 & 2.493845 & 0.064536 & 15.2876 \\
\hline P13G9 & 62.55288 & 13.77852 & 0.001952 & 21.21672 \\
\hline P12E3 & 47.98199 & 23.28462 & -0.7108 & 16.20891 \\
\hline P12E11 & 73.93991 & 6.194231 & -0.9781 & 22.24723 \\
\hline P12H7 & 65.96385 & 12.26945 & -1.00993 & 23.9661 \\
\hline P12B1 & 69.93618 & 8.805625 & -1.12423 & 17.49904 \\
\hline P12B7 & 53.76263 & 2.034971 & -1.13119 & 19.9671 \\
\hline P14F7 & 33.10686 & 5.167287 & -1.28679 & 21.95373 \\
\hline P14B 11 & 68.00783 & 7.936257 & -1.29373 & 28.44095 \\
\hline P14A3 & 0.583484 & 15.13281 & -1.37645 & 22.08034 \\
\hline P14A4 & 9.124315 & 35.75554 & -1.41311 & 12.09699 \\
\hline P12E6 & 62.86929 & 18.59965 & -1.56159 & 18.83752 \\
\hline P14H11 & 21.08003 & 26.82509 & -1.62077 & 3.884184 \\
\hline $\mathrm{P} 13 \mathrm{H} 2$ & 29.7638 & 18.18381 & -1.72577 & 21.89342 \\
\hline P14G11 & 11.23583 & 15.66973 & -1.7408 & 4.112302 \\
\hline P12B9 & 54.0754 & 1.867384 & -2.42683 & 18.78046 \\
\hline P13D11 & 22.45504 & 2.907022 & -2.48612 & 28.57463 \\
\hline P12C8 & 55.44219 & 10.50015 & -2.57503 & 15.96577 \\
\hline P13H8 & 75.14213 & 7.815239 & -2.59829 & 21.20335 \\
\hline P14D10 & 28.18139 & 11.26894 & -2.62771 & 16.64255 \\
\hline $\mathrm{P} 12 \mathrm{H} 6$ & 60.53709 & 4.946292 & -2.65674 & 16.26725 \\
\hline
\end{tabular}




\begin{tabular}{|c|c|c|c|c|}
\hline Negative & $-1.6 \mathrm{E}-15$ & $1.04 \mathrm{E}-15$ & -2.74431 & 2.744315 \\
\hline P13F6 & 12.81342 & 38.15991 & -2.82903 & 22.87259 \\
\hline P12F8 & 72.08113 & 6.79015 & -3.06318 & 23.90158 \\
\hline P14A9 & 2.276 & 10.23496 & -3.19931 & 19.05033 \\
\hline P14A6 & 12.33946 & 15.24049 & -3.2443 & 16.26517 \\
\hline P14E10 & 7.978985 & 19.19814 & -3.48289 & 19.87775 \\
\hline P13H1 & 48.59927 & 19.5299 & -3.65957 & 17.3299 \\
\hline P12G9 & 76.25165 & 2.058681 & -3.67557 & 26.36751 \\
\hline P14F1 & 32.11258 & 30.8922 & -4.10634 & 16.84287 \\
\hline P14D9 & 24.72489 & 6.711745 & -4.26301 & 23.80922 \\
\hline P12B12 & 34.76547 & 39.41461 & -4.27627 & 9.297359 \\
\hline P12F7 & 70.16464 & 7.793636 & -4.33108 & 16.42393 \\
\hline P12G2 & 60.79392 & 20.11073 & -4.41381 & 21.2987 \\
\hline P13A9 & 48.55745 & 11.63737 & -5.04958 & 8.261878 \\
\hline P14G12 & 43.73585 & 32.16446 & -5.19446 & 23.06536 \\
\hline P12G8 & 69.28146 & 7.119817 & -5.33807 & 27.94992 \\
\hline P14G9 & 25.99625 & 19.62383 & -5.50316 & 17.5543 \\
\hline P14B6 & 42.80862 & 3.91153 & -5.75594 & 27.53277 \\
\hline P13G7 & 46.48067 & 17.87033 & -5.83064 & 25.21456 \\
\hline P14E3 & 11.85072 & 6.362463 & -5.90241 & 20.92926 \\
\hline P13A10 & 66.15942 & 8.439018 & -6.01168 & 25.72006 \\
\hline P13H3 & 12.10762 & 25.54477 & -6.23194 & 21.08624 \\
\hline P14H7 & -7.08517 & 17.56731 & -6.30445 & 20.40175 \\
\hline P12G4 & 75.53395 & 3.990711 & -6.44333 & 25.79118 \\
\hline P12B2 & 51.80511 & 11.88245 & -6.79739 & 19.93671 \\
\hline P12G3 & 78.85571 & 3.758784 & -6.98983 & 25.07222 \\
\hline P12G6 & 74.16993 & 4.408778 & -7.33904 & 27.2574 \\
\hline P12G10 & 74.40513 & 4.569056 & -7.37694 & 27.74614 \\
\hline P12C9 & 51.26161 & 14.80368 & -7.39038 & 37.42863 \\
\hline P13A11 & 36.57503 & 20.32198 & -7.66617 & 23.99213 \\
\hline P12E4 & 53.62801 & 11.61941 & -7.94973 & 8.836291 \\
\hline P13E4 & 65.3128 & 14.90984 & -8.39176 & 23.83041 \\
\hline P13F11 & 66.52103 & 1.827431 & -8.51001 & 25.53271 \\
\hline P13B12 & 70.02164 & 4.281268 & -8.9044 & 2.921295 \\
\hline $\mathrm{P} 12 \mathrm{H} 3$ & 45.39753 & 39.3663 & -9.0057 & 14.54912 \\
\hline P12H11 & 69.3944 & 9.255719 & -9.23519 & 26.14936 \\
\hline P14D4 & 18.07649 & 29.50043 & -9.58947 & 24.40286 \\
\hline P12G5 & 75.83877 & 3.947214 & -10.0791 & 28.68455 \\
\hline P13A12 & 47.13184 & 0.562767 & -10.547 & 8.076361 \\
\hline P13B8 & 69.55005 & 1.087336 & -10.9057 & 15.64739 \\
\hline P13E1 & 72.67067 & 9.20003 & -11.3864 & 9.612788 \\
\hline P13G12 & 56.09426 & 9.868374 & -11.6851 & 7.481183 \\
\hline P12F11 & 70.29524 & 1.079568 & -12.1406 & 25.01625 \\
\hline P12A4 & 57.60193 & 10.34467 & -12.5505 & 9.768548 \\
\hline P13A3 & 49.4022 & 15.62358 & -13.9029 & 28.20824 \\
\hline P12B3 & 61.98406 & 11.2399 & -14.0016 & 30.86147 \\
\hline
\end{tabular}




\begin{tabular}{lrrrr}
\hline P13A7 & 34.23005 & 14.97206 & -14.9375 & 25.28122 \\
P12A2 & 50.93835 & 8.329339 & -15.6527 & 31.23382 \\
P12F9 & 42.43112 & 15.97481 & -17.0093 & 15.5608 \\
P13A6 & 62.16428 & 4.181207 & -17.1228 & 44.78536 \\
P13A2 & 47.25156 & 10.37642 & -17.8695 & 28.98473 \\
P12E12 & 56.76999 & 7.364205 & -18.9406 & 3.793946 \\
P13A1 & 46.68483 & 2.310021 & -19.1867 & 13.46318 \\
P13F12 & 76.88013 & 0.700357 & -19.2921 & 10.16751 \\
P12E10 & 29.92259 & 37.12848 & -19.8402 & 12.0464 \\
P13D12 & 55.02205 & 12.21098 & -20.6057 & 13.36992 \\
P13C12 & 47.46493 & 29.56519 & -20.8498 & 6.125715 \\
P12H1 & 48.19581 & 9.363045 & -23.1262 & 24.56357 \\
P13H12 & 12.01996 & 17.51483 & -23.9993 & 23.24625 \\
P13G2 & 16.64483 & 19.67116 & -26.3061 & 32.82964 \\
P12F12 & 44.35305 & 12.37506 & -28.4174 & 16.9287 \\
P12F1 & 47.2474 & 11.47322 & -29.6369 & 14.3363 \\
P12A7 & 44.48769 & 14.79392 & -31.7808 & 6.799194 \\
P12G1 & 69.45505 & 3.245193 & -31.8402 & 7.978208 \\
P12C12 & 62.69667 & 16.04693 & -31.9423 & 11.03574 \\
P13D4 & 61.82762 & 12.19567 & -33.2304 & 36.61669 \\
P12D1 & 48.25377 & 20.44374 & -33.4047 & 12.65876 \\
P12C1 & 65.50274 & 7.063881 & -37.7655 & 16.60946 \\
P12A12 & 38.54574 & 8.3305 & -39.7585 & 8.580804 \\
P12H12 & 12.05098 & 24.78073 & -45.1262 & 40.06738 \\
P12D12 & 51.24517 & 6.066775 & -49.5603 & 27.39543 \\
P13G1 & 38.28933 & 19.71935 & -51.7756 & 22.4385 \\
P12A1 & 23.71434 & 11.1765 & -54.5519 & 54.92307 \\
P13C1 & 28.31176 & 33.08247 & -57.5063 & 58.08748 \\
\hline & & & & \\
& & & & \\
\hline
\end{tabular}




\section{VUW MNP library screening raw data}

\section{Table 7.2 Raw data of VUW MNP library screen vs. M. smegmatis}

Top hits highlighted in yellow, positive control in red and negative control in green. Plates RAK18_52A/B, 53A/B and 53C/D were screened against $M$. smegmatis as stated earlier RAK18_52A/B was not screened in duplicate therefore no standard deviation (std dev) value are listed for extracts from this plate. Std dev is used instead of standard error as standard error values is applicable for triplicate data.

RAK18_52 A/B represented as RAK1852 followed by the respective well number.

RAK18_53A/B represented as RAK18A followed by the respective well number.

RAK18_53C/D represented as RAK18C followed by the respective well number.

\begin{tabular}{lrlrl}
\hline Well number & \multicolumn{1}{l}{$\begin{array}{l}\text { GFP } \\
\text { average }\end{array}$} & $\begin{array}{l}\text { GPF } \\
\text { std dev }\end{array}$ & $\begin{array}{l}\text { Resazurin } \\
\text { average }\end{array}$ & $\begin{array}{l}\text { Resazurin } \\
\text { std dev }\end{array}$ \\
\hline RAK1852D5 & 82.51134 & & 86.33764 & \\
RAK1852D7 & 82.29852 & & 84.95481 & \\
RAK1852G6 & 81.28371 & & 84.82935 & \\
RAK1852H7 & 81.93423 & & 84.08974 & \\
RAK1852B5 & 82.48722 & & 83.98016 & \\
RAK1852G2 & 83.40519 & & 83.86051 & \\
RAK1852D8 & 82.61491 & & 83.76624 & \\
RAK18CH8 & 88.25275 & 2.492621 & 80.87763 & 0.734711 \\
RAK18AD7 & 87.31818 & 0.149184 & 78.43139 & 1.641461 \\
Positive & 86.92569 & 2.794045 & 78.12862 & 5.72208 \\
RAK18AA11 & 85.72099 & 0.54014 & 74.28695 & 0.237072 \\
RAK18AE6 & 78.32324 & 8.843107 & 52.21352 & 27.78748 \\
RAK1852G12 & 78.8809 & & 41.2392 & \\
RAK18CG8 & 62.80922 & 24.57021 & 39.83496 & 45.13486 \\
RAK1852G1 & 62.75679 & & 31.20559 & \\
RAK18CC10 & 60.35443 & 13.82721 & 26.96934 & 34.5404 \\
RAK1852G9 & 43.6266 & & 22.82428 & \\
RAK18CF10 & 54.17289 & 13.87771 & 21.88638 & 7.693616 \\
RAK1852G7 & 31.59895 & & 21.81058 & \\
RAK18CD7 & 49.98409 & 15.10035 & 21.58811 & 1.318869 \\
RAK1852G3 & 44.64637 & & 21.58543 & \\
RAK1852E9 & 37.43063 & & 21.04569 & \\
RAK1852C9 & 61.86191 & & 20.79466 & \\
RAK1852H12 & 56.28451 & & 20.73401 & \\
RAK18CF7 & 39.27054 & 16.71114 & 19.8724 & 7.734189 \\
\hline
\end{tabular}




\begin{tabular}{|c|c|c|c|c|}
\hline $\mathrm{RAF}$ & 43.65879 & 12.61971 & 18.84471 & 7.782954 \\
\hline RAK1852F8 & 41.61507 & & & \\
\hline AK18CC4 & .4603 & 6.25646 & 0972 & 0.086892 \\
\hline RAK1852G8 & 47.00231 & & & \\
\hline RAK18CE4 & 47.10365 & 16.12104 & 17.40278 & 4.279494 \\
\hline D6 & 234 & 15.32 & & 9.32 \\
\hline RAK18CD11 & 39.32563 & 8.246603 & & 4.730476 \\
\hline & 45.7 & 18.35168 & & 10.01583 \\
\hline RAK1852G11 & 43.14526 & & & \\
\hline & 25.9 & 39.92709 & & 971 \\
\hline & 34.6 & 6.208976 & & \\
\hline & 28.8 & 23. & & 278 \\
\hline & 61.4 & 6.629442 & & \\
\hline RAK & 84 & 5.974945 & & 3.8 \\
\hline & 30.2 & & & \\
\hline $2 \mathrm{C} 7$ & 41.6 & & & \\
\hline & & 29. & & 259 \\
\hline E11 & 48.4 & 2.06 & & 086 \\
\hline & & 9.86 & & \\
\hline & & & & \\
\hline & 3 & 18.3 & & 791 \\
\hline & & & & \\
\hline RA & 67 & 9.555635 & & 2.334622 \\
\hline $\mathrm{F} 3$ & 18.9 & & & \\
\hline $\mathrm{J} 3$ & 19 & 14. & & 189 \\
\hline & & 19.6 & & 898 \\
\hline D9 & -0.42405 & & & \\
\hline $\mathrm{F} 2$ & -2.5 & & & \\
\hline & & & & \\
\hline & 4 & 8.91691 & & $8.5^{\prime}$ \\
\hline RA & 43.6 & & & \\
\hline & 39.0 & 97 & & 23 \\
\hline $\mathrm{RA}$ & 3 & 10.2 & 31 & 592 \\
\hline & & 4.672 & & \\
\hline & 3 & 21.4 & & 981 \\
\hline & & 29.82615 & & \\
\hline & 37.9 & 17.9 & & 292 \\
\hline & & 14.30857 & & 5.333487 \\
\hline $2 \mathrm{H} 2$ & 26.7 & & & \\
\hline RAK & & & & \\
\hline & 29.96592 & 0.952737 & & 3.123463 \\
\hline RA & 33.1 & 19.5 & 01 & 0.764271 \\
\hline & 48.80099 & 9.638707 & 12.03279 & 8.855017 \\
\hline RAK1852H5 & 34.93849 & & & \\
\hline & & & & \\
\hline RAK18CB4 & 37.25737 & 12.81713 & 11.82043 & 2.358022 \\
\hline
\end{tabular}




\begin{tabular}{|c|c|c|c|c|}
\hline RAK1852A9 & 13.5793 & & 11.48219 & \\
\hline RAK18CE5 & 40.57995 & 16.65375 & 11.30892 & 01 \\
\hline RAK18CC1 & 54.5389 & 6.189958 & & 2.06 \\
\hline RAK18CB9 & 24.35392 & 5.51662 & 11.14215 & \\
\hline RAK18CE2 & 35.62878 & 3.671658 & 11.06653 & 5.279631 \\
\hline RAK1852C4 & 44.99575 & & $10.8^{\circ}$ & \\
\hline RAK18CB8 & 42.4589 & 12.07647 & 10.72786 & 0.043497 \\
\hline RAK1852C8 & 25.35723 & & 10.70004 & \\
\hline RAK18CG2 & 23.10204 & 24.0 & 54 & 2.620404 \\
\hline 8AD11 & 6.681053 & 31.8454 & 997 & 10.6675 \\
\hline RAK1852G4 & 43.38859 & & 28 & \\
\hline RAK18AD8 & 42.50065 & 1.77 & 33 & 189 \\
\hline RAK18CE7 & 44.62251 & 22.5 & & \\
\hline RAK18AF7 & 48.19767 & 16.4 & 69 & 9.6 \\
\hline 18CF11 & 11 & 3.66 & & \\
\hline RAK18CC11 & 23.73454 & 13.2 & 65 & $1.3^{\circ}$ \\
\hline & 39.47 & 20.2 & & \\
\hline RAK1852H3 & 35.3471 & & 177 & \\
\hline B10 & 3503 & & & \\
\hline G10 & & & & \\
\hline RAI & 7.20761 & 11. & & 824 \\
\hline H10 & & & & \\
\hline RAK1 & 81 & 34. & 36 & 083 \\
\hline & 91 & 30. & 43 & 343 \\
\hline $2 \mathrm{~B} 7$ & 54.1 & & & \\
\hline $2 \mathrm{~A} 10$ & 28.7 & & & \\
\hline RAK18AH4 & 26.33 & 19.60195 & & 14.93681 \\
\hline $\mathrm{H} 2$ & & 2.192368 & 24 & 6.578632 \\
\hline & & & & \\
\hline $\mathrm{RA}$ & 93 & 12. & & 748 \\
\hline RA & & & & 876 \\
\hline & & 12.9 & & 8098 \\
\hline RA & 23 & 0.789772 & 25 & 2.268652 \\
\hline RAK1852G5 & 34.29895 & & & \\
\hline E7 & 51.6 & & 03 & \\
\hline & 46.76334 & 24.68317 & & 4.128095 \\
\hline $2 \mathrm{H} 11$ & 23.93061 & & 226 & \\
\hline RAK18CA3 & 29.31859 & 13.31 & & \\
\hline & 16.4 & & 16 & 107 \\
\hline & & & & 8.886059 \\
\hline & 37.28179 & 14.72419 & 7.222739 & 1.578026 \\
\hline RAK & 31.27687 & 5.289814 & 7.057244 & 3.935705 \\
\hline $2 \mathrm{E} 4$ & 37.31997 & & & \\
\hline RAK18CG4 & 34.74121 & 15.3 & 6.984139 & 1.92 \\
\hline & 21.73172 & 11.09809 & 6.981507 & 10.75625 \\
\hline RAK1852A3 & 23.35777 & & 6.957131 & \\
\hline
\end{tabular}




\begin{tabular}{|c|c|c|c|c|}
\hline RAK18CH5 & 33.66798 & 14.86288 & 6.942604 & 6.816235 \\
\hline RAK18CA5 & 4.44111 & 15.19794 & 589 & 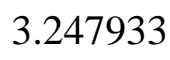 \\
\hline & 219 & 15 & & 0.070078 \\
\hline RAK18AF3 & 22.40652 & 7.444122 & & 13.23768 \\
\hline RAK1852H4 & 43.34248 & & 6.668901 & \\
\hline RAK18AG5 & 43.12163 & 4.664573 & 6.612338 & 6.895605 \\
\hline RAK18CA1 & 60.67739 & 5.464107 & 6.417738 & 11.39781 \\
\hline RAK18AH6 & 24.6 & 15.1173 & 117 & 6.603738 \\
\hline RAK18CA4 & 27.5695 & 22.09493 & 6.350291 & 6.073261 \\
\hline & 45.46937 & 0.591618 & & 10.67808 \\
\hline RAK18CE9 & 14.61035 & 40.4074 & 6.2 & 1.603988 \\
\hline 1 & 42 & 7.43 & & 7392 \\
\hline $\mathrm{C} 12$ & 53. & 1.71 & & 2.523299 \\
\hline RAK18. & 33.9 & & 378 & \\
\hline RAK18AG12 & & 51 & & 379 \\
\hline RAK18 & 35.50482 & 472 & 387 & 11.27223 \\
\hline RAK1852F10 & 9.737147 & & & \\
\hline RAK1 & 75 & 206 & 018 & 328 \\
\hline RAK18 & & & & \\
\hline RAK18 & 36.5 & & 5.7 & \\
\hline B9 & & & & \\
\hline & & & & \\
\hline 11 & & 08 & & 874 \\
\hline & & & & \\
\hline RAK1 & 32.5208 & 16.52901 & 5.528628 & 3.723113 \\
\hline $\mathrm{F} 11$ & 30.86223 & & 91 & \\
\hline RAK18 & 1.22 & 45. & 21 & 3.660894 \\
\hline RAK18 & 51 & 38 & 92 & 427 \\
\hline & & 23. & & 2.879564 \\
\hline RA & 02 & 1.0 & 08 & 13.0501 \\
\hline & & & & \\
\hline & & & & 285 \\
\hline & & & & 323 \\
\hline & & 10.5 & & 2.841401 \\
\hline RAK18AD9 & 1.142218 & 7.697591 & 4.7 & 1.883591 \\
\hline RAK1852D10 & 33.72469 & & 4.608182 & \\
\hline RAK18 & 30.5 & 4.00 & 599 & 7103 \\
\hline & 40.74942 & 17.08258 & 4.3 & 0.981454 \\
\hline $2 \mathrm{~A} 12$ & 36.23474 & & 4.3 & \\
\hline CG12 & 48.34821 & 1.77 & 4.1 & 2.055925 \\
\hline RAK18CA12 & 45.6 & 3.622087 & 4.1 & 1.060737 \\
\hline & & & & 6.954887 \\
\hline & 26.46577 & 10.15487 & 3.902579 & 1.996798 \\
\hline RAK1852F6 & 31.71352 & & 3.816351 & \\
\hline & 42.90091 & 12.06179 & 3.642459 & 8.126461 \\
\hline RAK1852B10 & 32.85283 & & 3.559221 & \\
\hline
\end{tabular}




\begin{tabular}{|c|c|c|c|c|}
\hline RAK18AE4 & 36.00865 & 13.30036 & 3.5313 & 3.035798 \\
\hline RAK18CA11 & 35.08207 & 0.156968 & 11606 & 009 \\
\hline RAK18CC3 & 9.339796 & 21.06663 & 06 & \\
\hline RAK18CE12 & 56.49319 & 9.311254 & 3.257728 & \\
\hline RAK1852D11 & 28.21366 & & 3.247062 & \\
\hline & 43 & 10.56111 & 01 & 558 \\
\hline RAK1852A11 & 13.27461 & & 3.212668 & \\
\hline & 20.5 & 3.10 & 3.18032 & 504 \\
\hline RAK18CH6 & 2.026747 & 2.65 & 2.974 & 542 \\
\hline & & 13. & 2.922 & 708 \\
\hline & 33.1 & 11.3 & 2.83 & 677 \\
\hline & 22. & 4.2 & 2.7 & 5249 \\
\hline & & 0.586597 & & 5.538246 \\
\hline RAK1852H10 & 32.0 & & 2.6427 & \\
\hline & 26.0 & 15. & & 434 \\
\hline RA & & 17.59309 & & 224 \\
\hline & & & & \\
\hline $\mathrm{RAl}$ & 36.5 & 5.75 & 2.4 & 247 \\
\hline & & & & 242 \\
\hline & & & & \\
\hline & 8 & & & 686 \\
\hline & & & & \\
\hline 10 & 69 & & & 488 \\
\hline & & & & 163 \\
\hline & 27 & & & 987 \\
\hline & & & & 169 \\
\hline & & & & 813 \\
\hline & & & & 827 \\
\hline & & & & 534 \\
\hline & & & & \\
\hline & & & & \\
\hline RA & & 23.87637 & 0.65 & 0.39497 \\
\hline $\mathrm{F} 4$ & 20.2 & & & \\
\hline & & 0.59591 & 0.53 & $0.1^{\prime}$ \\
\hline & 19.1 & & 0.46 & \\
\hline & & & & \\
\hline RA & 31.7 & & 0.2 & \\
\hline & & & & \\
\hline RA & 37.37279 & & 0.056468 & 0.418971 \\
\hline & & & $2.84 \mathrm{E}-15$ & $3.89 \mathrm{E}-15$ \\
\hline & & & & 7.400697 \\
\hline RA & 24.00114 & 6.522 & -0.18 & 4.742422 \\
\hline & & & & 762 \\
\hline RAK18AE1 & 27.51207 & & -0.28578 & 4.21642 \\
\hline & & 0.85 & & \\
\hline RAK18AF4 & 27.20402 & 14.37533 & -0.5966 & 20.17942 \\
\hline
\end{tabular}




\begin{tabular}{|c|c|c|c|c|}
\hline RAK18AF9 & 18.09764 & 24.6521 & -0.67438 & 11.77507 \\
\hline RAK18AB6 & 46.21676 & 0.334301 & -0.75874 & \\
\hline RAK18AD1 & 29.46976 & 18.97909 & -0.86904 & 1.072651 \\
\hline RAK18AA6 & 9.8147 & 0.584787 & & 1276 \\
\hline RAK18AG4 & 37.55022 & 0.01991 & -1.01646 & 3.350942 \\
\hline RAK18AB 10 & 38 & 11.7 & 203 & 10.6011 \\
\hline RAK18AD12 & 21.25354 & 7.169368 & -1.2628 & 1.676767 \\
\hline RAK18AA7 & 39.52037 & 13.7623 & & 0.724543 \\
\hline RAK1852E1 & 32.45254 & & -1.50761 & \\
\hline RAK18 & 35.7 & 21.90377 & & 5.884542 \\
\hline RAK18AE8 & 41.7 & 9.733895 & & 2196 \\
\hline RAK1 & 31 & 54.80276 & & 7051 \\
\hline RAK1852A6 & 24.64428 & & & \\
\hline RAK18AH9 & 18.17687 & 43.7 & -1.5 & 514 \\
\hline & & 13.0 & & 503 \\
\hline RAK1852B6 & 35.4 & & -2.0 & \\
\hline & & & & \\
\hline RAK1 & 14.0 & & -2 & \\
\hline $\mathrm{RA}$ & 38.8 & & & \\
\hline & & 37.56784 & & 0564 \\
\hline RAK1852H1 & 20.79624 & & -2.5 & \\
\hline & & & & .700842 \\
\hline RAK 1 & & 19.9 & & 1937 \\
\hline H10 & 57 & 0.79 & & 2771 \\
\hline $\mathrm{C} 10$ & 2 & 2.99 & & 402 \\
\hline & 29 & 8.82 & & 111 \\
\hline RA & 32.9 & 5.67 & & 11.01093 \\
\hline & & 13. & & 073 \\
\hline & & & & \\
\hline RA & 6 & 16.5 & & 713 \\
\hline RAK18 & 8 & & 41 & 5065 \\
\hline $\mathrm{H} 12$ & 13 & 9.54 & -4 & 9.542489 \\
\hline RAK & 22 & 14.7 & 28 & 383 \\
\hline & 34.45813 & 5.627901 & -4.2 & 16.53235 \\
\hline RA & 2.67 & & -4 & \\
\hline & 32.71475 & 10. & & \\
\hline RAK18AC11 & 27.25304 & 0.372096 & -4.8 & 0.409552 \\
\hline RAK18CB1 & 17.40274 & 16.69361 & 56 & 13.15581 \\
\hline G10 & & 16.19178 & 62 & 0.9 \\
\hline RAK18AC1 & 25.46507 & 8.67867 & -5.29521 & 7.674458 \\
\hline RAK1852D12 & 19.96291 & & & \\
\hline RAK1852C1 & 46.85089 & & -5.6 & \\
\hline RAK18AC5 & 28.45948 & 8.570964 & -6.33864 & 19.13271 \\
\hline RAK1852A4 & 9.629672 & & -6.43095 & \\
\hline & 18.58409 & 1.197894 & -6.63394 & 2.948968 \\
\hline RAK1852C2 & 39.57375 & & -6.73817 & \\
\hline
\end{tabular}




\begin{tabular}{lrrrr}
\hline RAK18AB1 & 24.96809 & 10.69279 & -6.89769 & 0.527962 \\
RAK18AC2 & 24.18161 & 8.432652 & -7.29601 & 6.497312 \\
RAK18AB7 & 33.56221 & 29.0952 & -7.36759 & 20.2746 \\
RAK18AH11 & -6.39996 & 24.00148 & -7.50226 & 14.69246 \\
RAK18AB2 & 33.68643 & 9.540991 & -7.86444 & 9.194541 \\
RAK18CH1 & 22.20906 & 11.19038 & -8.68446 & 1.955926 \\
RAK18AA2 & 7.372359 & 9.452771 & -9.21463 & 2.771314 \\
RAK18AC7 & 15.8392 & 39.01105 & -9.67571 & 20.9994 \\
RAK1852F12 & 26.92394 & & -10.3629 & \\
RAK18AH8 & 27.36875 & 10.97076 & -10.8755 & 17.81443 \\
RAK18CF12 & 23.06386 & 14.98629 & -11.641 & 0.434402 \\
RAK1852E2 & 59.21263 & & -20.1123 & \\
RAK1852E10 & 32.41725 & & -24.7939 & \\
RAK1852D1 & 66.17376 & & -25.3368 & \\
RAK1852F5 & 46.0063 & & -25.8884 & \\
RAK1852B11 & 33.04011 & & -28.8941 & \\
RAK18AG8 & 45.88341 & 15.66059 & -29.7372 & 16.09452 \\
RAK1852B8 & 53.89066 & & -29.9097 & \\
RAK1852D6 & 62.61566 & & -30.8714 & \\
RAK1852A8 & 42.47381 & & -32.3404 & \\
RAK1852D2 & 64.59845 & & -33.3008 & \\
RAK1852B2 & 54.27409 & & -33.5476 & \\
RAK1852C5 & 59.30343 & & -33.5801 & \\
RAK1852D3 & 64.28844 & & -33.8877 & \\
RAK1852B3 & 40.79783 & & -34.6267 & \\
RAK1852F7 & 62.8487 & & -35.4226 & \\
RAK1852C3 & 62.76392 & & -35.9771 & \\
RAK1852E5 & 62.48796 & & -37.2181 & \\
RAK1852B4 & 62.5174 & & -38.3427 & \\
RAK1852C10 & 58.65113 & & -38.7962 & \\
RAK1852E11 & 58.51209 & & -39.3114 & \\
RAK1852E12 & 31.53805 & & -41.141 & \\
RAK1852C11 & 39.84829 & & -41.1927 & \\
RAK1852B12 & 59.0764 & & -52.5119 & \\
RAK1852C12 & 57.55604 & & -53.5649 & \\
\hline & & & & \\
& & & \\
\end{tabular}




\title{
Increased resistance of stationary phase mycobacteria
}

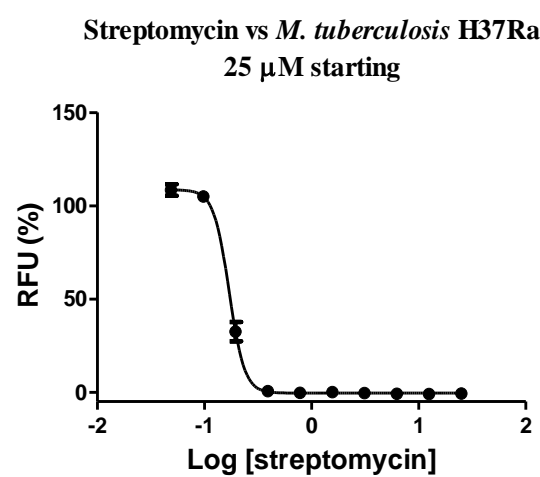

A

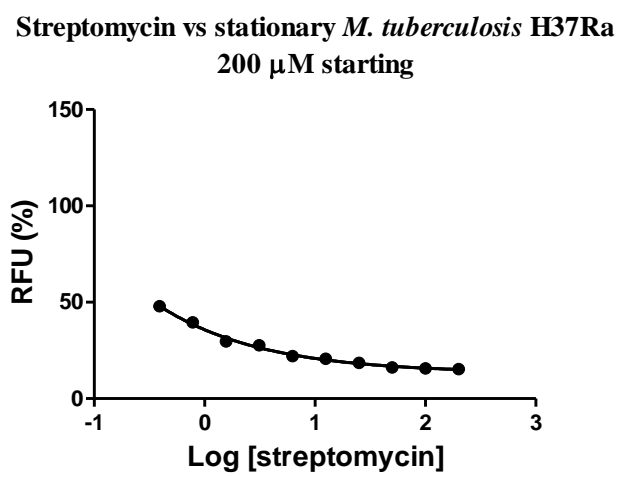

B

\begin{abstract}
A) dose response curve of streptomycin in a growth inhibition assay. B) dose response curve of streptomycin against stationary phase H37Ra. 99\% inhibition is observed at $0.4 \mu \mathrm{M}$ of streptomycin in $\log$ phase $\mathrm{H} 37 \mathrm{Ra}(\mathbf{A})$, however at this concentration there is still $50 \%$ growth observed in stationary phase bacteria $(\mathbf{B})$. Graph generated using GraphPad Prism v.5.0. It is noted that the graph shape for $\mathbf{B}$ is not sigmoidal, however it is still evident that compared to log phase bacteria, stationary phase bacteria are more resistant to inhibiton. This is made evident when observing the fact the even at the starting concentration of $200 \mu \mathrm{M}$ approximately 10 times more than that used for log phase cells, there is still $15 \%$ residual metabolism measure.
\end{abstract}




\section{G2-M block assay}

\begin{tabular}{lrrrrrrrrr}
\hline & Ungated & apoptotic & G0 & G2-M & other & S & total & $\begin{array}{c}\text { G2-M } \\
\text { \% } \\
\text { Sample }\end{array}$ \\
& Count & Count & Count & Count & Count & Count & Count & $\begin{array}{c}\text { compared } \\
\text { to total }\end{array}$ \\
& 10341 & 2038 & 4551 & 1999 & 8303 & 1616 & 8139 & 24.6 \\
\hline Specimen_001_Tube_001.fcs & & & & & & & & \\
& 10414 & 2473 & 5176 & 1455 & 7941 & 1168 & 7805 & 18.6 \\
Specimen_001_Tube_002.fcs & & & & & & & & \\
Specimen_001_Tube_003.fcs & 10428 & 2411 & 4613 & 1705 & 8017 & 1562 & 7875 & 21.7 \\
Mean & $\mathbf{1 0 3 9 4}$ & $\mathbf{2 3 0 7}$ & $\mathbf{4 7 8 0}$ & $\mathbf{1 7 2 0}$ & $\mathbf{8 0 8 7}$ & $\mathbf{1 4 4 9}$ & $\mathbf{7 9 4 0}$ & \\
SD & $\mathbf{4 6 . 7}$ & $\mathbf{2 3 5}$ & $\mathbf{3 4 4}$ & $\mathbf{2 7 2}$ & $\mathbf{1 9 1}$ & $\mathbf{2 4 5}$ & $\mathbf{1 7 6}$ & \\
\hline
\end{tabular}

The samples show no significant difference at any concentration of $\mathbf{1 5 6}$ 


\section{Appendix B: Fatty acid mixture from Psychrolutes marcidus}

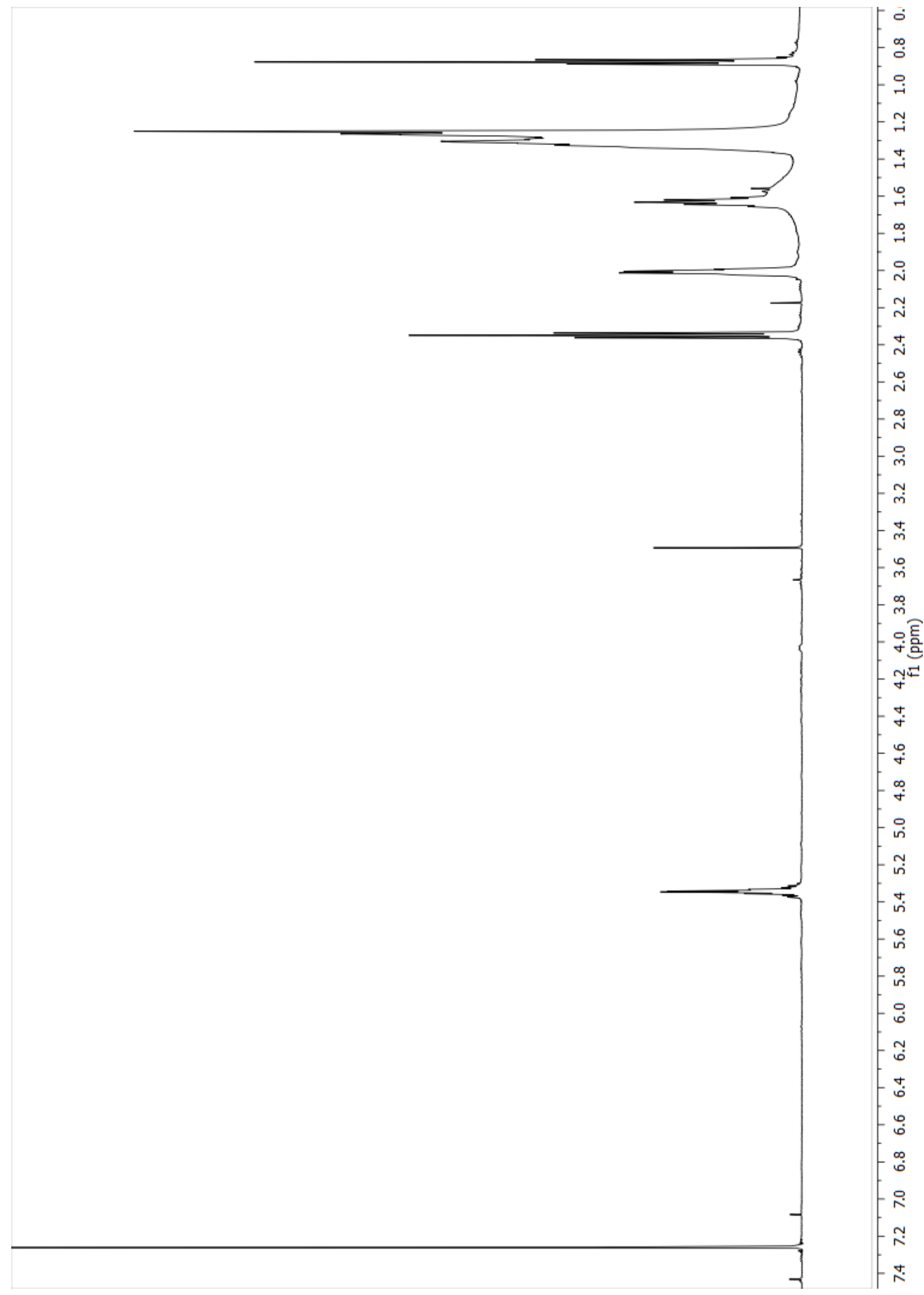

${ }^{1} \mathrm{H} \mathrm{NMR}\left(\mathrm{CDCl}_{3}, 600 \mathrm{MHz}\right)$ spectrum of fatty acid mixture from the $\mathrm{MeOH}$ liver extract of Psychrolutes marcidus 


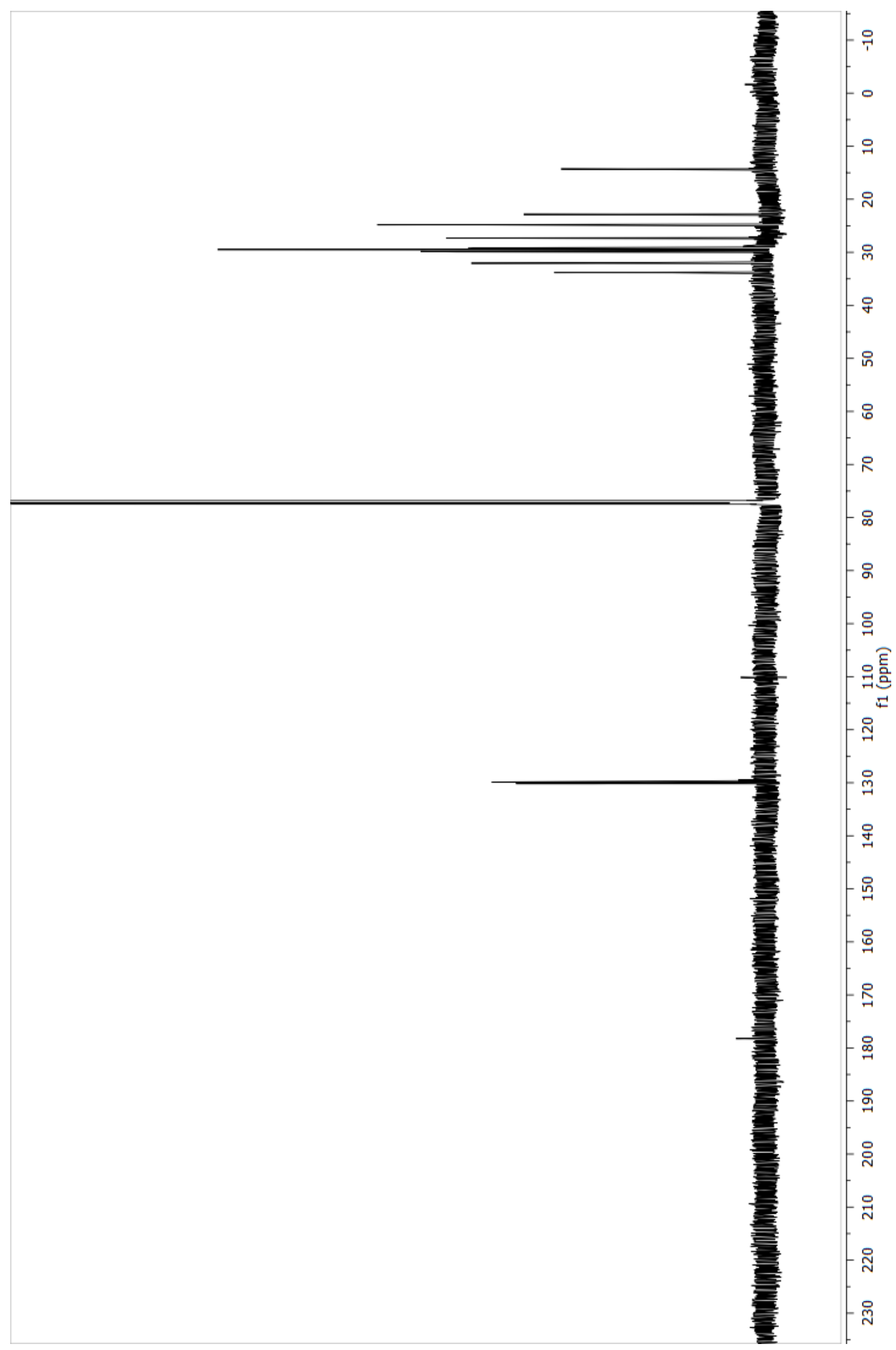

${ }^{13} \mathrm{C} \mathrm{NMR}\left(\mathrm{CDCl}_{3}, 150 \mathrm{MHz}\right)$ spectrum of fatty acid mixture from the $\mathrm{MeOH}$ liver extract of Psychrolutes marcidus 


\section{GC/MS Chromatograms of ND5_96L FAME analysis}

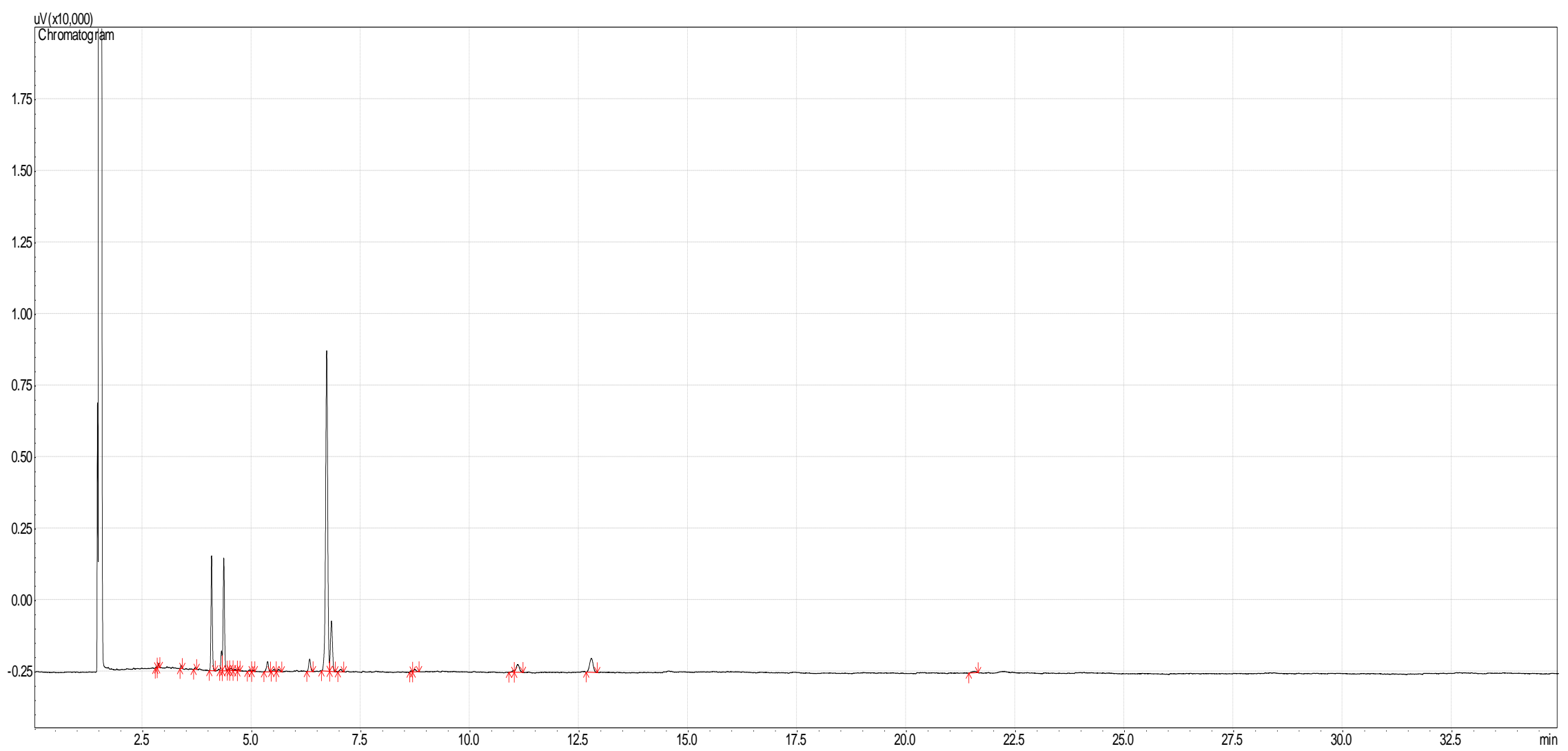

Full GC trace of ND5_96L FAME (Integrated Bioactive Technologies group, Callaghan Innovation). 


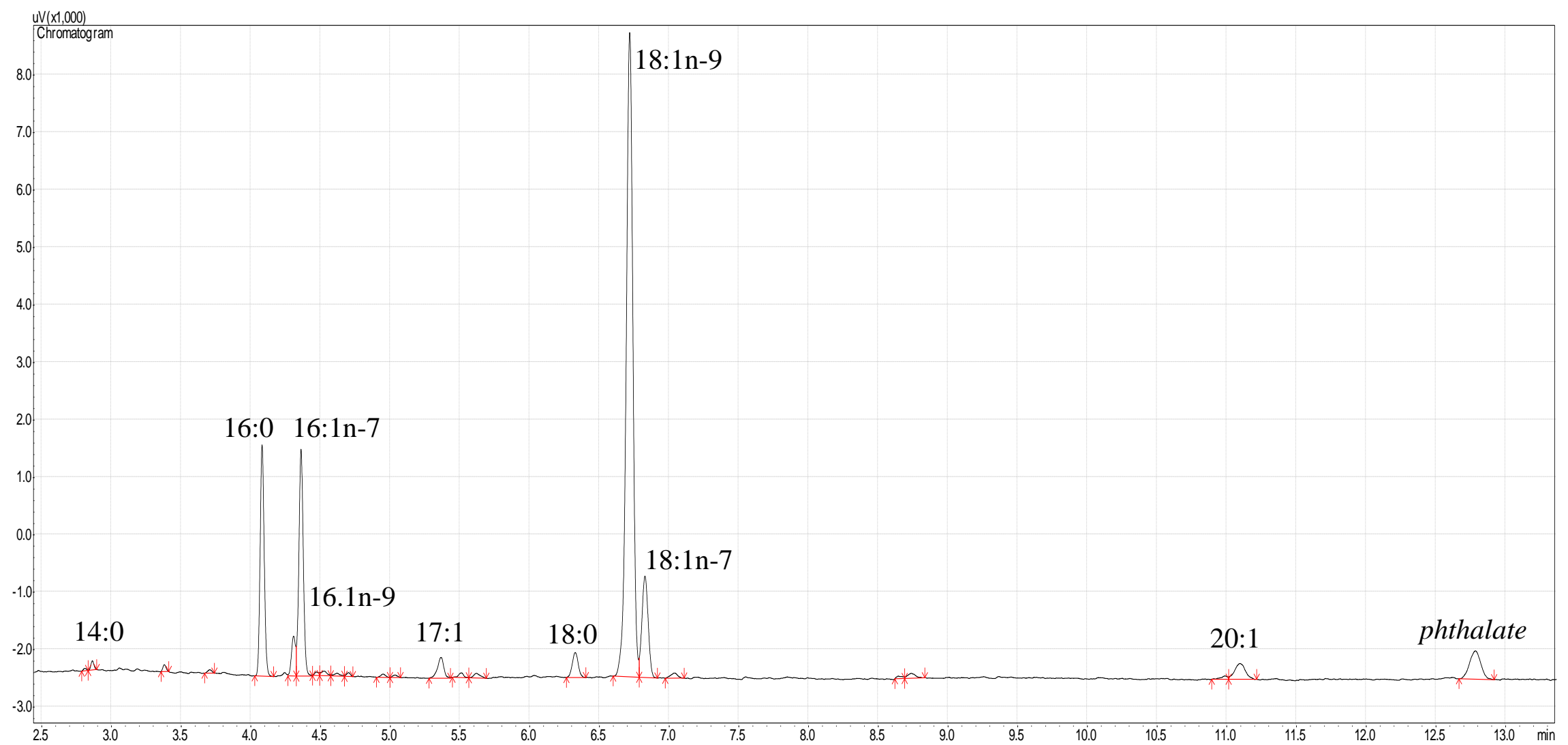

Zoom in of relevant section of GC trace of ND5_96L FAME, with relevant labels for peaks of interest (Integrated Bioactive Technologies group, Callaghan Innovation). 
Appendix C: NMR spectra of rimarikiamide A (98)

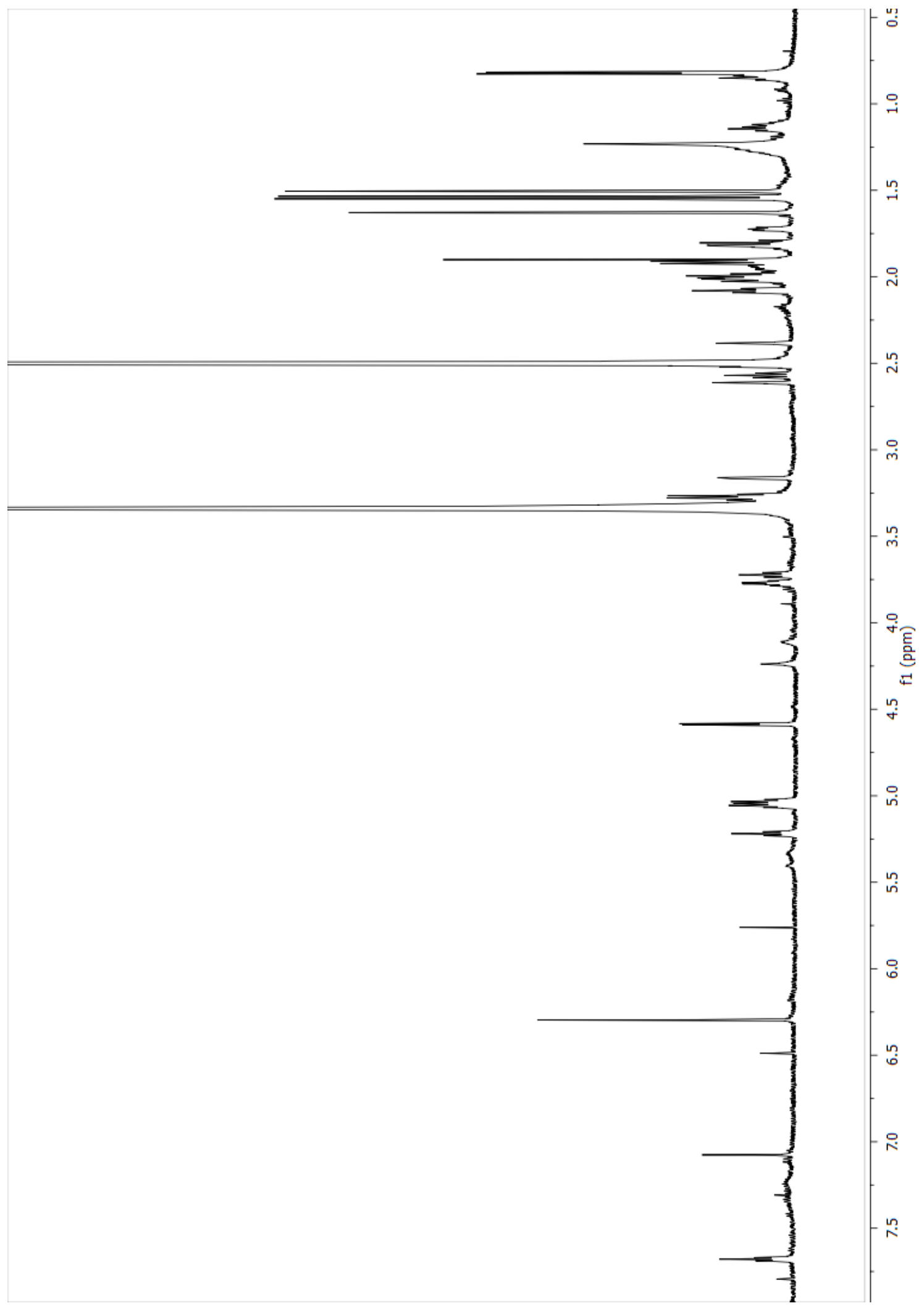

${ }^{1} \mathrm{H}$ NMR (DMSO-d, $600 \mathrm{MHz}$ ) spectrum of rimarikiamide A (98) 


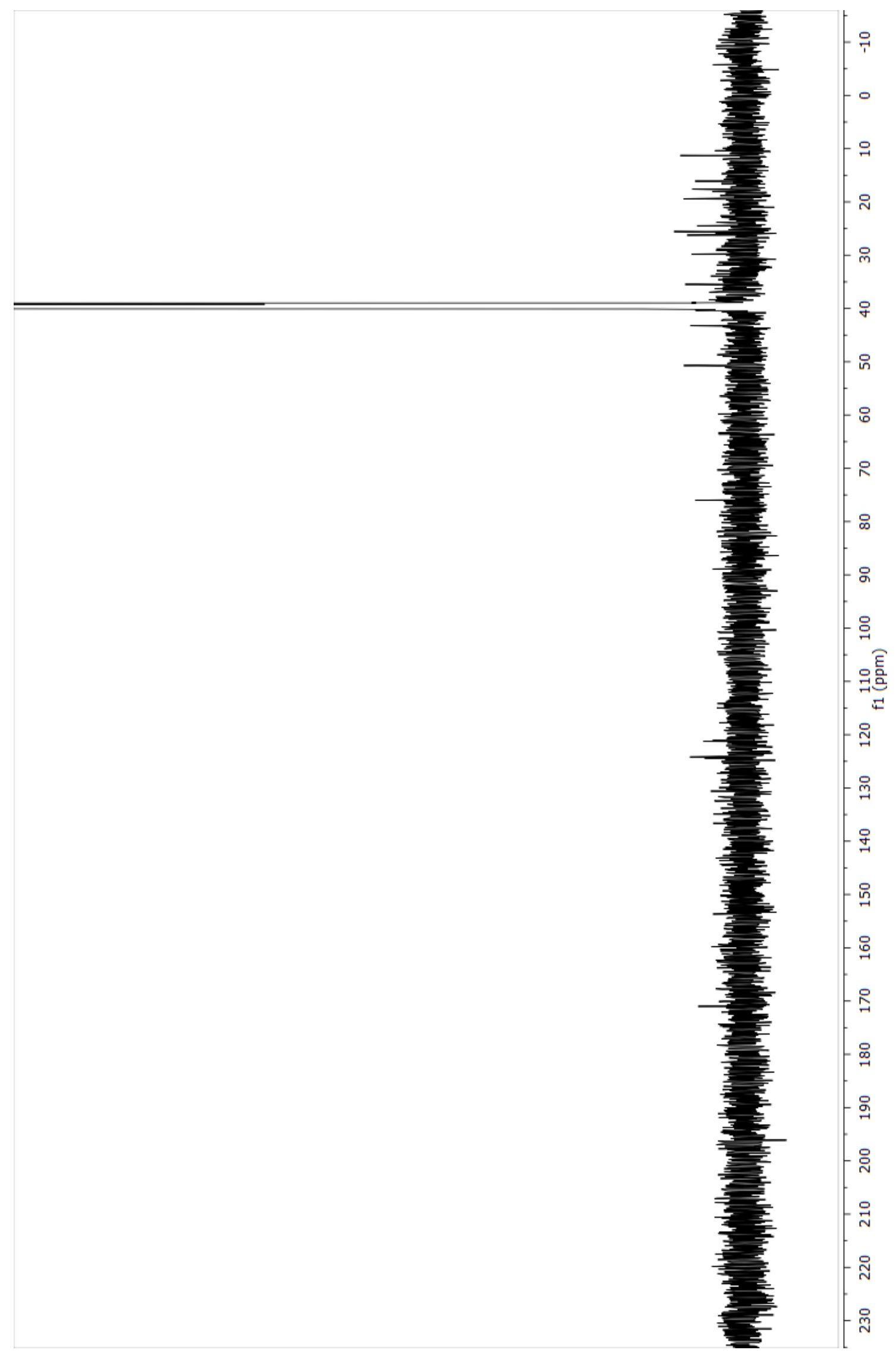

${ }^{13} \mathrm{C}$ NMR $\left(\mathrm{d}_{6} \mathrm{DMSO}, 150 \mathrm{MHz}\right)$ spectrum of rimarikiamide A (98) 
(udd) it

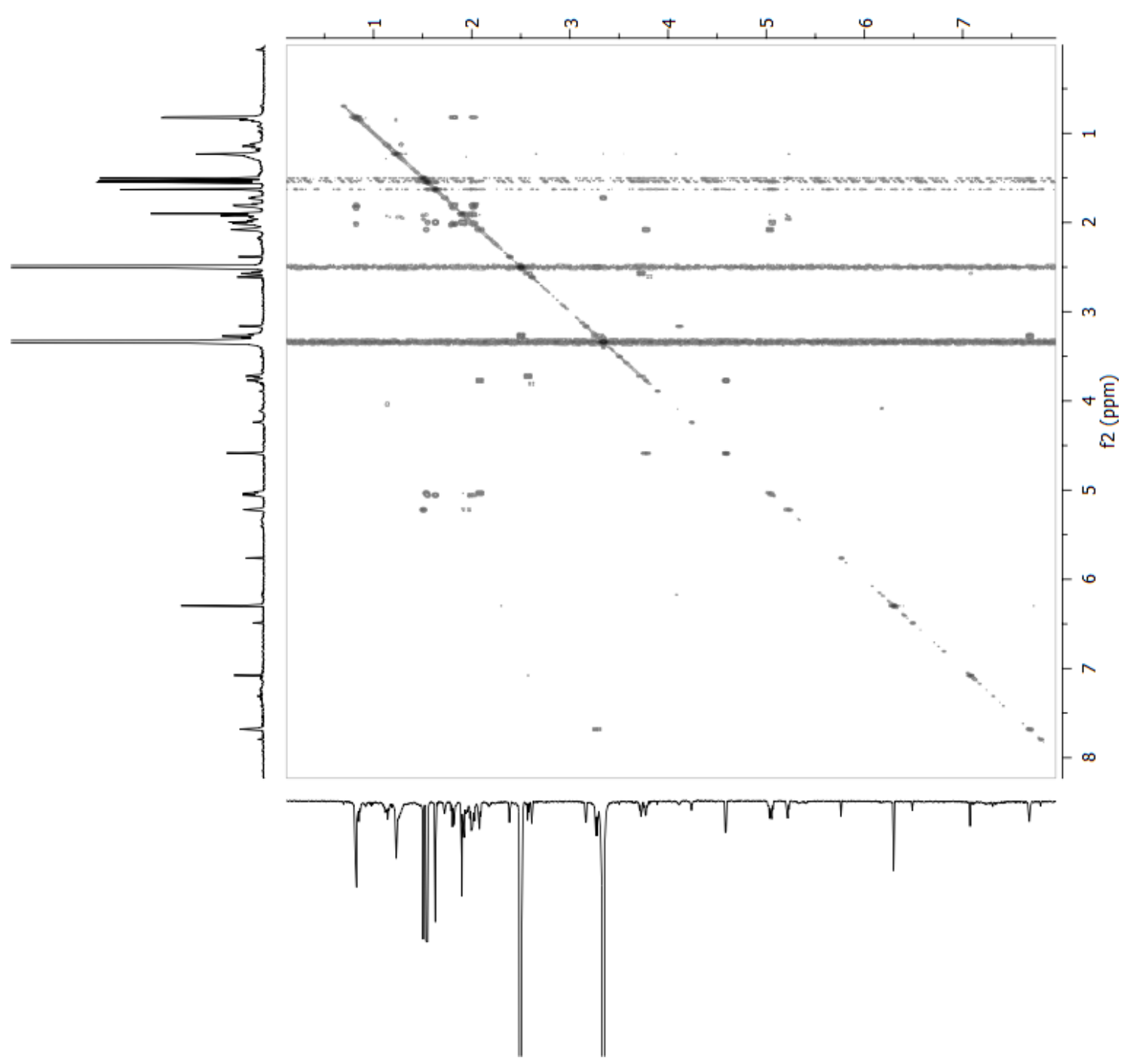

gCOSY NMR (DMSO-d $6,600 \mathrm{MHz}$,) spectrum of rimarikiamide A (98) 
(udd) if

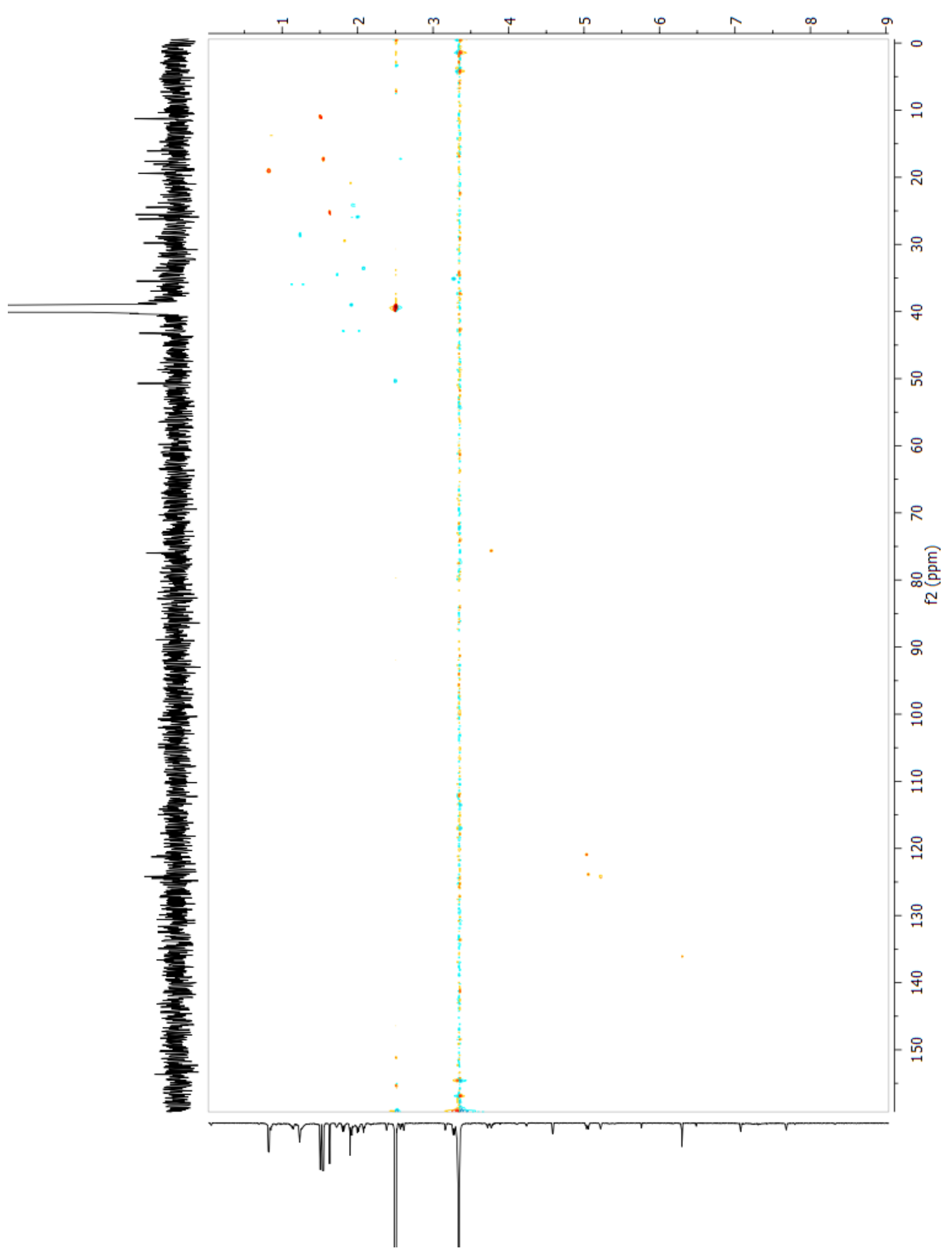

Multiplicity-edited HSQC NMR (DMSO- $\mathrm{d}_{6}, 600 \mathrm{MHz}$,) of rimarikiamide A (98) showing methylenes in blue and methyls and methines in red. 
(udd) is

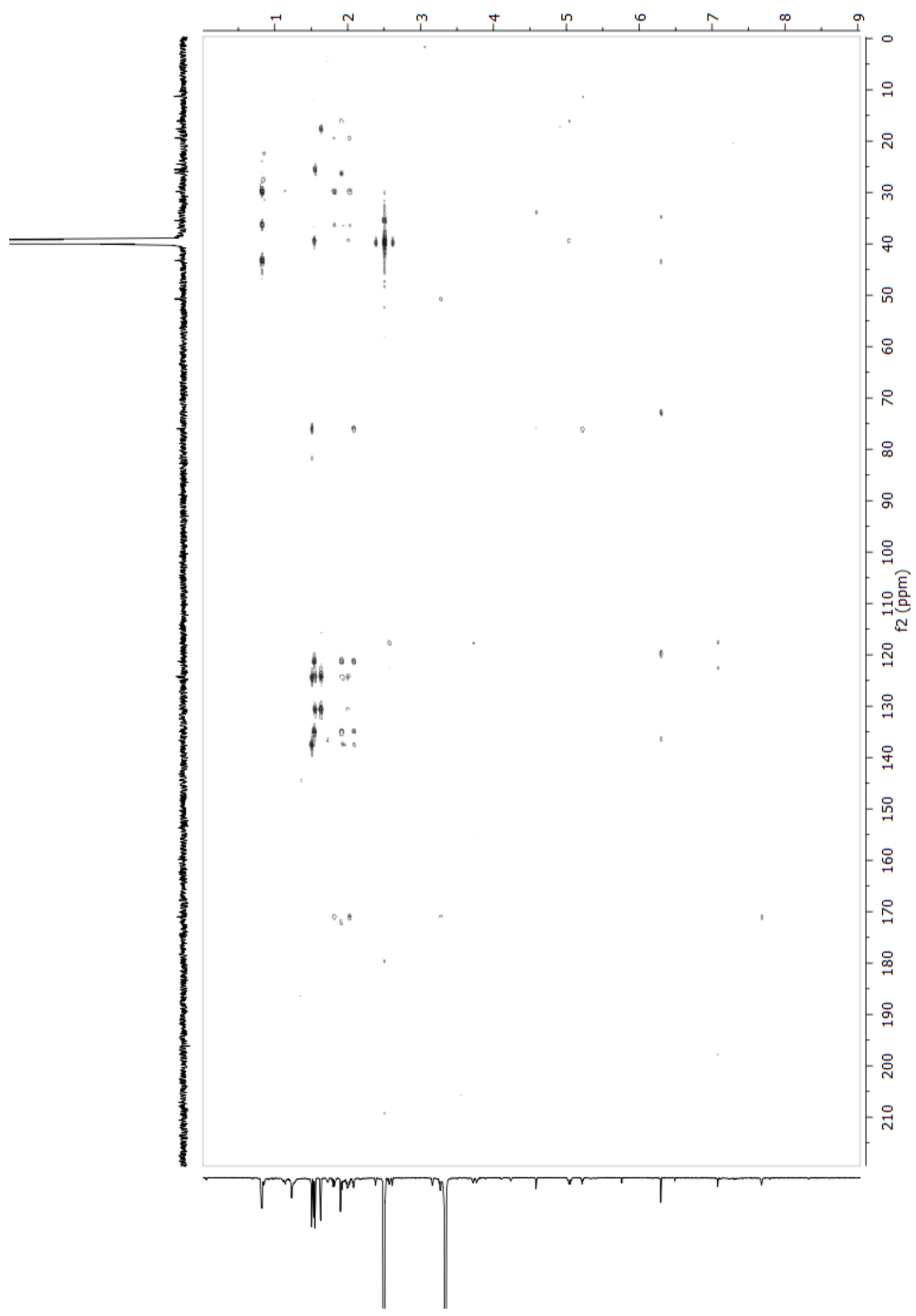

gHMBC NMR (DMSO-d 6 , $600 \mathrm{MHz}$, ) spectrum of rimarikiamide A (98). 


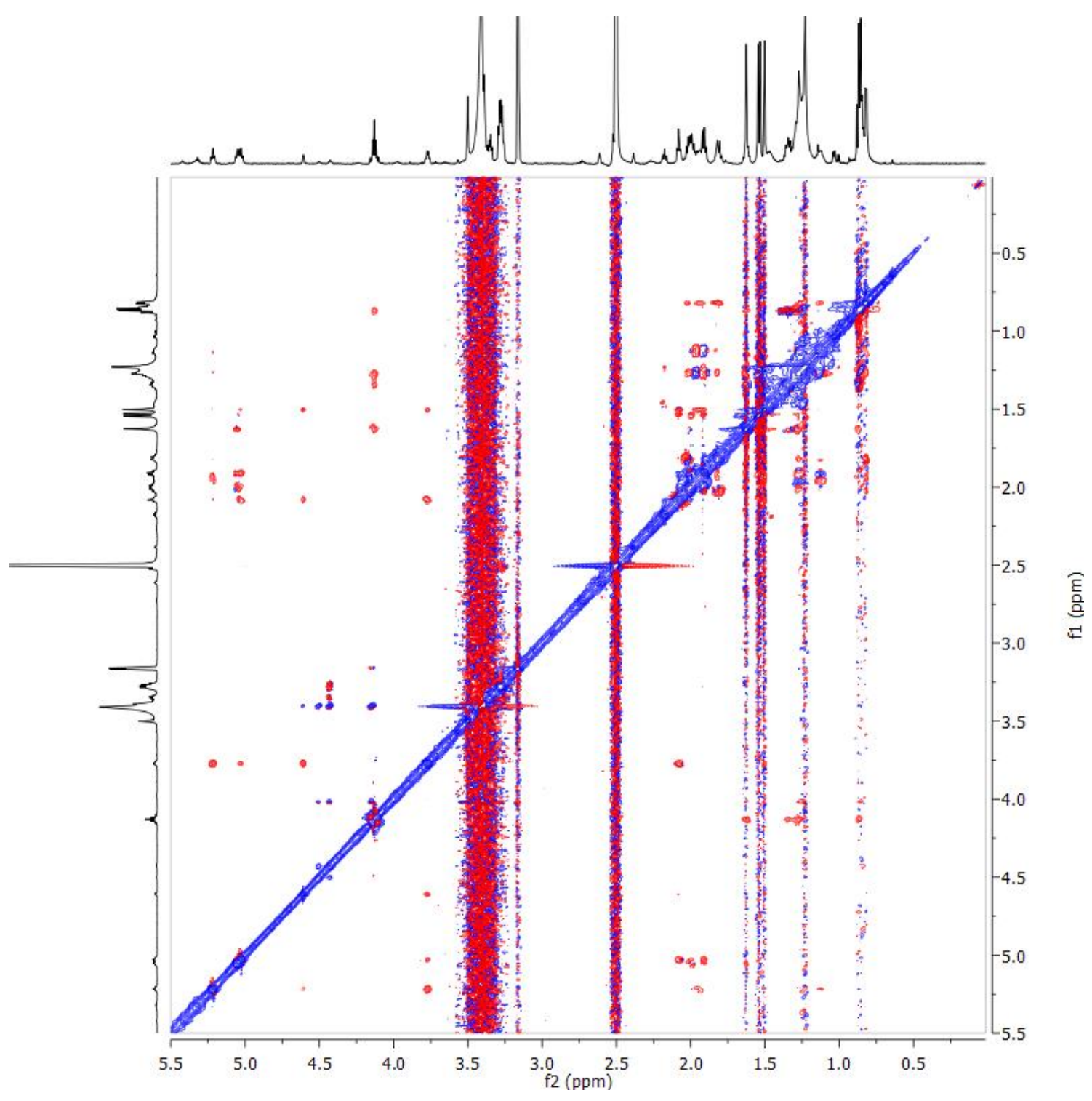

2D ROESY NMR (DMSO-d, $600 \mathrm{MHz}$, spectrum of rimarikiamide A (98). 


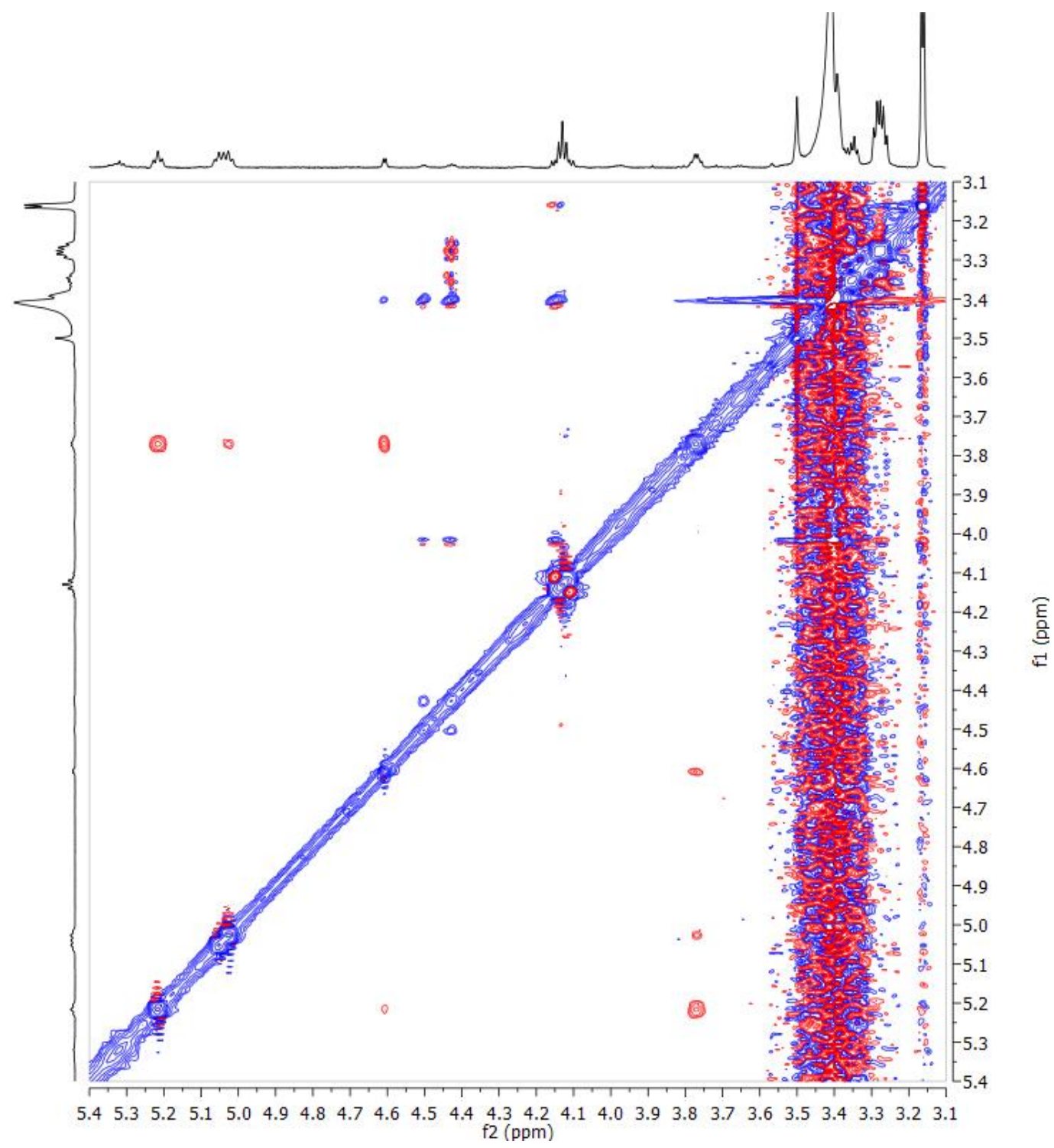

2D ROESY NMR (DMSO-d6, $600 \mathrm{MHz}$,) spectrum of rimarikiamide A (98) specific range of interest. 


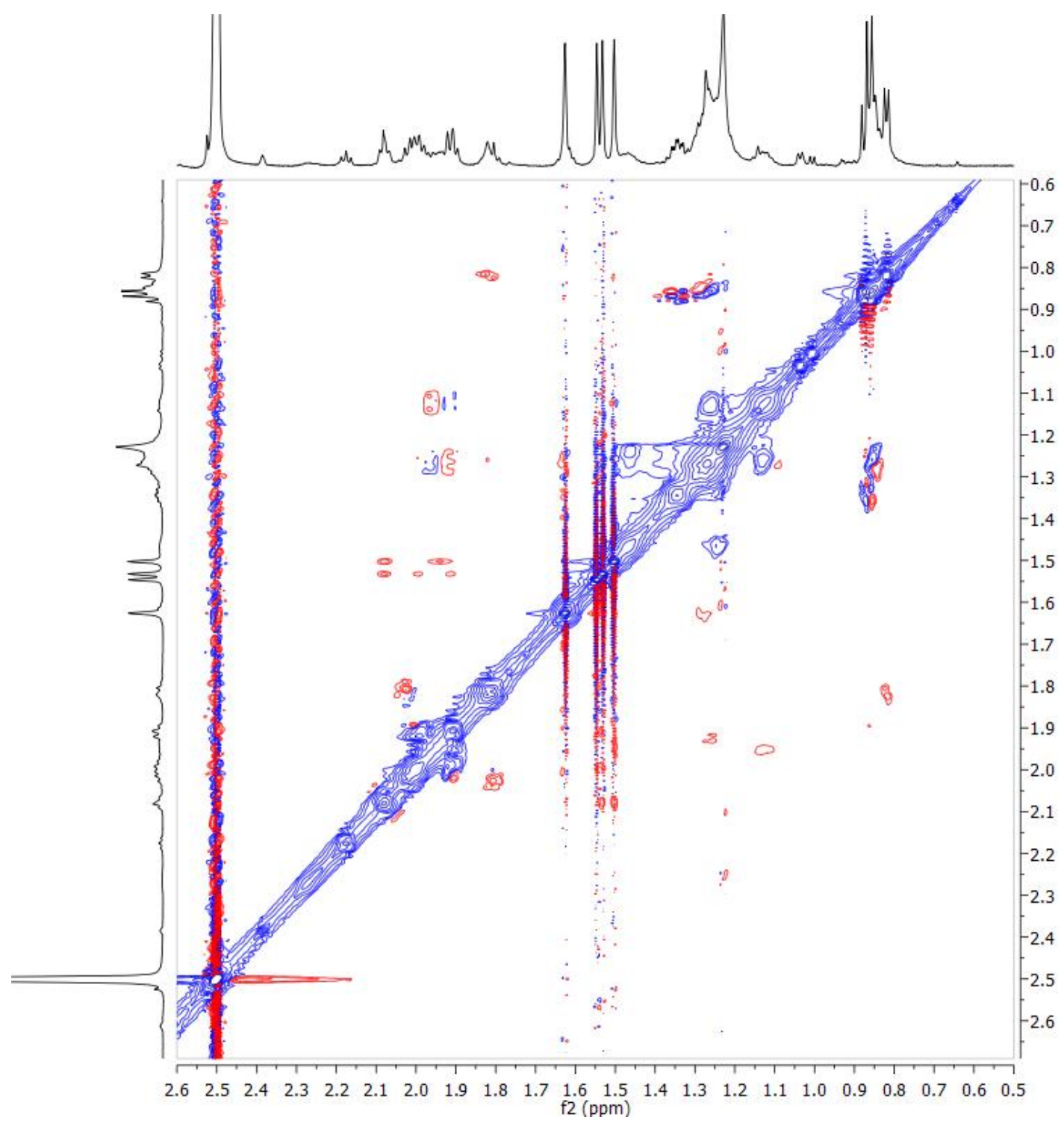

$\overparen{E}$
흠

2D ROESY NMR (DMSO-d 6 , $600 \mathrm{MHz}$, ) spectrum of rimarikiamide A (98) specific range of interest. 


\section{Appendix D: NMR spectra of 3-epi-xestoaminol C (156)}

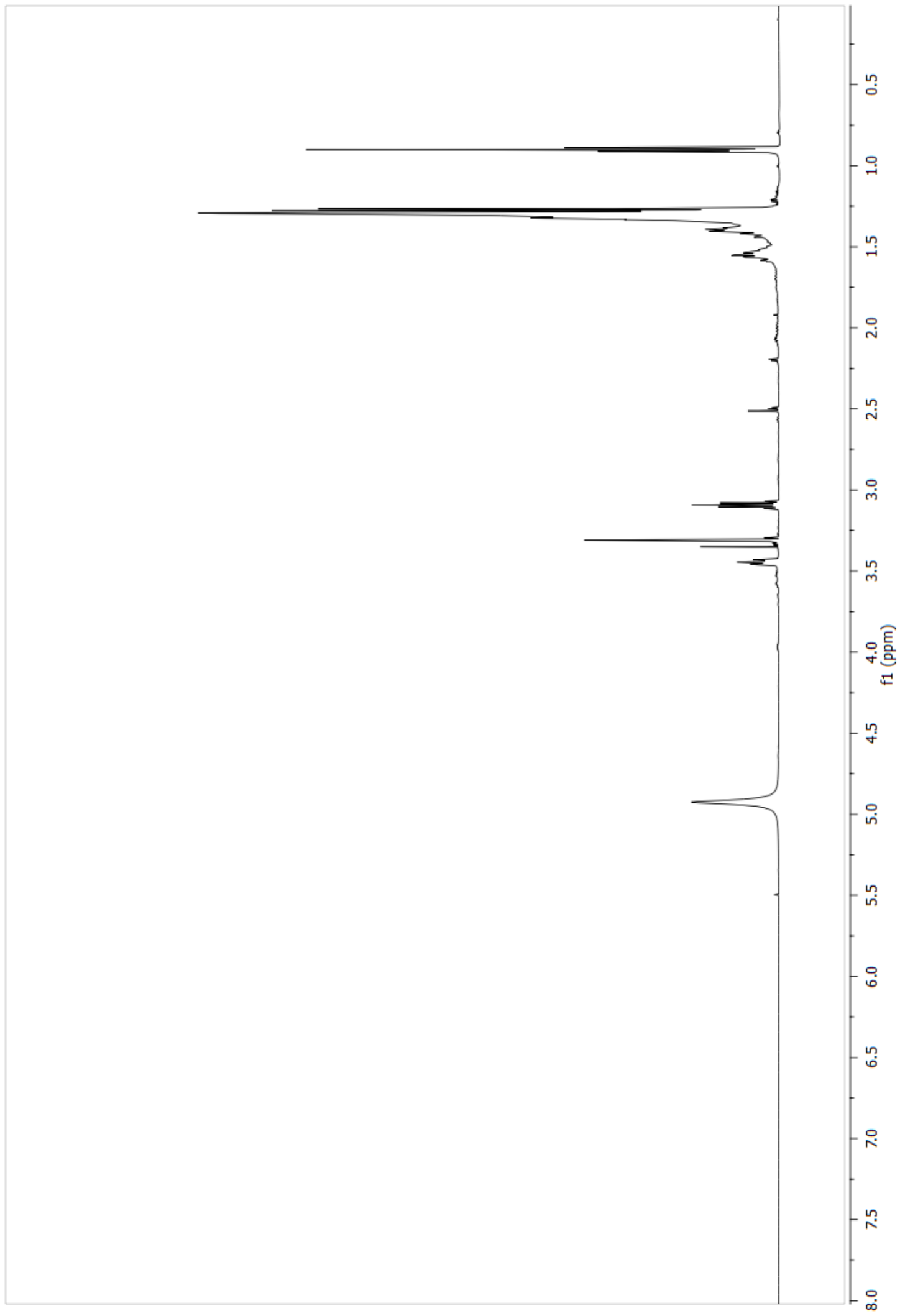

${ }^{1} \mathrm{H}$ NMR $\left(\mathrm{CD}_{3} \mathrm{OD}, 600 \mathrm{MHz}\right)$ spectrum of 3-epi-xestoaminol C (156). 


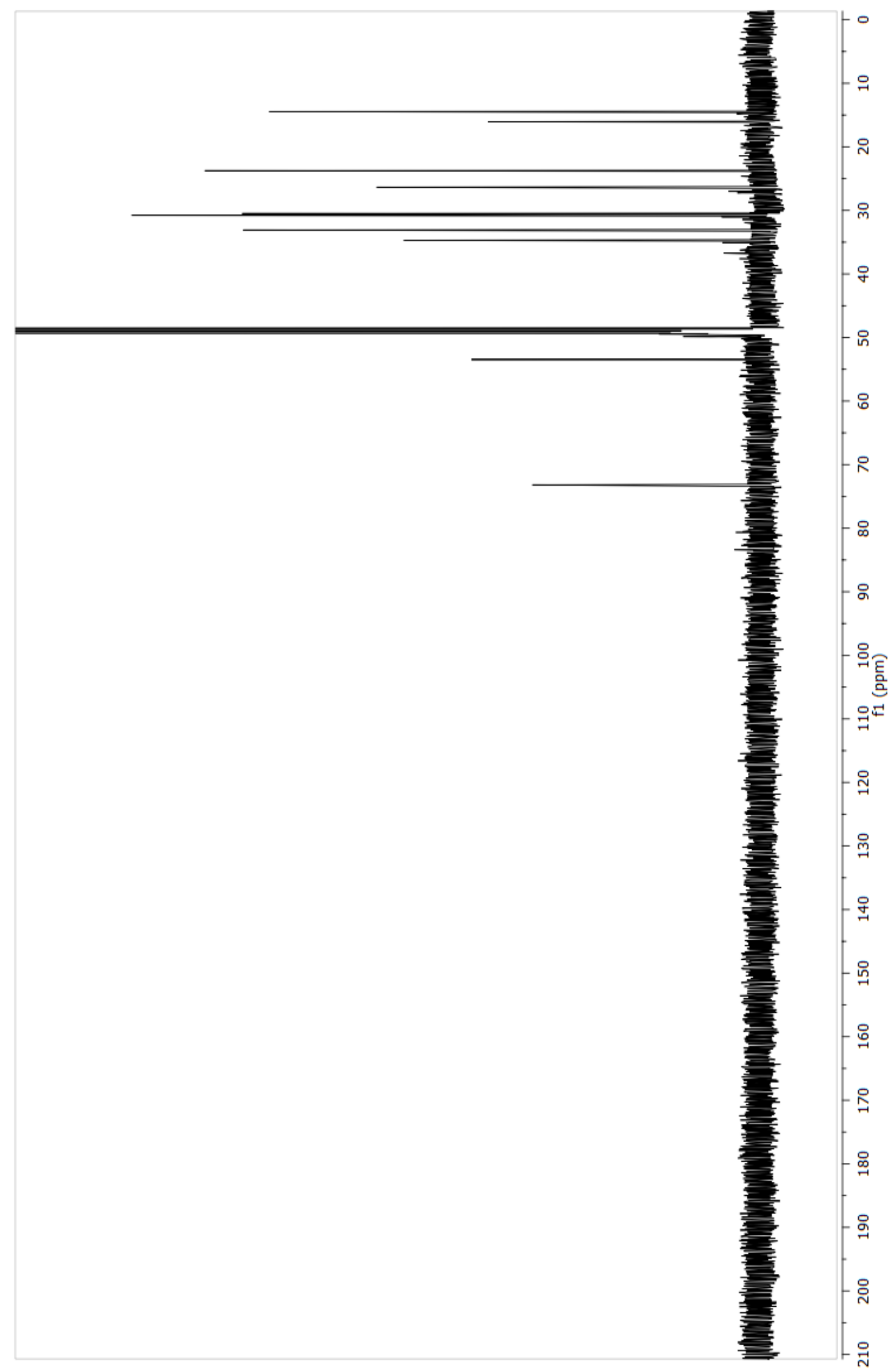

${ }^{13} \mathrm{C} \mathrm{NMR}\left(\mathrm{CD}_{3} \mathrm{OD}, 150 \mathrm{MHz}\right)$ spectrum of 3-epi-xestoaminol C (156). 


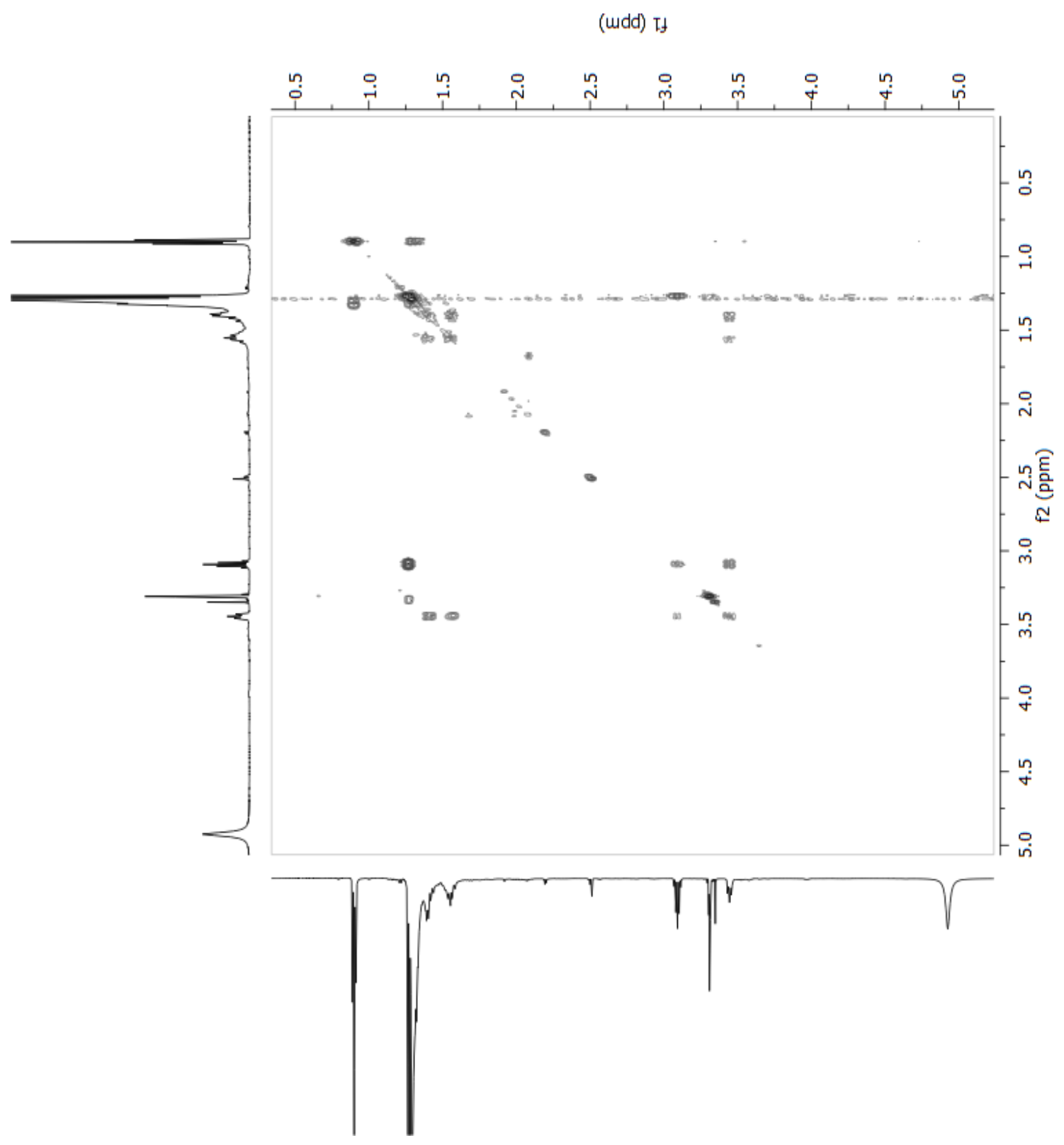

gCOSY NMR $\left(\mathrm{CD}_{3} \mathrm{OD}, 600 \mathrm{MHz}\right)$ spectrum of 3-epi-xestoaminol C (156). 
(udd) it

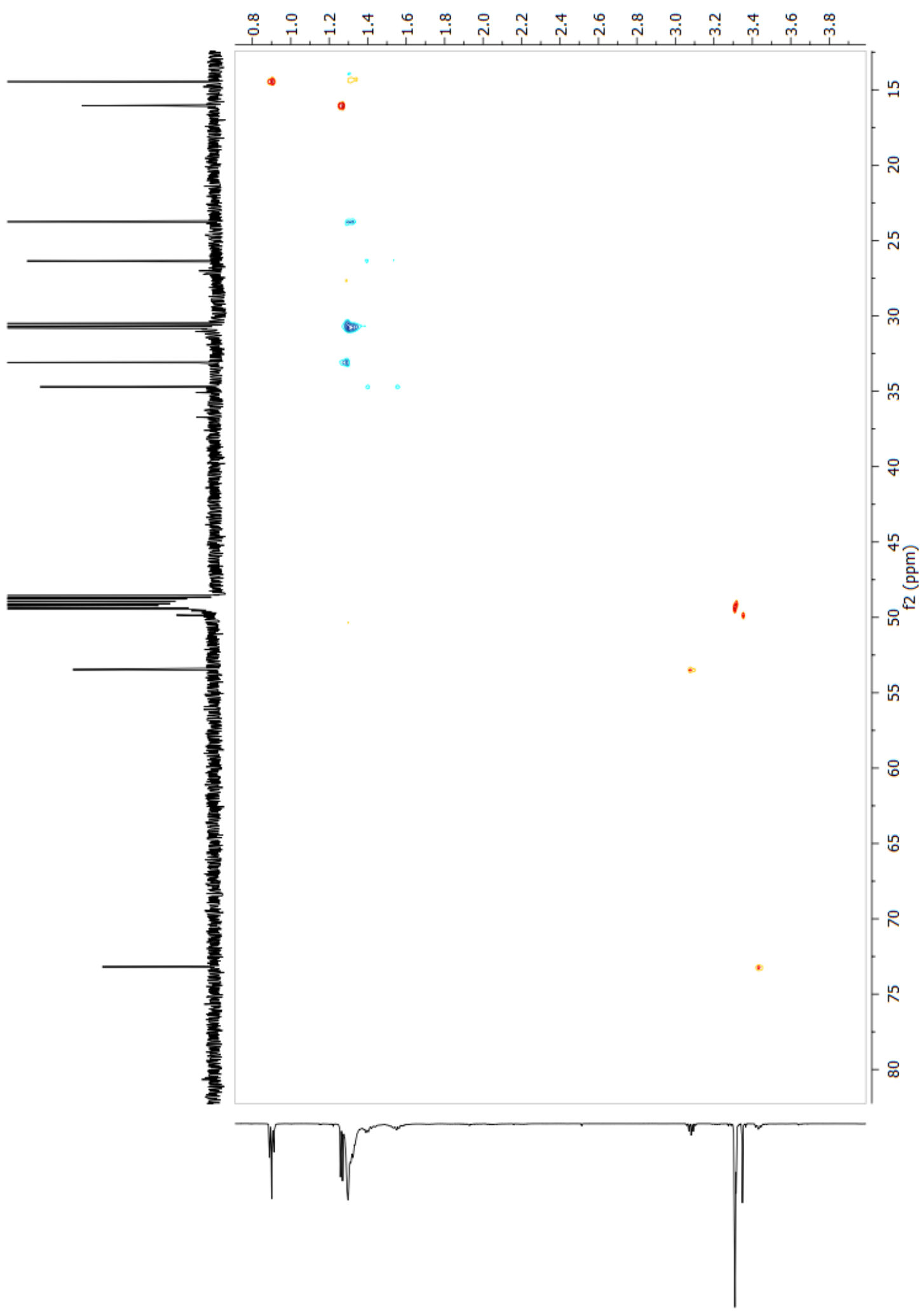

Multiplicity-edited HSQC NMR (CD $3 \mathrm{OD}, 600 \mathrm{MHz})$ spectrum of 3-epi-xestoaminol C (156) showing methylenes in blue and methyls and methines in red. 
(udd) if

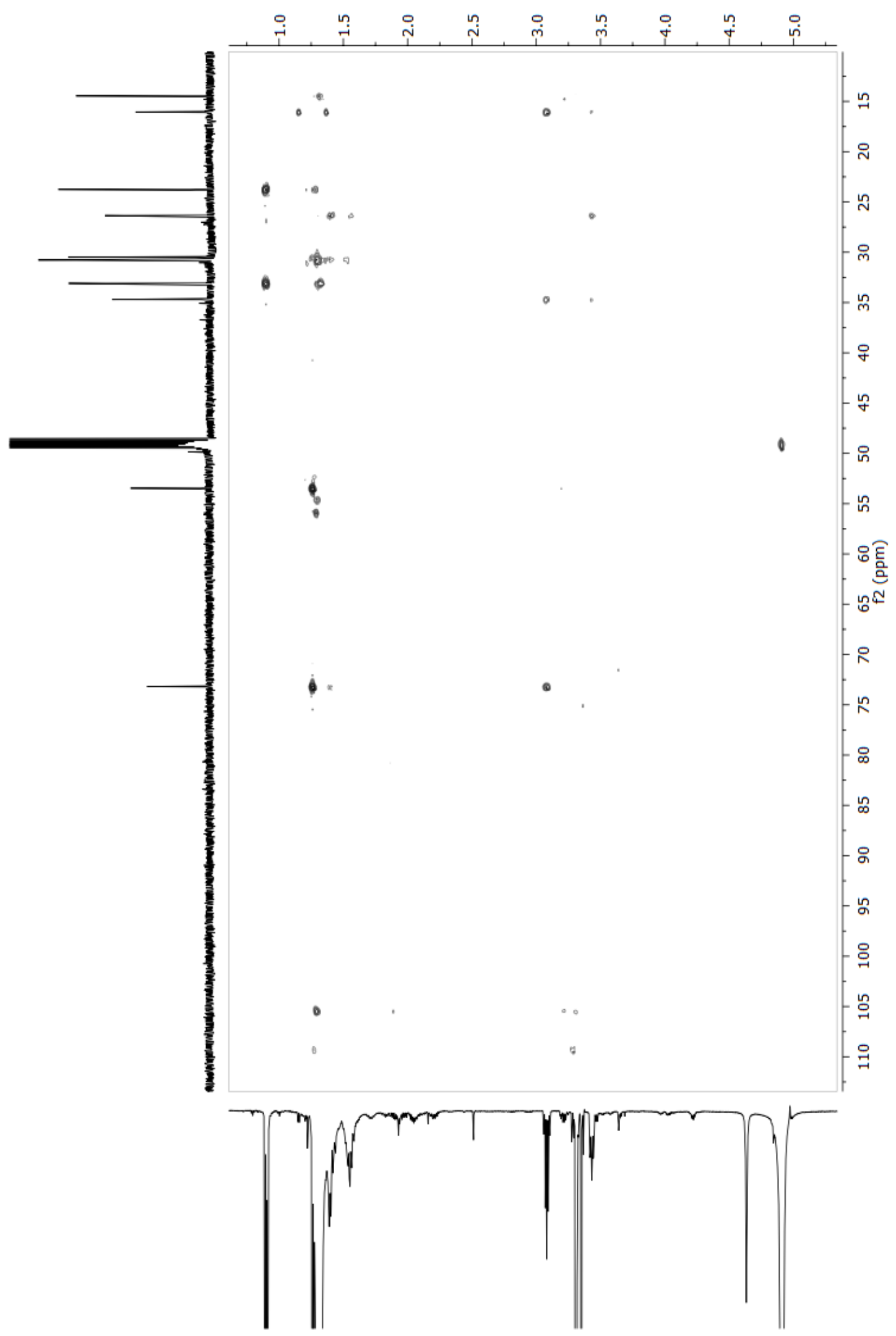

gHMBC NMR (CD 3 OD, $600 \mathrm{MHz})$ spectrum of 3-epi-xestoaminol C (156). 


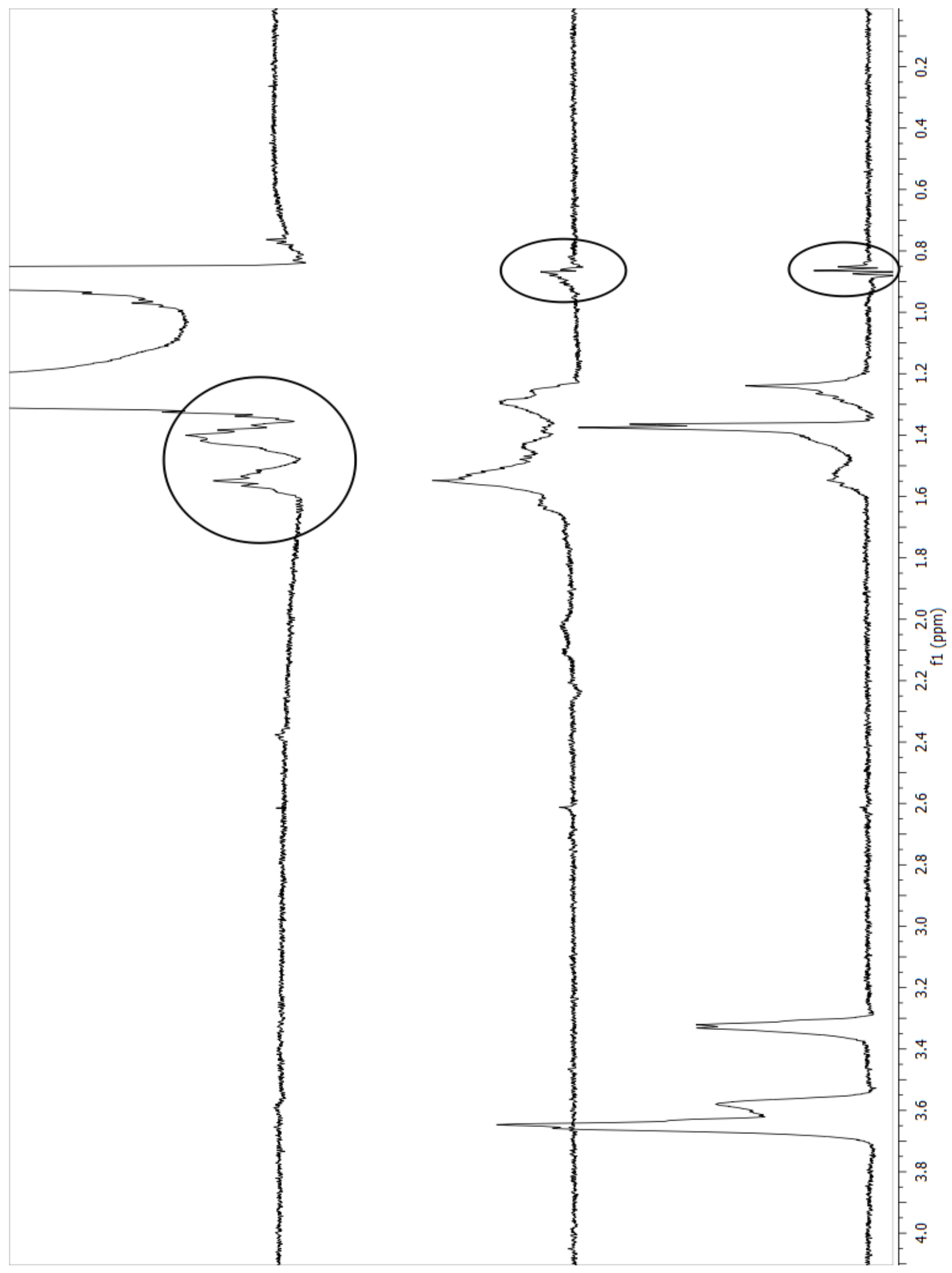

1D TOCSY NMR spectrum $\left(\mathrm{CDCl}_{3}, 600 \mathrm{MHz}, 200 \mathrm{~ms}\right.$ spin-lock mixing time) of 3-epixestoaminol C (156). Irradiation of $\mathrm{H}-3\left(\delta_{\mathrm{H}} 3.65\right)$ and $\mathrm{H}-4 / 5\left(\delta_{\mathrm{H}} 1.55\right)$ resulted in TOCSY transfer of energy to $\mathrm{H}_{3}-14\left(\delta_{\mathrm{H}} 0.88\right)$, indicated by circles in the bottom two panels. Irradiating $\mathrm{H}_{3}-14$ resulted in TOCSY transfer of energy to $\mathrm{H}-4 / 5$, indicated by a circle in the top panel. 


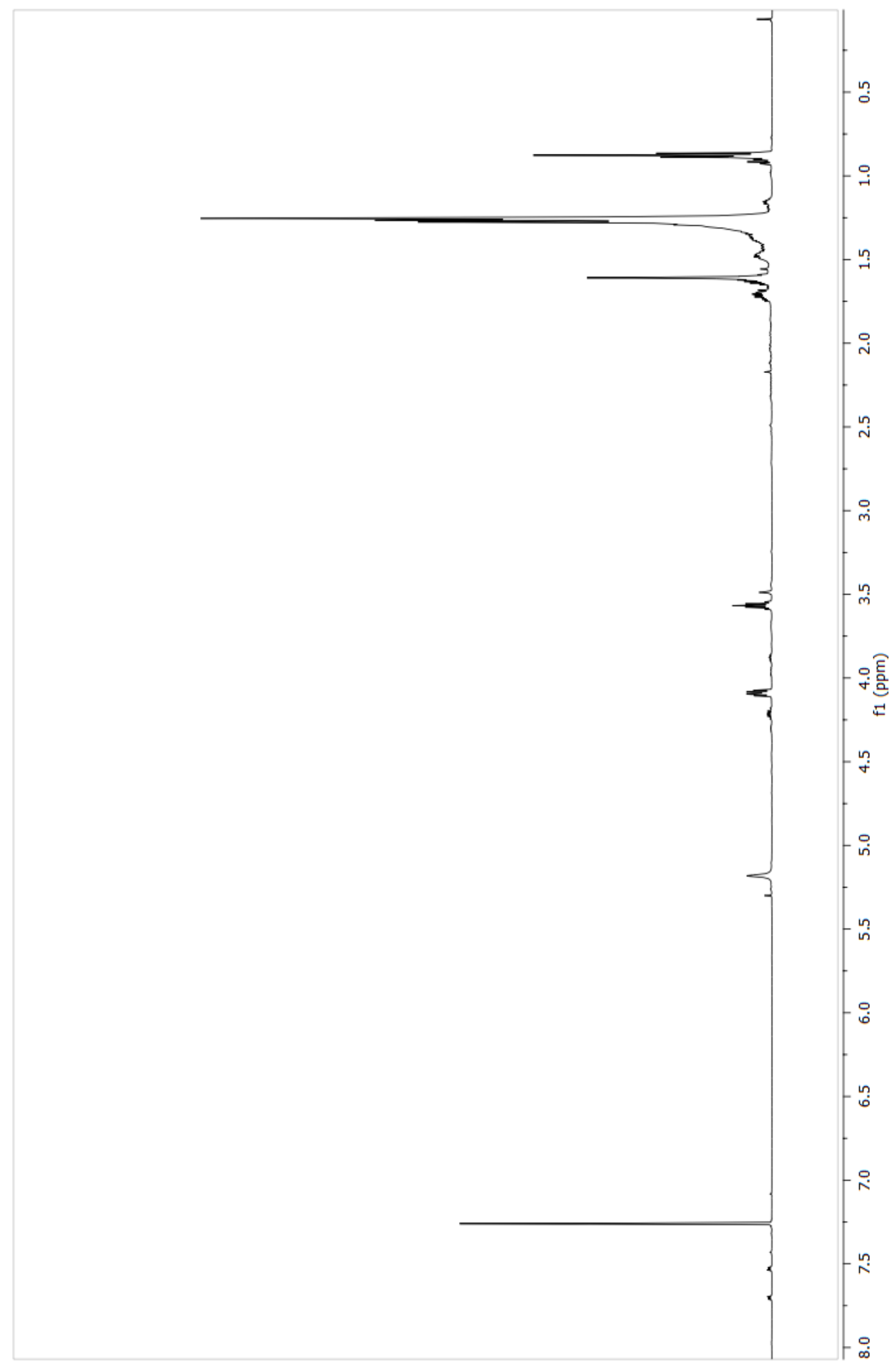

${ }^{1} \mathrm{H} \mathrm{NMR}\left(\mathrm{CDCl}_{3}, 600 \mathrm{MHz}\right)$ spectrum of oxazolidinone (157). 


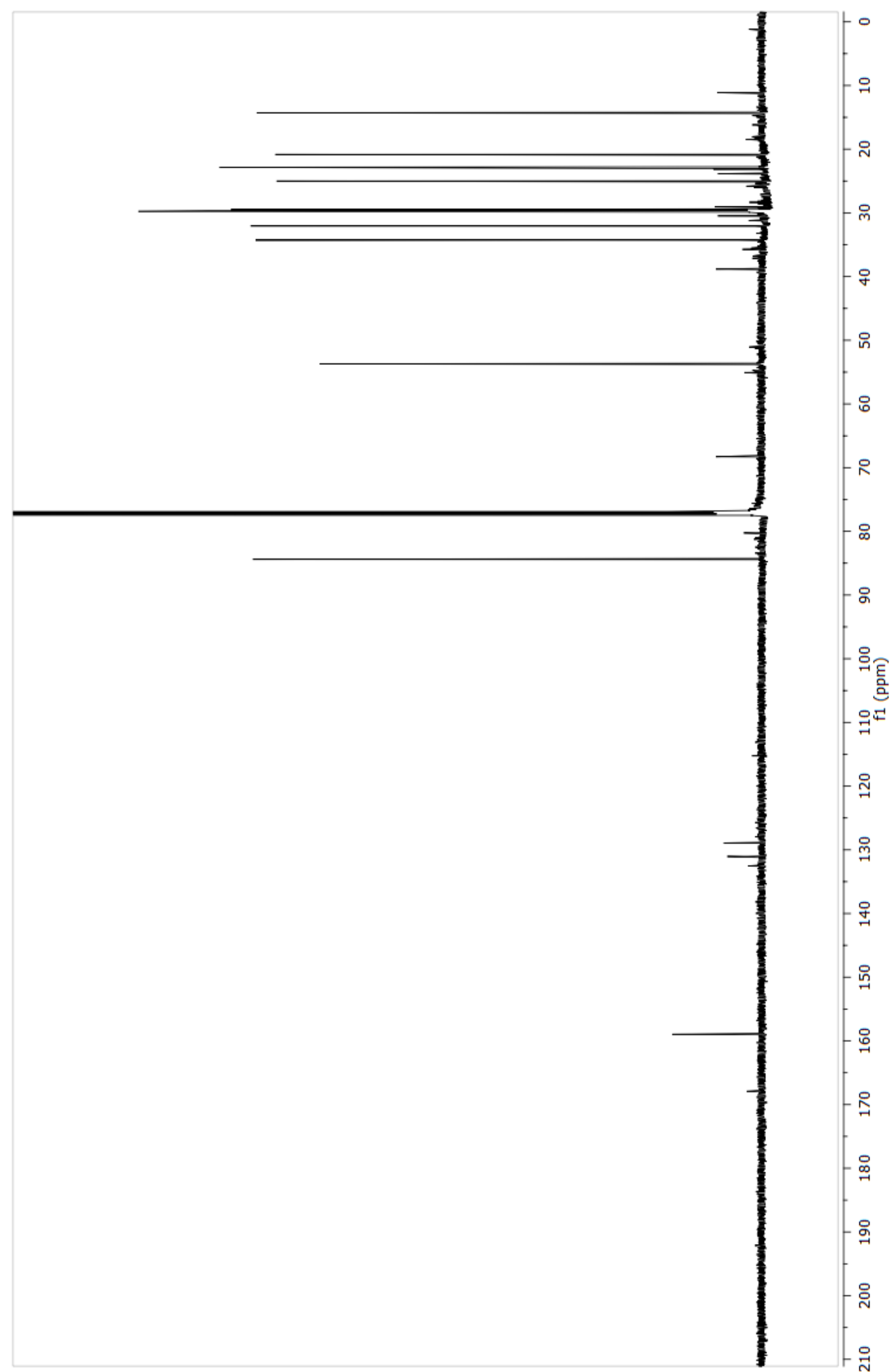

${ }^{13} \mathrm{C} \mathrm{NMR}\left(\mathrm{CDCl}_{3}, 150 \mathrm{MHz}\right)$ spectrum of oxazolidinone (157). 


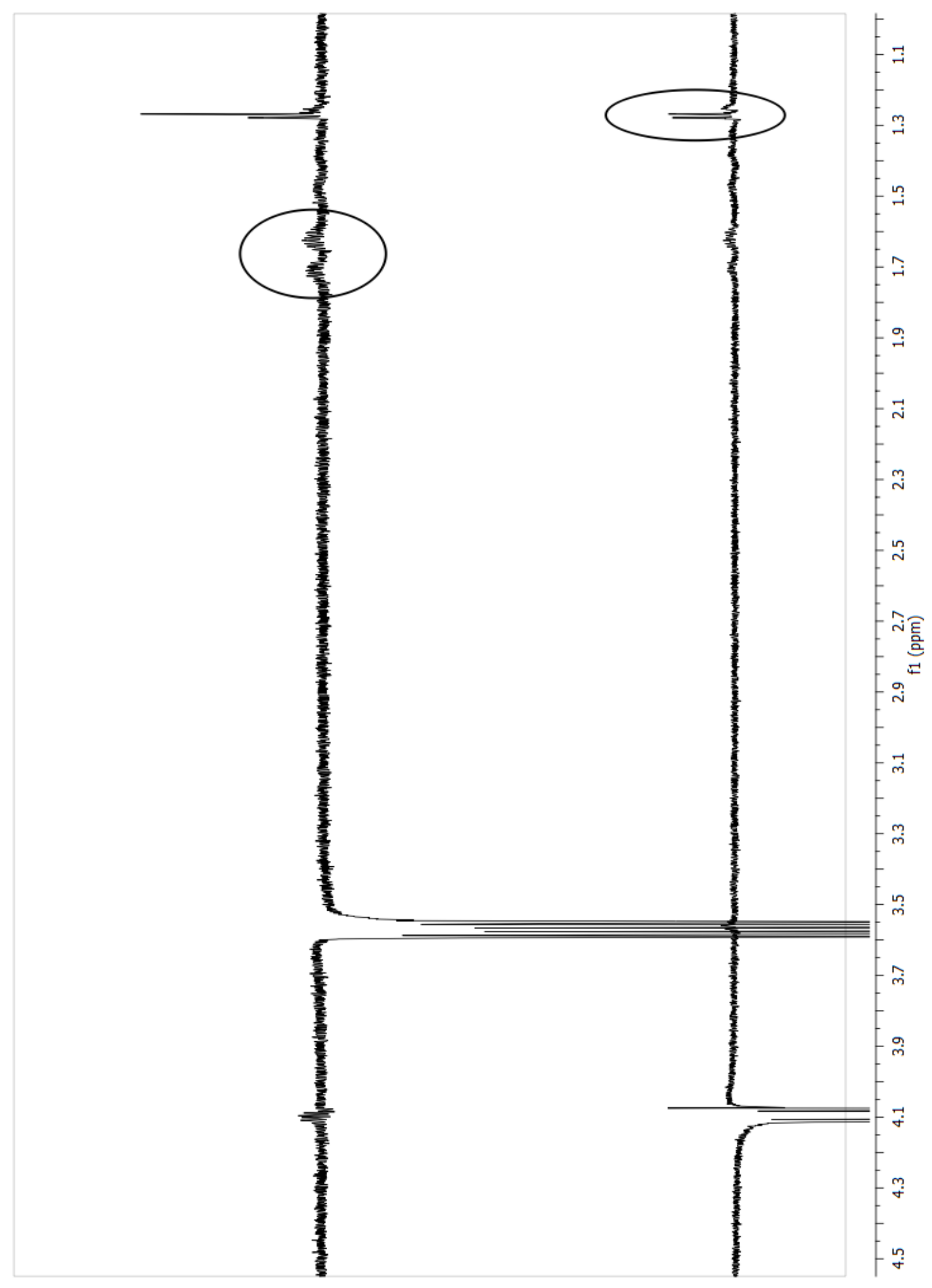

1D NOE NMR spectra $\left(\mathrm{CDCl}_{3}, 600 \mathrm{MHz}\right)$ spectrum of oxazolidinone (157). Irradiating $\mathrm{H}-2(\delta \mathrm{H} 3.57)$ resulted in NOE transferr to $\mathrm{H}-4 \mathrm{a}(\delta \mathrm{H} 1.62)$ and $\mathrm{H}-4 \mathrm{~b}(\delta \mathrm{H} 1.71)$ indicated by the circle in the top panel. Irradiating $\mathrm{H}-3(\delta \mathrm{H} 4.09)$ resulted in NOE transfer to $\mathrm{H} 3-1(\delta \mathrm{H} 1.27)$ indicated by the circle in the bottom panel. 


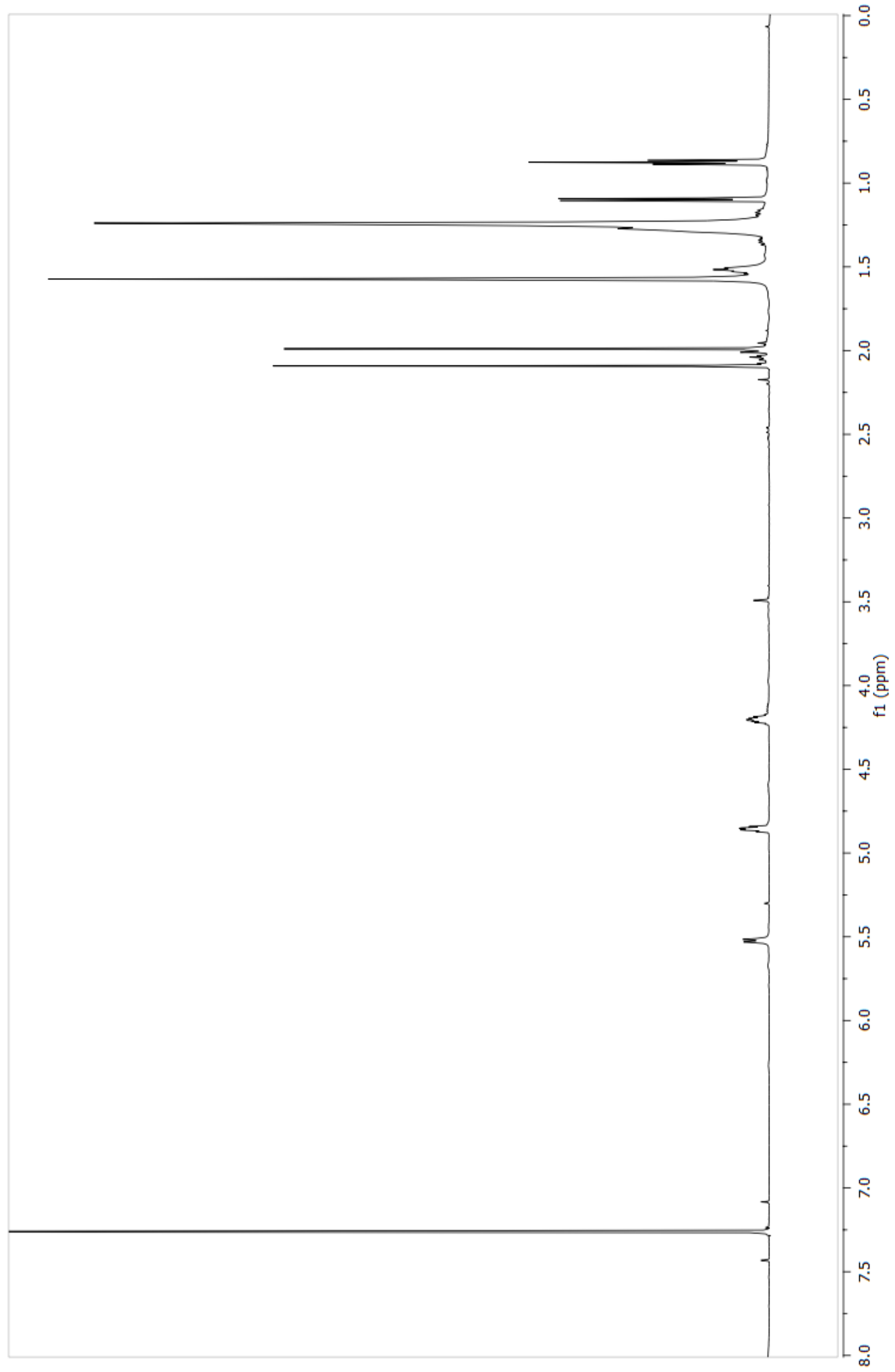

${ }^{1} \mathrm{H} \mathrm{NMR}\left(\mathrm{CDCl}_{3}, 600 \mathrm{MHz}\right)$ spectrum of diacetyl-3-epi-xestoaminol C (158). 


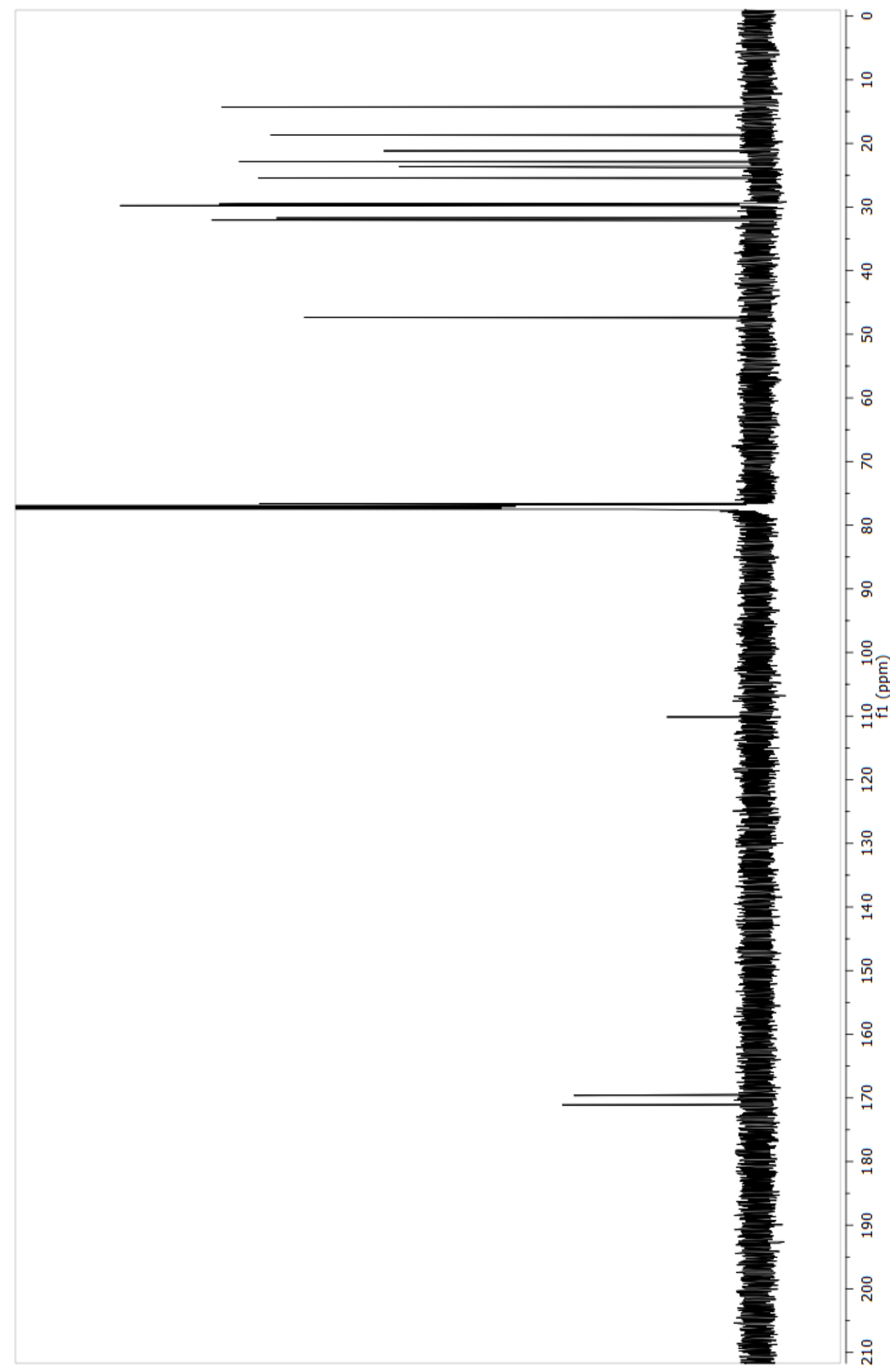

${ }^{13} \mathrm{C} \mathrm{NMR}\left(\mathrm{CDCl}_{3}, 150 \mathrm{MHz}\right)$ spectrum of diacetyl-3-epi-xestoaminol C (158). 


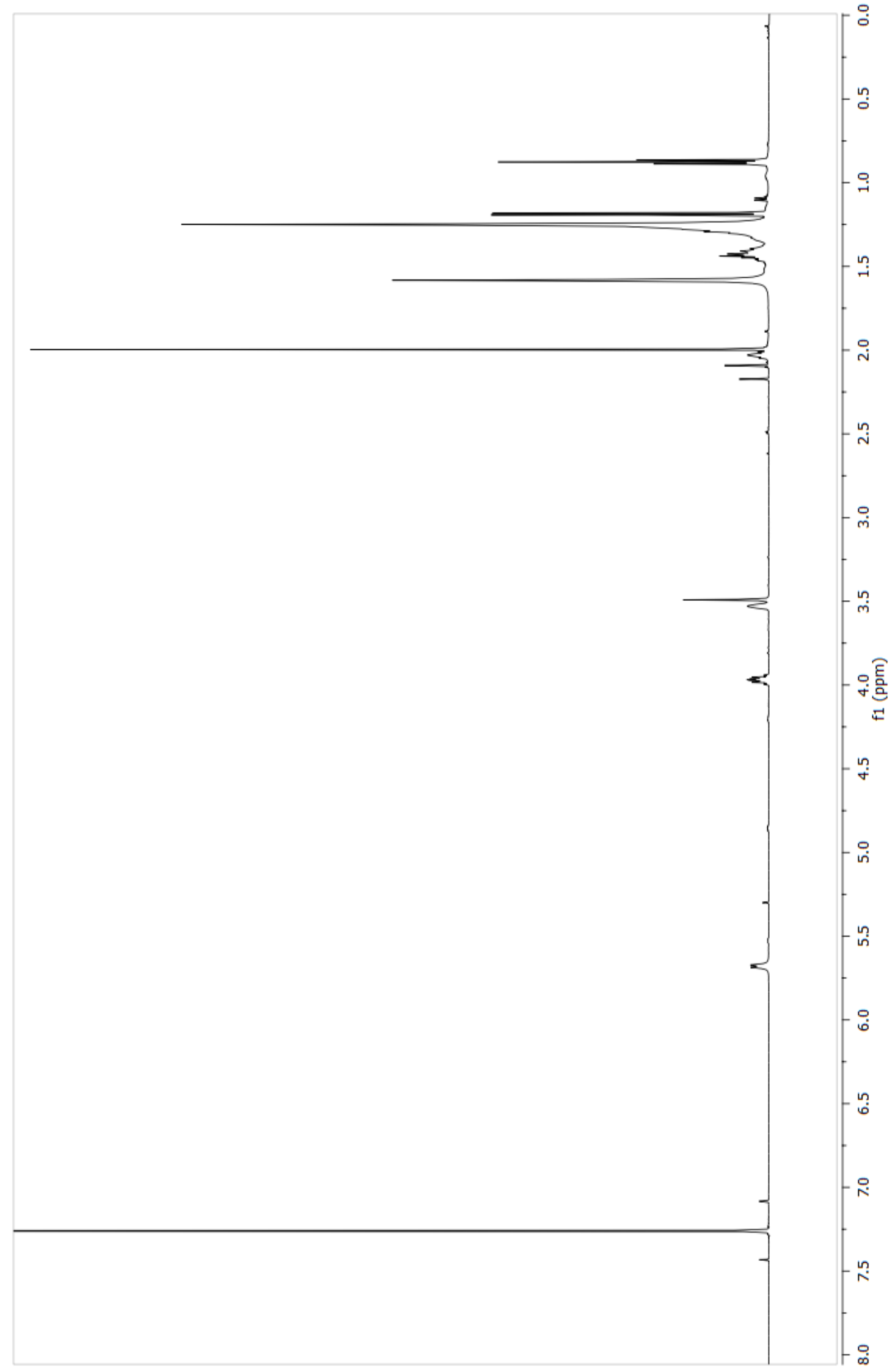

${ }^{1} \mathrm{H}$ NMR $\left(\mathrm{CDCl}_{3}, 600 \mathrm{MHz}\right)$ spectrum of $N$-acetyl-3-epi-xestoaminol C (159). 


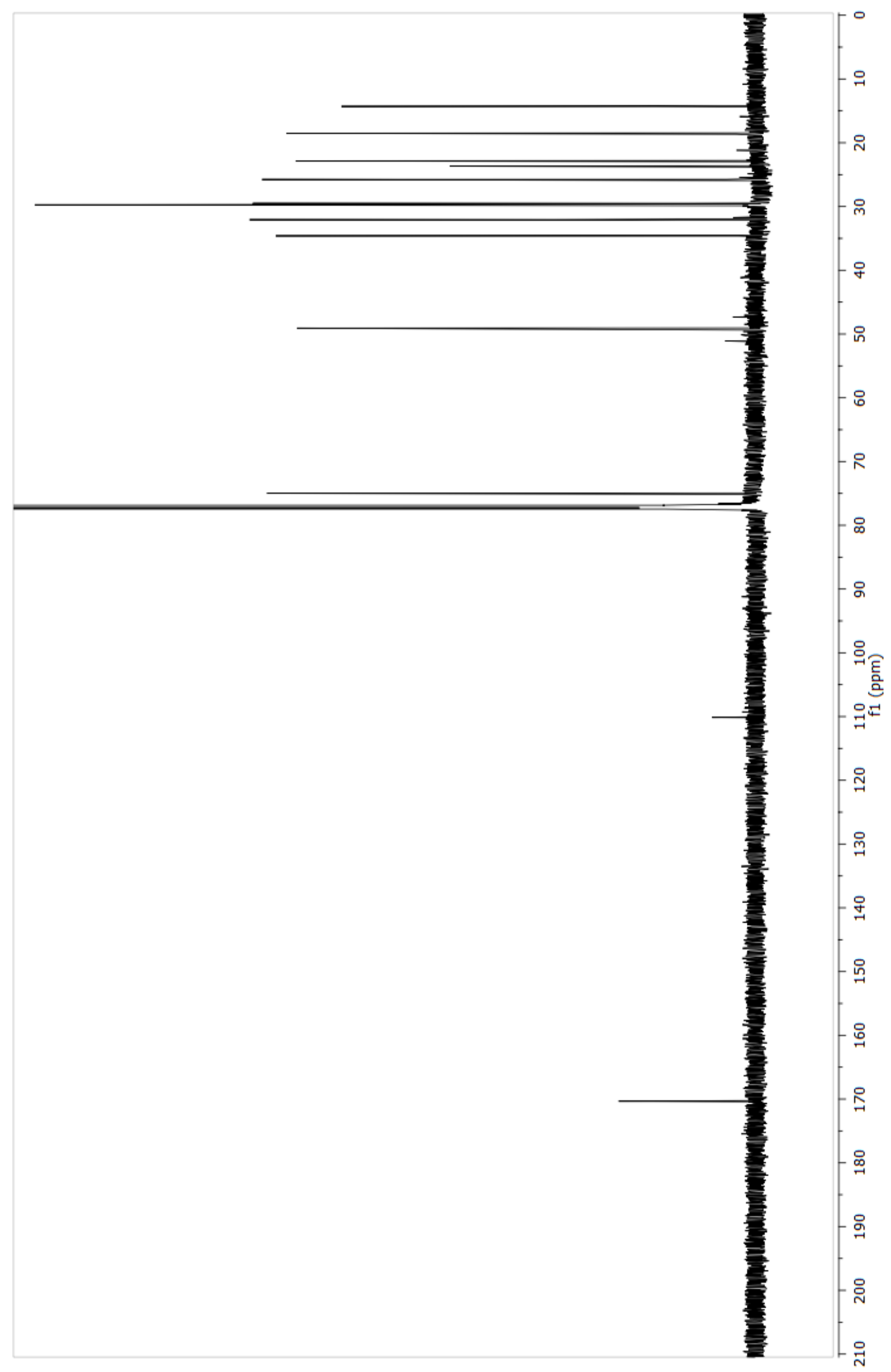

${ }^{13} \mathrm{C} \mathrm{NMR}\left(\mathrm{CDCl}_{3}, 150 \mathrm{MHz}\right)$ spectrum of $\mathrm{N}$-acetyl-3-epi-xestoaminol C (159). 


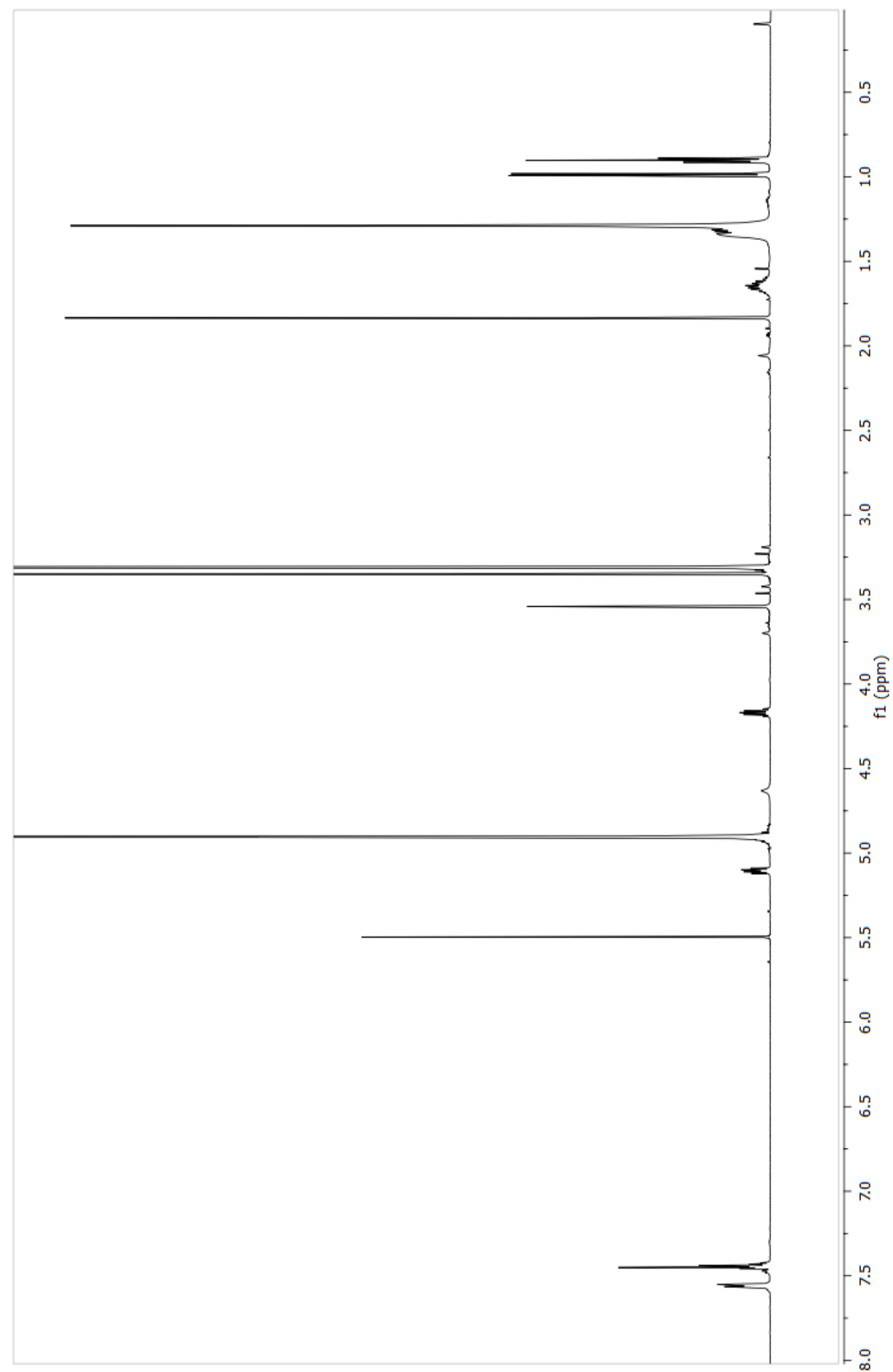

${ }^{1} \mathrm{H}$ NMR $\left(\mathrm{CD}_{3} \mathrm{OD}, 600 \mathrm{MHz}\right)$ spectrum of the $R$ - Mosher's ester (160). 


$$
1
$$




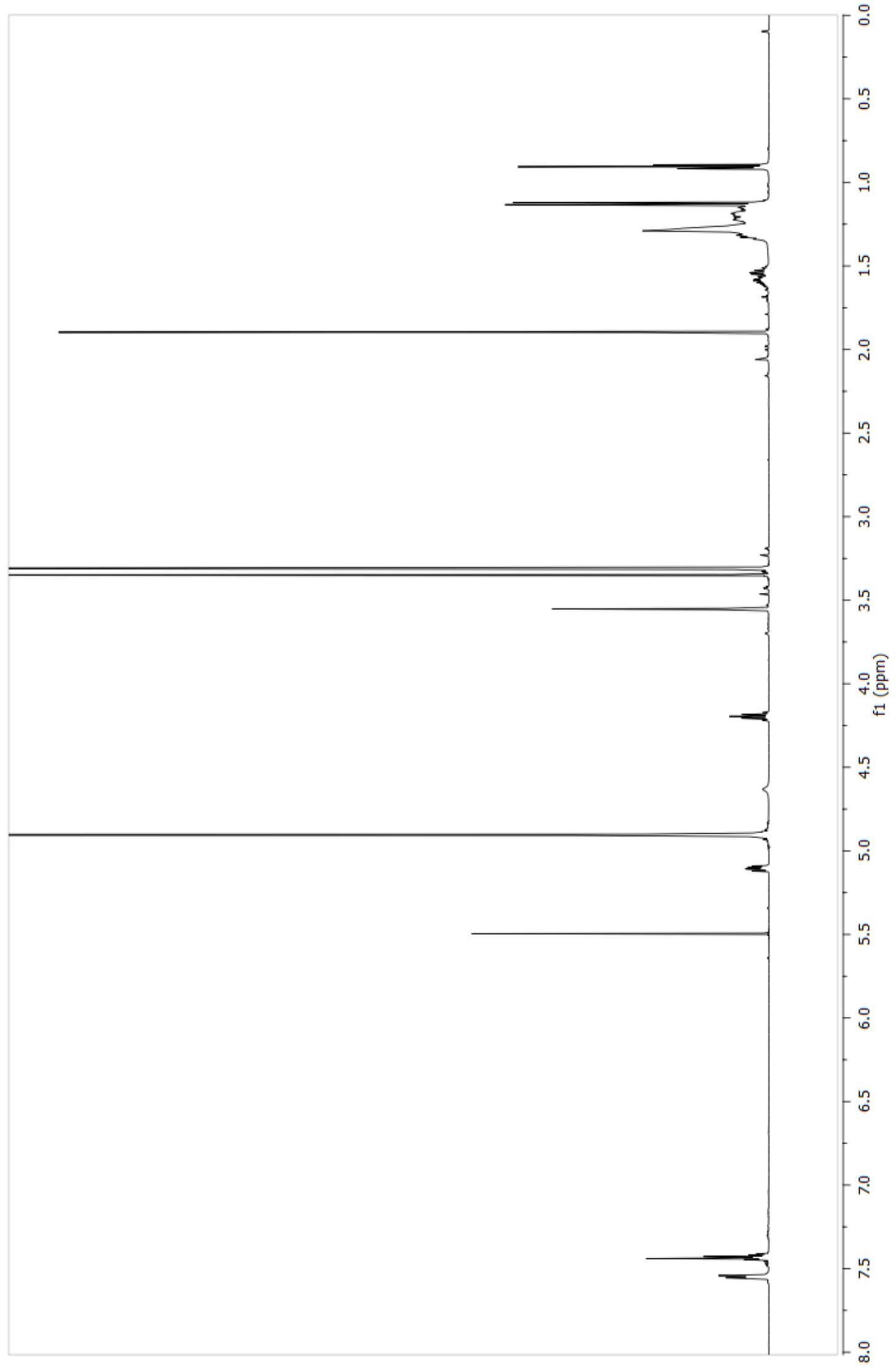

${ }^{1} \mathrm{H}$ NMR $\left(\mathrm{CD}_{3} \mathrm{OD}, 600 \mathrm{MHz}\right)$ spectrum of the $S$ - Mosher's ester (161). 


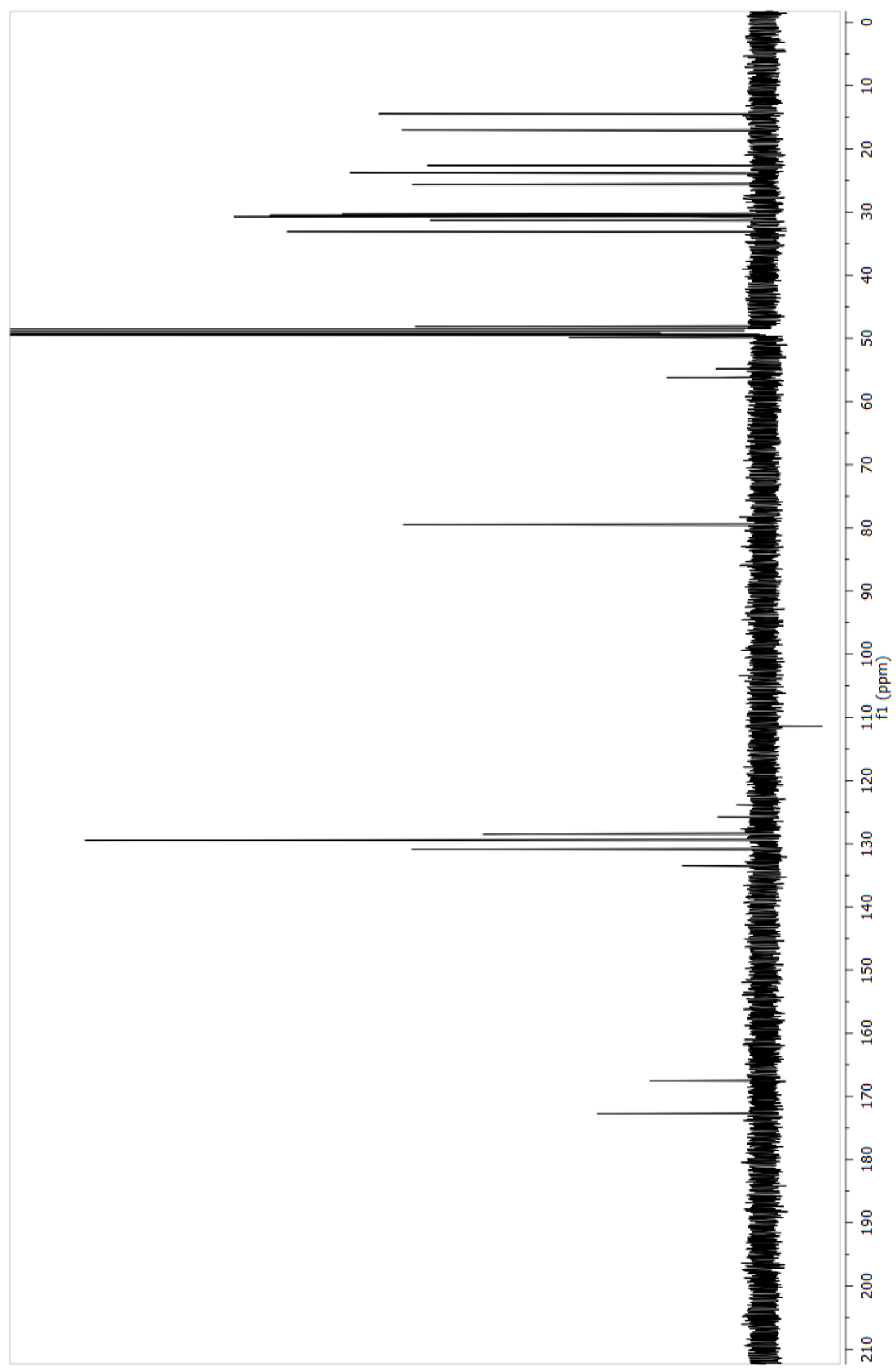

${ }^{13} \mathrm{C}$ NMR $\left(\mathrm{CD}_{3} \mathrm{OD}, 150 \mathrm{MHz}\right)$ spectrum of the $S$-Mosher's ester (161). 


\section{Appendix E: Chemical genetics mutant hits}

Top 28 S. cerevisiae mutants identifed for chemcial genetic profiling.

\begin{tabular}{|c|c|c|c|c|}
\hline $\begin{array}{l}\text { Residual } \\
\text { Growth }\end{array}$ & $P$-values & ORF & Gene Name & Description \\
\hline 64.9 & 0.000 & YNL202W & SPS19 & $\begin{array}{l}\text { Peroxisomal 2,4-dienoyl-CoA reductase; auxiliary } \\
\text { enzyme of fatty acid beta-oxidation; homodimeric } \\
\text { enzyme required for growth and sporulation on } \\
\text { petroselineate medium; expression induced during } \\
\text { late sporulation and in the presence of oleate }\end{array}$ \\
\hline 57.0 & 0.003 & YOL011W & PLB3 & $\begin{array}{l}\text { Phospholipase B (lysophospholipase) involved in } \\
\text { lipid metabolism; hydrolyzes phosphatidylinositol and } \\
\text { phosphatidylserine and displays transacylase activity } \\
\text { in vitro; PLB3 has a paralog, PLB1, that arose from } \\
\text { the whole genome duplication }\end{array}$ \\
\hline 40.6 & 0.004 & YJL216C & IMA5 & $\begin{array}{l}\text { Alpha-glucosidase; specificity for isomaltose, } \\
\text { maltose, and palatinose, but not alpha- } \\
\text { methylglucoside; member of the IMA isomaltase } \\
\text { family; not required for isomaltose utilization, but } \\
\text { Ima5p overexpression allows the imal null mutant to } \\
\text { grow on isomaltose }\end{array}$ \\
\hline 60.5 & 0.004 & YDR032C & PST2 & $\begin{array}{l}\text { Protein with similarity to a family of flavodoxin-like } \\
\text { proteins; induced by oxidative stress in a Yap1p } \\
\text { dependent manner; the authentic, non-tagged protein } \\
\text { is detected in highly purified mitochondria in high- } \\
\text { throughput studies; protein abundance increases in } \\
\text { response to DNA replication stress; PST2 has a } \\
\text { paralog, RFS1, that arose from the whole genome } \\
\text { duplication }\end{array}$ \\
\hline 43.2 & 0.004 & YNL090W & RHO2 & $\begin{array}{l}\text { Non-essential small GTPase of the Rho/Rac family of } \\
\text { Ras-like proteins; involved in the establishment of } \\
\text { cell polarity and in microtubule assembly }\end{array}$ \\
\hline 64.8 & 0.005 & YDR257C & RKM4 & $\begin{array}{l}\text { Ribosomal lysine methyltransferase; specific for } \\
\text { monomethylation of Rpl42ap and Rpl42bp (lysine } \\
\text { 55); nuclear SET-domain containing protein }\end{array}$ \\
\hline 57.4 & 0.006 & YDR193W & YDR193W & $\begin{array}{l}\text { Dubious open reading frame; unlikely to encode a } \\
\text { functional protein, based on available experimental } \\
\text { and comparative sequence data }\end{array}$ \\
\hline 45.3 & 0.006 & YDR183W & PLP1 & $\begin{array}{l}\text { Protein that interacts with CCT (chaperonin } \\
\text { containing TCP-1) complex; has a role in actin and } \\
\text { tubulin folding; has weak similarity to phosducins, } \\
\text { which are G-protein regulators }\end{array}$ \\
\hline 62.65 & 0.007 & $\begin{array}{l}\text { YLR361C- } \\
\text { A }\end{array}$ & YLR361C-A & Putative protein of unknown function \\
\hline 46.8 & 0.008 & YBR059C & AKL1 & $\begin{array}{l}\text { Ser-Thr protein kinase; member (with Ark1p and } \\
\text { Prk1p) of the Ark kinase family; involved in } \\
\text { endocytosis and actin cytoskeleton organization }\end{array}$ \\
\hline 61.1 & 0.013 & YDR272W & GLO2 & $\begin{array}{l}\text { Cytoplasmic glyoxalase II; catalyzes the hydrolysis of } \\
\text { S-D-lactoylglutathione into glutathione and D-lactate; } \\
\text { GLO2 has a paralog, GLO4, that arose from the } \\
\text { whole genome duplication }\end{array}$ \\
\hline 58.5 & 0.013 & YNL235C & YNL235C & $\begin{array}{l}\text { Dubious open reading frame; unlikely to encode a } \\
\text { functional protein, based on available experimental } \\
\text { and comparative sequence data; partially overlaps the } \\
\text { verified ORF SIN4/YNL236W, a subunit of the } \\
\text { mediator complex }\end{array}$ \\
\hline 69.4 & 0.014 & YBL007C & SLA1 & $\begin{array}{l}\text { Cytoskeletal protein binding protein; required for } \\
\text { assembly of the cortical actin cytoskeleton; interacts } \\
\text { with proteins regulating actin dynamics and proteins } \\
\text { required for endocytosis; found in the nucleus and cell } \\
\text { cortex; has } 3 \text { SH3 domains }\end{array}$ \\
\hline 43.8 & 0.016 & YIL010W & DOT5 & $\begin{array}{l}\text { Nuclear thiol peroxidase; functions as an alkyl- } \\
\text { hydroperoxide reductase during post-diauxic growth }\end{array}$ \\
\hline
\end{tabular}




\begin{tabular}{|c|c|c|c|c|}
\hline 61.5 & 0.017 & YDR183W & PLP1 & $\begin{array}{l}\text { Protein that interacts with CCT (chaperonin } \\
\text { containing TCP-1) complex; has a role in actin and } \\
\text { tubulin folding; has weak similarity to phosducins, } \\
\text { which are G-protein regulators }\end{array}$ \\
\hline 63.6 & 0.019 & YBL008W & HIR1 & $\begin{array}{l}\text { Subunit of the HIR complex; HIR is a nucleosome } \\
\text { assembly complex involved in regulation of histone } \\
\text { gene transcription; contributes to nucleosome } \\
\text { formation, heterochromatic gene silencing, and } \\
\text { formation of functional kinetochores }\end{array}$ \\
\hline 69.1 & 0.019 & YBR276C & PPS1 & $\begin{array}{l}\text { Protein phosphatase; has specificity for serine, } \\
\text { threonine, and tyrosine residues; has a role in the } \\
\text { DNA synthesis phase of the cell cycle }\end{array}$ \\
\hline 61.3 & 0.025 & $\begin{array}{c}\text { YAL064C- } \\
\text { A }\end{array}$ & TDA8 & $\begin{array}{l}\text { Putative protein of unknown function; null mutant is } \\
\text { sensitive to expression of the top1-T722A allele; not } \\
\text { an essential gene }\end{array}$ \\
\hline 62.9 & 0.026 & YDL237W & AIM6 & $\begin{array}{l}\text { Putative protein of unknown function; required for } \\
\text { respiratory growth; YDL } 237 \mathrm{~W} \text { is not an essential } \\
\text { gene }\end{array}$ \\
\hline 66.5 & 0.029 & YJL191W & RPS14B & $\begin{array}{l}\text { Protein component of the small (40S) ribosomal } \\
\text { subunit; required for ribosome assembly and } 20 \mathrm{~S} \text { pre- } \\
\text { rRNA processing; mutations confer cryptopleurine } \\
\text { resistance; homologous to mammalian ribosomal } \\
\text { protein S14 and bacterial S11; RPS14B has a paralog, } \\
\text { RPS14A, that arose from the whole genome } \\
\text { duplication }\end{array}$ \\
\hline 35.3 & 0.029 & YHL020C & OPI1 & $\begin{array}{l}\text { Transcriptional regulator of a variety of genes; } \\
\text { phosphorylation by protein kinase A stimulates Opilp } \\
\text { function in negative regulation of phospholipid } \\
\text { biosynthetic genes; involved in telomere maintenance; } \\
\text { null exhibits disrupted mitochondrial metabolism and } \\
\text { low cardiolipin content, strongly correlated with } \\
\text { overproduction of inositol }\end{array}$ \\
\hline 61.8 & 0.030 & YBL017C & PEP1 & $\begin{array}{l}\text { Type I transmembrane sorting receptor for multiple } \\
\text { vacuolar hydrolases; cycles between the late-Golgi } \\
\text { and prevacuolar endosome-like compartments }\end{array}$ \\
\hline 65.6 & 0.031 & YOR300W & YOR300W & $\begin{array}{l}\text { Dubious open reading frame; unlikely to encode a } \\
\text { functional protein, based on available experimental } \\
\text { and comparative sequence data; overlaps with verified } \\
\text { gene BUD7/YOR299W; mutation affects bipolar } \\
\text { budding and bud site selection, though phenotype } \\
\text { could be due to the mutation's effects on BUD7 }\end{array}$ \\
\hline 50.1 & 0.033 & YPR042C & PUF2 & $\begin{array}{l}\text { mRNA-binding protein; member of the PUF protein } \\
\text { family, which is defined by the presence of Pumilio } \\
\text { homology domains that confer RNA binding activity; } \\
\text { preferentially binds mRNAs encoding membrane- } \\
\text { associated proteins; PUF2 has a paralog, JSN1, that } \\
\text { arose from the whole genome duplication }\end{array}$ \\
\hline 54.6 & 0.034 & YOR100C & CRC1 & $\begin{array}{l}\text { Mitochondrial inner membrane carnitine transporter; } \\
\text { required for carnitine-dependent transport of acetyl- } \\
\text { CoA from peroxisomes to mitochondria during fatty } \\
\text { acid beta-oxidation }\end{array}$ \\
\hline 69.1 & 0.037 & $\begin{array}{l}\text { YMR105W- } \\
\text { A }\end{array}$ & YMR105W-A & Putative protein of unknown function \\
\hline 48.4 & 0.046 & YKL053W & YKL053W & $\begin{array}{l}\text { Dubious open reading frame; unlikely to encode a } \\
\text { functional protein, based on available experimental } \\
\text { and comparative sequence data; partially overlaps } \\
\text { verified ORF ASK1 }\end{array}$ \\
\hline 46.8 & 0.049 & YBR071W & YBR071W & $\begin{array}{l}\text { Protein of unknown function found in the cytoplasm } \\
\text { and bud neck; mRNA expression may be regulated by } \\
\text { the cell cycle and/or cell wall stress; overexpression } \\
\text { of YBR071W affects endocytic protein trafficking }\end{array}$ \\
\hline
\end{tabular}




\section{References}

1. Sakula, A. Robert Koch: centenary of the discovery of the tubercle bacillus, 1882. Thorax, 1982. 37(4). 246-251.

2. Zink, A., Haas, C.J., Reischl, U. et al. Molecular analysis of skeletal tuberculosis in an ancient Egyptian population. Journal of Medical Microbiology, 2001. 50(4). 355-366.

3. Hershkovitz, I., Donoghue, H.D., Minnikin, D.E. et al. Detection and molecular characterization of 9,000-year-old Mycobacterium tuberculosis from a Neolithic settlement in the Eastern Mediterranean. PLoS One, 2008. 3(10). 3426.

4. World Health Organization. Tuberculosis. [cited 2013 27/11/2013]; Available from: http://www.who.int/topics/tuberculosis/en/.

5. Tiemersma, E.W., Van der Werf, M.J., Borgdorff, M.W. et al. Natural history of tuberculosis: duration and fatality of untreated pulmonary tuberculosis in HIV negative patients: a systematic review. PLoS One, 2011. 6(4). e17601.

6. Baydur, A. The spectrum of extrapulmonary tuberculosis. Western Journal of Medicine, 1977. 126(4). 253-262.

7. Ray, S., Talukdar, A., Kundu, S. et al. Diagnosis and management of miliary tuberculosis: current state and future perspectives. Therapeutics and Clinical Risk Management, 2013. 9. 9-26.

8. World Health Organization. Global tuberculosis report 2013. 2013.

9. World Health Organization. World Health Organization: Tuberculosis Fact sheet $N^{\circ}$ 104. 2013 February 2013 [cited 2013 18/04/2013]; Available from: http://www.who.int/mediacentre/factsheets/fs104/en/index.html.

10. Lim, E., Heffernan, H. Tuberculosis in New Zealand: Annual Report 2012. 2013; Available from:

https://surv.esr.cri.nz/PDF_surveillance/AnnTBReports/TBAnnualReport2012.p df.

11. Harries, A.D. Robert Koch and the discovery of the tubercle bacillus: the challenge of HIV and tuberculosis 125 years later. The International Journal of Tuberculosis and Lung Disease, 2008. 12(3). 241-249.

12. Ellis, R.C. and Zabrowarny, L.A. Safer staining method for acid fast bacilli. Journal of Clinical Pathology, 1993. 46(6). 559-560.

13. Stahl, D.A. and Urbance, J.W. The division between fast-and slow-growing species corresponds to natural relationships among the mycobacteria. Journal of Bacteriology, 1990. 172(1). 116-124.

14. Shinnick, T.M. and Good, R.C. Mycobacterial taxonomy. European Journal of Clinical Microbiology and Infectious Diseases, 1994. 13(11). 884-901.

15. Kothavade, R.J., Dhurat, R.S., Mishra, S.N. et al. Clinical and laboratory aspects of the diagnosis and management of cutaneous and subcutaneous infections caused by rapidly growing mycobacteria. European Journal of Clinical Microbiology \& Infectious Diseases, 2013. 32(2). 161-188.

16. Abdallah, A.M., Rashid, M., Adroub, S.A. et al. Complete Genome Sequence of Mycobacterium phlei Type Strain RIVM601174. Journal of Bacteriology, 2012. 194(12). 3284-3285.

17. Koranyi, K.I. and Ranalli, M.A. Mycobacterium aurum bacteremia in an immunocompromised child. Pediatric Infectious Disease Journal, 2003. 22(12). 1108-1109. 
18. Tsukamura, M. Properties of Mycobacterium smegmatis Freshly Isolated from Soil. Japanese Journal of Microbiology, 1976. 20(4). 355-356.

19. Newton, J.A., Jr., Weiss, P.J., Bowler, W.A. et al. Soft-Tissue Infection Due to Mycobacterium smegmatis: Report of Two Cases. Clinical Infectious Diseases, 1993. 16(4). 531-533.

20. Husson, R., Homologous Recombination in Mycobacterium smegmatis, in Mycobacteria Protocols, T. Parish and N. Stoker, Editors. 1998, Humana Press. 199-206.

21. Chaturvedi, V., Dwivedi, N., Tripathi, R.P. et al. Evaluation of Mycobacterium smegmatis as a possible surrogate screen for selecting molecules active against multi-drug resistant Mycobacterium tuberculosis. Journal of General and Applied Microbiology, 2007. 53(6). 333-337.

22. Shiloh, M.U. and Champion, P.A. To catch a killer. What can mycobacterial models teach us about Mycobacterium tuberculosis pathogenesis? Current Opinion in Microbiology, 2010. 13(1). 86-92.

23. Zheng, H., Lu, L., Wang, B. et al. Genetic Basis of Virulence Attenuation Revealed by Comparative Genomic Analysis of Mycobacterium tuberculosis Strain H37Ra versus H37Rv. PLoS One, 2008. 3(6). e2375.

24. Philips, J.A. and Ernst, J.D. Tuberculosis pathogenesis and immunity. Annual Review of Pathology: Mechanisms of Disease, 2012. 7. 353-384.

25. Grosset, J. Mycobacterium tuberculosis in the Extracellular Compartment: an Underestimated Adversary. Antimicrobial Agents and Chemotherapy, 2003. 47(3). 833-836.

26. Russell, D.G. Mycobacterium tuberculosis: here today, and here tomorrow. Nature Reviews Molecular Cell Biology, 2001. 2(8). 569-586.

27. World health Organization. Treatment of tuberculosis: guidelines - 4th ed. 2009.

28. Moonan, P.K., Quitugua, T.N., Pogoda, J.M. et al. Does directly observed therapy (DOT) reduce drug resistant tuberculosis? BMC Public Health, 2011. 11. 19.

29. Wehrli, W., Knusel, F., Schmid, K. et al. Interaction of rifamycin with bacterial RNA polymerase. Proceedings of the National Academy of Sciences, 1968. 61(2). 667-673.

30. Zhang, Y., Wade, M.M., Scorpio, A. et al. Mode of action of pyrazinamide: disruption of Mycobacterium tuberculosis membrane transport and energetics by pyrazinoic acid. Journal of Antimicrobial Chemotherapy, 2003. 52(5). 790795.

31. Ramaswamy, S.V., Amin, A.G., Göksel, S. et al. Molecular Genetic Analysis of Nucleotide Polymorphisms Associated with Ethambutol Resistance in Human Isolates of Mycobacterium tuberculosis. Antimicrobial Agents and Chemotherapy, 2000. 44(2). 326-336.

32. Vilcheze, C., Wang, F., Arai, M. et al. Transfer of a point mutation in Mycobacterium tuberculosis inhA resolves the target of isoniazid. Nature Medicine (New York, NY, United States), 2006. 12(9). 1027-1029.

33. World Health Organization. Guidelines for the programmatic management of drug-resistant tuberculosis - 2011 update. 2011.

34. Centers for Disease Control. Treatment of Tuberculosis, American Thoracic Society, CDC, and Infectious Diseases Society of America. Morbidity and Mortality Weekly Report, 2003. 52(RR-11). 1-77. 
35. Cox, H.S., Morrow, M., and Deutschmann, P.W. Long term efficacy of DOTS regimens for tuberculosis: systematic review. British Medical Journal., 2008. 336(7642). 484-487.

36. Donald, P.R. and Van Helden, P.D. The global burden of tuberculosiscombating drug resistance in difficult times. New England Journal of Medicine, 2009. 360(23). 2393-2395.

37. World Health Organization. Global tuberculosis report 2012. 2012.

38. Wood, A.J.J., Piscitelli, S.C., and Gallicano, K.D. Interactions among drugs for HIV and opportunistic infections. New England Journal of Medicine, 2001. 344(13). 984-996.

39. La Porte, C.J., Colbers, E.P., Bertz, R. et al. Pharmacokinetics of adjusted-dose lopinavir-ritonavir combined with rifampin in healthy volunteers. Antimicrobial Agents and Chemotherapy, 2004. 48(5). 1553-1560.

40. Zumla, A., Nahid, P., and Cole, S.T. Advances in the development of new tuberculosis drugs and treatment regimens. Nature Reviews Drug Discovery, 2013. 12(5). 388-404.

41. Mahairas, G.G., Sabo, P.J., Hickey, M.J. et al. Molecular analysis of genetic differences between Mycobacterium bovis BCG and virulent $M$. bovis. Journal of Bacteriology, 1996. 178(5). 1274-1282.

42. Garnier, T., Eiglmeier, K., Camus, J.C. et al. The complete genome sequence of Mycobacterium bovis. Proceedings of the National Academy of Sciences, 2003. 100(13). 7877-7882.

43. Colditz, G.A., Brewer, T.F., Berkey, C.S. et al. Efficacy of BCG vaccine in the prevention of tuberculosis. Meta-analysis of the published literature. The Journal of the America Medical Association, 1994. 271(9). 698-702.

44. Zetterström, R. Selman A. Waksman (1888-1973) Nobel Prize in 1952 for the discovery of streptomycin, the first antibiotic effective against tuberculosis. Acta Paediatrica, 2007. 96(2). 317-319.

45. Giedraitiene, A., Vitkauskiene, A., Naginiene, R. et al. Antibiotic resistance mechanisms of clinically important bacteria. Medicina (Kaunas), 2011. 47(3). 137-146.

46. Almeida Da Silva, P.E. and Palomino, J.C. Molecular basis and mechanisms of drug resistance in Mycobacterium tuberculosis: classical and new drugs. Journal of Antimicrobial Chemotherapy, 2011. 66(7). 1417-1430.

47. Hazbón, M.H., Brimacombe, M., Bobadilla del Valle, M. et al. Population Genetics Study of Isoniazid Resistance Mutations and Evolution of MultidrugResistant Mycobacterium tuberculosis. Antimicrobial Agents and Chemotherapy, 2006. 50(8). 2640-2649.

48. Zhang, Y., Heym, B., Allen, B. et al. The catalase-peroxidase gene and isoniazid resistance of Mycobacterium tuberculosis. Nature, 1992. 358(6387). 591-593.

49. Telenti, A., Imboden, P., Marchesi, F. et al. Detection of rifampicin-resistance mutations in Mycobacterium tuberculosis. The Lancet, 1993. 341(8846). 647650.

50. Scorpio, A., Lindholm-Levy, P., Heifets, L. et al. Characterization of pncA mutations in pyrazinamide-resistant Mycobacterium tuberculosis. Antimicrobial Agents and Chemotherapy, 1997. 41(3). 540-543.

51. Safi, H., Sayers, B., Hazbón, M.H. et al. Transfer of embB Codon 306 Mutations into Clinical Mycobacterium tuberculosis Strains Alters Susceptibility to 
Ethambutol, Isoniazid, and Rifampin. Antimicrobial Agents and Chemotherapy, 2008. 52(6). 2027-2034.

52. World Health Organization. Definitions and reporting framework for tuberculosis -2013 revision. 2013.

53. Cole, S.T. and Alzari, P.M. Towards new tuberculosis drugs. Biochemical Society Transactions, 2007. 35(Pt 5). 1321-1324.

54. Shafer, R.W., Small, P.M., Larkin, C. et al. Temporal trends and transmission patterns during the emergence of multidrug-resistant tuberculosis in New York City: a molecular epidemiologic assessment. Journal of Infectious Diseases, 1995. 171(1). 170-176.

55. Velayati, A.A., Masjedi, M.R., Farnia, P. et al. Emergence of new forms of totally drug-resistant tuberculosis bacilli: super extensively drug-resistant tuberculosis or totally drug-resistant strains in Iran. Chest, 2009. 136(2). 420425.

56. Udwadia, Z.F., Amale, R.A., Ajbani, K.K. et al. Totally Drug-Resistant Tuberculosis in India. Clinical Infectious Diseases, 2011.

57. Migliori, G.B., De Iaco, G., Besozzi, G. et al. First tuberculosis cases in Italy resistant to all tested drugs. Euro Surveillance, 2007. 12(5). E070517 070511.

58. World Health Organization. Drug-resistant tuberculosis. 2012 [cited 2013 3/09/2013]; Available from: http://www.who.int/tb/challenges/mdr/tdrfaqs/en/.

59. Centers for Disease Control and Prevention. Emergence of Mycobacterium tuberculosis with extensive resistance to second-line drugs--worldwide, 20002004. MMWR Morbidity Mortality Weekly Report, 2006. 55(11). 301-305.

60. Torun, T., Gungor, G., Ozmen, I. et al. Side effects associated with the treatment of multidrug-resistant tuberculosis. The International Journal of Tuberculosis and Lung Disease, 2005. 9(12). 1373-1377.

61. Gandhi, N.R., Moll, A., Sturm, A.W. et al. Extensively drug-resistant tuberculosis as a cause of death in patients co-infected with tuberculosis and HIV in a rural area of South Africa. Lancet, 2006. 368(9547). 1575-1580.

62. Bissielo, A., Lim, E., and Heffernan, H.,. Tuberculosis in New Zealand: Annual Report 2010,. 2011; Available from: https://surv.esr.cri.nz/PDF_surveillance/AnnTBReports/TBAnnualReport2010.p df.

63. Singh, R., Manjunatha, U., Boshoff, H.I.M. et al. PA-824 Kills Nonreplicating Mycobacterium tuberculosis by Intracellular NO Release. Science, 2008. 322(5906). 1392-1395.

64. Tahlan, K., Wilson, R., Kastrinsky, D.B. et al. SQ109 targets MmpL3, a membrane transporter of trehalose monomycolate involved in mycolic acid donation to the cell wall core of Mycobacterium tuberculosis. Antimicrobial Agents and Chemotherapy, 2012. 56(4). 1797-1809.

65. Leach, K.L., Brickner, S.J., Noe, M.C. et al. Linezolid, the first oxazolidinone antibacterial agent. Annals of the New York Academy of Sciences, 2011. 1222. 49-54.

66. Koul, A., Dendouga, N., Vergauwen, K. et al. Diarylquinolines target subunit c of mycobacterial ATP synthase. Nature Chemical Biology, 2007. 3(6). 323-324.

67. Cohen, J. Approval of Novel TB Drug Celebrated-With Restraint. Science, 2013. 339(6116). 130.

68. Lienhardt, C., Vernon, A., and Raviglione, M.C. New drugs and new regimens for the treatment of tuberculosis: review of the drug development pipeline and 
implications for national programmes. Current Opinion in Pulmonary Medicine, 2010. 16(3). 186-193.

69. Imhoff, J.F., Labes, A., and Wiese, J. Bio-mining the microbial treasures of the ocean: New natural products. Biotechnology Advances, 2011. 29(5). 468-482.

70. Kusari, S., Hertweck, C., and Spiteller, M. Chemical ecology of endophytic fungi: origins of secondary metabolites. Chemistry \& Biology, 2012. 19(7). 792-798.

71. Baltz, R.H. Renaissance in antibacterial discovery from actinomycetes. Current Opinion in Pharmacology, 2008. 8(5). 557-563.

72. Sudek, S., Lopanik, N.B., Waggoner, L.E. et al. Identification of the Putative Bryostatin Polyketide Synthase Gene Cluster from "Candidatus Endobugula sertula”, the Uncultivated Microbial Symbiont of the Marine Bryozoan Bugula neritina. Journal of Natural Products, 2006. 70(1). 67-74.

73. Stierle, A., Strobel, G., and Stierle, D. Taxol and taxane production by Taxomyces andreanae, an endophytic fungus of Pacific yew. Science, 1993. 260(5105). 214-216.

74. Walsh, C.T. Combinatorial biosynthesis of antibiotics: challenges and opportunities. Chembiochem, 2002. 3(2-3). 125-134.

75. Cane, D.E., Walsh, C.T., and Khosla, C. Harnessing the biosynthetic code: combinations, permutations, and mutations. Science, 1998. 282(5386). 63-68.

76. Newman, D.J., Cragg, G.M., and Snader, K.M. The influence of natural products upon drug discovery. Natural Product Reports, 2000. 17(3). 215-234.

77. Newman, D.J. and Cragg, G.M. Natural Products As Sources of New Drugs over the 30 Years from 1981 to 2010. Journal of Natural Products, 2012. 75(3). 311-335.

78. Waksman, S.A., Reilly, H.C., and Johnstone, D.B. Isolation of Streptomycinproducing Strains of Streptomyces griseus. Journal of Bacteriology, 1946. 52(3). 393-397.

79. Zatman, L.J., Kaplan, N.O., Colowick, S.P. et al. Effect of isonicotinic acid hydrazide on diphosphopyridine nucleotidases. Journal of Biological Chemistry, 1954. 209(2). 453-466.

80. Lester, W. Rifampin: a semisynthetic derivative of rifamycin-a prototype for the future. Annual Review of Microbiology, 1972. 26. 85-102.

81. Bala, S., Khanna, R., Dadhwal, M. et al. Reclassification of Amycolatopsis mediterranei DSM 46095 as Amycolatopsis rifamycinica sp. nov. International Journal of Systemic and Evolutionary Microbiolgy, 2004. 54(Pt 4). 1145-1149.

82. O'Connor, J.A. Para-aminosalicylic acid in the Treatment of Tuberculosis. Postgrad Medical Journal, 1948. 24(275). 455-462.

83. Di Perri, G. and Bonora, S. Which agents should we use for the treatment of multidrug-resistant Mycobacterium tuberculosis? Journal of Antimicrobial Chemotherapy, 2004. 54(3). 593-602.

84. Murdoch, J.M., Sleigh, J.D., and Frazer, S.C. Cycloserine in treatment of infection of urinary tract. British Medical Journal, 1959. 2(5159). 1055-1058.

85. Abou-Zeid, A.A., Salem, H.M., and Essa, A.I. Factors influencing the production of kanamycin by Streptomyces canus. Zeitschrift fur allgemeine Mikrobiologie, 1971. 11(6). 469-473.

86. Dirlam, J.P., Belton, A.M., Birsner, N.C. et al. Cyclic homopentapeptides 1. Analogs of tuberactinomycins and capreomycin with activity against vancomycin-resistant enterococci and Pasteurella. Bioorganic \& Medicinal Chemistry Letters, 1997. 7(9). 1139-1144. 
87. Lester, W. Rifampin: a semisynthetic derivative of rifamycin--a prototype for the future. Annual Review of Microbiology, 1972. 26. 85-102.

88. Joklik, W.K. The story of penicillin: the view from Oxford in the early 1950s. The FASEB Journal, 1996. 10(4). 525-528.

89. Blunt, J.W., Copp, B.R., Keyzers, R.A. et al. Marine natural products. Natural Product Reports, 2013. 30(2). 237-323.

90. Emelyanov, A., Fedoseev, G., Krasnoschekova, O. et al. Treatment of asthma with lipid extract of New Zealand green-lipped mussel: a randomised clinical trial. European Respitoray Journal, 2002. 20(3). 596-600.

91. Brien, S., Prescott, P., Coghlan, B. et al. Systematic review of the nutritional supplement Perna Canaliculus (green-lipped mussel) in the treatment of osteoarthritis. Quarterly Journal of Medicine, 2008. 101(3). 167-179.

92. Hood, K.A., West, L.M., Rouwe, B. et al. Peloruside A, a novel antimitotic agent with paclitaxel-like microtubule- stabilizing activity. Cancer Research, 2002. 62(12). 3356-3360.

93. Molinski, T.F., Dalisay, D.S., Lievens, S.L. et al. Drug development from marine natural products. Nature Reviews Drug Discovery, 2009. 8(1). 69-85.

94. Jain, S. and Vahdat, L.T. Eribulin Mesylate. Clinical Cancer Research, 2011. 17(21). 6615-6622.

95. Bai, R.L., Paull, K.D., Herald, C.L. et al. Halichondrin B and homohalichondrin $B$, marine natural products binding in the vinca domain of tubulin. Discovery of tubulin-based mechanism of action by analysis of differential cytotoxicity data. Journal of Biological Chemistry, 1991. 266(24). 15882-15889.

96. Mincer, T.J., Jensen, P.R., Kauffman, C.A. et al. Widespread and Persistent Populations of a Major New Marine Actinomycete Taxon in Ocean Sediments. Applied and Environmental Microbiology, 2002. 68(10). 5005-5011.

97. Feling, R.H., Buchanan, G.O., Mincer, T.J. et al. Salinosporamide A: A Highly Cytotoxic Proteasome Inhibitor from a Novel Microbial Source, a Marine Bacterium of the New Genus Salinospora. Angewandte Chemie International Edition, 2003. 42(3). 355-357.

98. Mayer, A.M.S., Glaser, K.B., Cuevas, C. et al. The odyssey of marine pharmaceuticals: a current pipeline perspective. Trends in pharmacological sciences, 2010. 31(6). 255-265.

99. Webster, N.S. and Taylor, M.W. Marine sponges and their microbial symbionts: love and other relationships. Environmental Microbiology, 2012. 14(2). 335346.

100. Rodríguez, I.I. and Rodríguez, A.D. Homopseudopteroxazole, a New Antimycobacterial Diterpene Alkaloid from Pseudopterogorgia elisabethae. Journal of Natural Products, 2003. 66(6). 855-857.

101. Arai, M., Sobou, M., Vilcheze, C. et al. Halicyclamine A, a marine spongean alkaloid as a lead for anti-tuberculosis agent. Bioorganic \& Medicinal Chemistry Letters, 2008. 16(14). 6732-6736.

102. Wei, X., Rodríguez, A.D., Wang, Y. et al. Novel ring B abeo-sterols as growth inhibitors of Mycobacterium tuberculosis isolated from a Caribbean Sea sponge, Svenzea zeai. Tetrahedron Letters, 2007. 48(50). 8851-8854.

103. Kong, F., Singh, M.P., and Carter, G.T. Pseudopyronines A and B, $\alpha$-Pyrones Produced by a Marine Pseudomonas sp. F92S91, and Evidence for the Conversion of 4-Hydroxy- $\alpha$-pyrone to 3-Furanone. Journal of Natural Products, 2005. 68(6). 920-923. 
104. Giddens, A.C., Nielsen, L., Boshoff, H.I. et al. Natural product inhibitors of fatty acid biosynthesis: synthesis of the marine microbial metabolites pseudopyronines $A$ and $B$ and evaluation of their anti-infective activities. Tetrahedron, 2008. 64(7). 1242-1249.

105. Sakai, R., Higa, T., Jefford, C.W. et al. Manzamine A, a novel antitumor alkaloid from a sponge. Journal of the American Chemical Society, 1986. 108(20). 6404-6405.

106. Ichiba, T., Sakai, R., Kohmoto, S. et al. New manzamine alkaloids from a sponge of the genus Xestospongia. Tetrahedron Letters, 1988. 29(25). 30833086.

107. Rao, K.V., Santarsiero, B.D., Mesecar, A.D. et al. New manzamine alkaloids with activity against infectious and tropical parasitic diseases from an Indonesian sponge. Journal of Natural Products, 2003. 66(6). 823-828.

108. Song, F., Liu, X., Guo, H. et al. Brevianamides with antitubercular potential from a marine-derived isolate of Aspergillus versicolor. Organic Letters, 2012. 14(18). 4770-4773.

109. Pruksakorn, P., Arai, M., Kotoku, N. et al. Trichoderins, novel aminolipopeptides from a marine sponge-derived Trichoderma sp., are active against dormant mycobacteria. Bioorganic \& Medicinal Chemistry Letters, 2010. 20(12). 3658-3663.

110. Keller, S., Nicholson, G., Drahl, C. et al. Abyssomicins $G$ and $H$ and atropAbyssomicin C from the Marine Verrucosispora Strain AB-18-032[dagger]. Journal of Antibiotics, 2007. 60(6). 391-394.

111. Freundlich, J.S., Lalgondar, M., Wei, J.-R. et al. The Abyssomicin C family as in vitro inhibitors of Mycobacterium tuberculosis. Tuberculosis, 2010. 90(5). 298300.

112. Potterat, O. and Hamburger, M. Concepts and technologies for tracking bioactive compounds in natural product extracts: generation of libraries, and hyphenation of analytical processes with bioassays. Natural Product Reports, 2013. 30(4). 546-564.

113. Showalter, H.D. and Denny, W.A. A roadmap for drug discovery and its translation to small molecule agents in clinical development for tuberculosis treatment. Tuberculosis (Edinb), 2008. 88 Suppl 1. 3-17.

114. Li, J.W.H. and Vederas, J.C. Drug Discovery and Natural Products: End of an Era or an Endless Frontier? Science, 2009. 325(5937). 161-165.

115. Payne, D.J., Gwynn, M.N., Holmes, D.J. et al. Drugs for bad bugs: confronting the challenges of antibacterial discovery. Nature Reviews Drug Discovery, 2007. 6(1). 29-40.

116. Kallifatidis, G., Hoepfner, D., Jaeg, T. et al. The marine natural product manzamine A targets vacuolar ATPases and inhibits autophagy in pancreatic cancer cells. Marine Drugs, 2013. 11(9). 3500-3516.

117. Argyrou, A., Jin, L., Siconilfi-Baez, L. et al. Proteome-wide profiling of isoniazid targets in Mycobacterium tuberculosis. Biochemistry, 2006. 45(47). 13947-13953.

118. Andries, K., Verhasselt, P., Guillemont, J. et al. A diarylquinoline drug active on the ATP synthase of Mycobacterium tuberculosis. Science, 2005. 307(5707). 223-227.

119. Lum, P.Y., Armour, C.D., Stepaniants, S.B. et al. Discovering modes of action for therapeutic compounds using a genome-wide screen of yeast heterozygotes. Cell, 2004. 116(1). 121-137. 
120. Saccharomyces Genome Deletion Project. 2007 1/12/2013]; Available from: http://www-sequence.stanford.edu/group/yeast_deletion_project/deletions3.html.

121. Shoemaker, D.D., Lashkari, D.A., Morris, D. et al. Quantitative phenotypic analysis of yeast deletion mutants using a highly parallel molecular bar-coding strategy. Nature Genetics, 1996. 14(4). 450-456.

122. Luesch, H. Towards high-throughput characterization of small molecule mechanisms of action. Molecular BioSystems, 2006. 2(12). 609-620.

123. Giaever, G., Shoemaker, D.D., Jones, T.W. et al. Genomic profiling of drug sensitivities via induced haploinsufficiency. Nature Genetics, 1999. 21(3). 278283.

124. Giaever, G., Flaherty, P., Kumm, J. et al. Chemogenomic profiling: identifying the functional interactions of small molecules in yeast. Proceedings of the National Academy of Sciences, 2004. 101(3). 793-798.

125. Hughes, T.R., Marton, M.J., Jones, A.R. et al. Functional discovery via a compendium of expression profiles. Cell, 2000. 102(1). 109-126.

126. Ooi, S.L., Shoemaker, D.D., and Boeke, J.D. DNA helicase gene interaction network defined using synthetic lethality analyzed by microarray. Nature Genetics, 2003. 35(3). 277-286.

127. Tong, A.H., Evangelista, M., Parsons, A.B. et al. Systematic genetic analysis with ordered arrays of yeast deletion mutants. Science, 2001. 294(5550). 23642368.

128. Srivastava, V., Rouanet, C., Srivastava, R. et al. Macrophage-specific Mycobacterium tuberculosis genes: identification by green fluorescent protein and kanamycin resistance selection. Microbiology, 2007. 153(Pt 3). 659-666.

129. Guilhot, C., Otal, I., Van Rompaey, I. et al. Efficient transposition in mycobacteria: construction of Mycobacterium smegmatis insertional mutant libraries. Journal of Bacteriology, 1994. 176(2). 535-539.

130. Taneja, N.K. and Tyagi, J.S. Resazurin reduction assays for screening of antitubercular compounds against dormant and actively growing Mycobacterium tuberculosis, Mycobacterium bovis BCG and Mycobacterium smegmatis. Journal of Antimicrobial Chemotherapy, 2007. 60(2). 288-293.

131. Bassett, I.M., Lun, S., Bishai, W.R. et al. Detection of inhibitors of phenotypically drug-tolerant Mycobacterium tuberculosis using an in vitro bactericidal screen. Journal of Microbiology, 2013. 51(5). 651-658.

132. West, L. The Isolation of Secondary Metabolites from New Zealand Marine Sponges, Ph.D. thesis. 2001, Victoria University of Wellington.

133. Centers for Disease Control. Antibiotic resistance threats in the United States, 2013. 20132013 [cited 2013 06/12/2013]; Available from: http://www.cdc.gov/drugresistance/threat-report-2013/pdf/ar-threats-2013508.pdf.

134. Heffernan, H. and Bakker, S. Annual survey of methicillin-resistant Staphylococcus aureus (MRSA). 2013, Institute of Environmental Science \& Research Limited.

135. Heffernan, H. and Woodhouse, R. Annual survey of extended-spectrum $\beta$ lactamase (ESBL)-producing Enterobacteriaceae. 2013, Institute of Environmental Science \& Research Limited.

136. Lallier, L.E., McMeel, O., Greiber, T. et al. Access to and use of marine genetic resources: understanding the legal framework. Natural Product Reports, 2014. 31(5). 612-616. 
137. Brower, V. Back to Nature: Extinction of Medicinal Plants Threatens Drug Discovery. Journal of the National Cancer Institute, 2008. 100(12). 838-839.

138. Page, M., West, L., Northcote, P. et al. Spatial and temporal variability of cytotoxic metabolites in populations of the New Zealand sponge Mycale hentscheli. Journal of Chemical Ecology, 2005. 31(5). 1161-1174.

139. Baltz, R.H. Antimicrobials from actinomycetes: Back to the future. Microbe, 2007. 2(3). 125-131.

140. DiMasi, J.A., Hansen, R.W., and Grabowski, H.G. The price of innovation: new estimates of drug development costs. Journal of Health Economics, 2003. 22(2). 151-185.

141. Kola, I. and Landis, J. Can the pharmaceutical industry reduce attrition rates? Nature Reviews Drug Discovery, 2004. 3(8). 711-716.

142. Braine, T. Race against time to develop new antibiotics. Bulletin of the World Health Organization, 2011(89). 88-89.

143. Coates, A.R. and Hu, Y. Novel approaches to developing new antibiotics for bacterial infections. British Journal of Pharmacology, 2007. 152(8). 1147-1154.

144. Malik, N.N. Drug discovery: past, present and future. Drug Discovery Today, 2008. 13(21-22). 909-912.

145. Chong, C.R. and Sullivan, D.J. New uses for old drugs. Nature, 2007. 448(7154). 645-646.

146. Wijkmans, J.C. and Beckett, R.P. Combinatorial chemistry in anti-infectives research. Drug Discovery Today, 2002. 7(2). 126-132.

147. Tajabadi, F., Campitelli, M., and Quinn, R. Scaffold Flatness: Reversing the Trend. Springer Science Reviews, 2013. 1(1-2). 141-151.

148. World Health Organization. Antimicrobial resistance, Fact sheet $N^{\circ} 194.2013$ 2013 [cited 2013 2/12/2013]; Available from:

http://www.who.int/mediacentre/factsheets/fs194/en/.

149. Butler, M.S., Blaskovich, M.A., and Cooper, M.A. Antibiotics in the clinical pipeline in 2013. Journal of Antibiotics, 2013. 66(10). 571-591.

150. Wang, J., Soisson, S.M., Young, K. et al. Platensimycin is a selective FabF inhibitor with potent antibiotic properties. Nature, 2006. 441(7091). 358-361.

151. Wang, J., Kodali, S., Lee, S.H. et al. Discovery of platencin, a dual FabF and FabH inhibitor with in vivo antibiotic properties. Proceedings of the National Academy of Sciences, 2007. 104(18). 7612-7616.

152. Bugni, T.S., Richards, B., Bhoite, L. et al. Marine Natural Product Libraries for High-Throughput Screening and Rapid Drug Discovery. Journal of Natural Products, 2008. 71(6). 1095-1098.

153. Eustaquio, A.S., Nam, S.J., Penn, K. et al. The discovery of salinosporamide K from the marine bacterium "Salinispora pacifica" by genome mining gives insight into pathway evolution. Chembiochem, 2011. 12(1). 61-64.

154. Laureti, L., Song, L., Huang, S. et al. Identification of a bioactive 51-membered macrolide complex by activation of a silent polyketide synthase in Streptomyces ambofaciens. Proceedings of the National Academy of Sciences, 2011. 108(15). 6258-6263.

155. Miller, C.H., Nisa, S., Dempsey, S. et al. Modifying Culture Conditions in Chemical Library Screening Identifies Alternative Inhibitors of Mycobacteria. Antimicrobial Agents and Chemotherapy, 2009. 53(12). 5279-5283.

156. Stover, C.K., Warrener, P., VanDevanter, D.R. et al. A small-molecule nitroimidazopyran drug candidate for the treatment of tuberculosis. Nature, 2000. 405(6789). 962-966. 
157. Andreu, N., Fletcher, T., Krishnan, N. et al. Rapid measurement of antituberculosis drug activity in vitro and in macrophages using bioluminescence. Journal of Antimicrobial Chemotherapy, 2012. 67(2). 404-414.

158. Chen, H., Kovar, J., Sissons, S. et al. A cell-based immunocytochemical assay for monitoring kinase signaling pathways and drug efficacy. Analytical Biochemistry, 2005. 338(1). 136-142.

159. White, E.L., Southworth, K., Ross, L. et al. A Novel Inhibitor of Mycobacterium tuberculosis Pantothenate Synthetase. Journal of Biomolecular Screening, 2007. 12(1). 100-105.

160. Muramatsu, Y., Ishii, M.M., and Inukai, M. Studies on novel bacterial translocase I inhibitors, A-500359s. II. Biological activities of A-500359 A, C, D and G. Journal of Antibiotics, 2003. 56(3). 253-258.

161. Palomino, J.C., Martin, A., Camacho, M. et al. Resazurin microtiter assay plate: simple and inexpensive method for detection of drug resistance in Mycobacterium tuberculosis. Antimicrobial Agents and Chemotherapy, 2002. 46(8). 2720-2722.

162. Juliane, O., Mai Ann, B., Garrett, C.M. et al. A Dual Read-Out Assay to Evaluate the Potency of Compounds Active against Mycobacterium tuberculosis. PLoS One, 2013. 8(4).

163. Koul, A., Arnoult, E., Lounis, N. et al. The challenge of new drug discovery for tuberculosis. Nature, 2011. 469(7331). 483-490.

164. Zhang, J.H., Chung, T.D.Y., and Oldenburg, K.R. A Simple Statistical Parameter for Use in Evaluation and Validation of High Throughput Screening Assays. Journal of Biomolecular Screening, 1999. 4(2). 67-73.

165. Desbois, A.P. and Smith, V.J. Antibacterial free fatty acids: activities, mechanisms of action and biotechnological potential. Applied Microbiology and Biotechnology, 2010. 85(6). 1629-1642.

166. Thormar, H. and Hilmarsson, H. The role of microbicidal lipids in host defense against pathogens and their potential as therapeutic agents. Chemistry and Physics of Lipids, 2007. 150(1). 1-11.

167. Takigawa, H., Nakagawa, H., Kuzukawa, M. et al. Deficient production of hexadecenoic acid in the skin is associated in part with the vulnerability of atopic dermatitis patients to colonization by Staphylococcus aureus. Dermatology, 2005. 211(3). 240-248.

168. Fluhr, J.W., Kao, J., Jain, M. et al. Generation of free fatty acids from phospholipids regulates stratum corneum acidification and integrity. Journal of Investigative Dermatology, 2001. 117(1). 44-51.

169. Desbois, A.P., Lebl, T., Yan, L. et al. Isolation and structural characterisation of two antibacterial free fatty acids from the marine diatom, Phaeodactylum tricornutum. Applied Microbiology and Biotechnology, 2008. 81(4). 755-764.

170. Sandoval-Montemayor, N.E., Garcia, A., Elizondo-Trevino, E. et al. Chemical composition of hexane extract of Citrus aurantifolia and anti-Mycobacterium tuberculosis activity of some of its constituents. Molecules, 2012. 17(9). 1117311184.

171. Seidel, V. and Taylor, P.W. In vitro activity of extracts and constituents of Pelagonium against rapidly growing mycobacteria. International Journal of Antimicrobial Agents, 2004. 23(6). 613-619.

172. Yff, B.T., Lindsey, K.L., Taylor, M.B. et al. The pharmacological screening of Pentanisia prunelloides and the isolation of the antibacterial compound palmitic acid. Journal of Ethnopharmacology, 2002. 79(1). 101-107. 
173. Kabara, J.J., Swieczkowski, D.M., Conley, A.J. et al. Fatty acids and derivatives as antimicrobial agents. Antimicrobial Agents and Chemotherapy, 1972. 2(1). 23-28.

174. Feldlaufer, M.F., Knox, D.A., Lusby, W.R. et al. Antimicrobial activity of fatty acids against Bacillus larvae, the causative agent of American foulbrood disease. Apidologie, 1993. 24(2). 95-99.

175. Kabara, J.J. and Vrable, R. Antimicrobial lipids: natural and synthetic fatty acids and monoglycerides. Lipids, 1977. 12(9). 753-759.

176. Zheng, C.J., Yoo, J.S., Lee, T.-G. et al. Fatty acid synthesis is a target for antibacterial activity of unsaturated fatty acids. FEBS Letters, 2005. 579(23). 5157-5162.

177. Ouattara, B., Simard, R.E., Holley, R.A. et al. Antibacterial activity of selected fatty acids and essential oils against six meat spoilage organisms. International Journal Food Microbiology, 1997. 37(2-3). 155-162.

178. Fischer, C.L., Drake, D.R., Dawson, D.V. et al. Antibacterial activity of sphingoid bases and fatty acids against Gram-positive and Gram-negative bacteria. Antimicrobial Agents and Chemotherapy, 2012. 56(3). 1157-1161.

179. Shin, S.Y., Bajpai, V.K., Kim, H.R. et al. Antibacterial activity of eicosapentaenoic acid (EPA) against foodborne and food spoilage microorganisms. LWT - Food Science and Technology, 2007. 40(9). 1515-1519.

180. Greenway, D.L. and Dyke, K.G. Mechanism of the inhibitory action of linoleic acid on the growth of Staphylococcus aureus. Journal of General Microbiology, 1979. 115(1). 233-245.

181. Chamberlain, N.R., Mehrtens, B.G., Xiong, Z. et al. Correlation of carotenoid production, decreased membrane fluidity, and resistance to oleic acid killing in Staphylococcus aureus 18Z. Infection and Immunity, 1991. 59(12). 4332-4337.

182. Sheu, C.W. and Freese, E. Effects of fatty acids on growth and envelope proteins of Bacillus subtilis. Journal of Bacteriology, 1972. 111(2). 516-524.

183. Kenny, J.G., Ward, D., Josefsson, E. et al. The Staphylococcus aureus Response to Unsaturated Long Chain Free Fatty Acids: Survival Mechanisms and Virulence Implications. PLoS One, 2009. 4(2). e4344.

184. Rottenberg, H. and Hashimoto, K. Fatty acid uncoupling of oxidative phosphorylation in rat liver mitochondria. Biochemistry, 1986. 25(7). 17471755.

185. Boyaval, P., Corre, C., Dupuis, C. et al. Effects of free fatty acids on propionic acid bacteria. Lait, 1995. 75(1). 17-29.

186. Bruce, A., Alexander, J., Julian, L. et al. Molecular Biology of the Cell. 4th edition. Electron-Transport Chains and Their Proton Pumps. 2002, New York: Garland Science.

187. Wieckowski, M.R. and Wojtczak, L. Fatty acid-induced uncoupling of oxidative phosphorylation is partly due to opening of the mitochondrial permeability transition pore. FEBS Letters, 1998. 423(3). 339-342.

188. Beck, V., Jaburek, M., Demina, T. et al. Polyunsaturated fatty acids activate human uncoupling proteins 1 and 2 in planar lipid bilayers. The FASEB Journal, 2007. 21(4). 1137-1144.

189. Won, S.R., Hong, M.J., Kim, Y.M. et al. Oleic acid: an efficient inhibitor of glucosyltransferase. FEBS Letters, 2007. 581(25). 4999-5002.

190. Hamel, F.G. Preliminary report: inhibition of cellular proteasome activity by free fatty acids. Metabolism, 2009. 58(8). 1047-1049. 
191. Zheng, C.J., Yoo, J.S., Lee, T.G. et al. Fatty acid synthesis is a target for antibacterial activity of unsaturated fatty acids. FEBS Letters, 2005. 579(23). 5157-5162.

192. Sado-Kamdem, S.L., Vannini, L., and Guerzoni, M.E. Effect of $\alpha$-linolenic, capric and lauric acid on the fatty acid biosynthesis in Staphylococcus aureus. International Journal of Food Microbiology, 2009. 129(3). 288-294.

193. Clarke, S.R., Mohamed, R., Bian, L. et al. The Staphylococcus aureus Surface Protein IsdA Mediates Resistance to Innate Defenses of Human Skin. Cell Host \& Microbe, 2007. 1(3). 199-212.

194. Quevrain, E., Barnathan, G., Meziane, T. et al. New 2-methyl-13-icosenoic acid from the temperate calcisponge Leuconia johnstoni. Lipids, 2012. 47(4). 345353.

195. Wayne, L.G. and Hayes, L.G. An in vitro model for sequential study of shiftdown of Mycobacterium tuberculosis through two stages of nonreplicating persistence. Infection and Immunity, 1996. 64(6). 2062-2069.

196. Karlsson, J.L. Intracellular conjugation and detoxification of palmitic acid by Mycobacterium tuberculosis. Journal of Bacteriology, 1954. 67(4). 456-459.

197. Hedgecock, L.W. Complexing of fatty acids by Triton WR1339 in relation to growth of Mycobacterium tuberculosis. Journal of Bacteriology, 1970. 103(2). 520-522.

198. McCarthy, C. Utilization of palmitic acid by Mycobacterium avium. Infection and Immunity, 1971. 4(3). 199-204.

199. McCarthy, C. Effect of palmitic acid utilization on cell division in Mycobacterium avium. Infection and Immunity, 1974. 9(2). 363-372.

200. Stavri, M., Schneider, R., O'Donnell, G. et al. The antimycobacterial components of hops (Humulus lupulus) and their dereplication. Phytotherapy Research, 2004. 18(9). 774-776.

201. Saravanakumar, D.E.M., Folb, P.I., Campbell, B.W. et al. Antimycobacterial Activity of the Red Alga Polysiphonia virgata. Pharmaceutical Biology, 2008. 46(4). 254-260.

202. Blunt, J.W., Copp, B.R., Munro, M.H.G. et al. Marine natural products. Natural Product Reports, 2011. 28(2). 196-268.

203. Blunt, J.W., Copp, B.R., Keyzers, R.A. et al. Marine natural products. Natural Product Reports, 2012. 29(2). 144-222.

204. Shiomi, K., Igarashi, T., Yokota, H. et al. Isolation and structures of grammistins, peptide toxins from the skin secretion of the soapfish Grammistes sexlineatus. Toxicon, 2000. 38(1). 91-103.

205. Moore, K.S., Wehrli, S., Roder, H. et al. Squalamine: an aminosterol antibiotic from the shark. Proceedings of the National Academy of Sciences, 1993. 90(4). 1354-1358.

206. Rao, M.N., Shinnar, A.E., Noecker, L.A. et al. Aminosterols from the Dogfish Shark Squalus acanthias. Journal of Natural Products, 2000. 63(5). 631-635.

207. Sorensen, P.W., Fine, J.M., Dvornikovs, V. et al. Mixture of new sulfated steroids functions as a migratory pheromone in the sea lamprey. Nature Chemical Biology, 2005. 1(6). 324-328.

208. Ciereszko, L.S., Odense, P.H., and Schmidt, R.W. Chemistry of coelenterates. II. Occurrence of taurobetaine and creatine in gorgonians. Annals of the New York Academy of Sciences, 1960. 90(3). 920-922. 
209. Gabant, M., Martin, M., Moriou, C.1. et al. Axiphenylalaninium and Axityrosinium, Modified Amino Acids from the Mediterranean Marine Sponge Axinella polypoides. Journal of Natural Products, 2009. 72(10). 1875-1878.

210. MarinLit. 2013; Available from: http://www.chem.canterbury.ac.nz/marinlit/marinlit.shtml.

211. Huang, R., Peng, Y., Zhou, X. et al. A new taurine derivative from South China Sea marine sponge Axinella sp. Natural Product Research, 2012.

212. Wang, B., Jin Lee, K., Zhang, S. et al. 2-Palmitamidoethanesulfonic acid, a taurine derivative from the marine sponge Haliclona sp. Chemistry of Natural Compounds, 2009. 45(1). 137-138.

213. Wang, W., Lee, Y.M., Hong, J.K. et al. New Taurine Derivatives from a Starfish and a Sponge. Natural Sciences product, 2003. 9(4). 241-244.

214. Zhou, X., Xu, T., Wen, K. et al. New N-Acyl Taurine from the Sea Urchin Glyptocidaris crenularis. Bioscience, Biotechnology, and Biochemistry, 2010. 74(5). 1089-1091.

215. Emura, C., Higuchi, R., and Miyamoto, T. Irciniasulfonic acid B, a novel taurine conjugated fatty acid derivative from a Japanese marine sponge, Ircinia sp. Tetrahedron, 2006. 62(24). 5682-5685.

216. Kaya, K. Chemistry and biochemistry of taurolipids. Progress in Lipid Research, 1992. 31(1). 87-108.

217. Nohara-Uchida, K. and Kaya, K. Inhibitory effect of taurolipids on Clostridium perfringens sialidase. Journal of Biochemistry (Tokyo, Japan), 1988. 103(5). 840-842.

218. Rothe, B., Rothe, B., Roggentin, P. et al. The sialidase gene from Clostridium septicum: cloning, sequencing, expression in Escherichia coli and identification of conserved sequences in sialidases and other proteins. Molecular and General Genetics MGG, 1991. 226(1-2). 190-197.

219. Ishiyama, H., Ishibashi, M., Ogawa, A. et al. Taurospongin A, a Novel Acetylenic Fatty Acid Derivative Inhibiting DNA Polymerase $\beta$ and HIV Reverse Transcriptase from Sponge Hippospongia sp. The Journal of Organic Chemistry, 1997. 62(12). 3831-3836.

220. Starcevic, D., Dalal, S., and Sweasy, J.B. Is there a link between DNA polymerase $\beta$ and cancer? Cell Cycle, 2004. 3(8). 998-1001.

221. McCulloch, M.W.B., Bugni, T.S., Concepcion, G.P. et al. Carteriosulfonic Acids $A-C$, GSK-3 $\beta$ Inhibitors from a Carteriospongia sp. Journal of Natural Products, 2009. 72(9). 1651-1656.

222. Logan, C.Y. and Nusse, R. The Wnt signaling pathway in development and disease. Annual Review of Cell and Developmental Biology, 2004. 20. 781-810.

223. Chou, T., Kuramoto, M., Otani, Y. et al. Pinnaic acid and tauropinnaic acid: Two novel fatty acids composing a 6-azaspiro[4.5]decane unit from the Okinawan bivalve Pinna muricata. Tetrahedron Letters, 1996. 37(22). 38713874.

224. Kobayashi, J., Inaba, K., and Tsuda, M. Tauroacidins A and B, new bromopyrrole alkaloids possessing a taurine residue from Hymeniacidon sponge. Tetrahedron, 1997. 53(49). 16679-16682.

225. Normanno, N., Maiello, M.R., and De Luca, A. Epidermal growth factor receptor tyrosine kinase inhibitors (EGFR-TKIs): simple drugs with a complex mechanism of action? Journal of Cellular Physiology, 2003. 194(1). 13-19. 
226. Jiménez, C. and Crews, P. Mauritamide A and accompanying oroidin alkaloids from the sponge Agelas mauritiana. Tetrahedron Letters, 1994. 35(9). 13751378.

227. Hertiani, T., Edrada-Ebel, R., Ortlepp, S. et al. From anti-fouling to biofilm inhibition: New cytotoxic secondary metabolites from two Indonesian Agelas sponges. Bioorganic \& Medicinal Chemistry, 2010. 18(3). 1297-1311.

228. Fattorusso, E. and Taglialatela-Scafati, O. Two novel pyrrole-imidazole alkaloids from the Mediterranean sponge Agelas oroides. Tetrahedron Letters, 2000. 41(50). 9917-9922.

229. Endo, T., Tsuda, M., Okada, T. et al. Nagelamides A-H, new dimeric bromopyrrole alkaloids from marine sponge Agelas species. Journal of Natural Products, 2004. 67(8). 1262-1267.

230. Wright, A., Nielson, J., Tapiolas, D. et al. Detailed NMR, Including 1,1ADEQUATE, and Anticancer Studies of Compounds from the Echinoderm Colobometra perspinosa. Marine Drugs, 2009. 7(4). 565-575.

231. Cafieri, F., Fattorusso, E., and Taglialatela-Scafati, O. Novel betaines from the marine sponge Agelas dispar. Journal of Natural Products, 1998. 61(9). 11711173.

232. Concepcion, G.P., Foderaro, T.A., Eldredge, G.S. et al. Topoisomerase IIMediated DNA Cleavage by Adocia- and Xestoquinones from the Philippine Sponge Xestospongia sp. Journal of Medicinal Chemistry, 1995. 38(22). 45034507.

233. Hande, K.R. Topoisomerase II inhibitors. Update on Cancer Therapeutics, 2008. 3(1). 13-26.

234. Sandoval, I.T., Davis, R.A., Bugni, T.S. et al. Cytotoxic isoquinoline quinones from sponges of the genus Petrosia. Natural Product Research, 2004. 18(1). 8993.

235. Takahashi, Y., Kubota, T., Shibazaki, A. et al. Nakijinamines $C-E$, New Heteroaromatic Alkaloids from the Sponge Suberites Species. Organic Letters, 2011. 13(12). 3016-3019.

236. Killday, K.B., Yarwood, D., Sills, M.A. et al. Microxine, a New cdc2 Kinase Inhibitor from the Australian Marine Sponge Microxina Species. Journal of Natural Products, 2001. 64(4). 525-526.

237. Malumbres, M., Pevarello, P., Barbacid, M. et al. CDK inhibitors in cancer therapy: what is next? Trends in pharmacological sciences, 2008. 29(1). 16-21.

238. Hosoya, T., Hirokawa, T., Takagi, M. et al. Trichostatin Analogues JBIR-109, JBIR-110, and JBIR-111 from the Marine Sponge-Derived Streptomyces sp. RM72. Journal of Natural Products, 2012. 75(2). 285-289.

239. Wagner, J.M., Hackanson, B., Lubbert, M. et al. Histone deacetylase (HDAC) inhibitors in recent clinical trials for cancer therapy. Clinical Epigenetics., 2010. 1(3-4). 117-136.

240. He, H.Y., Faulkner, D.J., Lee, A.Y. et al. A new imidazole alkaloid from the marine sponge Leucetta microrhaphis. The Journal of Organic Chemistry, 1992. 57(7). 2176-2178.

241. Stochaj, W.R., Dunlap, W.C., and Shick, J.M. Two new UV-absorbing mycosporine-like amino acids from the sea anemone Anthopleura elegantissima and the effects of zooxanthellae and spectral irradiance on chemical composition and content. Marine Biology, 1994. 118(1). 149-156. 
242. Teruya, T., Suenaga, K., Maruyama, S. et al. Biselides A-E: novel polyketides from the Okinawan ascidian Didemnidae sp. Tetrahedron, 2005. 61(27). 65616567.

243. Huang, L.-S., He, F., Huang, H. et al. Carbamate derivatives and sesquiterpenoids from the South China Sea gorgonian Melitodes squamata. Beilstein Journal of Organic Chemistry, 2012. 8. 170-176.

244. Gupta, P., Sharma, U., Schulz, T.C. et al. Bicyclic C21 Terpenoids from the Marine Sponge Clathria compressa. Journal of Natural Products, 2012. 75(6). 1223-1227.

245. De Marino, S., Iorizzi, M., Zollo, F. et al. New pyridinium alkaloids from a marine sponge of the genus Spongia with a human phospholipase A(2) inhibitor profile. Journal of Natural Products, 2000. 63(3). 322-326.

246. Murakami, M. and Kudo, I. Phospholipase A2. Journal of Biochemistry (Tokyo, Japan), 2002. 131(3). 285-292.

247. Zhang, H. and Capon, R.J. Phorbasins D-F: Diterpenyl-taurines from a Southern Australian Marine Sponge, Phorbas sp. Organic Letters, 2008. 10(10). 1959-1962.

248. Jeon, J.E., Liao, L., Kim, H. et al. Cytotoxic Diterpenoid Pseudodimers from the Korean Sponge Phorbas gukhulensis. Journal of Natural Products, 2013. 76(9). 1679-1685.

249. Aiello, A., Fattorusso, E., Luciano, P. et al. Isolation and Structure Determination of Aplidinones A-C from the Mediterranean Ascidian Aplidium conicum: A Successful Regiochemistry Assignment by Quantum Mechanical 13C NMR Chemical Shift Calculations. European Journal of Organic Chemistry, 2005. 2005(23). 5024-5030.

250. Grube, A., Assmann, M., Lichte, E. et al. Bioactive metabolites from the Caribbean sponge Aka coralliphagum. Journal of Natural Products, 2007. 70(4). 504-509.

251. Takahashi, Y., Ushio, M., Kubota, T. et al. Nakijiquinones $J-R$, Sesquiterpenoid Quinones with an Amine Residue from Okinawan Marine Sponges $\%$. Journal of Natural Products, 2009. 73(3). 467-471.

252. Alvi, K.A., Diaz, M.C., Crews, P. et al. Evaluation of new sesquiterpene quinones from two Dysidea sponge species as inhibitors of protein tyrosine kinase. The Journal of Organic Chemistry, 1992. 57(24). 6604-6607.

253. Levitzki, A. Tyrosine kinase inhibitors: views of selectivity, sensitivity, and clinical performance. Annual Review of Pharmacology and Toxicology, 2013. 53. 161-185.

254. Giannini, C., Debitus, C., Lucas, R. et al. New Sesquiterpene Derivatives from the Sponge Dysidea Species with a Selective Inhibitor Profile against Human Phospholipase A2 and Other Leukocyte Functions. Journal of Natural Products, 2001. 64(5). 612-615.

255. Finamore, E., Minale, L., Riccio, R. et al. Novel marine polyhydroxylated steroids from the starfish Myxoderma platyacanthum. The Journal of Organic Chemistry, 1991. 56(3). 1146-1153.

256. Levina, E.V., Kalinovsky, A.I., Andriyashchenko, P.V. et al. Trofosides A and B and other cytostatic steroid-derived compounds from the far east starfish Trofodiscus über. Russian Journal of Bioorganic Chemistry, 2007. 33(3). 334340 . 
257. Chludil, H.D. and Maier, M.S. Minutosides A and B, antifungal sulfated steroid xylosides from the patagonian starfish Anasterias minuta. Journal of Natural Products, 2005. 68(8). 1279-1283.

258. Tang, H.F., Cheng, G., Wu, J. et al. Cytotoxic Asterosaponins Capable of Promoting Polymerization of Tubulin from the Starfish Culcita novaeguineae. Journal of Natural Products, 2009. 72(2). 284-289.

259. Keyzers, R.A., Gray, C.A., Schleyer, M.H. et al. Malonganenones A-C, novel tetraprenylated alkaloids from the Mozambique gorgonian Leptogorgia gilchristi. Tetrahedron, 2006. 62(10). 2200-2206.

260. Gu, S.-X., Li, Z.-M., Ma, X.-D. et al. Chiral resolution, absolute configuration assignment and biological activity of racemic diarylpyrimidine $\mathrm{CH}(\mathrm{OH})-\mathrm{DAPY}$ as potent nonnucleoside HIV-1 reverse transcriptase inhibitors. European Journal of Medicinal Chemistry, 2012. 53(0). 229-234.

261. Edrada, R.A., Heubes, M., Brauers, G. et al. Online Analysis of Xestodecalactones A-C, Novel Bioactive Metabolites from the Fungus Penicillium cf. montanense and Their Subsequent Isolation from the Sponge Xestospongia exigua\#. Journal of Natural Products, 2002. 65(11). 1598-1604.

262. Dewick, P.M. The biosynthesis of C5-C25 terpenoid compounds. Natural Product Reports, 2002. 19(2). 181-222.

263. Fürstner, A., De Souza, D., Turet, L. et al. Total Syntheses of the Actin-Binding Macrolides Latrunculin A, B, C, M, S and 16-epi-Latrunculin B. Chemistry - A European Journal, 2007. 13(1). 115-134.

264. Antunes, E.M., Copp, B.R., Davies-Coleman, M.T. et al. Pyrroloiminoquinone and related metabolites from marine sponges. Natural Product Reports, 2005. 22(1). 62-72.

265. Spector, I., Shochet, N.R., Kashman, Y. et al. Latrunculins: Novel Marine Toxins that Disrupt Microfilament Organization in Cultured Cells. Science, 1983. 219(4584). 493-495.

266. Coue, M., Brenner, S.L., Spector, I. et al. Inhibition of actin polymerization by latrunculin A. FEBS Letters, 1987. 213(2). 316-318.

267. Spector, I., Shochet, N.R., Blasberger, D. et al. Latrunculins-novel marine macrolides that disrupt microfilament organization and affect cell growth: I. Comparison with cytochalasin D. Cell Motility and the Cytoskeleton, 1989. 13(3). 127-144.

268. Capon, R.J., MacLeod, J.K., and Willis, A.C. Trunculins A and B, norsesterterpene cyclic peroxides from a marine sponge, Latrunculia brevis. The Journal of Organic Chemistry, 1987. 52(3). 339-342.

269. Hu, J.F., Fan, H., Xiong, J. et al. Discorhabdins and pyrroloiminoquinonerelated alkaloids. Chemical Reviews (Washington, DC, United States), 2011. 111(9). 5465-5491.

270. Perry, N.B., Blunt, J.W., McCombs, J.D. et al. Discorhabdin C, a highly cytotoxic pigment from a sponge of the genus Latrunculia. The Journal of Organic Chemistry, 1986. 51(26). 5476-5478.

271. Sepe, V., D'Orsi, R., Borbone, N. et al. Callipeltins F-I: new antifungal peptides from the marine sponge Latrunculia sp. Tetrahedron, 2006. 62(5). 833-840.

272. Muller, P.Y. and Milton, M.N. The determination and interpretation of the therapeutic index in drug development. Nature Reviews Drug Discovery, 2012. 11(10). 751-761. 
273. Kossuga, M.H., MacMillan, J.B., Rogers, E.W. et al. (2S,3R)-2-aminododecan3-ol, a new antifungal agent from the ascidian Clavelina oblonga. Journal of Natural Products, 2004. 67(11). 1879-1881.

274. Sata, N.U. and Fusetani, N. Amaminols A and B, new bicyclic amino alcohols from an unidentified tunicate of the family Polyclinidae. Tetrahedron Letters, 2000. 41(4). 489-492.

275. Aiello, A., Fattorusso, E., Giordano, A. et al. Clavaminols A-F, novel cytotoxic 2-amino-3-alkanols from the ascidian Clavelina phlegraea. Bioorganic \& Medicinal Chemistry Letters, 2007. 15(8). 2920-2926.

276. Ciavatta, M.L., Manzo, E., Nuzzo, G. et al. Crucigasterins A-E, antimicrobial amino alcohols from the Mediterranean colonial ascidian Pseudodistoma crucigaster. Tetrahedron, 2010. 66(38). 7533-7538.

277. Jares-Erijman, E.A., Bapat, C.P., Lithgow-Bertelloni, A. et al. Crucigasterins, new polyunsaturated amino alcohols from the mediterranean tunicate Pseudodistoma crucigaster. Journal of Organic Chemistry, 1993. 58(21). 57325737.

278. Garrido, L., Zubía, E., Ortega, M.a.J. et al. Obscuraminols, new unsaturated amino alcohols from the tunicate Pseudodistoma obscurum: structure and absolute configuration. Tetrahedron, 2001. 57(21). 4579-4588.

279. Perry, N., Blunt, J., and Munro, M. Cytotoxic and Antifungal $C_{14}$ Amines From a New Zealand Ascidian: Major Lipid Components of Pseudodistoma novaezelandiae. Australian Journal of Chemistry, 1991. 44(4). 627-633.

280. Ishibashi, M., Ohizumi, Y., Sasaki, T. et al. Pseudodistomins A and B, novel antineoplastic piperdine alkaloids with calmodulin antagonistic activity from the Okinawan tunicate Pseudodistoma kanoko. The Journal of Organic Chemistry, 1987. 52(3). 450-453.

281. Freyer, A.J., Patil, A.D., Killmer, L. et al. Three New Pseudodistomins, Piperidine Alkaloids from the Ascidian Pseudodistoma megalarva. Journal of Natural Products, 1997. 60(10). 986-990.

282. Hooper, G.J., Davies-Coleman, M.T., and Coetzee, P.S. New antimicrobials C14 and C13 amines from a South African marine ascidian. Natural Product Letters, 1995. 6(1). 31-35.

283. Gulavita, N.K. and Scheuer, P.J. Two epimeric aliphatic amino alcohols from a sponge. Xestospongia sp. Journal of Organic Chemistry, 1989. 54(2). 366-369.

284. Jiménez, C. and Crews, P. Novel Marine Sponge Amino Acids, 10. Xestoaminols from Xestaspongia sp. Journal of Natural Products, 1990. 53(4). 978-982.

285. Clark, R.J., Garson, M.J., and Hooper, J.N.A. Antifungal Alkyl Amino Alcohols from the Tropical Marine Sponge Haliclona n. sp. Journal of Natural Products, 2001. 64(12). 1568-1571.

286. Massard, C., Salazar, R., Armand, J.P. et al. Phase I dose-escalating study of ES-285 given as a three-hour intravenous infusion every three weeks in patients with advanced malignant solid tumors. Investigational New Drugs, 2012. 30(6). 2318-2326.

287. Vilar, E., Grünwald, V., Schöffski, P. et al. A phase I dose-escalating study of ES-285, a marine sphingolipid-derived compound, with repeat dose administration in patients with advanced solid tumors. Investigational New Drugs, 2012. 30(1). 299-305.

288. Schöffski, P., Dumez, H., Ruijter, R. et al. Spisulosine (ES-285) given as a weekly three-hour intravenous infusion: results of a phase I dose-escalating 
study in patients with advanced solid malignancies. Cancer Chemotherapy and Pharmacology, 2011. 68(6). 1397-1403.

289. Baird, R.D., Kitzen, J., Clarke, P.A. et al. Phase I safety, pharmacokinetic, and pharmacogenomic trial of ES-285, a novel marine cytotoxic agent, administered to adult patients with advanced solid tumors. Molecular Cancer Therapeutics, 2009. 8(6). 1430-1437.

290. Cuadros, R., Montejo de Garcini, E., Wandosell, F. et al. The marine compound spisulosine, an inhibitor of cell proliferation, promotes the disassembly of actin stress fibers. Cancer Letters, 2000. 152(1). 23-29.

291. Salcedo, M., Cuevas, C., Alonso, J.L. et al. The marine sphingolipid-derived compound ES 285 triggers an atypical cell death pathway. Apoptosis, 2007. 12(2). 395-409.

292. Sánchez, A.M., Malagarie-Cazenave, S., Olea, N. et al. Spisulosine (ES-285) induces prostate tumor PC-3 and LNCaP cell death by de novo synthesis of

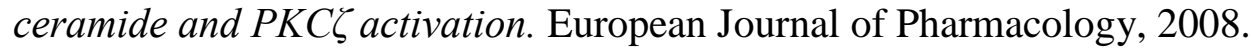
584(2-3). 237-245.

293. Zhang, P., Liu, B., Jenkins, G.M. et al. Expression of Neutral Sphingomyelinase Identifies a Distinct Pool of Sphingomyelin Involved in Apoptosis. Journal of Biological Chemistry, 1997. 272(15). 9609-9612.

294. Veldman, R.J., Klappe, K., Hoekstra, D. et al. Metabolism and apoptotic properties of elevated ceramide in HT29rev cells. Biochemical Journal, 1998. 331 ( Pt 2). 563-569.

295. Obeid, L.M., Linardic, C.M., Karolak, L.A. et al. Programmed cell death induced by ceramide. Science, 1993. 259(5102). 1769-1769.

296. Abad, J.L., Nieves, I., Rayo, P. et al. Straightforward Access to Spisulosine and 4,5-Dehydrospisulosine Stereoisomers: Probes for Profiling Ceramide Synthase Activities in Intact Cells. Journal of Organic Chemistry, 2013. 78(12). 58585866.

297. Ishibashi, M., Deki, K., and Kobayashi, J.i. Revised Structure of Pseudodistomin A, a Piperidine Alkaloid Isolated from the Okinawan Tunicate Pseudodistoma kanoko. Journal of Natural Products, 1995. 58(5). 804-806.

298. Kong, F. and Faulkner, D.J. Leucettamines $A$ and B, two antimicrobial lipids from the calcareous sponge Leucetta microraphis. The Journal of Organic Chemistry, 1993. 58(4). 970-971.

299. Tsukamoto, S., Takeuchi, T., Rotinsulu, H. et al. Leucettamol A: A new inhibitor of Ubc13-Uev1A interaction isolated from a marine sponge, Leucetta aff. microrhaphis. Bioorganic \& Medicinal Chemistry Letters, 2008. 18(24). 63196320.

300. Willis, R.H. and De Vries, D.J. BRS1, A C30 BIS-Amino, BIS-Hydroxy Polyunsaturated lipid from an Australian calcareous sponge that inhibits protein kinase $C$. Toxicon, 1997. 35(7). 1125-1129.

301. Makarieva, T.N., Guzii, A.G., Denisenko, V.A. et al. New two-headed sphingolipid-like compounds from the marine sponge Oceanapia sp. Russian Chemical Bulletin, 2008. 57(3). 669-673.

302. Makarieva, T.N., Denisenko, V.A., Stonik, V.A. et al. Rhizochalin, a novel secondary metabolite of mixed biosynthesis from the sponge Rhizochalina incrustata. Tetrahedron Letters, 1989. 30(47). 6581-6584.

303. Makarieva, T.N., Zakharenko, A.M., Denisenko, V.A. et al. Rhizochalinin A, a new antileukemic two-headed sphingolipid from the sponge Rhizochalina incrustata. Chemistry of Natural Compounds, 2007. 43(4). 468-469. 
304. Makarieva, T.N., Dmitrenok, P.S., Zakharenko, A.M. et al. Rhizochalins $C$ and $D$ from the Sponge Rhizochalina incrustata. A Rare threo-Sphingolipid and a Facile Method for Determination of the Carbonyl Position in $\alpha, \omega$ -

Bifunctionalized Ketosphingolipids. Journal of Natural Products, 2007. 70(12). 1991-1998.

305. Makarieva, T., Zakharenko, A., Dmitrenok, P. et al. Isorhizochalin: a Minor Unprecedented Bipolar Sphingolipid of Stereodivergent Biogenesis from the Rhizochalina incrustata. Lipids, 2009. 44(12). 1155-1162.

306. Nicholas, G.M., Hong, T.W., Molinski, T.F. et al. Oceanapiside, an Antifungal Bis- $\alpha, \omega$-amino Alcohol Glycoside from the Marine Sponge Oceanapia phillipensis. Journal of Natural Products, 1999. 62(12). 1678-1681.

307. Zhou, B.N., Mattern, M.P., Johnson, R.K. et al. Structure and stereochemistry of a novel bioactive sphingolipid from a Calyx sp. Tetrahedron, 2001. 57(47). 9549-9554.

308. Perez, E., Gavigan, J.A., Otal, I. et al. Tn611 transposon mutagenesis in Mycobacterium smegmatis using a temperature-sensitive delivery system. Methods in Molecular Biology, 1998. 101. 187-198.

309. Dale, J.A. and Mosher, H.S. Nuclear magnetic resonance nonequivalence of diastereomeric esters of $\alpha$-substituted phenylacetic acids for the determination of stereochemical purity. Journal of the American Chemical Society, 1968. 90(14). 3732-3738.

310. Dale, J.A., Dull, D.L., and Mosher, H.S. $\alpha$-Methoxy- $\alpha$ trifluoromethylphenylacetic acid, a versatile reagent for the determination of enantiomeric composition of alcohols and amines. The Journal of Organic Chemistry, 1969. 34(9). 2543-2549.

311. Seco, J.M., Quiñoá, E., and Riguera, R. The Assignment of Absolute Configuration by $N M R \dagger$. Chemical Reviews (Washington, DC, United States), 2004. 104(1). 17-118.

312. De Silva, E.D., Geiermann, A.S., Mitova, M.I. et al. Isolation of 2-Pyridone Alkaloids from a New Zealand Marine-Derived Penicillium species. Journal of Natural Products, 2009. 72(3). 477-479.

313. Cardellina, J.H. and Moore, R.E. Sphingosine derivatives from red algae of the ceramiales. Phytochemistry, 1978. 17(3). 554-555.

314. Garg, H.S., Sharma, M., Bhakuni, D.S. et al. An antiviral sphingosine derivative from the green alga Ulva fasciata. Tetrahedron Letters, 1992. 33(12). 16411644.

315. Winzeler, E.A., Shoemaker, D.D., Astromoff, A. et al. Functional characterization of the $S$. cerevisiae genome by gene deletion and parallel analysis. Science, 1999. 285(5429). 901-906.

316. Worgall, T.S. Sphingolipids: major regulators of lipid metabolism. Current opinion in clinical nutrition and metabolic care., 2007. 10(2). 149-155. 